\title{
Preliminary Aerodynamic Design of a Supercritical Carbon Dioxide Centrifugal Compressor
}

by

Alberto José Martel Matos

A thesis submitted to the Faculty of Graduate and Postdoctoral Affairs in partial fulfilments of the requirements for the degree of

Master of Applied Science

In

Mechanical Engineering

Department of Mechanical and Aerospace Engineering

Carleton University,

Ottawa, Ontario, Canada

December 2017

Copyright (C) 2017 by Alberto José Martel Matos 


\begin{abstract}
The preliminary sizing and design of a centrifugal compressor for a $10 \mathrm{MW}_{\mathrm{e}}$ waste heat recovery supercritical carbon dioxide $\left(\mathrm{S}-\mathrm{CO}_{2}\right)$ Brayton power plant is presented. The 3D aerodynamic analysis of the impeller and diffuser was completed using ANSYS CFX V17.2. Modelling and achieving proper convergence at the design point is challenging due to the proximity to the critical point. To overcome this challenge dimensional analysis was used.

Three independent non-dimensional parameters were used to perform the scaling between the design point and a partially dynamically and geometrically similar operating point, which lies in the supercritical ideal gas region of carbon dioxide. The independent parameters describe compressible non-ideal flow by incorporating the isentropic exponent $\left(\mathrm{n}_{\mathrm{s}}\right)$ and compressibility factor $(\mathrm{Z})$.

The investigation found that the compressor characteristic map can be expressed as a single map referred to as the Generalized Map. The map is expressed in terms of the Enthalpy Rise coefficient $\left(\Pi_{\mathrm{h}}\right)$ and the Flow Coefficient $\left(\Pi_{\mathrm{m}}\right)$ for a set of constant speed lines. From the Generalized Map, the designer can project the characteristic map corresponding to another operating point. The projection methodology is valid within the region where the isentropic exponent $\left(\mathrm{n}_{\mathrm{s}}\right)$ remains constant. The accuracy of the projection methodology in the region where the isentropic exponent varies is unknown; further investigation is recommended to fully understand the effects of the isentropic exponent in these regions.
\end{abstract}




\section{Acknowledgments}

I would like to express my greatest gratitude towards my two supervisors, professor Dr. Henry Saari, and professor Dr. Steen A. Sjolander, for their invaluable guidance and support throughout this journey. I would also like to thank Dr. Henry Saari and Doosan Heavy Industries \& Construction Co., Ltd. for the financial support and the opportunity to take part of this great project.

I would also like to thank my mother Magaly, my father Alberto and my brother André for their unconditional support. My parents have sacrificed so much to always provide the best for me. Throughout this journey my parents have always been supportive and pushed me to do better.

I would also like to thank my close friends and CUKC for always being there. I appreciate the tips and guidance provided by my friend Amr Daouk. I would like to thank Turner Strang for all the discussions we had regarding CFD and this project. I would also like to express my gratitude to my friend Rebecca Robinson for taking the time to provide feedback regarding my writing. 


\section{Table of Contents}

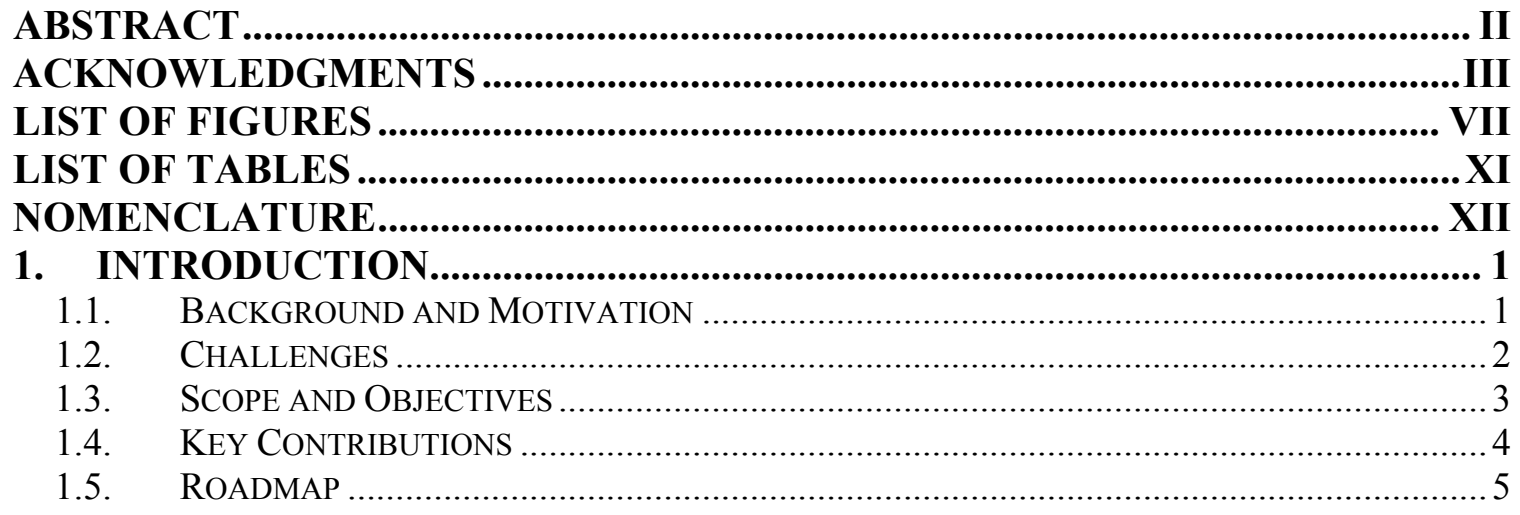

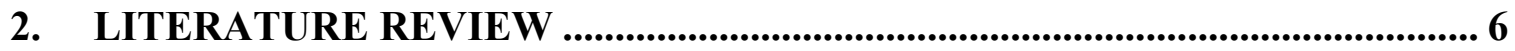

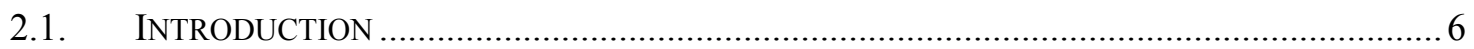

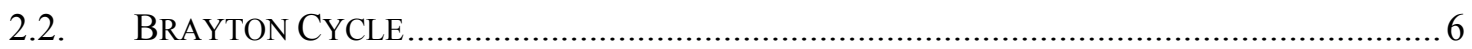

2.3. BENEFITS OF SUPERCRITICAL CARBON DIOXIDE BRAYTON POWER CYCLES................. 7

2.3.1. Expected Efficiency and Temperature Operating Range.......................... 7

2.3.2. Size of Components ....................................................................... 8

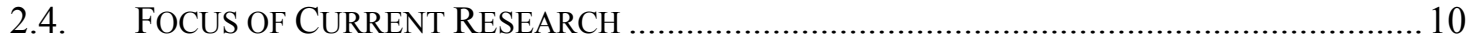

2.5. MOTIVATION FOR THE DESIGN OF A $10 \mathrm{MW}_{\mathrm{E}}$ SYSTEM............................................... 10

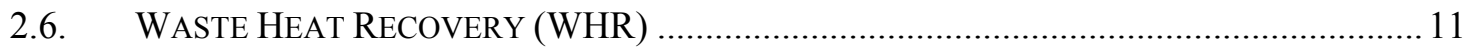

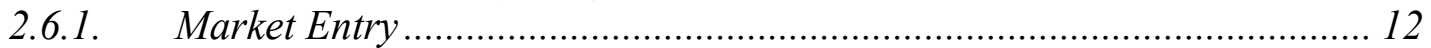

2.7. GAS TURBINE DESIGN PROCESS -PRELIMINARY DESIGN ………............................... 13

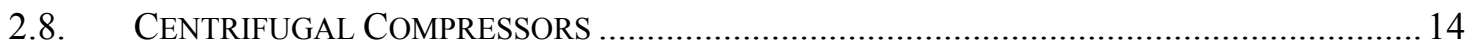

2.9. INITIAL SIZING OF CENTRIFUGAL COMPRESSORS ………………….......................... 16

2.9.1. De Haller Number....................................................................... 17

2.9.2. Flow Coefficient ( $\varphi 2)$ - Limits of the Surge and Choke Region .............. 18

2.9.3. Mass Flow Surge Margin.................................................................. 19

2.9.4. Vaneless Diffuser: Sizing and Pressure losses ....................................... 20

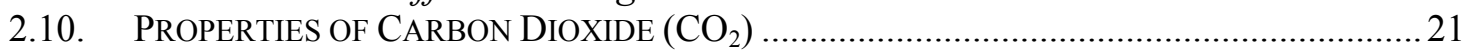

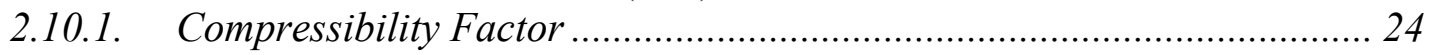

2.10.2. Properties of S-CO $\mathrm{CO}_{2}$ near the Critical Point ......................................... 28

2.10.3. Isentropic Exponent ………………………................................ 33

2.10.4. Speed of Sound .............................................................................. 35

2.10.5. Dimensional Analysis and Non-Dimensional Parameters for Non-Ideal

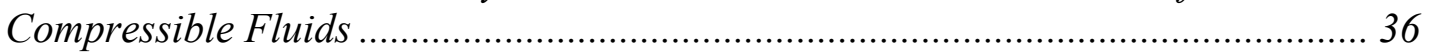

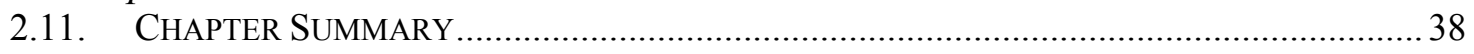

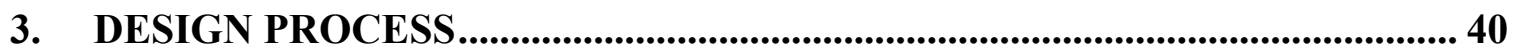

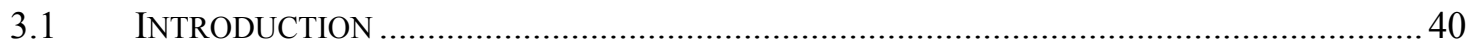

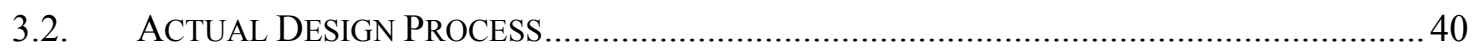

3.3. DESIGN POINT \#1 VERSUS DESIGN POINT \#2 …………............................................. 44

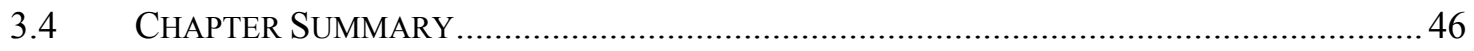

4. DESIGN POINT, INITIAL SIZING AND SCALING METHODOLOGY...... 48

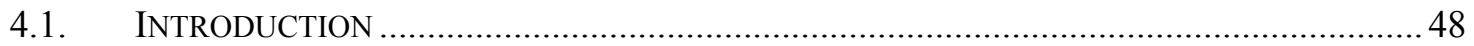

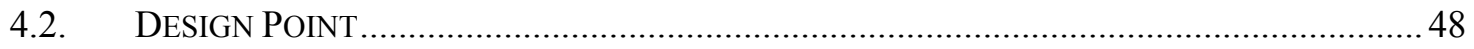

4.2.1. Two-phase Region and Condensation .................................................. 51 


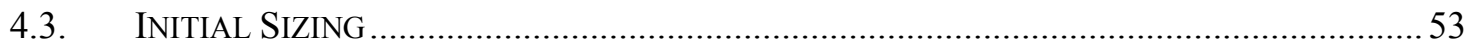

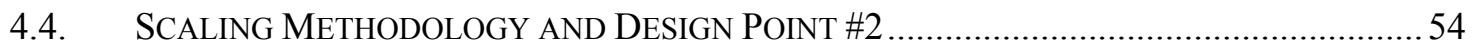

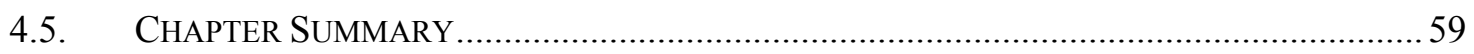

5. COMPUTATIONAL FLUID DYNAMICS FOR A SUPERCRITICAL CARBON DIOXIDE CENTRIFUGAL COMPRESSOR ............................................. 60

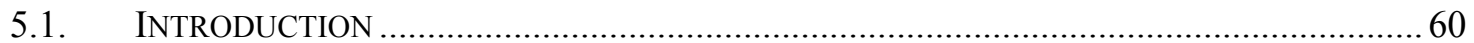

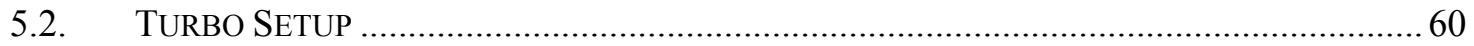

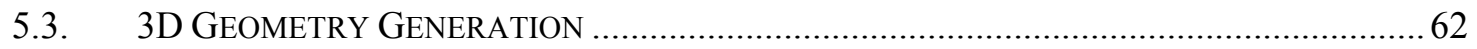

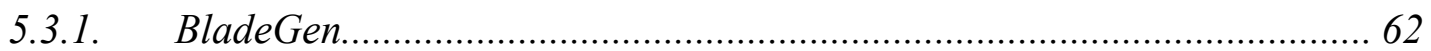

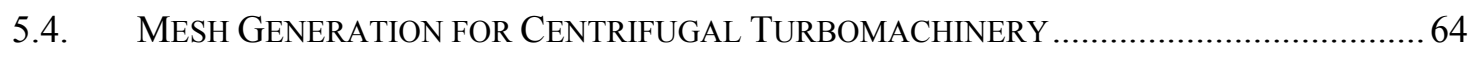

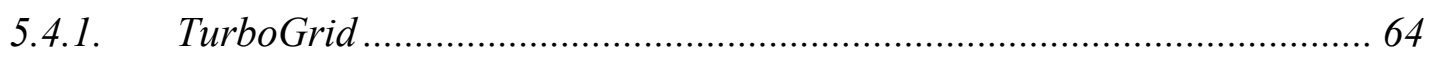

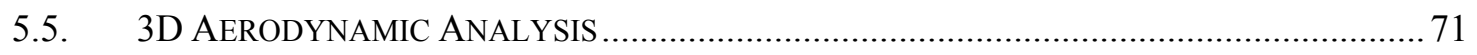

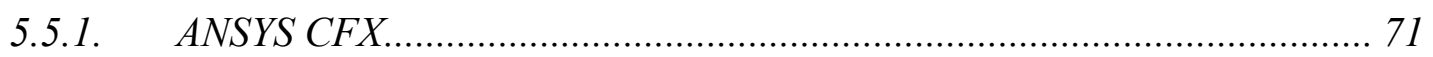

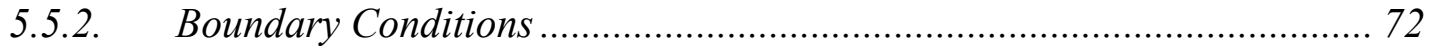

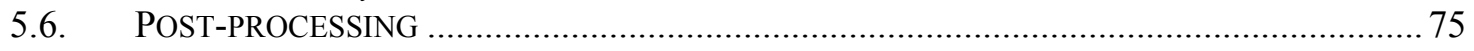

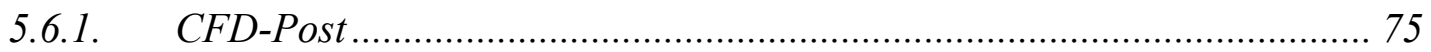

6. ANALYSIS AND DISCUSSION OF THE 3D AERODYNAMIC RESULT .... 76

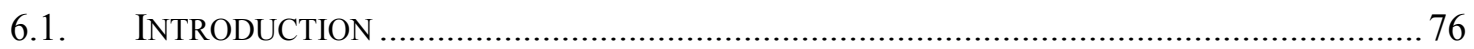

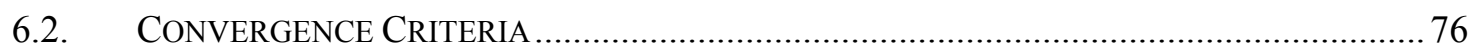

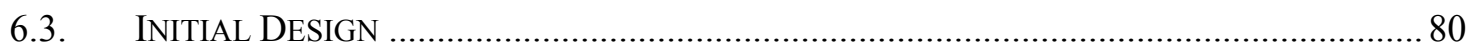

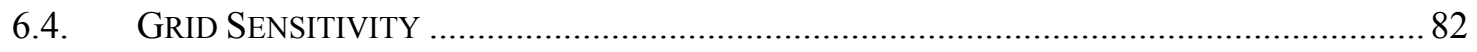

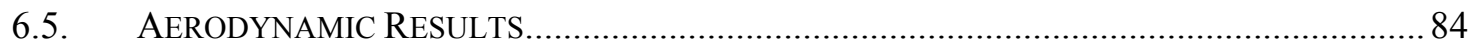

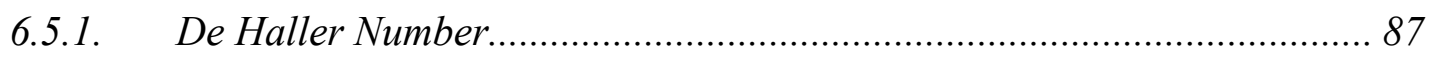

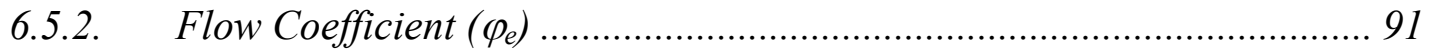

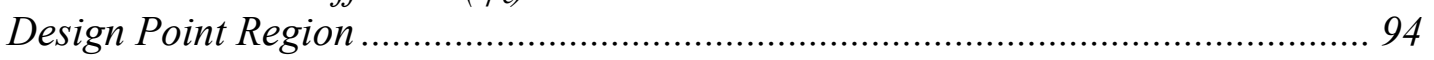

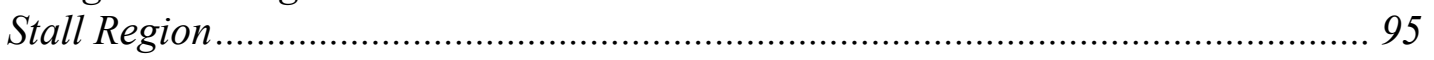

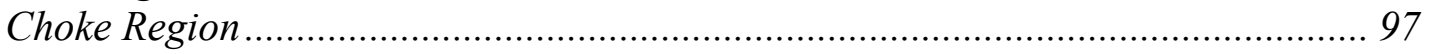

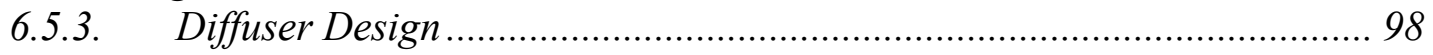

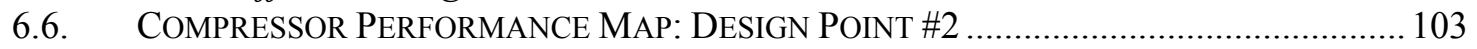

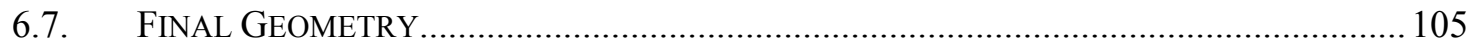

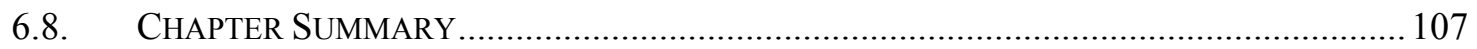

7. COMPRESSOR PERFORMANCE MAPS AND PROJECTION

METHODOLOGY …....................................................................................................... 109

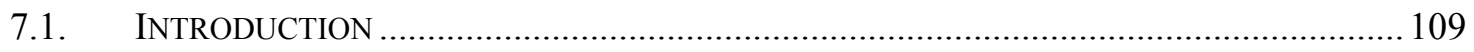

7.2. Dimensional ANALYSIS: DEPENDENT PARAMETERS FOR COMPRESSIBLE NON-IDEAL FLOWS 109

7.2.1. Enthalpy Rise Coefficient ............................................................... 110

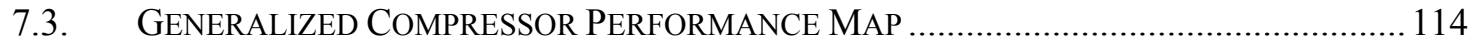

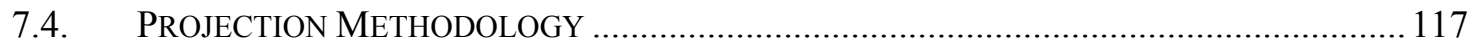

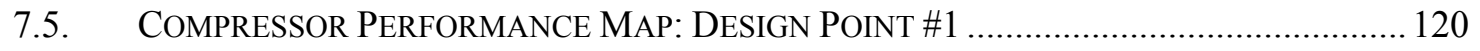

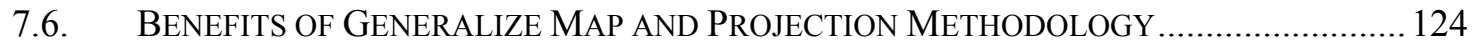

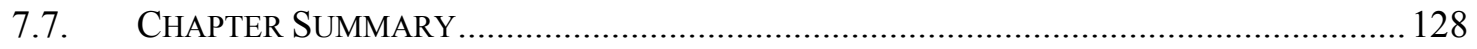

8. CONCLUSION AND RECOMMENDATIONS ........................................... 130

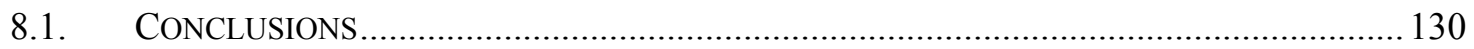

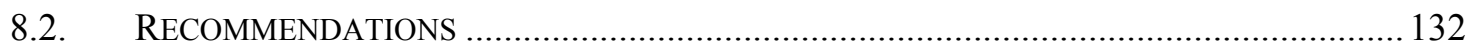

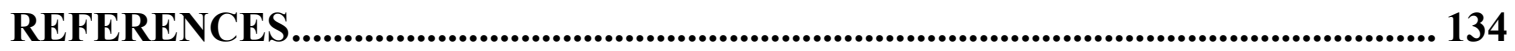


APPENDIX A: LIST OF IDENTIFIED WASTE HEAT SOURCES ...................... 138 APPENDIX B: INITIAL SIZING USING THE FLOW $(\phi)$ AND WORK $(\psi)$ COEFFICIENT .......................................................................................................... 139 


\section{List of Figures}

Figure 1-1:Diagram of a Simple Regenerative Brayton Cycle......................................... 1

Figure 2-1: Cycle Efficiencies vs Source Temperature for fixed component efficiency (SANDIA, 2013) .......................................................................................

Figure 2-2: Comparison of turbine sizes for power cycles using different fluids (Dostal, 2004).

\section{9}

Figure 2-3: Comparison of the size of heat exchangers between $\mathrm{S}-\mathrm{CO}_{2}$ and steam (Musgrove, 2016)....................................................................................... 9

Figure 2-4: Component and technology options for $\mathrm{S}_{-} \mathrm{CO}_{2}$ (SANDIA, 2013) ................. 11

Figure 2-5: Gas Turbine Design Process (Saravanamuttoo, 2009).................................. 13

Figure 2-6: Centrifugal compressor (Sjolander, 2015) ……………………............. 15

Figure 2-7:Total Enthalpy vs Entropy diagram $\left(\mathrm{h}_{0}-\mathrm{s}\right)$. Illustrates the ideal and actual compression processes that provide the same pressure ratio $\left(\mathrm{P}_{\mathrm{o} 2} / \mathrm{P}_{01}\right)$ (Sjolander,

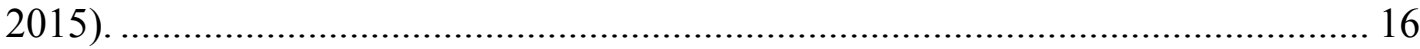

Figure 2-8: Variation of Slip Factor $(\mu)$ vs Flow Coefficient $(\varphi)$ (Eckardt 1980)............ 18

Figure 2-9: Types of vaneless diffusers (a) conical diffuser (expanding) diffuser, (b) constant width diffuser................................................................................... 20

Figure 2-10: Different degrees of "pinched" vaneless diffusers (Aungier, 2000),........... 21

Figure 2-11: Phase diagram of $\mathrm{CO}_{2}$ (REFPROP, 2015) .............................................. 23

Figure 2-12:Temperature versus Entropy diagram of $\mathrm{CO}_{2}$ (REFPROP, 2015)................ 24

Figure 2-13: Generalize compressibility factor chart for different fluids (Moran and Shapiro, 2011) ........................................................................................... 25

Figure 2-14: Compressibility factor $(\mathrm{Z})$ of $\mathrm{S}-\mathrm{CO}_{2}$ for a temperature range of $280 \mathrm{~K}$ to

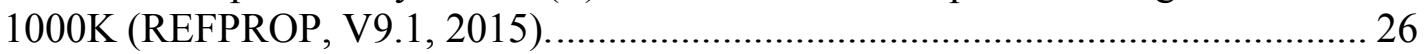

Figure 2-15: Compressibility factor $(\mathrm{Z})$ of $\mathrm{S}-\mathrm{CO}_{2}$ for a temperature range of $300 \mathrm{~K}$ to

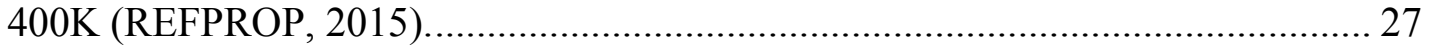

Figure 2-16: Description of the density values for different values of compressibility

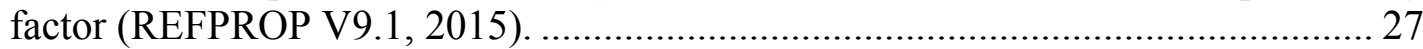

Figure 2-17: Plot of the constant specific heat $(\mathrm{Cp})$ near he critical pressure $(\mathrm{P}=7.39$ $\mathrm{MPa}$ ) and the corresponding pseudocritical points (REFPROP V9.1, 2015),......... 29

Figure 2-18: Plot of the constant specific heat $(\mathrm{Cp})$ at different isobars and the corresponding pseudocritical points (REFPROP V9.1, 2015).............................. 29

Figure 2-19: Density of $\mathrm{S}^{-\mathrm{CO}_{2}}$ for a temperature range of $280 \mathrm{~K}$ to $400 \mathrm{~K}$ (REFPROP

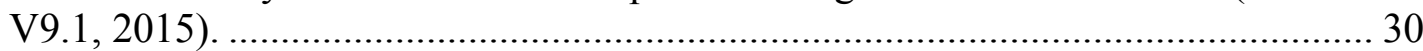

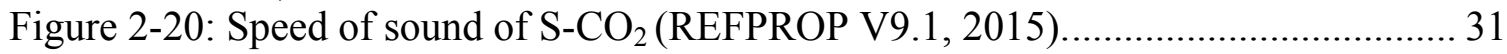

Figure 2-21: Speed of sound of $\mathrm{S}-\mathrm{CO}_{2}$ for a temperature range of $300 \mathrm{~K}$ to $380 \mathrm{~K}$

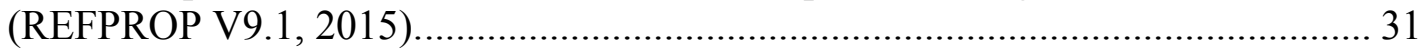

Figure 2-22: kinematic viscosity of S-CO $\mathrm{CO}_{2}(\mathrm{REFPROP}$ V9.1, 2015)............................ 32

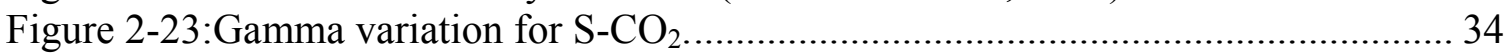

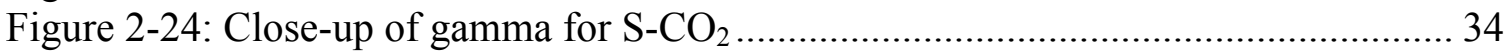

Figure 3-1: Common design process of a centrifugal compressor. .................................. 41

Figure 3-2: Modified design process, with the use of dimensional analysis and different Methodologies. Scaling methodology is highlighted in blue and the projection methodology is highlighted in green........................................................................ 43 
Figure 3-3: Compressibility factor of S- $\mathrm{CO}_{2}$. Orange circle highlights the area of real gas behaviour. Black circle indicates the location of Design Point \#1. The green ellipse highlights the area of ideal gas behavior. The red circle indicates the location of Design Point \#2. 44

Figure 3-4: Illustration of the Actual Design Point (Design Point \#1) and Modelling Design Point (Design Point \#2) which is the partially dynamically operating point.45

Figure 3-5: Design procedure and the content to each chapter in this thesis................... 47

Figure 4-1: Density of S-CO $\mathrm{CO}_{2}$ for a temperature range of $280 \mathrm{k}$ to $340 \mathrm{~K}$....................... 49

Figure 4-2: Initial dimensions of the centrifugal compressor. Note: curvature of the

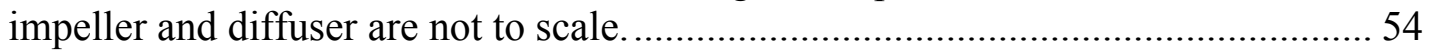

Figure 4-3: Steps required to implement the Scaling Methodology.............................. 57

Figure 5-1: Typical workflow of a centrifugal diffuser using Turbo Setup in ANSYS Workbench V17.2.

Figure 5-2: BladeGen graphical interface. (Top Left) illustrates the meridional view of the compressor and highlights the actual geometry of the inlet, impeller and diffuser. (Top Right) illustrates the actual geometry of the blades. (Bottom Left) provides the angles used to design the blades. (Bottom Right) allows the user to define the blade thickness. 63

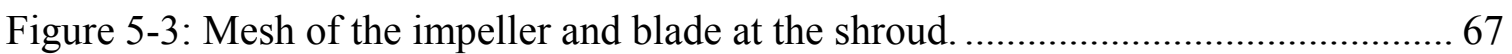

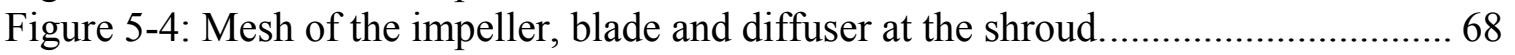

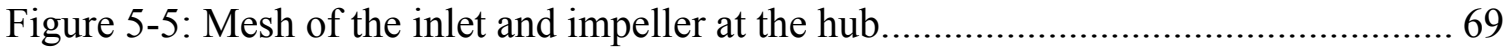

Figure 5-6: Mesh of the impeller, blade and diffuser at the hub.................................... 70

Figure 5-7: Mesh of the blade and impeller passage. ................................................. 71

Figure 5-8: Location of the Inlet and outlet boundary condition as well as the location of

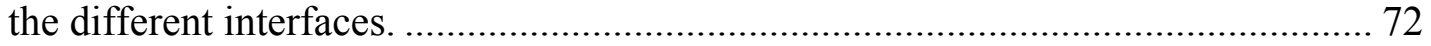

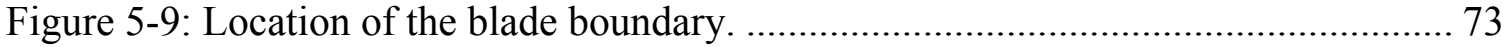

Figure 6-1: Constant speed line performance curve. The speed line corresponds to that of the design point rotational speed. Pressure ratio corresponds to total-to-total pressures. The Flow Coefficient $\left(\Pi_{\mathrm{e}}\right)$ is defined by Equation 2.32 ....................... 78

Figure 6-2: Illustrates the percent error $(\epsilon \mathrm{m})$ of the mass flow. The illustrated percent errors correspond to the design points of the constant speed line illustrated in Figure 6-1.

Figure 6-3: Constant speed line performance curve corresponding to the initial geometry (V2). The speed line corresponds to that of the design point. Pressure ratio corresponds to total-to-total pressures. The Flow Coefficient $\left(\Pi_{e}\right)$ is defined by Equation 2.32. 80

Figure 6-4: Illustrates the percent error $(\epsilon \mathrm{m})$ of the mass flow. The illustrated percent errors correspond to the design points of the constant speed line illustrated in Figure 6-3. 81

Figure 6-5: Summary of the grid sensitivity analysis. Figure illustrates the percent error corresponding to the mass flow between inlet and specified outlet....................... 83

Figure 6-6: Summary of the grid independence for pressure ratio and efficiency. ......... 83

Figure 6-7: Constant speed curve and efficiency curve of the design point rotational speed corresponding to the final preliminary geometry. ...................................... 85 
Figure 6-8: Summary of the De Haller Number for each of the different geometries investigated at the design point. The yellow region corresponds to the different min values of De Haller recommended by different authors. 88

Figure 6-9: Geometry investigation - estimate of the component's efficiency at the design point 88

Figure 6-10: Impeller outlet width (b) corresponding to each of the investigated geometries. 89

Figure 6-11: Blade-to-blade throat distance $(\mathrm{mm})$ corresponding to each of the investigated geometries............................................................................... 90

Figure 6-12: De Haller values corresponding to each operating point along the design

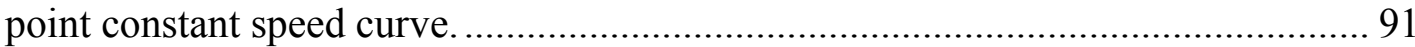

Figure 6-13: Constant speed curve and efficiency curve of the design point rotational speed corresponding to the final preliminary geometry. The red circle highlights the poor convergence near the stall region. ........................................................ 92

Figure 6-14: Design Point Region- Contour of relative velocity [m/s] at 10\% span. The

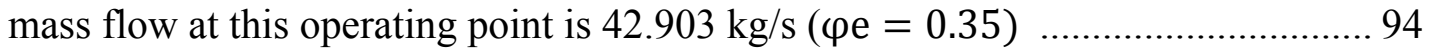

Figure 6-15: Design Point Region - Contour of relative velocity $[\mathrm{m} / \mathrm{s}]$ at $50 \%$ span. The

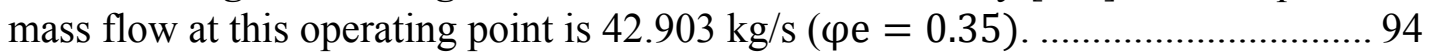

Figure 6-16: Design Point Region - Contour of relative velocity $[\mathrm{m} / \mathrm{s}]$ at $90 \%$ span. The

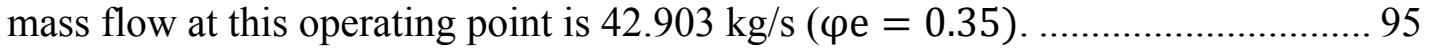

Figure 6-17: Stall Region - Contour of relative velocity [m/s] at $10 \%$ span. The mass

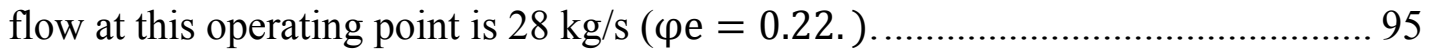

Figure 6-18: Stall Region - Contour of relative velocity [m/s] at 50\% span. The mass

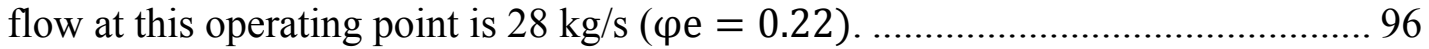

Figure 6-19: Stall Region - Contour of relative velocity [m/s] at $90 \%$ span. The mass

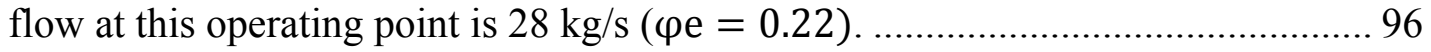

Figure 6-20: Choke Region - Contour of relative velocity [m/s] at $10 \%$ span. The mass

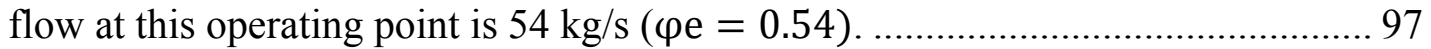

Figure 6-21: Choke Region - Contour of relative velocity [m/s] at 50\% span. The mass

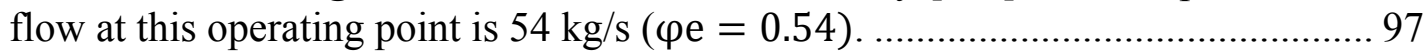

Figure 6-22: Choke Region - Contour of relative velocity [m/s] at $90 \%$ span. The mass

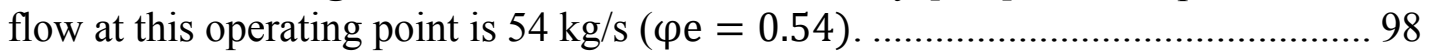

Figure 6-23: Summary of the recovery pressure (Cp) and the loss coefficient (K) corresponding to the design point at each of the investigated geometries.............. 99

Figure 6-24: Summary of the impeller pressure ratio and the impeller + diffuser pressure ratio corresponding to the design point at each of the investigated geometries. .... 100

Figure 6-25: Meridional velocity $[\mathrm{m} / \mathrm{s}$ ] inside the diffuser corresponding to the initial

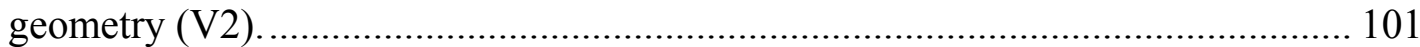

Figure 6-26: Summary of the pressure recovery $(\mathrm{Cp})$ and loss coefficient $(\mathrm{K})$ of the final geometry. Values correspond to the design point constant speed curve................. 102

Figure 6-27: Meridional velocity $[\mathrm{m} / \mathrm{s}]$ inside the diffuser corresponding to the final

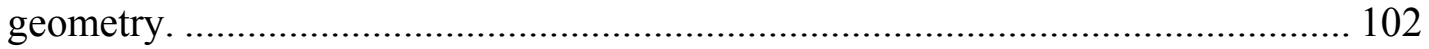

Figure 6-28: Compressor performance map corresponding to Design Point \#2 . .......... 104

Figure 6-29: Efficiency performance map corresponding to Design Point \#2 .............. 105

Figure 6-30: 3D geometry of the final preliminary impeller. (Left) Isometric view of the impeller. (Right) front view of impeller. ..................................................... 106 
Figure 6-31: 3D geometry of the diffuser. (Left) Isometric view of the diffuser. (Right) Cross-sectional view of the impeller 106

Figure 6-32: Final geometry sizing of the Impeller and diffuser. Note: curvature of impeller and diffuser not to scale. 107

Figure 7-1: Results of the validation study of a constant Enthalpy Rise for different values of Compressibility factor $(Z)$ and different fluids. 112

Figure 7-2: Generalized compressor performance map corresponding to the final preliminary geometry.......................................................................... 115

Figure 7-3: Efficiency performance map corresponding to Design Point \#2. It is important to understand that the efficiency corresponds to Design Point \#2. This value is the corrected to represent the estimated efficiency relative to the Reynolds Number of the dynamically similar operating point. 116

Figure 7-4: Compressor performance map corresponding to Design Point \#1 ............. 121

Figure 7-5: Compressor efficiency map corresponding to Design Point \#1................... 122

Figure 7-6: Value of isentropic exponent as a function of temperature for different isobars. The isentropic value corresponding to the pseudocritical line is illustrated by the black dash line. 123

Figure 7-7: Preliminary Performance Map for compressor inlet conditions of $\mathrm{P}_{\mathrm{o}, 1}=7.6$

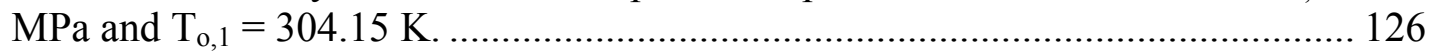

Figure 7-8: Preliminary Performance Map for compressor inlet conditions of Po, $1=8.4$

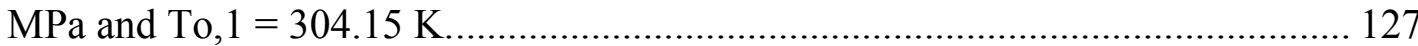

Figure 7-9: Preliminary Performance Map for compressor inlet conditions of Po, $1=9.0$

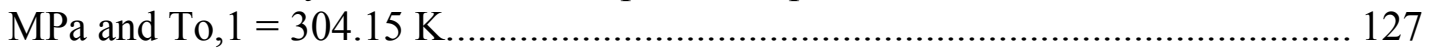

Figure B-1: Work Coefficient vs. Flow Coefficient covered impeller design targets (Sjolander, 2015) 140 


\section{List of Tables}

Table 2-1: Expected operating conditions for the $\mathrm{S}_{-} \mathrm{CO}_{2}$ power cycles (Adapted from

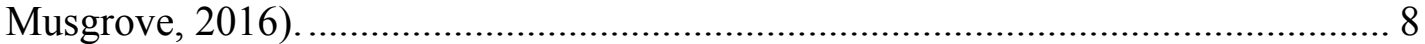

Table 2-2: Typical thermal efficiencies for power generation options, adapted from

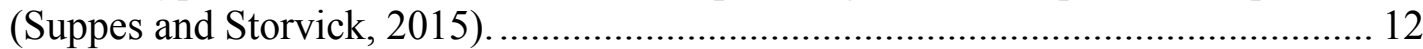

Table 2-3: Literature recommended De Haller values................................................ 17

Table 2-4: Summary of Critical temperature and pressure for different fluids (REFPROP,

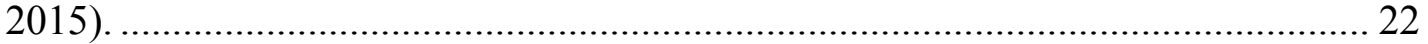

Table 4-1: Compressor inlet conditions for a $10 \mathrm{MW}_{\mathrm{e}}$ waste heat recovery $\mathrm{S}_{-} \mathrm{CO}_{2}$ Brayton Power Cycle. These conditions correspond to Design Point \#1 ................................ 48

Table 4-2: Initial sizing dimensions. These parameters were used to generate the $1^{\text {st }} 3 \mathrm{D}$ geometry. As the 3D analysis was completed the geometry was modified several

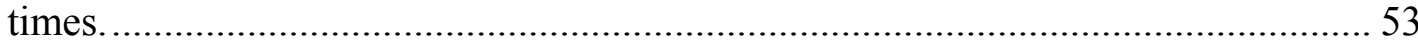

Table 4-3: Summary of Design Point \#2 ................................................................... 56

Table 4-4: Summary of Reynolds Number between Design Point \#1 and Design Point \#2.

Table 5-1: Summary of the information required by Turbo Setup................................... 61

Table 5-2: Summary of the initial geometry sizing parameters, the values presented below were used as inputs in BladeGen to generate the $1^{\text {st }}$ geometry........................62 62

Table 7-1: Non-dimensional dependent parameters Enthalpy Rise, Pressure Rise, Power coefficients and expressed for ideal gas behavior and real gas behavior. .............. 110

Table 7-2: Summary of the three different Projection Points used to create Figure 7-7.125

Table A-0-1: List of Recoverable Waste Heat Sources of High Temperature (Dincer and Zamfirescu, 2014). 


\title{
Nomenclature
}

\author{
$\underline{\text { Symbol }}$ \\ a \\ $\mathrm{C}$ \\ $C_{p}$ \\ $C_{p}$ \\ $C_{r}$ \\ $C_{v}$ \\ $C_{w}$ \\ $\mathrm{D}$ \\ $h$ \\ K \\ $\mathrm{L}$ \\ $\mathrm{M}$ \\ $\mathrm{Ma}$ \\ $\dot{m}$ \\ $\mathrm{N}$ \\ $n_{S}$ \\ $\mathrm{P}$ \\ $\mathrm{P}$ \\ $\Pi_{h}$ \\ $\Pi_{m}$ \\ $\Pi_{N}$ \\ $r$ \\ Re \\ $\mathrm{R}$ \\ $\mathrm{T}$ \\ TE \\ $\mathrm{U}$ \\ $v$ \\ $\mathrm{V}$ \\ $\dot{V}$ \\ W \\ W \\ W \\ $\mathrm{x}$ \\ $\mathrm{y}$ \\ $y^{+}$ \\ $\mathrm{z}$ \\ $Z$

\section{Definition} \\ Speed of sound $[\mathrm{m} / \mathrm{s}]$ \\ Absolute velocity $[\mathrm{m} / \mathrm{s}]$ \\ Specific heat capacity constant pressure $[\mathrm{kJ} / \mathrm{kg} \mathrm{K}]$ \\ Recovery pressure coefficient \\ Component of radial velocity $[\mathrm{m} / \mathrm{s}$ ] \\ Specific heat capacity constant volume $[\mathrm{kJ} / \mathrm{kg} \mathrm{K}]$ \\ Component of tangential velocity $[\mathrm{m} / \mathrm{s}]$ \\ Diameter [m] \\ Enthalpy $[\mathrm{J} / \mathrm{kg}]$ \\ Pressure loss coefficient \\ Blade chord [m] \\ Model \\ Mach number \\ Mass flow rate $[\mathrm{kg} / \mathrm{s}]$ \\ Rotational speed (RPM) \\ Isentropic exponent \\ Pressure [MPa] \\ Prototype \\ Enthalpy Rise Coefficient $\left(\Pi_{h}=\frac{\Delta h_{0}}{a_{o}^{2}}\right)$ \\ Flow Coefficient $\left(\Pi_{\mathrm{m}}=\frac{\dot{m} \sqrt{T_{o 1}}}{P_{o 1}} \sqrt{\frac{Z R}{n_{s}}} \frac{1}{D^{2}}\right)$ \\ Speed Coefficient $\left(\Pi_{\mathrm{N}}=\frac{N D}{a_{o}}\right)$ \\ Radius [m] \\ Reynolds Number \\ Gas constant [ $\mathrm{J} / \mathrm{K}$ kg] \\ Temperature $\left[\mathrm{K}\right.$ or $\left.{ }^{\circ} \mathrm{C}\right]$ \\ Trailing Edge \\ Blade speed $[\mathrm{m} / \mathrm{s}]$ \\ Specific volume $\left[\mathrm{m}^{3} / \mathrm{kg}\right]$ \\ Dynamic Viscosity $\left[\mathrm{cm}^{2} / \mathrm{s}\right]$ \\ Volume flow rate $\left[\mathrm{m}^{3} / \mathrm{s}\right]$ \\ Power [W] \\ Work [J] \\ Component of relative velocity $[\mathrm{m} / \mathrm{s}]$ \\ $\mathrm{X}$ coordinate $[\mathrm{m}]$ \\ Y Coordinate $[\mathrm{m}]$ \\ Non-dimensional distance from the wall \\ $\mathrm{Z}$ coordinate $[\mathrm{m}]$ \\ Compressibility factor $\left(z=\frac{P}{\rho R T}\right)$
}




\begin{tabular}{|c|c|}
\hline & Greek Symbols \\
\hline$\alpha$ & Flow angle $\left[{ }^{\circ}\right]$ \\
\hline$\beta$ & Blade metal angle $\left[{ }^{\circ}\right]$ \\
\hline$\gamma$ & Ratio of specific heat $\left(\frac{C p}{C_{v}}\right)$ \\
\hline$\Delta$ & Difference \\
\hline$\varepsilon$ & Percent error $(\%)$ \\
\hline$\eta$ & Efficiency $(\%)$ \\
\hline$\phi, \varnothing$ & Flow Coefficient $\left(\emptyset_{1}=\frac{\dot{V}_{0}}{\pi r_{2}^{2} U_{2}}\right)$ \\
\hline$\varphi_{2}$ & Flow Coefficient $\left(\varphi_{2}=C_{r_{2}} / U_{2}\right)$ \\
\hline$\mu$ & Dynamic viscosity $\left[\mathrm{Pa}^{*} \mathrm{~s}\right]$ \\
\hline$\pi$ & $\mathrm{Pi}$ \\
\hline$\rho$ & Density $\left[\mathrm{kg} / \mathrm{m}^{3}\right]$ \\
\hline$\psi$ & Work coefficient \\
\hline$\omega$ & Rotational speed $[\mathrm{Rad} / \mathrm{s}]$ \\
\hline & Subscripts \\
\hline 0 & Inlet to Inlet Guide Vanes \\
\hline 1 & Impeller inlet \\
\hline 2 & Impeller outlet \\
\hline 3 & Diffuser outlet \\
\hline o & Stagnation conditions \\
\hline actual & Actual compression work \\
\hline $\mathrm{c}$ & Critical point conditions \\
\hline Comp & Compressor \\
\hline ideal & Isentropic compression \\
\hline DP & Design point \\
\hline$m f r$ & CFD output variable - mass flow $[\mathrm{kg} / \mathrm{s}]$ \\
\hline reference & Reference state point \\
\hline SP & Surge point \\
\hline & Superscript \\
\hline ' & Ideal process \\
\hline & Abbreviations \\
\hline $\mathrm{CP}$ & Critical Point \\
\hline DP & Design Point \\
\hline EOS & Equation of State \\
\hline LE & Leading Edge \\
\hline LHS & Left Hand Side \\
\hline NIST & National Institute of Standards and Technology \\
\hline RANS & Reynolds-Averaged Navier-Stokes \\
\hline RGP & Real Gas Property File \\
\hline $\mathrm{S}-\mathrm{CO}_{2}$ & Supercritical Carbon Dioxide \\
\hline $\mathrm{TE}$ & Trailing Edge \\
\hline
\end{tabular}




\section{Chapter 1}

\section{Introduction}

\subsection{Background and Motivation}

The concept of power cycles using carbon dioxide as a working fluid dates to 1948 when Sulzer Bros filled a patent for a partial condensation $\mathrm{CO}_{2}$ Brayton cycle (Dostal, 2004). However, recent interest was sparked again by a study highlighting the benefits of a supercritical carbon dioxide $\left(\mathrm{S}_{-} \mathrm{CO}_{2}\right)$ Brayton cycle in nuclear reactors (Dostal, 2004). Figure 1-1 illustrates a simple regenerative Brayton cycle. Brayton cycles are a type of power system in which, in the simplest form, fluid is compressed to a certain pressure, heat is added at a constant pressure and the fluid is then expanded in the turbine.

In the regenerative cycle, heat is recuperated prior to entering the main heat exchanger.

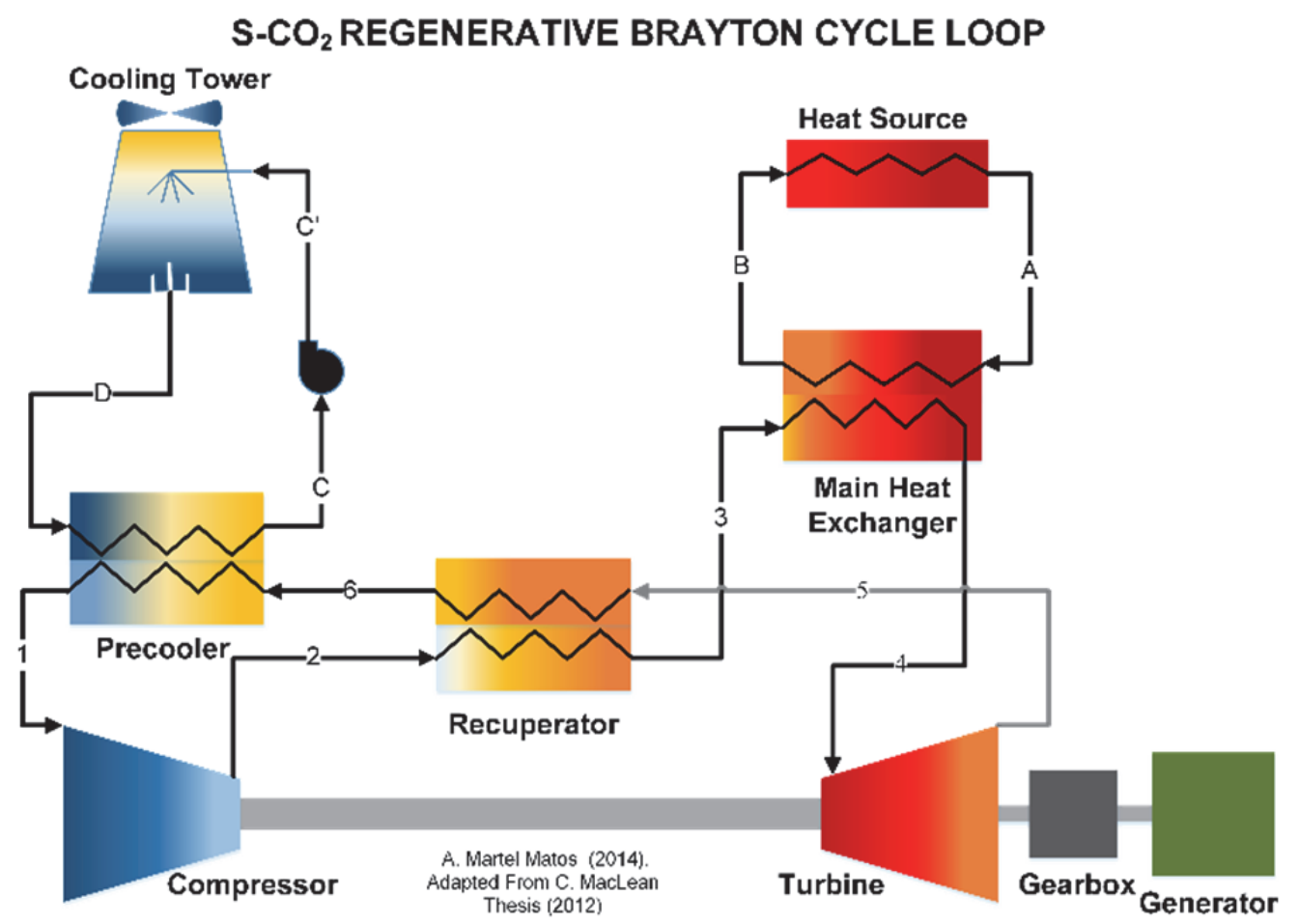

Figure 1-1:Diagram of a Simple Regenerative Brayton Cycle. 
One of the main benefits of using $\mathrm{S}-\mathrm{CO}_{2}$ in the Brayton cycle is the increase in cycle efficiency. Studies estimate that cycle efficiency can be as high as 50\% (SANDIA, 2013), making them highly competitive in comparison to cycles which use conventional steam or Helium. A secondary benefit of $\mathrm{S}-\mathrm{CO}_{2}$ is associated with its low critical temperature $(304.13 \mathrm{~K})$. This temperature is well within reach of ambient temperature and is low compared to other working fluids. By operating near the critical point (304.13 $\mathrm{K}, 7.37 \mathrm{MPa}$ ), and at a supercritical level, the cycle benefits from the fluid properties. The high density of the fluid at supercritical state allows the design of smaller turbomachinery compared to other cycles which use air or steam. The high density allows the compressor to behave more as a pump and therefore less work is required to achieve a desired pressure rise. Similarly, SANDIA (2013) have shown that these systems have a smaller footprint, which is directly related to a reduction in costs (Wright, 2016).

Brayton cycle operating with $\mathrm{S}-\mathrm{CO}_{2}$ provide the flexibility to be paired with different heat sources such as fossil, nuclear, renewables and waste heat. The vast range of heat sources allow this power cycle to be designed to meet different power ratings ( 1 $\mathrm{MW}_{\mathrm{e}}, 10 \mathrm{MW}_{\mathrm{e}},>100 \mathrm{MW}_{\mathrm{e}}$ ). Waste heat recovery is a vast and relatively untapped energy source. A market report from Market and Markets (2016) forecasts that the waste heat recovery market in the US will reach a market value of $\$ 53.1$ Billion for 2018 and is expected to reach $\$ 65.87$ Billion by 2021 .

\subsection{Challenges}

Designing the components for these cycles is complicated due to the fluid properties of $\mathrm{S}-\mathrm{CO}_{2}$. Conventional design methods may not necessarily provide efficient 
designs. Currently, little is known regarding supercritical fluids and their behavior in the internal flow of the turbomachinery (Baltadjiev, 2012), specifically in the compressor. The compressor design point tends to be located near the critical point. Within this region and around the pseudocritical region, fluid properties experience sharp changes with very small changes in pressure and temperature. In this region, the fluid behaves as a non-ideal fluid, and hence the ideal gas assumptions are not valid. This results in the having to employ an Equation of State that can accurately predict the thermodynamic properties. So far, very few investigations have focused on validating the accuracy of the existing design methods and numerical values for the design of air compressors for use as $\mathrm{S}-\mathrm{CO}_{2}$ compressors. The designer not only faces challenges during the design phase and application of the design methods, but also faces challenges during the computational modelling and in the use of computational fluid dynamics (CFD). Several researchers have highlighted the difficulties in achieving proper convergence (Baltadjiev 2014, Pham et al 2016). Another issue influencing the computation and convergence is the appearance of condensation and the capabilities of CFD to properly model such phenomena. These topics are further discussed in the following chapters.

\subsection{Scope and Objectives}

The goal of this project was to develop the preliminary aerodynamic design of a centrifugal compressor for a $10 \mathrm{MW}_{\mathrm{e}}$ waste heat recovery supercritical carbon dioxide $\left(\mathrm{S}-\mathrm{CO}_{2}\right)$ Brayton power plant. The project completed an aerodynamic study via computational fluid dynamics using ANSYS CFX V17.2. The results summarize the final preliminary geometry of the compressor and diffuser, the 3D aerodynamic results and the 
compressor performance maps. This research project was motivated and funded by Doosan Heavy Industries \& Construction Co. Ltd. (the industrial partner). Due to the needs of the industrial partner, the study was subjected to several deadlines and challenges that had to be solved within a time constraint.

\subsection{Key Contributions}

The performance of compressors is expressed through performance maps. For ideal gas flows, the performance of the machine is expressed by one map in terms of corrected mass flow versus pressure ratios for given corrected speed lines. However, when using supercritical non-ideal fluids, several maps are required to fully expressed the performance of the machine. The thermodynamic properties of supercritical real gas flows are a function of temperature and pressure. Near the critical point, the properties experience sharp changes with small changes in pressure and temperature. The sharp changes in this region suggest that the performance of the machine is highly dependent on these changes which underlies the need for multiple performance map.

The thesis's contribution is to provide a tentative solution to the necessity of multiple compressor maps for non-ideal gas flows. By expressing the performance map in terms of the Flow Coefficient versus Enthalpy Rise Coefficient for different corrected speed lines, one can express the performance of the machine within a single map, referred to as the Generalized Compressor Map. The investigation validated the Generalized Compressor Map for two different fluids down to a compressibility factor of $Z=0.65$. Further investigation is required near the critical point to validate the Generalize Compressor Map for low values of $\mathrm{Z}$. 
This investigation also presents the Projection Methodology. This methodology uses similarity and scaling to create the corresponding compressor map near the critical point. However, the accuracy to which the maps are created near the critical point still needs further investigation. Just like the Generalize Compressor Map, this methodology has been validated down to a compressibility factor of $Z=0.65$.

\subsection{Roadmap}

- Chapter 2 focuses on the literature review. It presents a summary of common guidelines for the design of centrifugal compressors. It introduces the properties of $\mathrm{CO}_{2}$ near the critical point, highlights the sharp changes they undergo and the need to use a proper equation of state. This chapter also introduces dimensional analysis for compressible non-ideal flows.

- Chapter 3 describes the design process, clarifying the difference between the original design point and the partially dynamically similar design point.

- Chapter 4 presents the Design Point \#1, the initial sizing, and introduces the Scaling Methodology.

- Chapter 5 presents the different software packages from ANSYS Workbench that were used in this project.

- Chapter 6 present the results of the 3D aerodynamic analysis, and compare the initial geometry to the final preliminary geometry.

- Chapter 7 introduces the Enthalpy Rise Coefficient $\left(\Pi_{\mathrm{h}}\right)$, the Projection Methodology as well as the final compressor performance maps corresponding to Design Point \#1.

- Chapter 8 presents the conclusions and future work. 


\section{Chapter 2}

\section{Literature Review}

\subsection{Introduction}

Chapter 2 covers several fundamentals required for proper understanding of this thesis. Section 2.2 to Section 2.6 provides background information to $\mathrm{S}-\mathrm{CO}_{2}$ Brayton cycles and waste heat recovery systems. Section 2.7 to Section 2.10 describes the centrifugal compressor and reviews the methodologies and design guidelines used in the preliminary design. Section 2.11 discusses the need to treat $\mathrm{S}-\mathrm{CO}_{2}$ as a real gas and the influence this has on its fluids properties. Section 2.12 presents a set of non-dimensional parameters that describe compressible flow of non-ideal gases.

\subsection{Brayton Cycle}

As mentioned in Chapter 1 Brayton cycles are a type of thermodynamic power cycle. They usually operate at higher temperatures than Rankine cycles and therefore achieve higher efficiencies. Using supercritical carbon dioxide as the working fluid highly enhances the performance of the Brayton cycle. A simple regenerative Brayton Cycle was illustrated in Figure 1-1. The cycle configuration can be modified to incorporate reheating, intercooling or recompression among others. These modifications further improve the performance of the cycle. Several proposed Brayton cycles were investigated by Dostal (2004). A more recent study, presented in two parts by Crespi et. al (2017a) and (2017b), has catalogued many of the proposed cycles for $\mathrm{S}^{-\mathrm{CO}_{2}}$ into 
groups. The study further presented the optimal operating conditions of each proposed cycle.

\subsection{Benefits of Supercritical Carbon Dioxide Brayton Power Cycles}

As previously mentioned, the attractiveness of $\mathrm{S}-\mathrm{CO}_{2}$ cycles can be attributed to three main reasons; higher cycle efficiencies, a low critical temperature, and because of the supercritical state, components can be smaller than competing cycles.

\subsubsection{Expected Efficiency and Temperature Operating Range}

The expected high efficiencies in comparison to the competing Rankine and Helium cycles are illustrated in Figure 2-1. Current research indicates that $\mathrm{S}-\mathrm{CO}_{2}$ Brayton cycles have the capability to be designed for different source temperatures, ranging between $250^{\circ} \mathrm{C}$ to $750{ }^{\circ} \mathrm{C}$.

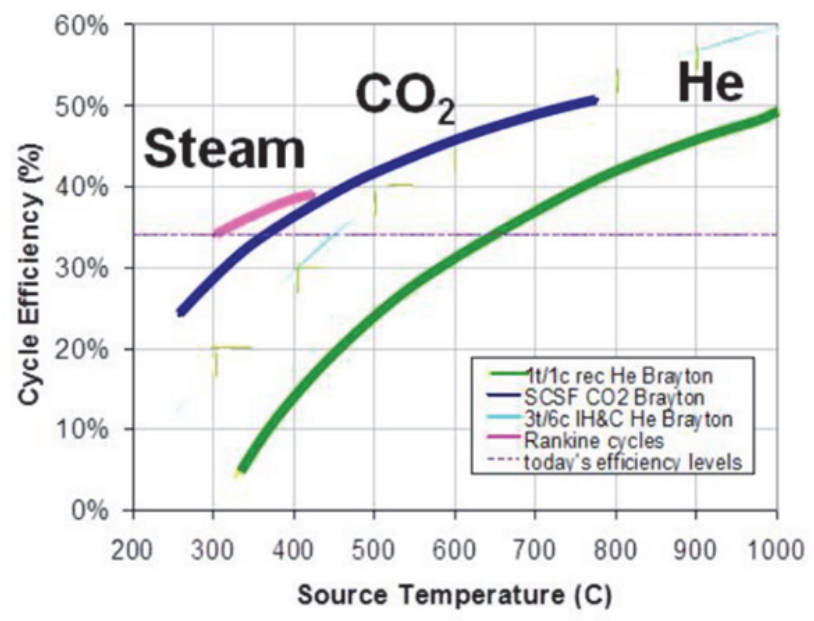

Figure 2-1: Cycle Efficiencies vs Source Temperature for fixed component efficiency (SANDIA, 2013).

This temperature range is beneficial because it allows $\mathrm{S}-\mathrm{CO}_{2}$ systems to be suitable for many applications such as nuclear, fossil fuel, and waste heat recovery, 
among other applications as shown in Table 2-1. This provides the flexibility of designing $\mathrm{S}-\mathrm{CO}_{2}$ Brayton cycles that act either as a primary power cycle or as a secondary, as is the case with waste heat recovery. Notice that waste heat recovery power plants can range between $1 \mathrm{MW}_{\mathrm{e}}, 10 \mathrm{MW}_{\mathrm{e}}$ and $100 \mathrm{MW}_{\mathrm{e}}$.

Table 2-1: Expected operating conditions for the $\mathrm{S}-\mathrm{CO}_{2}$ power cycles (Adapted from Musgrove, 2016).

\begin{tabular}{|l|c|c|c|}
\hline \multicolumn{1}{|c|}{ Application } & Size $\left[\mathrm{MW}_{\mathrm{e}}\right]$ & $\mathrm{Temperature}\left[{ }^{\circ} \mathbf{C}\right]$ & Pressure [Bar] \\
\hline Nuclear & $300-1000$ & $400-800$ & 350 \\
\hline Fossil Fuel & $500-1000$ & $550-1200$ & $150-350$ \\
\hline $\begin{array}{l}\text { Concentrated } \\
\text { Solar Power }\end{array}$ & $10-100$ & $500-800$ & 350 \\
\hline $\begin{array}{l}\text { Waste Heat } \\
\text { Recovery }\end{array}$ & $1,10,100$ & $230-650$ & $15-350$ \\
\hline Geothermal & $1,10,50$ & $100-300$ & 150 \\
\hline
\end{tabular}

It is important to highlight some of the current limitations. Table 2-1 presents temperature reaching as high as $1200{ }^{\circ} \mathrm{C}$ and $>30 \mathrm{MPa}$. Currently, the combination of high temperature and pressure imposes a current constraint as there are no commercially available materials that can withstand these conditions. However, it is expected that newer materials will allow for this combination of temperature and pressure to be reached.

\subsubsection{Size of Components}

Research has shown that turbomachinery can be $1 / 100^{\text {th }}$ the size of a steam turbines, while maintaining the same rated power (Drew, 2012). Figure 2-2 illustrates the different sizes of turbines corresponding to steam, helium and $\mathrm{CO}_{2}$ power cycles. A similar trend is observed in compressor sizing. The fluid properties at supercritical state provide a high- 
energy density allowing the creation of more compact heat exchangers (Musgrove, 2016).

Figure 2-3 illustrates the size difference between a steam condenser and a cooler using supercritical carbon dioxide.

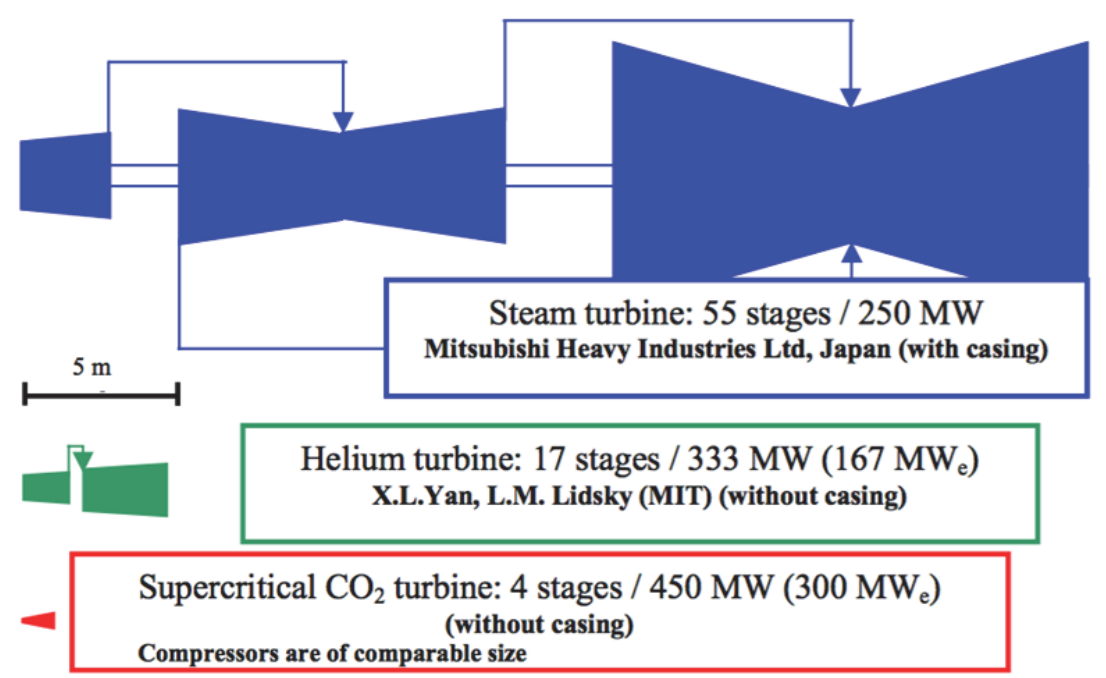

Figure 2-2: Comparison of turbine sizes for power cycles using different fluids (Dostal, 2004).

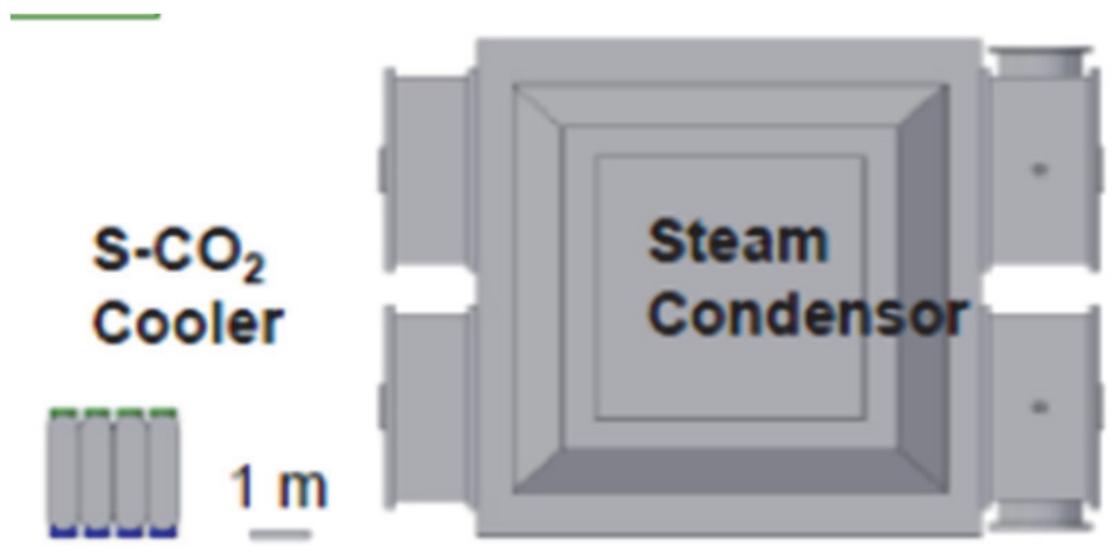

Figure 2-3: Comparison of the size of heat exchangers between $\mathrm{S}-\mathrm{CO}_{2}$ and steam (Musgrove, 2016). 


\subsection{Focus of Current Research}

Currently the applications that are receiving the most attention and funding are concentrated solar power (CSP), nuclear power, fossil fuel and waste heat recovery systems. S- $\mathrm{CO}_{2}$ Brayton cycles are attractive for $\mathrm{CSP}$ applications because of their compact size which allows engineers to optimize space and build the system by modules (Bauer, 2016). The $\mathrm{S}-\mathrm{CO}_{2}$ Brayton cycles are considered a strong candidate for the next generation of nuclear reactors because of their overall smaller size, as well as their higher expected efficiencies (Dostal, 2004). As mentioned before, and as a focus of this project, waste heat recovery is a big area of research. The possibility of having different power output makes waste heat recovery units attractive, both as a demonstration of the technology as well as a suitable size to enter the market (Rochau, 2016).

\subsection{Motivation for the Design of a $10 \mathrm{MW}_{\mathrm{e}}$ System}

Sandia National Laboratories (SNL) has identified intermediate size power systems as the most suitable for demonstration facilities. Intermediate size power facilities provide a maximum benefit when the component technologies used matched those that will be used in the full scale, commercial system (SANDIA, 2013). SNL has suggested that a $10 \mathrm{MWe}$ system would be an attractive intermediate scale demonstrator (SANDIA, 2013). Figure 2-4 illustrates the different options for components available to be used by $\mathrm{S}-\mathrm{CO}_{2}$ Brayton Cycles. From the figure, it is possible to see how a $10 \mathrm{MWe}$ system can use components which can be scaled to larger-scale facilities such as $100 \mathrm{MWe}$ and $300 \mathrm{MWe}$ power plants. Constructing a 10 MWe demonstration facility can showcase several features of the technology, such as to proving that the current theoretical efficiencies are achievable. 
It can also demonstrate the performance and durability of components and materials. Similarly, it can provide great value in developing the control systems capabilities. Therefore, designing a $10 \mathrm{MW}_{\mathrm{e}}$ system provides great value towards the development of the technology.

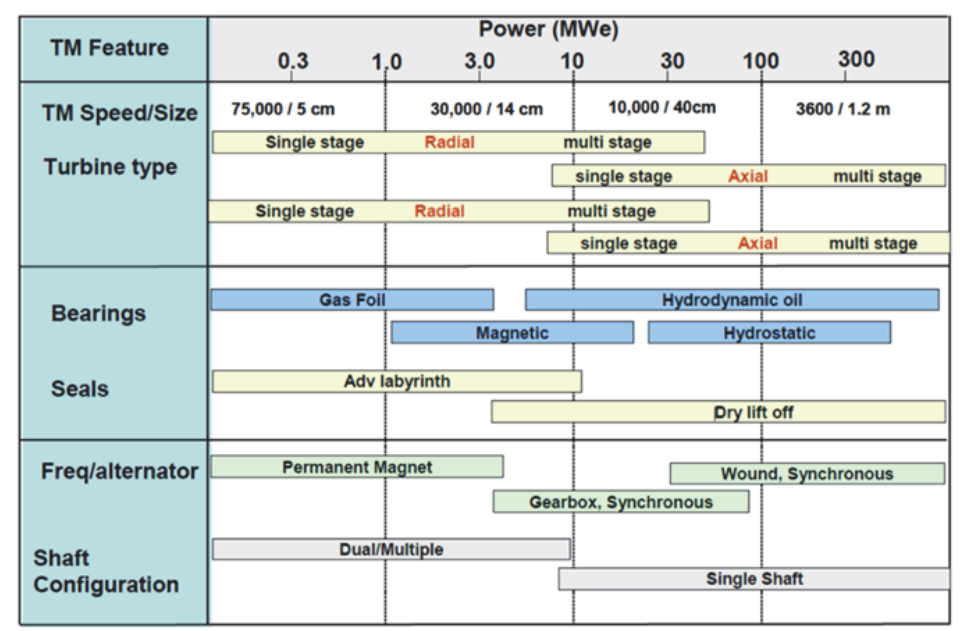

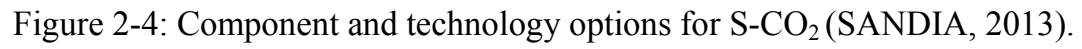

\subsection{Waste Heat Recovery (WHR)}

Waste heat recovery systems are important to $\mathrm{S}-\mathrm{CO}_{2}$ Brayton cycles for two reasons. First, is that they have been identified by studies as having an attractive size (SANDIA, 2013). Second, waste heat has been identified as one of the first markets that $\mathrm{S}-\mathrm{CO}_{2}$ Brayton cycles technologies should penetrate (Rochau, 2016).

Waste heat is referred to as the un-used energy that is rejected from a thermodynamic system. The exhausted energy can be released from industrial processes or from power systems such as fossil fuel and nuclear. The wasted energy can amount to $20 \%$ to $50 \%$ of the energy consumed by the system (U.S Department of Energy, 2008). Table 2-2 tabulates various power generation systems and the corresponding efficiencies. It is 
estimated that between $167 \mathrm{GW}$ to $436 \mathrm{GW}$ remains unrecovered from systems in the US (U.S Department of Energy, 2008).

Table 2-2: Typical thermal efficiencies for power generation options, adapted from (Suppes and Storvick, 2015).

\begin{tabular}{|l|c|}
\hline \multicolumn{1}{c}{ Application } & Efficiency \\
\hline Natural Gas - Peak Power Turbine & $25 \%$ \\
\hline Nuclear Power & $30 \%-33 \%$ \\
\hline Coal - Steam Cycle & $38 \%-45 \%$ \\
\hline Nuclear - Pebble Bed Modular Reactor & $45 \%$ \\
\hline Coal - Gasification Combined Cycle & $48 \%$ \\
\hline Natural Gas - Combined Cycle & $50 \%-60 \%$ \\
\hline Future (2020) - Fuel Cell Combined Cycle & $70 \%$ \\
\hline
\end{tabular}

For further information see Appendix A: List of Identified Waste Heat Sources presents a list of some of the identified waste heat sources and indicates the operating temperatures.

\subsubsection{Market Entry}

Sandia National Laboratories have identified waste heat as one of the suitable markets for which S-CO $\mathrm{CO}_{2}$ Brayton cycles can first be commercialized in (Rochau, 2016). A market report from Market and Markets (2016) forecasts that the waste heat recovery market in the US will reach a market value of \$53.1 Billion for 2018 and is expected to reach $\$ 65.87$ Billion by 2021 . Thermo-economic analysis of waste heat recovery power cycles using $\mathrm{S}-\mathrm{CO}_{2}$ Brayton cycles have been completed by SuperCritical Technologies Inc. (Wright, 2016). 


\subsection{Gas Turbine Design Process -Preliminary Design}

Saravanamuttoo (2009) presents a road map for the gas turbine design process, beginning with market research continuing until after sales services. The focus of this thesis is explicitly on the preliminary design of the centrifugal compressor. It is important to understand what exactly a preliminary aerodynamic design involves. Figure 2-5 illustrates the gas turbine design process, and the red rectangle highlights the scope under which this project falls.

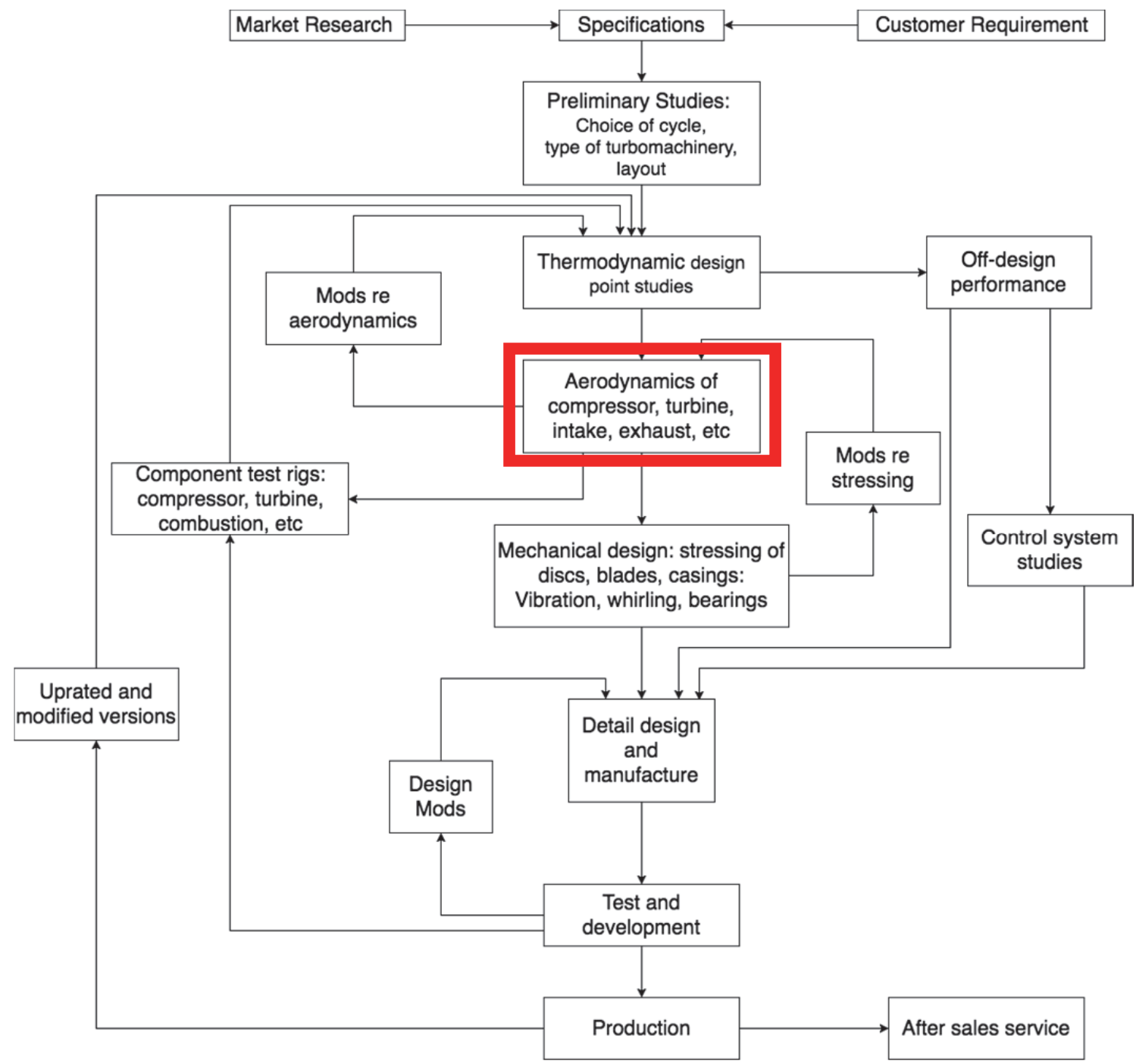

Figure 2-5: Gas Turbine Design Process (Saravanamuttoo, 2009). 


\subsection{Centrifugal Compressors}

Centrifugal compressors, commonly referred to as radial compressors, are turbomachines responsible for transferring mechanical work into the fluid. The transfer of mechanical work to the system is expressed as a pressure rise. Figure 2-6 illustrates the four different components comprising a centrifugal compressor; inlet guide vanes (IGV) which are optional, the impeller, the diffuser and the volute. Note that this investigation will focus solely on the design of the impeller and diffuser.

The IGV are responsible for smoothly guiding the flow into the impeller, although they are not commonly used (Sjolander, 2017). The impeller is the only rotating component, and it provides the total pressure rise to the fluid. The diffuser contributes to a reduction in kinetic energy and therefore an increase in the static pressure. The volute collects the fluid coming out of the diffuser, further reduces the kinetic energy of the flow and guides the flow to the outlet pipe.

Notice the numbering in Figure 2-6, the inlet to the IGV is numbered as 0 , the inlet to the impeller as 1 , the outlet of the impeller and consequently the inlet to the diffuser as 2 . The outlet of the diffuser is numbered as 3 and consequently the inlet to the volute is also 3. This numbering system will be used throughout the thesis. 


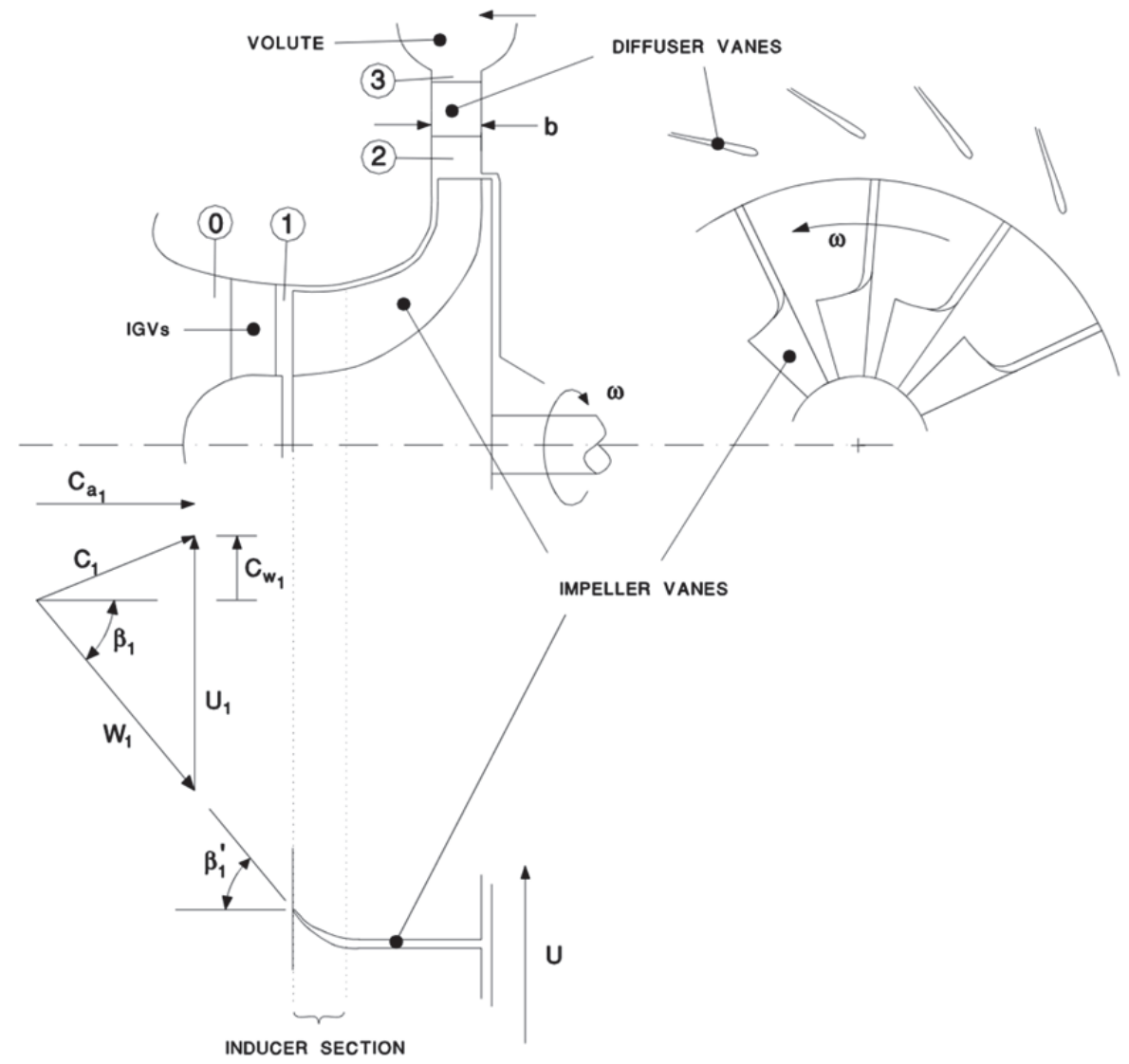

Figure 2-6: Centrifugal compressor (Sjolander, 2015).

It was previously mentioned that the transfer of mechanical work to the system is expressed as a pressure rise. Therefore, it is possible to say that that $\Delta \mathrm{P}_{0}$ or $\mathrm{P}_{0, \text { In }} / \mathrm{P}_{0, \text { Out }}$ is a function of $\Delta \mathrm{h}_{0}$ and efficiency $(\eta)$. The actual total enthalpy rise $\left(\Delta \mathrm{h}_{0}\right)$ of the compressor is defined by the Euler equation, as shown by Equation 2.1 .

$$
\frac{\dot{W}_{\text {Shaft }}}{\dot{m}}=\Delta h_{o}=U_{2} C_{w_{2}}-U_{1} C_{w_{1}}
$$

The efficiency $(\eta)$ of compressors, either centrifugal or radial, is defined as the ratio of the ideal work to the actual work required by the machine to achieve the same pressure rise. Therefore, the efficiency is defined as shown in Equation 2.2.

$$
\eta_{\text {comp }}=\frac{\dot{W}_{\text {ideal }}}{\dot{W}_{\text {actual }}}=\frac{\Delta h_{0}^{\prime}}{\Delta \mathrm{h}_{0}}=\frac{h_{2}^{\prime}-h_{1}}{h_{2}-h_{1}}
$$


From the pressure ratio one can calculate the ideal total enthalpy rise $\left(\Delta h_{0}^{\prime}\right)$. Figure 2-7 illustrates the ideal $\left(\Delta h_{0}^{\prime}\right)$ and actual $\left(\Delta \mathrm{h}_{0}\right)$ compression processes that provide the same pressure ratio $\left(\mathrm{P}_{\mathrm{o} 2} / \mathrm{P}_{01}\right)$.

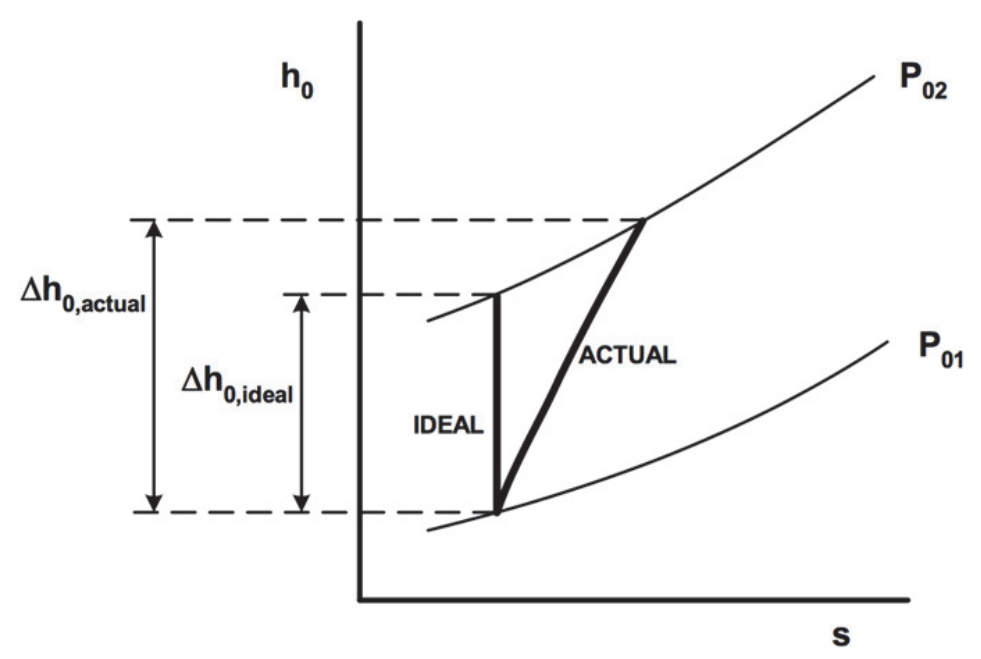

Figure 2-7:Total Enthalpy vs Entropy diagram $\left(\mathrm{h}_{0}-\mathrm{s}\right)$. Illustrates the ideal and actual compression processes that provide the same pressure ratio $\left(\mathrm{P}_{\mathrm{o} 2} / \mathrm{P}_{01}\right)$ (Sjolander, 2015).

\subsection{Initial Sizing of Centrifugal Compressors}

The initial sizing of impellers is commonly done using empirical correlations that have been derived from well-designed machines. In the $\mathrm{S}^{-\mathrm{CO}_{2}}$ community, one of the most popular initial sizing methodologies is the use of the specific speed $(\Omega)$ - specific diameter $\left(D_{s}\right)$. This method has been employed by (Fuller, 2012) to size and design the SANDIA compressor (Wright 2010 \& SANDIA, 2013). Similarly, this method has also been used by (Monje, 2014). The initial sizing of this machine was based on specific speed and the initial efficiency selection (Shepherd, 1956 \& Sjolander, 2015). Similarly, the use of the Flow $(\phi)$ and Work $(\psi)$ coefficient (Aungier, 2000) was used to double check the initial estimate for more detail see Appendix B: Initial Sizing Using the Flow $(\phi)$ and Work $(\psi)$ Coefficient. A parametric analysis was performed using a code 
provided by the client. It is important to understand that this initial sizing and parametric study was not done by the author of this thesis. This investigation solely used the parameters derived from the initial sizing (Kibsey, 2016).

\subsubsection{De Haller Number}

In 1953, De Haller introduced a simple measure to quantify the overall diffusion inside the impeller (Dixon, 2014). The amount of diffusion has wide impact on the quality of the flow inside the passage. Large amount of diffusion can cause a large increase in static pressure, which can affect the boundary layer. If the boundary layer separates, then stalling of the passage or entire compressor may occur. Therefore, it is necessary to limit the overall diffusion inside the impeller. The De Haller Number is defined as the ratio between the impeller outlet relative velocity $\left(\mathrm{W}_{2}\right)$ and the impeller inlet relative velocity $\left(\mathrm{W}_{1}\right)$ as shown in Equation 2.3. The De Haller Number is not a specific value and several authors recommend different values as shown in Table 2-3.

$$
\text { De Haller }=\frac{W_{2}}{W_{1}}
$$

Table 2-3: Literature recommended De Haller values

\begin{tabular}{|c|c|c|}
\hline Author & \multicolumn{2}{|c|}{ Recommended De Haller Value } \\
\hline \multirow{2}{*}{ Aungier (2000) * } & Recommended & $W_{2} / W_{1}>0.75$ \\
\hline & Never Exceed & $W_{2} / W_{1}<0.65$ \\
\hline Wilson \& Korakianitis (1978) * & Recommended & $W_{2} / W_{1}>0.8$ \\
\hline Rodgers (1978) * & Recommended & $W_{2} / W_{1}>0.71$ \\
\hline \multirow{2}{*}{ Yoshinga (1982) * } & Low PR - Compressors \& Fans & $W_{2} / W_{1}>0.8$ \\
\hline & High PR - Compressors $(\mathrm{PR}=8)$ & $W_{2} / W_{1}>0.6$ \\
\hline De Haller (1953) ** & Recommended & $W_{2} / W_{1}>0.72$ \\
\hline
\end{tabular}




\subsubsection{Flow Coefficient $\left(\boldsymbol{\varphi}_{2}\right)$ - Limits of the Surge and Choke Region}

In 1980, Eckardt presented a study on the flow measurements of a centrifugal compressor. The study investigated two types of compressors, one with radial vanes $\left(90^{\circ}\right)$ and another with a backsweep of $60^{\circ}$. The results of the operating region were expressed in terms of the Slip Factor $(\mu)$ versus Flow Coefficient $(\varphi)$ as shown in Figure 2-8. Slip factor is commonly defined as the ratio of the actual tangential component of absolute velocity $\left(\mathrm{C}_{\mathrm{w}}\right)$ to the ideal tangential component of absolute velocity $\left(\mathrm{C}_{\mathrm{w}}^{\prime}\right)$. In essence, it is a ratio measuring the angle deviation between the flow and the blade.

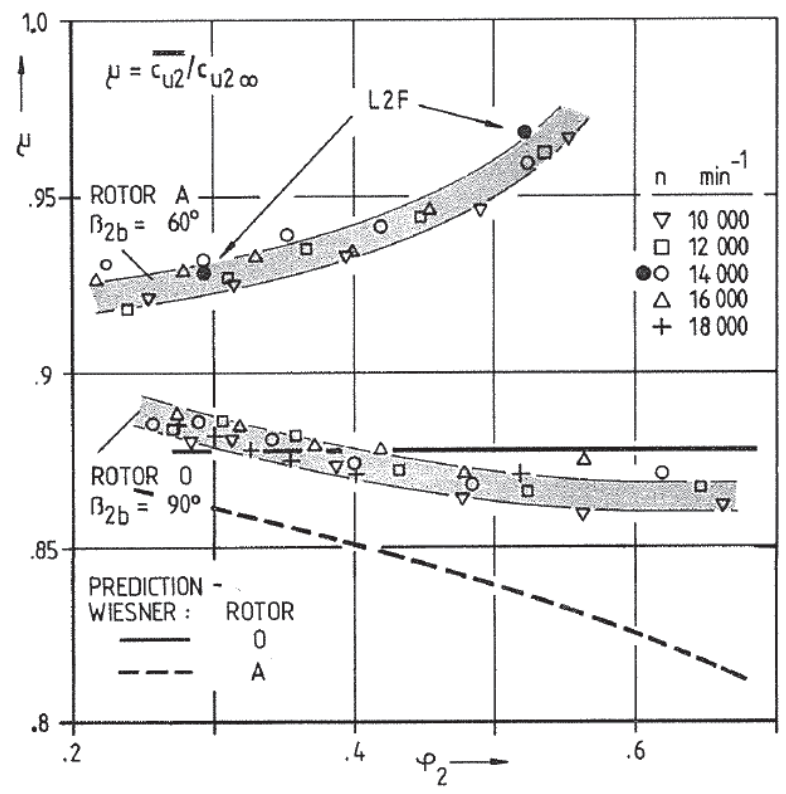

Figure 2-8: Variation of Slip Factor $(\mu)$ vs Flow Coefficient ( $\varphi)$ (Eckardt 1980).

Equation 2.4 defines the Flow Coefficient $\left(\varphi_{2}\right), U_{2}$ is the tip blade speed. $C_{r_{2}}$ is the radial velocity at the impeller outlet and is controlled by continuity as expressed by Equation 2.5.

$$
\varphi_{2}=C_{r_{2}} / U_{2}
$$

where: 


$$
C_{r_{2}}=\frac{\dot{m}}{\rho_{02} 2 \pi b_{2}}
$$

From Figure 2-8 one can observe a trend regarding the Flow Coefficient $\left(\varphi_{2}\right)$. It is possible to observe that both impellers $\left(90^{\circ}\right.$ and $\left.60^{\circ}\right)$ approach surge near a Flow Coefficient value of $\sim 0.20$ to $\sim 0.25$. A similar trend is observed as the impellers approach choking. The $90^{\circ}$ impeller appears to have a wider range of operation, reaching the choke region near a value of $\sim 0.65$, while the $60^{\circ}$ reach the choke region at $\sim 0.55$. From this observation, a designer can guide the initial design of a compressor to achieve similar values of the outlet Flow Coefficient. These values are a good first estimate guideline when deriving the characteristic maps of the machine from CFD. From Figure 2-8, it can be said that a well-designed geometry will probably have a design operating point between of $\sim 0.35$ and $\sim 0.4$. A Flow Coefficient around this region should provide enough surge margin.

\subsubsection{Mass Flow Surge Margin}

The surge margin can be expressed in different forms. In this investigation, it will be described in terms of mass flow as shown by Equation 2.6. The surge margin represents the amount of mass flow that can be reduced before the compressor reaches the surge region. The subscripts DP correspond to Design Point and SP correspond to Surge Point.

$$
\text { Mass Flow Surge Margin }=\frac{\left(\dot{m}_{D P}-\dot{m}_{S P}\right)}{\dot{m}_{D P}} * 100 \%
$$




\subsubsection{Vaneless Diffuser: Sizing and Pressure losses}

Aungier (1995) presents an empirical relation to help designers size a vaneless diffuser as shown in Equation 2.7, where $\emptyset_{1}$ is the Flow Coefficient at the impeller inlet as shown by Equation 2.8, and $r_{2}$ is the impeller outlet radius.

$$
\begin{gathered}
r_{3}=\left(\left(1.55+\emptyset_{1}\right) * r_{2}\right) \\
\emptyset_{1}=\frac{\dot{V}_{0}}{\pi r_{2}^{2} U_{2}}
\end{gathered}
$$

Diffusers can be of several shapes, such as conical or constant width as shown in Figure 2-9 or a "pinched" diffuser as shown in Figure 2-10.

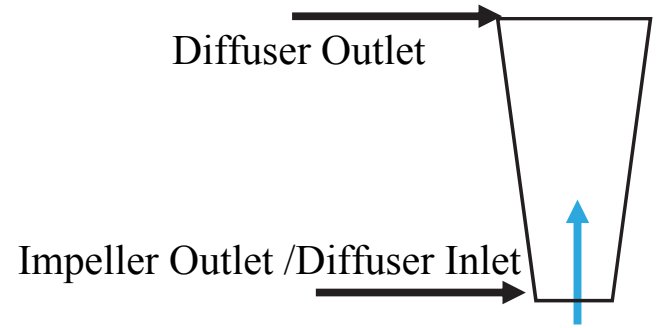

(a)

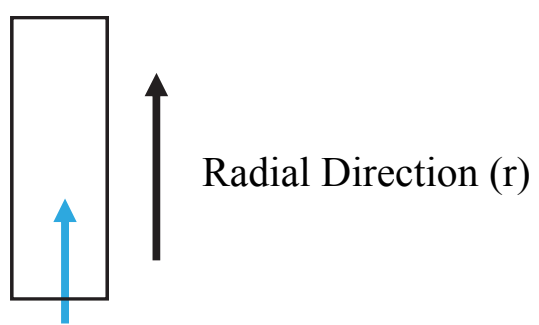

(b)

Figure 2-9: Types of vaneless diffusers (a) conical diffuser (expanding) diffuser, (b) constant width diffuser.

In the case where a vaneless diffuser is to be pinched, Aungier suggests that the width reduction (b) be made on the shroud side rather than the hub side. Figure 2-10 illustrates different degrees of pinched diffuser designs. 


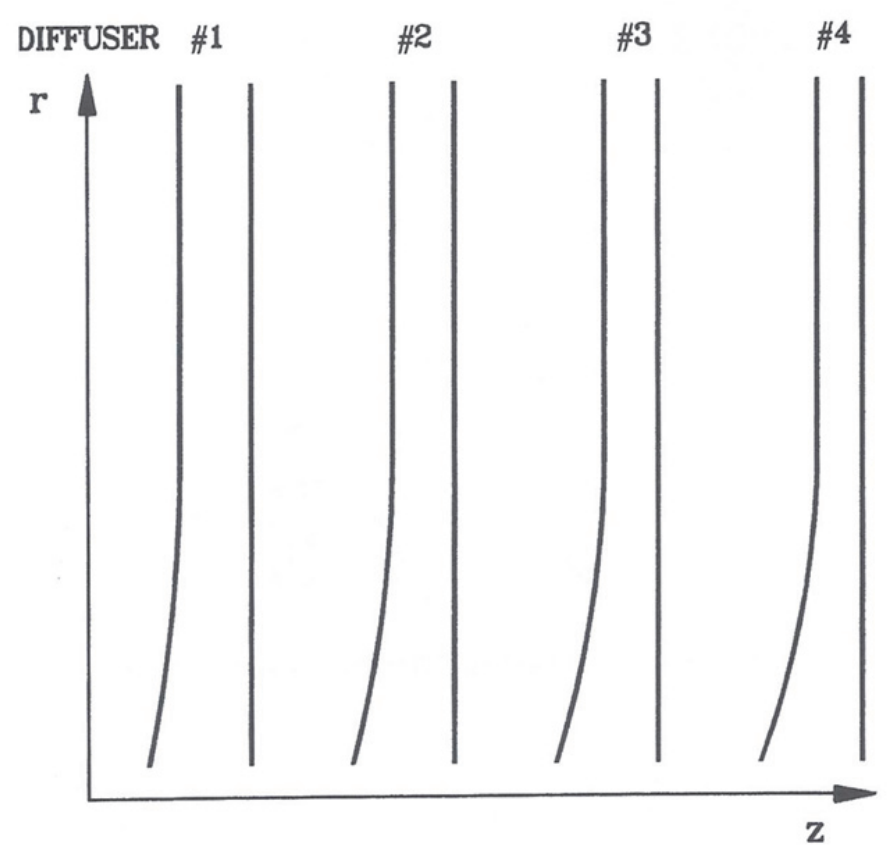

Figure 2-10: Different degrees of "pinched" vaneless diffusers (Aungier, 2000).

It is common practice to analyze diffusers in terms of the recovery pressure coefficient (Cp) and total pressure loss (k). Japikse and Baines (1997) present the definition of each parameter as shown in Equation 2.9 and Equation 2.10.

$$
\begin{gathered}
C_{p}=\frac{\left(p_{3}-p_{2}\right)}{\left(p_{0_{2}}-p_{2}\right)} \\
k=\frac{p_{o_{2}}-p_{o_{3}}}{p_{o_{2}}-p_{2}}
\end{gathered}
$$

where $p$ corresponds to the static pressure, $p_{0}$ corresponds to the total pressure.

\subsection{Properties of Carbon Dioxide $\left(\mathrm{CO}_{2}\right)$}

There are two important conditions to be understood about supercritical fluids. The first is that they experience sharp changes in properties near the critical point. This introduces a level of complexity when modelling or numerically analyzing systems. since properties vary sharply with very small changes in pressure and temperature, 
convergence tends to be challenging. Second, the flow behaves as a real gas. This implies that the ideal gas law is not valid and therefore a proper Equation of State is necessary to properly define the properties.

As previously mentioned, a benefit of using Carbon Dioxide as the working fluid is that it has a low critical temperature (near ambient) in comparison to other fluids as shown in Table 2-4.

Table 2-4: Summary of Critical temperature and pressure for different fluids (REFPROP, 2015).

\begin{tabular}{|l|c|c|}
\hline Fluid & Critical Temp [K] & Critical Pressure [MPa] \\
\hline $\mathbf{C O}_{2}$ & 304.13 & 73.773 \\
\hline Ammonia & 405.40 & 11.333 \\
\hline Water & 647.10 & 22.064 \\
\hline Air & 140.73 & 37.25 \\
\hline Helium & 5.19 & 0.22761 \\
\hline
\end{tabular}

The fluid properties at supercritical state are beneficial to the system due to the previously mentioned reasons (efficiency, and high-density). It is important to understand that the benefits of supercritical properties can be exploited for other fluids, however, the low critical temperature of $\mathrm{CO}_{2}$ is what makes it an attractive fluid for this purpose.

Substances are at a supercritical state when they have reached and/or surpass their corresponding critical point pressures and temperatures. The critical temperature of a pure substance is defined as the maximum temperature at which liquid and vapor phases can coexist in equilibrium (Moran \& Shapiro, 2011). Figure 2-11 illustrates the phase diagram of $\mathrm{CO}_{2}$ in which the supercritical region is highlighted. Figure 2-12 illustrates a Temperature-Entropy diagram for $\mathrm{CO}_{2} \mathrm{I}$ which the critical point is located at the top of the dome. 
Gases are commonly referred to as behaving as an ideal or non-ideal (real) gas. Properties of ideal gases vary with only temperature whereas the properties of real gases vary with temperature and pressure. Real gas effects need to consider compressibility effects, specific heat capacity, and Van der Waals forces among other factors, depending on the fluid. It is important to understand that at supercritical state $\mathrm{CO}_{2}$ behaves as a real gas and compressibility effects are very influential and thus need to be accounted for in the design of the compressor.

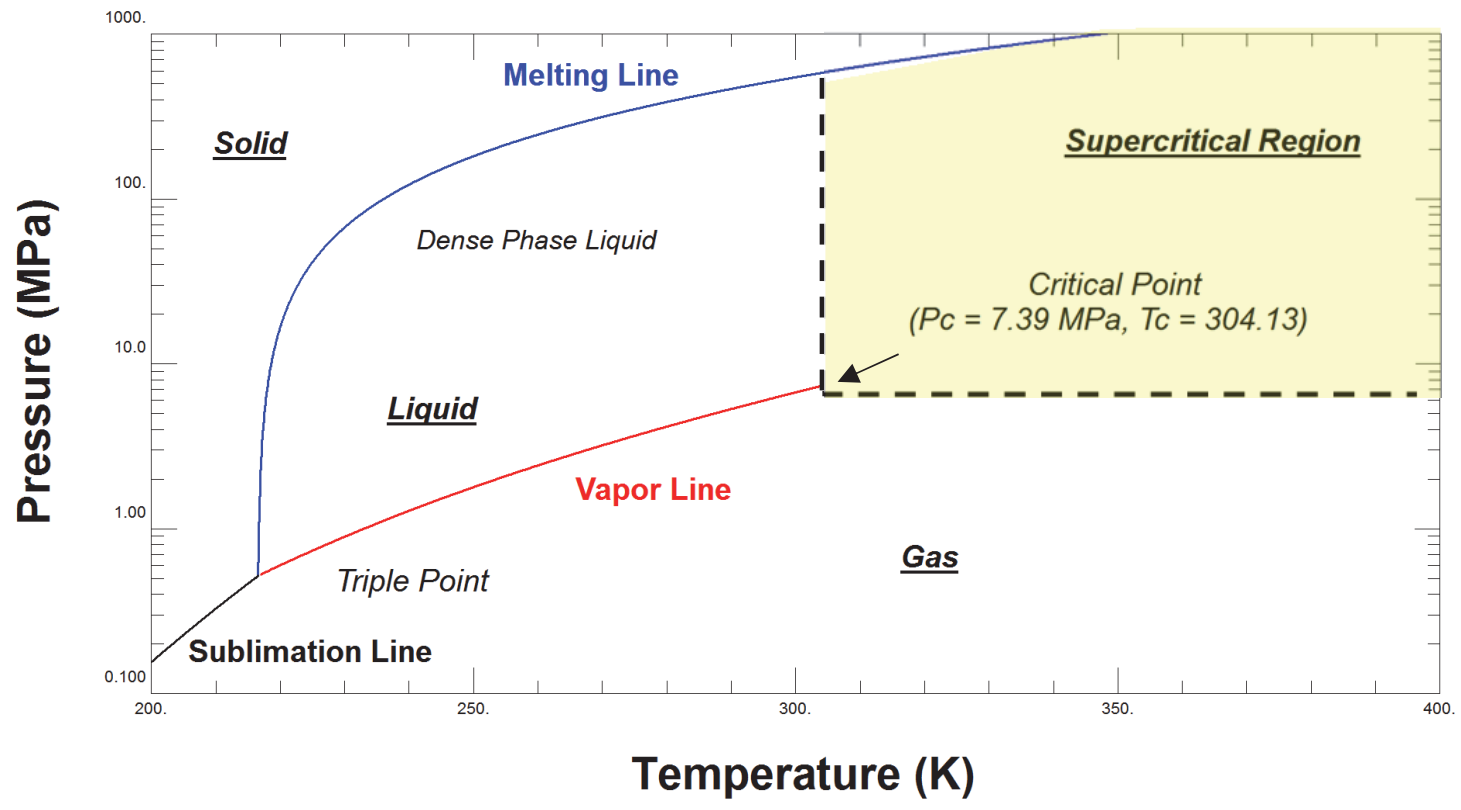

Figure 2-11: Phase diagram of $\mathrm{CO}_{2}$ (REFPROP, 2015). 


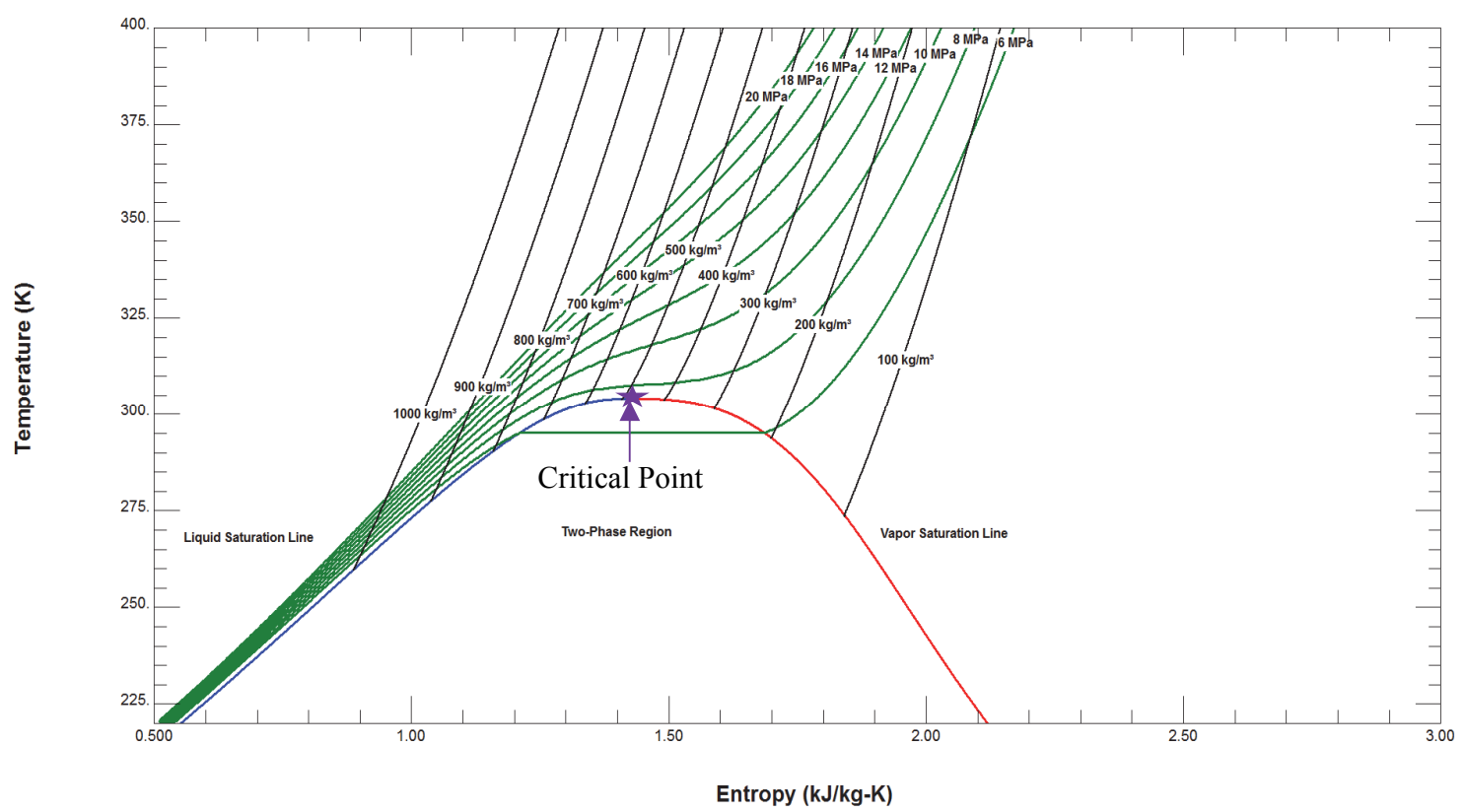

Figure 2-12:Temperature versus Entropy diagram of $\mathrm{CO}_{2}$ (REFPROP, 2015).

\subsubsection{Compressibility Factor}

The compressibility factor $(\mathrm{Z})$ is defined as the ratio between the actual specific volume of the fluid to the specific volume it would have if considered an ideal gas. The deviation from ideal gas is therefore described by the compressibility factor and introduced to the ideal gas equation as shown in Equation 2.11.

$$
\begin{aligned}
& Z=P v / R T \\
& Z=\frac{P}{\rho R T}
\end{aligned}
$$

The value of $\mathrm{Z}$ only provides the magnitude of the deviation, whereas the "gradient of $Z$ with respect to temperature and pressure determines the behavior of the fluid thermodynamic processes" (Baltadjiev, 2014). Ideal gases are defined by values close to unity; under such conditions compressibility effects are less influential and properties vary with temperature. As $\mathrm{Z}$ deviates from unity, the ideal gas assumptions 
break down and the fluid behaves like a real gas, in which properties vary with temperature and pressure. The compressibility Factor of $\mathrm{S}_{-} \mathrm{CO}_{2}$ at the critical point is $Z=29$.

Figure 2-13 illustrates the compressibility factor of several fluids. The figure illustrates the Principle of Corresponding States and shows that different fluids experience similar behavior as they approach the critical point. The fluid behavior is noticeable when the same coordinate axes are used (Moran and Shapiro, 2011). The same coordinate axes are achieved using the non-dimensional parameters of Reduced Pressure $P_{R}$ (Equation 2.12) and Reduced Temperature $T_{R}$ (Equation 2.13) (Moran and Shapiro, 2011).

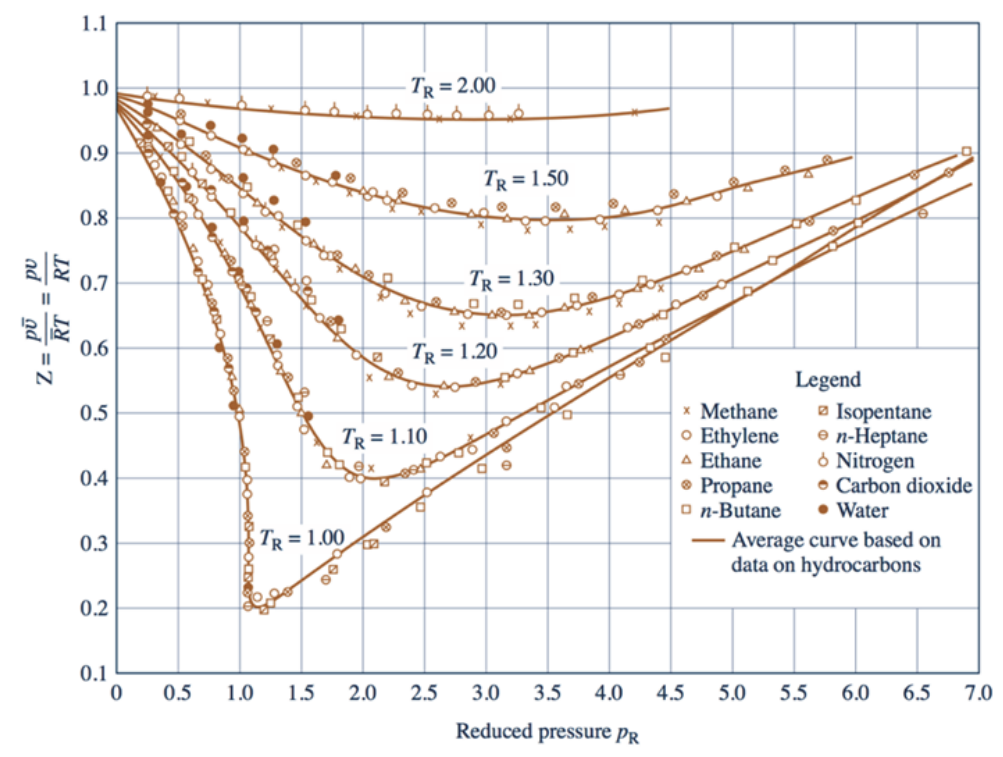

Figure 2-13: Generalize compressibility factor chart for different fluids (Moran and Shapiro, 2011).

$$
\begin{aligned}
& \text { Reduced Pressure }=\frac{P}{P_{c}} \\
& \text { Reduced Temperature }=\frac{T}{T_{c}}
\end{aligned}
$$


Figure 2-14 illustrate the compressibility factor of $\mathrm{CO}_{2}$ versus temperature for seven different isobars. Figure 2-15 highlights the approximate regions where the fluid is said to behave as ideal or real. Figure 2-15 illustrates the temperature range at which $\mathrm{Z}$ begins to sharply decrease. Notice that as the pressure approaches the critical pressure, the slope of the line sharply increases.

A low value of compressibility is beneficial to compressors as less energy is required to compress the fluid. Similarly, as demonstrated in the next section, the critical point experiences a density value similar to a liquid. Therefore, it can be said that as $\mathrm{Z}$ decreases, density increases as shown by Figure 2-16. It is important to understand that density is impacted by factors other than compressibility factor. As shown by the isobars, an increase in pressure also increases density, as one would expect.

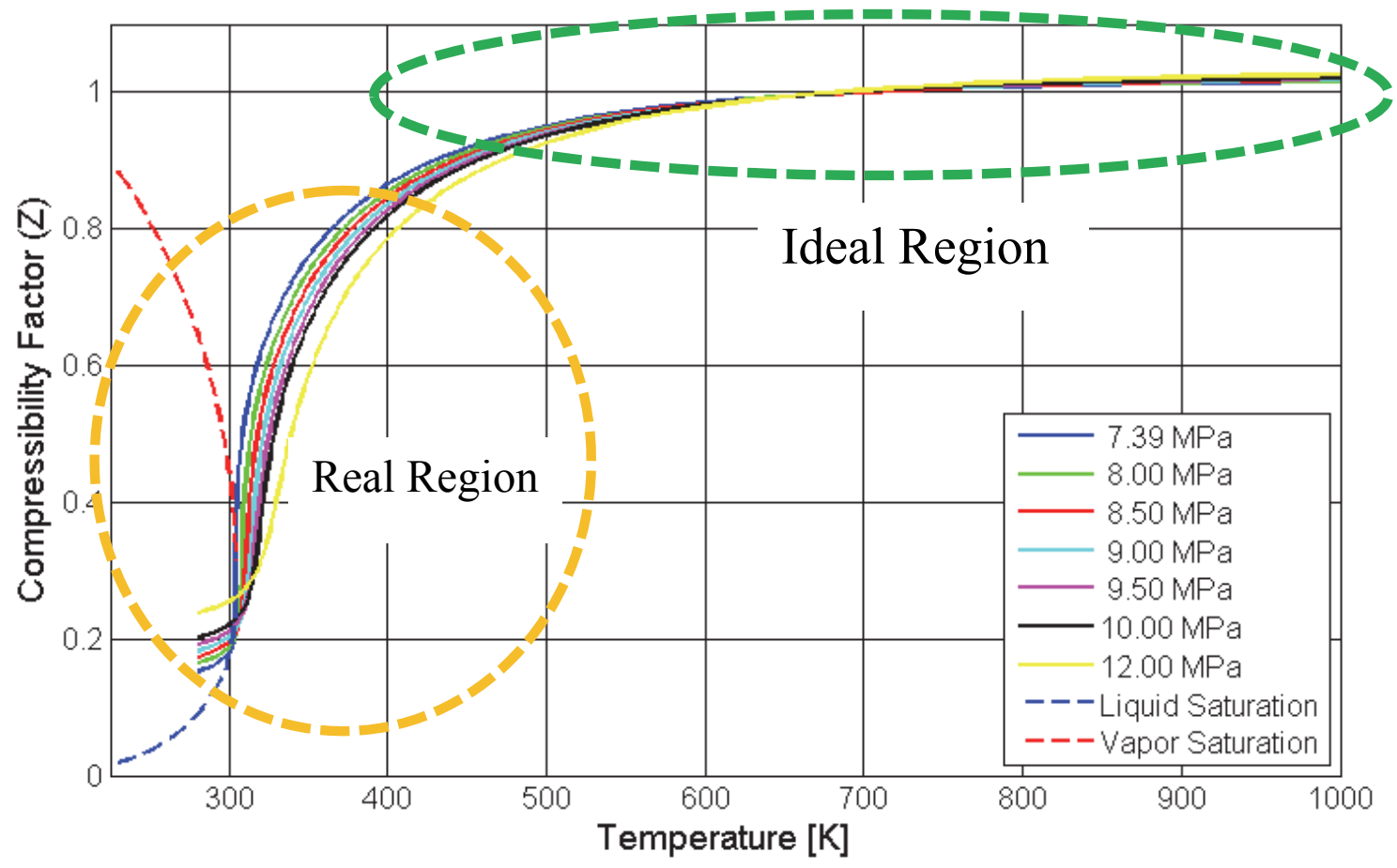

Figure 2-14: Compressibility factor (Z) of S- $\mathrm{CO}_{2}$ for a temperature range of $280 \mathrm{~K}$ to $1000 \mathrm{~K}$ (REFPROP, V9.1, 2015). 


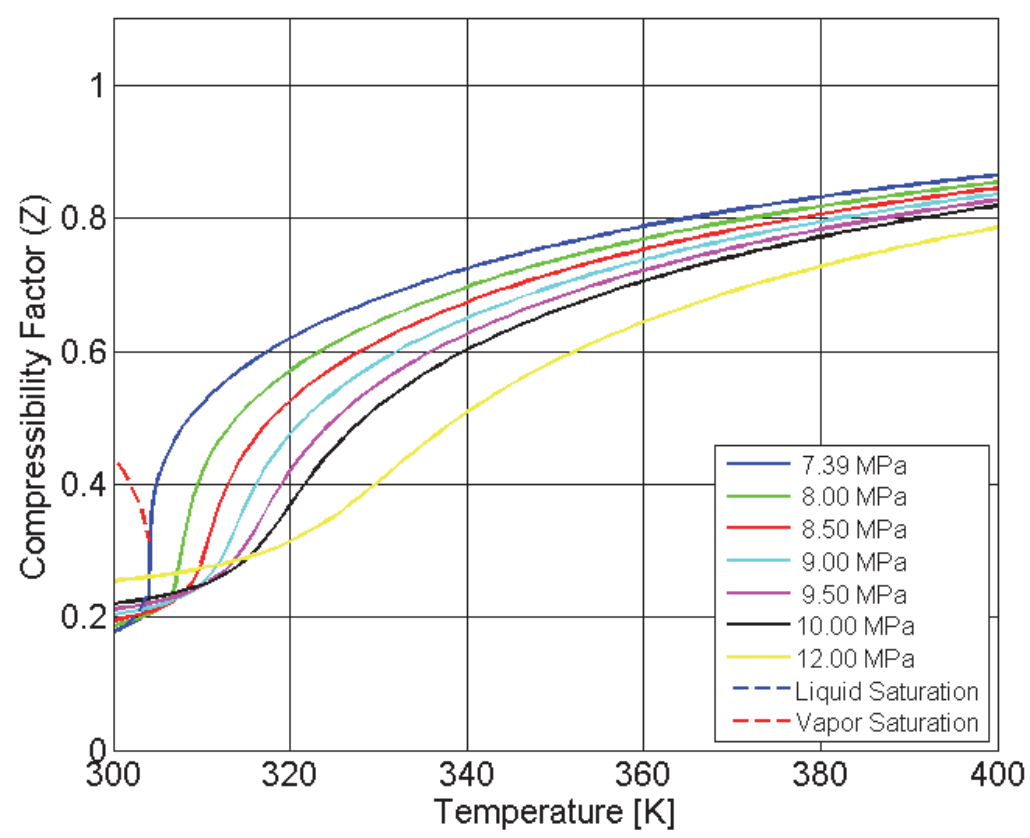

Figure 2-15: Compressibility factor $(\mathrm{Z})$ of $\mathrm{S}-\mathrm{CO}_{2}$ for a temperature range of $300 \mathrm{~K}$ to $400 \mathrm{~K}$ (REFPROP, 2015).

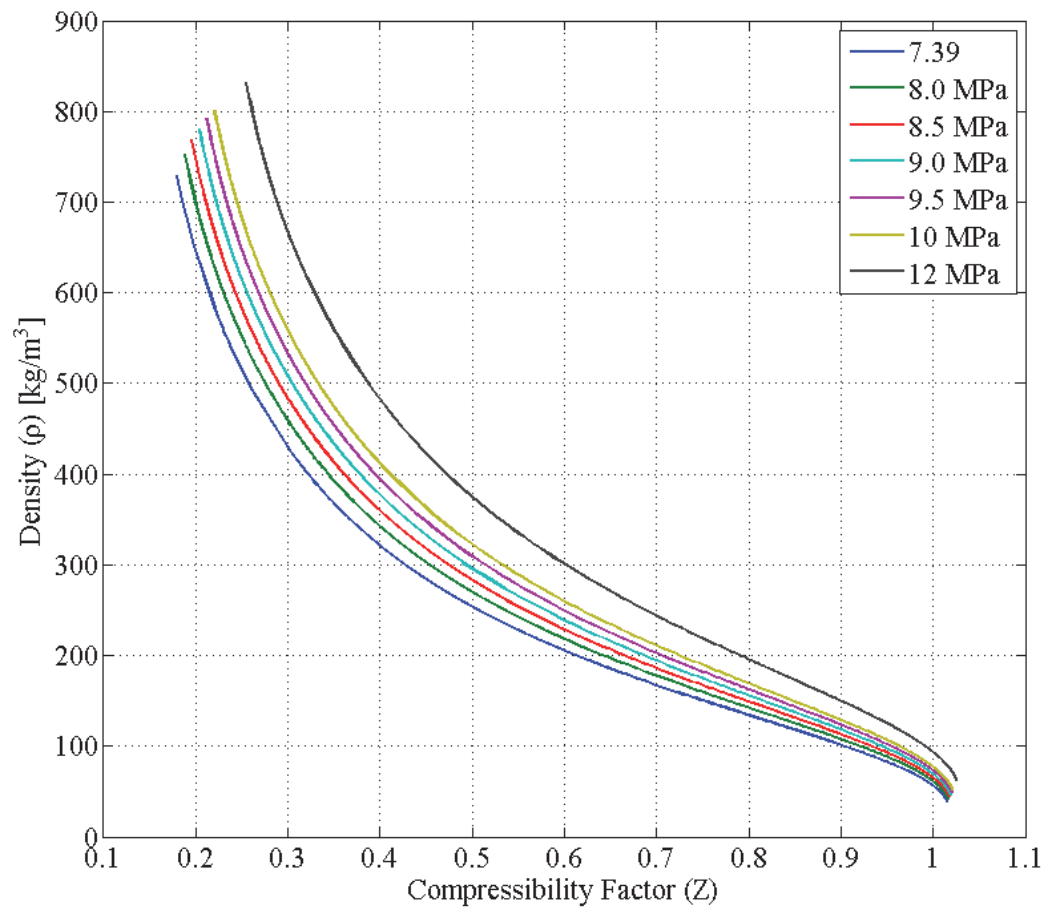

Figure 2-16: Description of the density values for different values of compressibility factor (REFPROP V9.1, 2015). 


\subsubsection{Properties of $\mathrm{S}-\mathrm{CO}_{2}$ near the Critical Point}

As the fluid approaches the critical point the properties are highly sensitive to the molecular interaction (Burk B, 1991). Therefore, small changes in temperature and pressure sharply affect the properties as shown in the next set of figures. At the critical point, the $\mathrm{S}-\mathrm{CO}_{2}$ experiences a large heat capacity, indicating that a vast amount of energy is required to increase the temperature. Figure 2-17 illustrates the heat capacity around the critical point for a given isobar at the critical pressure. The behavior of heat capacity as a function of temperature for different isobars is presented in Figure 2-18. The same figure illustrates how the heat capacity decreases with an increase in temperature and pressure. Each isobar has a specific point at which the maximum heat capacity is achieved, which is referred to as the pseudocritical point. These points must have a pressure and temperature greater than the critical. The black dashed line in Figure 2-18 illustrates the pseudocritical line. Understanding the effect of heat capacity is important as it relates to other fluid properties dependent on energy. 


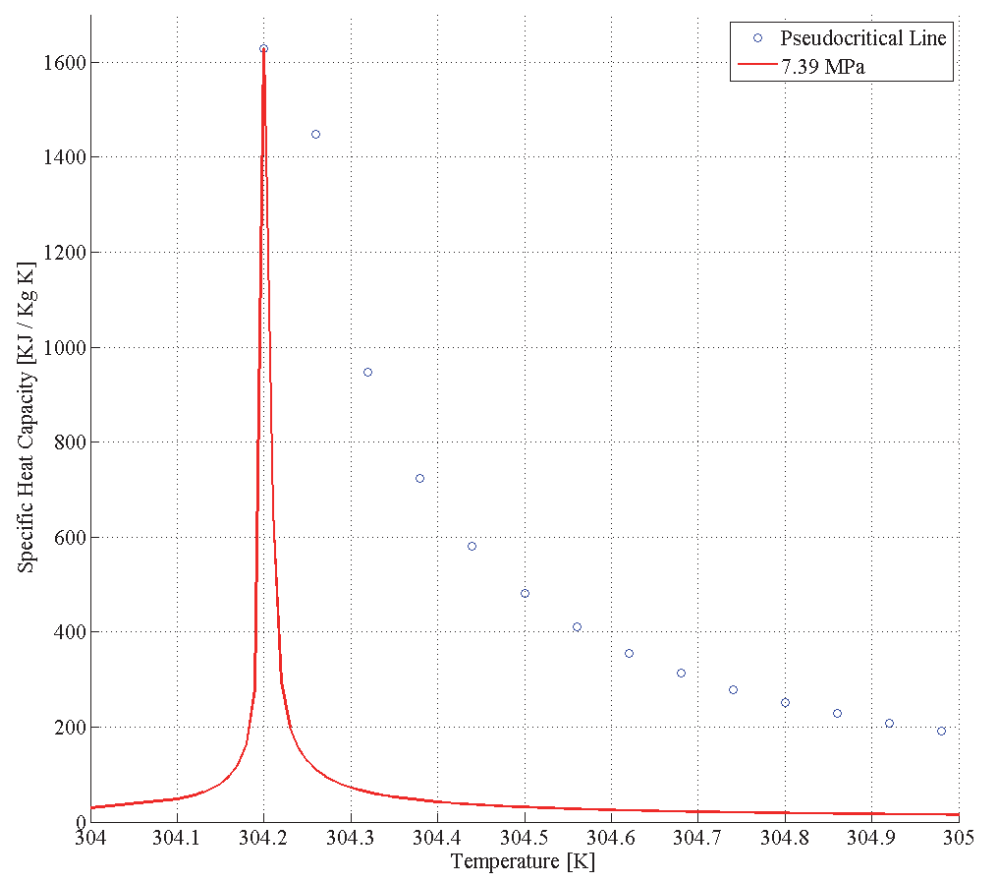

Figure 2-17: Plot of the constant specific heat $(\mathrm{Cp})$ near he critical pressure $(\mathrm{P}=7.39 \mathrm{MPa})$ and the corresponding pseudocritical points (REFPROP V9.1, 2015).

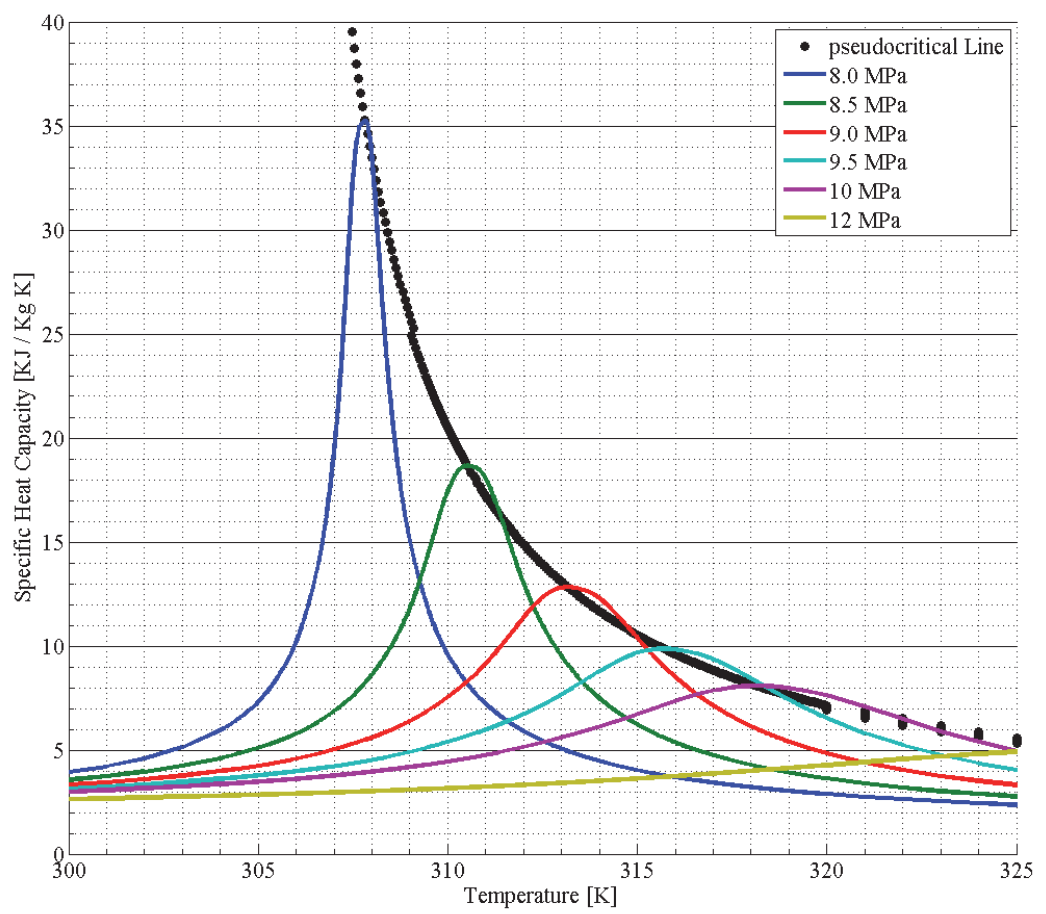

Figure 2-18: Plot of the constant specific heat $(\mathrm{Cp})$ at different isobars and the corresponding pseudocritical points (REFPROP V9.1, 2015). 
Figure 2-19 illustrates the density values for different isobars. It is possible to observe that as pressure increases the slope at which density changes also decreases. The change in slope highlights the strong relation between density and pressure near the critical point. The value of density as a function of pressure and temperature is mainly attributed to the molecular forces (attractive and repulsive) (Burk, 1991).

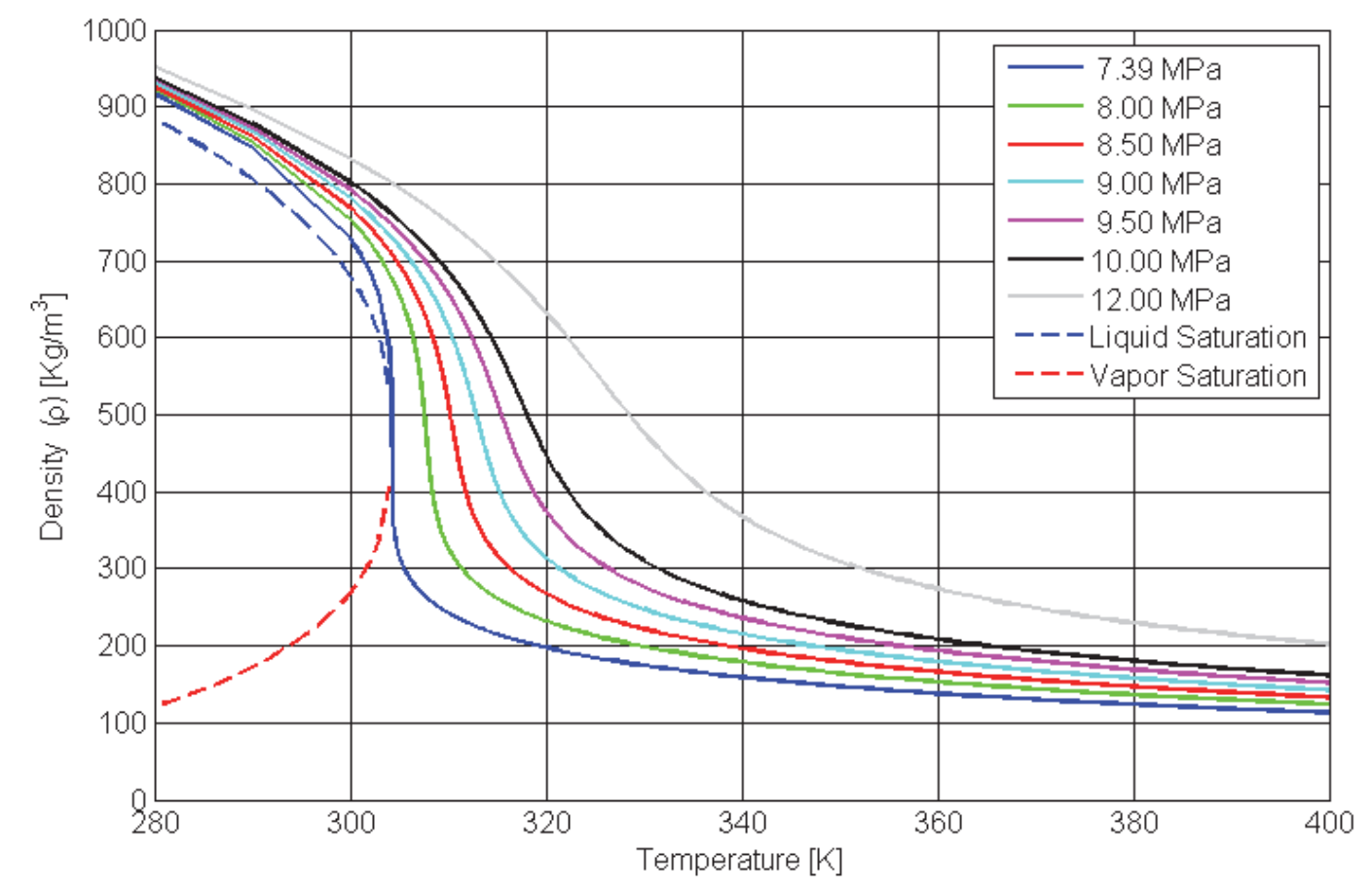

Figure 2-19: Density of $\mathrm{S}_{-} \mathrm{CO}_{2}$ for a temperature range of $280 \mathrm{~K}$ to $400 \mathrm{~K}$ (REFPROP V9.1, 2015).

The following set of figures (Figure 2-20 to Figure 2-22) are presented here to illustrate the changes in other properties of $\mathrm{CO}_{2}$ near the critical point. Properties like speed of sound and viscosity are important to consider in design of the compressor. 


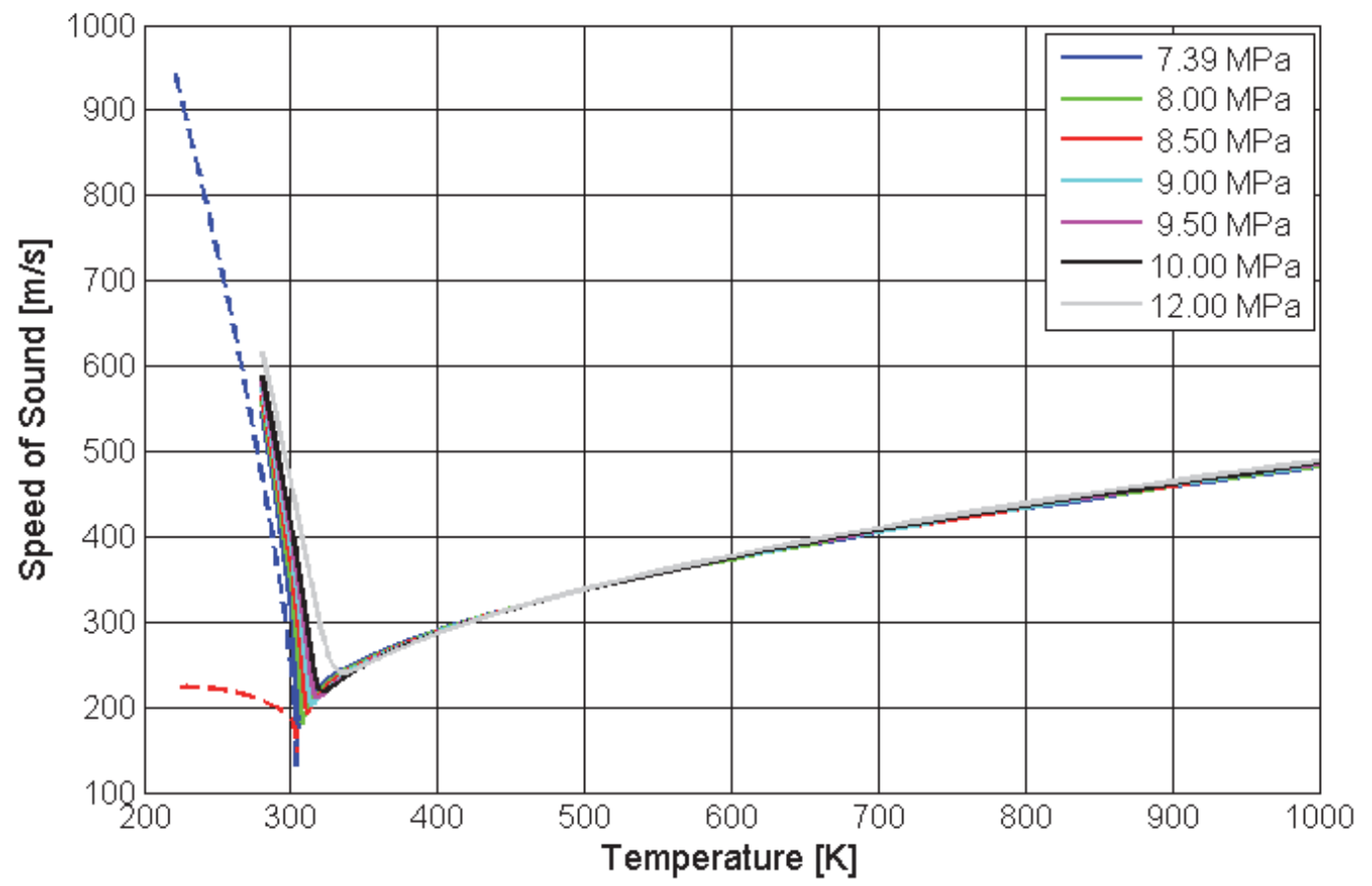

Figure 2-20: Speed of sound of S-CO 2 (REFPROP V9.1, 2015).

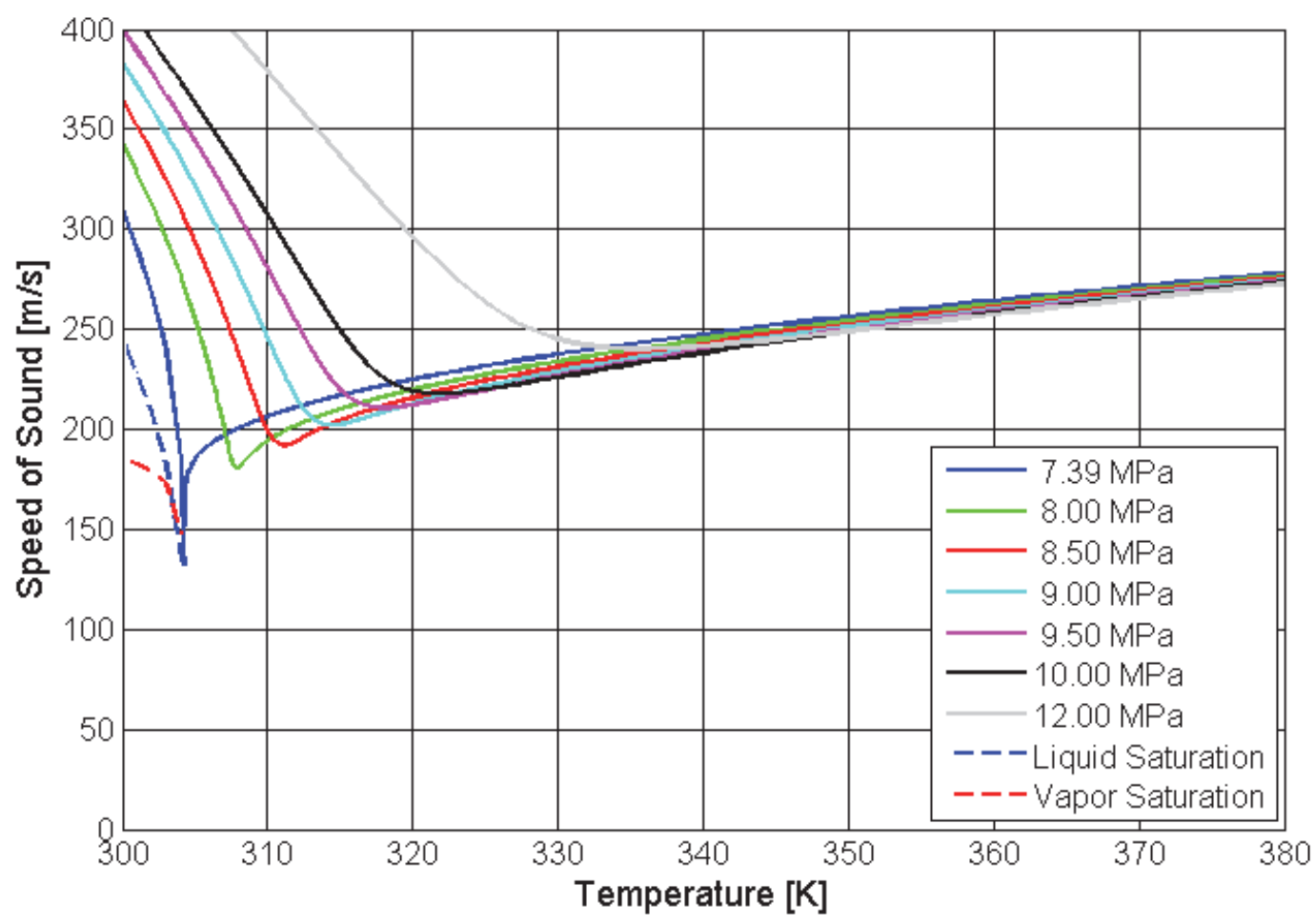

Figure 2-21: Speed of sound of $\mathrm{S}^{-\mathrm{CO}_{2}}$ for a temperature range of $300 \mathrm{~K}$ to $380 \mathrm{~K}$ (REFPROP V9.1, 2015). 

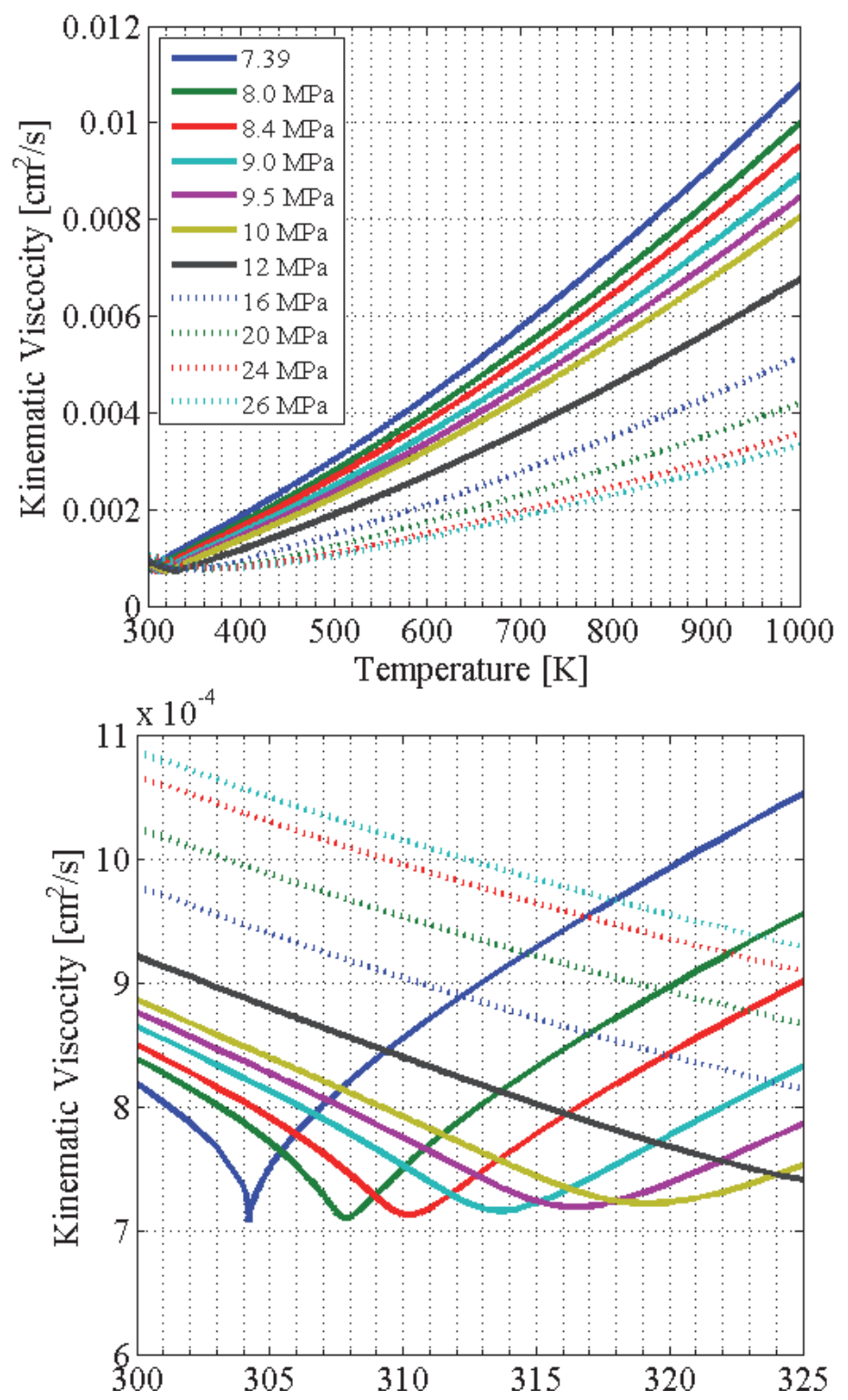

Figure 2-22: kinematic viscosity of S-CO $\mathrm{CO}_{2}(\mathrm{REFPROP}$ V9.1, 2015).

As shown by the previous figures and section, the properties near the critical point are highly dependent on pressure and temperature. Therefore, as the fluid deviates from ideal behavior, a proper Equation of State is required to accurately determine the properties. In this investigation, the software REFPROP V9.1 was used. REFPROP is a reference fluid thermodynamic and transport property database developed by US National Institute of Standards and Technology (NIST). For $\mathrm{CO}_{2}$, REFPROP uses the 
Span \& Wagner (1996) equation of state. REFPROP allows the user to obtain some of the most accurate values for $\mathrm{S}-\mathrm{CO}_{2}$ properties.

However, the 3D aerodynamic analysis use the CFX built-in Peng Robinson EOS as, as the flow behaves an ideal gas (as explained in Chapter 3). When the flow is well within the ideal region, the use of the Peng Robinson equation is acceptable. However, when applying the Scaling Methodology and Projection Methodology, as it will be shown in later chapters, it is fundamental that the proper EOS be used.

\subsubsection{Isentropic Exponent}

From the isentropic relations of ideal gases, the isentropic process is defined by Equation 2.15. This assumes that gamma $(\gamma)$ is constant. Gamma $(\gamma)$ is defined as the ratio of the specific heat at constant pressure to the specific heat at constant volume as and is shown in Equation 2.16.

$$
\begin{aligned}
& \frac{P}{\rho^{\gamma}}=\text { Const } \\
& \gamma=\frac{C p}{C_{v}}
\end{aligned}
$$

Figure 2-23 \& Figure 2-24 illustrates the values of gamma $(\gamma)$ for $\mathrm{S}-\mathrm{CO}_{2}$. It is possible to observe that gamma is relatively constant until it approaches the critical point, as expected due to the dependence on $\mathrm{Cp}$. The dependence on $\mathrm{Cv}$ is smaller as $\mathrm{Cv}$ experiences lower changes. 


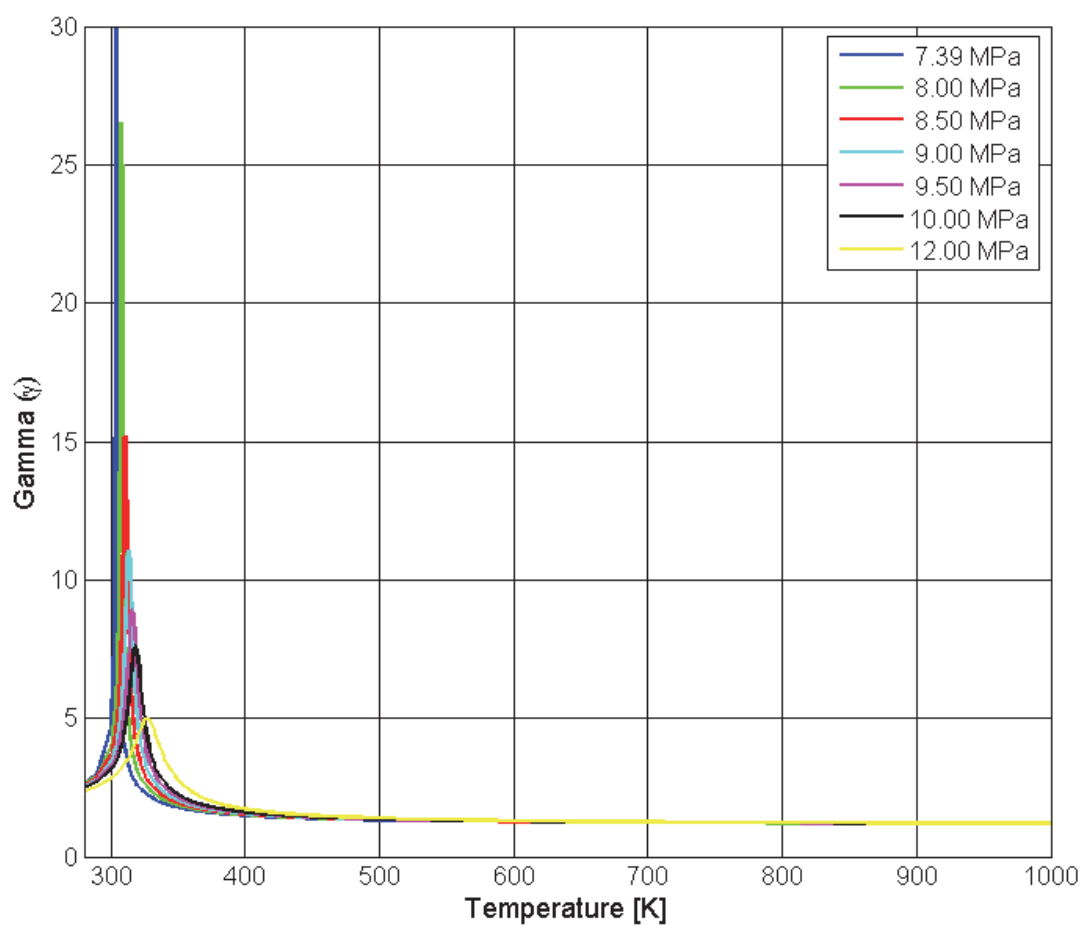

Figure 2-23:Gamma variation for $\mathrm{S}-\mathrm{CO}_{2}$.

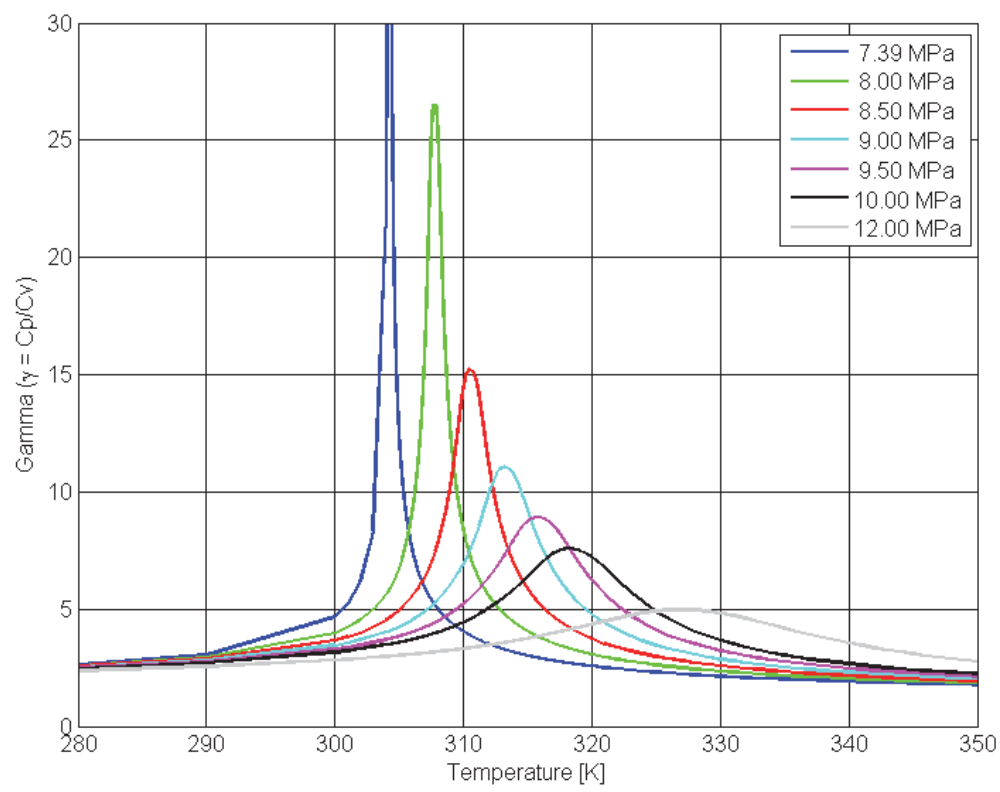

Figure 2-24: Close-up of gamma for $\mathrm{S}-\mathrm{CO}_{2}$

A similar equation to Equation 2.15 can be applied for non-ideal gases. The comparable equation makes use of the isentropic exponent $\left(\mathrm{n}_{\mathrm{s}}\right)$. Here, gamma is 
substituted by the isentropic exponent $\left(\mathrm{n}_{\mathrm{s}}\right)$ as seen in Equation 2.17 (Baltadijiev, 2012). However, it is important to understand that this substitution assumes constant value for $\mathrm{n}_{\mathrm{s}}$.

$$
\frac{P}{\rho^{n_{s}}}=\text { Const }
$$

The isentropic exponent can be derived by expressing the isentrope in differential form and substituting $\gamma$ for $\mathrm{n}_{\mathrm{s}}$, Equation 2.18 defines the isentrope in differential form. A complete derivation of the isentropic exponent is presented in (Baltadijiev, 2012).

$$
\left.\frac{d p}{p}+n_{s} \frac{d v}{v}\right\rceil_{s=\text { Const }}
$$

Equation 2.18 can then be rearranged to yield the isentropic exponent $n_{s}$ (Baltadijiev, 2012)

$$
n_{s}=-\frac{v}{p}\left(\frac{\partial p}{\partial v}\right)_{s}
$$

The isentropic exponent can also be expressed as shown in Equation 2-20 (Baltadijiev, 2012).

$$
n_{s}=\frac{\gamma Z}{\left(z-P\left(\frac{\partial Z}{\partial p}\right)_{T}\right)}
$$

\subsubsection{Speed of Sound}

The speed of sound is derived from the equation of motion. It is assumed that the compression from a weak sound wave is an isentropic process as shown by Equation 2.21

$$
a=\left(\frac{\partial P}{\partial \rho}\right)_{s}
$$


Subsequently, the speed of sound can be expressed in terms of the isentropic exponent and the modified equation of state as shown by Equation 2.22. Where isentropic exponent $\left(\mathrm{n}_{\mathrm{s}}\right)$, compressibility factor $(\mathrm{Z})$, temperature $(\mathrm{T})$ and the gas constant $(\mathrm{R})$ and the gas constant for carbon dioxide is $188.9 \frac{\mathrm{J}}{\mathrm{Kg} \mathrm{K}}$.

$$
a=\sqrt{n_{s} Z R T}
$$

\subsubsection{Dimensional Analysis and Non-Dimensional Parameters for Non-Ideal Compressible Fluids}

Throughout Chapter 2, the importance of real flow behavior and the changes in properties have been presented. The compressibility factor and its importance in describing the magnitude of the deviation of a real gas from ideal conditions was introduced in Section 2.10.1. The importance of the isentropic exponent was introduced in Section 2.10.3. These parameters are of great importance to dimensional analysis of real compressible gases.

Chapter 4 and 7 will present two different methodologies used during the design process of this centrifugal compressor. The methodologies use the non-dimensional parameters presented below.

Dimensional Analysis is a powerful approach used to help reduce and express the dimensional variables of a fluid problem in terms of a smaller number of nondimensional parameters. The process of determining non-dimensional parameters allows the user to define the necessary parameters needed to achieve dynamic similarity. 
Dynamic similarity is completely achieved between two flows, or components, when all the non-dimensional parameters (ratios) are the same for both flows. The Buckingham $\Pi$ Theorem is commonly employed to help identify the number of independent nondimensional ratios needed to describe the problem of interest. The Buckingham ПTheorem procedure is extensively covered in introductory fluid dynamics textbooks such as White (2010). Similarly, common non-dimensional parameters for ideal compressible fluids can be obtained from turbomachinery textbooks such as Dixon (2014) or Japikse \& Baines (1997).

From Sjolander (2017), it is possible to define the compressible flow of non-ideal gases in terms of nine independent variables $(\mathrm{M})$.

Independent Variables: $D, N, \dot{m}, T_{01}, \rho_{01}, \mu, Z, R, n_{s}$

There are four basic dimensions $(\mathrm{N})$. The Buckingham $\Pi$-Theorem states that M$\mathrm{N}$ equal the total number of criteria of similarity. Therefore, there are five criteria of similarity parameters as shown below. The derivation of the parameters is shown in Sjolander (2017).

1. Speed Coefficient

2. Flow Coefficient

$$
\Pi_{\mathrm{N}}=\frac{N}{\sqrt{T_{o 1}}} \frac{D}{\sqrt{Z R n_{s}}}
$$

3. Reynolds Number

$$
\Pi_{\mathrm{m}}=\frac{\dot{m} \sqrt{T_{o 1}}}{P_{o 1}} \sqrt{\frac{Z R}{n_{s}}} \frac{1}{D^{2}}
$$

4. Compressibility Factor

\section{Isentropic Exponent}

$$
R e=\frac{\rho_{01} N D^{2}}{\mu}
$$

$$
\text { Z }
$$

$\mathrm{n}_{\mathrm{s}}$

For this investigation, partial similarity was used to determine the partially similar operating design point, as it will be described in later chapters. The partial similarity was 
achieved by marinating the Flow $\left(\Pi_{\mathrm{m}}\right)$ and Speed $\left(\Pi_{\mathrm{N}}\right)$ coefficient constant. Due to a geometry constraint, Reynolds Number was not matched. However, its effects on turbomachinery are well understood and were accounted for by using a correction formula as shown by Equation 2.26 and as presented by Sjolander, (2017).

$$
\frac{1-\eta_{P}}{1-\eta_{M}}=\left(\frac{R e_{M}}{R e_{P}}\right)^{n}
$$

where:

$$
\begin{array}{ll}
\mathrm{n}=0.1 \text { for centrifugal compressor } & \mathrm{M}-\text { Model } \\
\mathrm{n}=0.2 \text { for axial compressors } & \mathrm{P} \text { - Prototype }
\end{array}
$$

In this investigation, the isentropic Exponent $\left(\mathrm{n}_{\mathrm{s}}\right)$ and Compressibility Factor $(\mathrm{Z})$ were not matched. As expected, in a compression process near the critical region these variables experience sharp changes between inlet and outlet, and further studies to understand their effects are required. The changes experienced by $\mathrm{n}_{\mathrm{s}}$ and $\mathrm{Z}$ are further discussed in later chapters.

\subsection{Chapter Summary}

Chapter 2 covered several fundamental areas towards the proper understanding of this thesis.

- Section 2.2 to Section 2.6 provide background information to $\mathrm{S}-\mathrm{CO}_{2}$ Brayton cycles and waste heat recovery systems.

- The subsequent Sections 2.7 to Section 2.10 presented a literature review of popular compressor design methodologies and suggested guidelines and values that designers can follow. This section and the information presented are strictly 
based on common turbomachinery theory which is based on ideal gas values, most commonly air.

- Section 2.11, highlighted the importance of treating supercritical carbon dioxide as a real fluid and the influence the critical point has on the fluid properties.

- The last area of interest, Section 2.12 presented the non-dimensional parameters used to describe the turbomachinery flows of compressible non-ideal gases. 


\section{Chapter 3}

\section{Design Process}

\subsection{Introduction}

Designing a compressor is challenging in nature. Designing centrifugal compressors for $\mathrm{S}-\mathrm{CO}_{2}$ adds a level of complexity due to the reasons presented in Chapter 1 and Chapter 2. Due to the nature of the fluid behaving as a real gas, being at a supercritical state, and near the critical point, the 3D aerodynamic analysis in CFD encountered challenges in achieving convergence. Because of the time constraints of the project, non-dimensional parameters were used to overcome the convergence challenges and complete the 3D aerodynamic study and obtain the compressor performance maps. The use of non-dimensional parameters influenced the design process of the compressor, and hence, a modified design process was followed. This chapter aims to provide a clear road map of the design process of this compressor, and most importantly, provide a proper connection between the design process, aerodynamic analysis, the use of nondimensional parameters, and the creation of the compressor characteristic maps.

\subsection{Actual Design Process}

The normal design process of a centrifugal compressor is illustrated in Figure 3-1. It is possible to observe that the design process is linear and iterative. Similarly, it is possible to observe how the design point was obtained from the cycle thermodynamic analysis as described by the Gas Turbine Design Process in Chapter 2. 


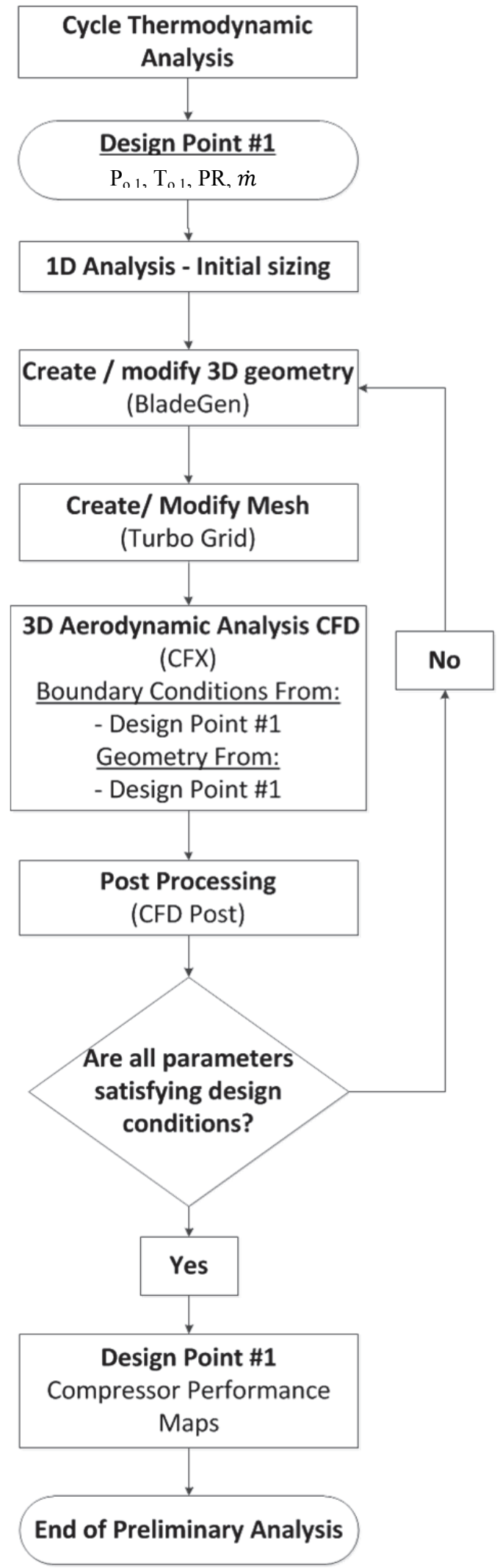

Figure 3-1: Common design process of a centrifugal compressor. 
Recall that the CFD encounters convergence challenges near the critical point. Therefore, non-dimensional parameters were used to define a partially similar operating point (Design Point \#2) in a region where the CFD could achieve proper convergence. The aerodynamic analysis was completed in this region and then, through similarity, the performance map of the machine can be approximated at the desired design point (Design Point \#1) by means of the Projection Methodology.

Chapter 2 introduced the concept of dimensional analysis, and the independent nondimensional parameters for non-ideal compressible gasses. Two different methodologies that use these parameters were applied in this project. The first is referred to as the Scaling Methodology and the second is referred to as the Projection Methodology. The first methodology is presented in Chapter 4, and the second is presented in Chapter 7.

The objective of this chapter is to present the methodologies and outline present a clear road map of when the methodologies were used throughout the design process as well as to clarify the process shown in Figure 3-2. Furthermore, the figure highlights where the two methodologies were incorporated.

The main difference between the Typical Design Process (Figure 3-1) and the Actual Design Process (Figure 3-2) is that there are two design points, \#1 and \#2. It is important to understand that the design process of this investigation completed all the steps shown in Figure 3-2. Design point \#1 contributed towards the initial sizing and dimensions for the initial geometry. Design point \#2 corresponds to the partially dynamically operating point, and it provides the information needed by the Boundary Conditions in the CFD. 


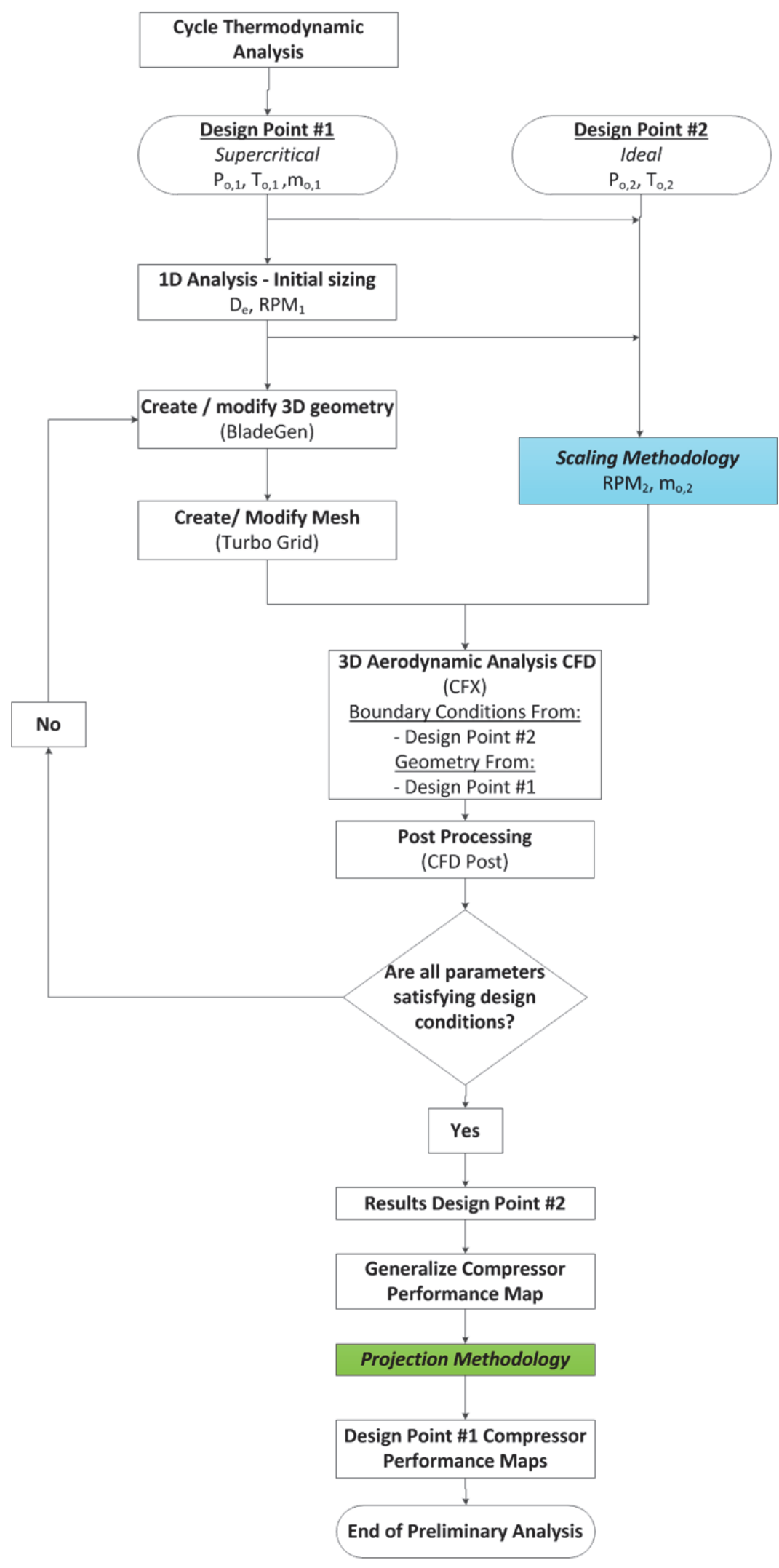

Figure 3-2: Modified design process, with the use of dimensional analysis and different Methodologies. Scaling methodology is highlighted in blue and the projection methodology is highlighted in green. 


\subsection{Design Point \#1 versus Design Point \#2}

The main difference between the two design points is the region where they operate. Design Point \#1 corresponds to the Actual Design Point of interest, near the critical region and behaves as a real gas. On the other hand, Design Point \#2 is a partially dynamically similar operating point and is used as the Boundary Conditions for the CFD Modelling. Design Point \#2, operates within the supercritical regime but it is far away from the critical point allowing the use of ideal gas law assumptions as shown in Figure 3-3. Being in the ideal gas region the modelling is simplified and CFD can achieve proper convergence.

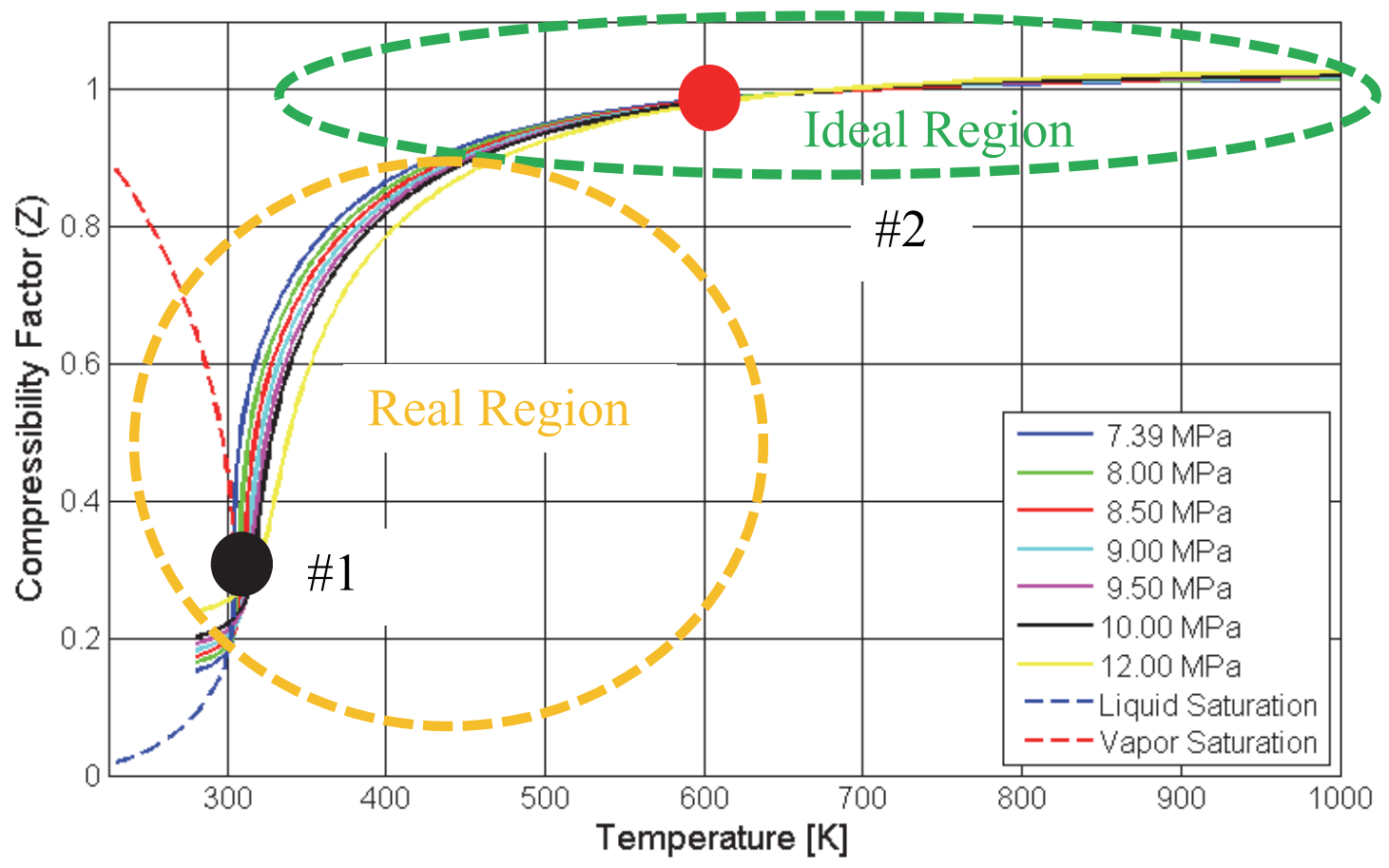

Figure 3-3: Compressibility factor of S- $\mathrm{CO}_{2}$. Orange circle highlights the area of real gas behaviour. Black circle indicates the location of Design Point \#1. The green ellipse highlights the area of ideal gas behavior. The red circle indicates the location of Design Point \#2.

The Scaling Methodology (Chapter 4) is the link between Design Point \#1 and Design Point \#2. By applying the independent non-dimensional parameters, it is possible 
to determine the mass flow rate $(\dot{m})$ and RPM required at Design Point \#2 based on the desired actual design point (Design Point \#1). These values provide a partially dynamically similar operating point and are used as the boundary conditions in CFD. Therefore, the 3D aerodynamic results presented in Chapter 6 correspond to Design Point \#2. Figure 3-4 illustrates the location of the two design points in a Temperature versus Entropy diagram.

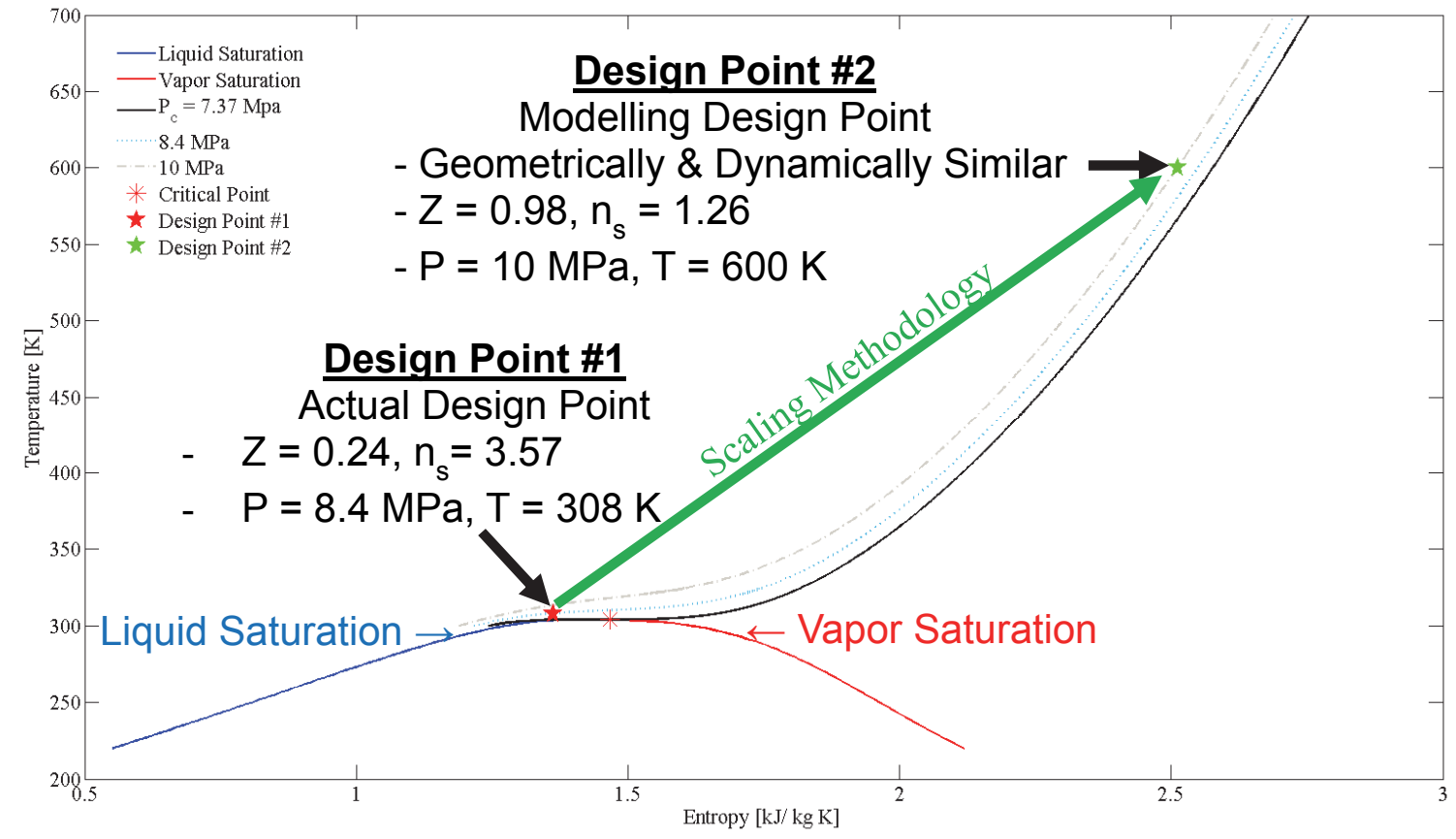

Figure 3-4: Illustration of the Actual Design Point (Design Point \#1) and Modelling Design Point (Design Point \#2) which is the partially dynamically operating point.

The results from the CFD (Chapter 6) allow the calculation of the dependent nondimensional parameter, Enthalpy Rise $\left(\Pi_{h}\right)$. This parameter is important as it allows for the creation of the Generalized Compressor Map.

The Projection Methodology (Chapter 7), is the link between the Generalized Compressor Map at Design Point \#2 and the expected compressor maps of Design Point 
\#1. This methodology uses the independent and dependent non-dimensional parameters. From the operating points in the Generalized Compressor Map, the user can calculate the corresponding points of Design Point \#1. Therefore, through similarity, it is possible to derive the expected performance map corresponding to the desired Design Point. The Generalized Compressor Map becomes an important tool as one can derive the compressor map of any other desired operating point as it is shown in Chapter 7.

The final results of this study then provide the preliminary sizing of the component, an understanding of the aerodynamic flow and the estimated preliminary compressor performance maps of Design Point \#1.

\subsection{Chapter Summary}

As mentioned, the objective to this chapter is to present a clear outline of the design process. The chapters of this thesis will follow the design process as shown in Figure 3-5.

- Chapter 4 presents the Design Point \#1, the initial sizing, and introduces the Scaling Methodology.

- Chapter 5 presents the different software packages from ANSYS Workbench that were used in this project.

- Chapter 6 present the results of the 3D aerodynamic analysis, and compare the initial geometry to the final preliminary geometry.

- Chapter 7 introduces the Enthalpy Rise Coefficient $\left(\Pi_{\mathrm{h}}\right)$, the Projection Methodology as well as the final compressor performance maps corresponding to Design Point \#1. 


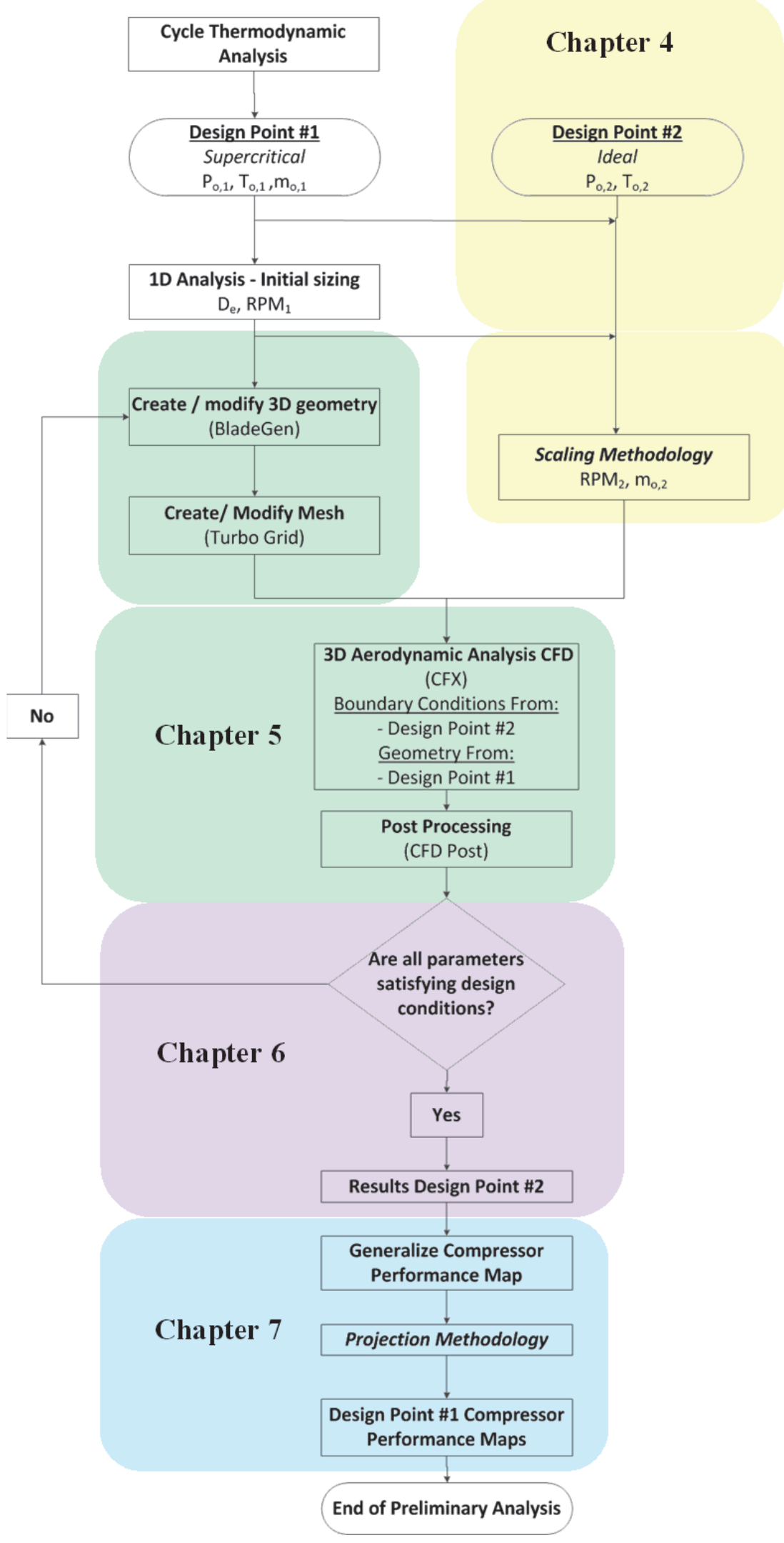

Figure 3-5: Design procedure and the content to each chapter in this thesis. 


\section{Chapter 4}

\section{Design Point, Initial Sizing and Scaling Methodology}

\subsection{Introduction}

This chapter introduces the design point conditions corresponding to Design Point \#1. Section 4.2 presents Design Point \#1 and describes an important trend in density. Section 4.2.1 discusses condensation. Section 4.3 Provides an overview of the initial sizing. Section 4.4 Introduces the Scaling Methodology and provides a summary of the operating conditions of Design Point \#2.

\subsection{Design Point}

The design point was chosen following a collaborative steady state thermodynamic analysis between Carleton University and the industrial partner. The thermodynamic analysis compared several cycles for waste heat recovery. Under this consideration, Design Point \#1 was chosen as the compressor inlet operating point, with values shown Table 4-1.

Table 4-1: Compressor inlet conditions for a $10 \mathrm{MW}_{\mathrm{e}}$ waste heat recovery $\mathrm{S}-\mathrm{CO}_{2}$ Brayton Power Cycle. These conditions correspond to Design Point \#1.

\begin{tabular}{|c|c|c|}
\hline \multicolumn{3}{|c|}{ Design Point \#1 } \\
\hline Parameters & Units & Value \\
\hline $\mathbf{P}_{0,1}$ & {$[\mathrm{MPa}]$} & 8.4 \\
$\mathrm{~T}_{0,1}$ & {$[\mathrm{~K}]$} & 308.15 \\
\hline $\mathbf{Z}_{0,1}$ & - & 0.24 \\
\hline Pressure Ratio & - & 2.84 \\
\hline Mass Flow & {$[\mathrm{kg} / \mathrm{s}]$} & 170 \\
\hline Rotational Speed & {$[\mathrm{Rev} / \mathrm{min}]$} & 21,000 \\
\hline
\end{tabular}


Notice that Design Point \#1 is located at a pressure and temperature higher than the critical point $\left(\mathrm{T}_{\mathrm{c}}=304.13, \mathrm{P}_{\mathrm{c}}=7.39 \mathrm{MPa}(\mathrm{REFPROP}, 2015)\right)$. This point was chosen to minimize the probability of condensation at the compressor inlet, which is further explained in Section 4.2.1. It is commonly mentioned in the $\mathrm{S}-\mathrm{CO}_{2}$ literature that the best compressor performance is achieved when the inlet conditions are close to the critical point. However, it is important to understand the physics of the compressor near the critical point. The benefits of compressing in this region are attributed to the density values near the critical point (as discussed in Section 2.10.2). As shown in Figure 4-1, it is possible to observe that when temperature and pressure increase simultaneously the density experiences relatively small changes. This trend is highlighted in Figure 4-1 by the solid black arrow and the red and blue dots.

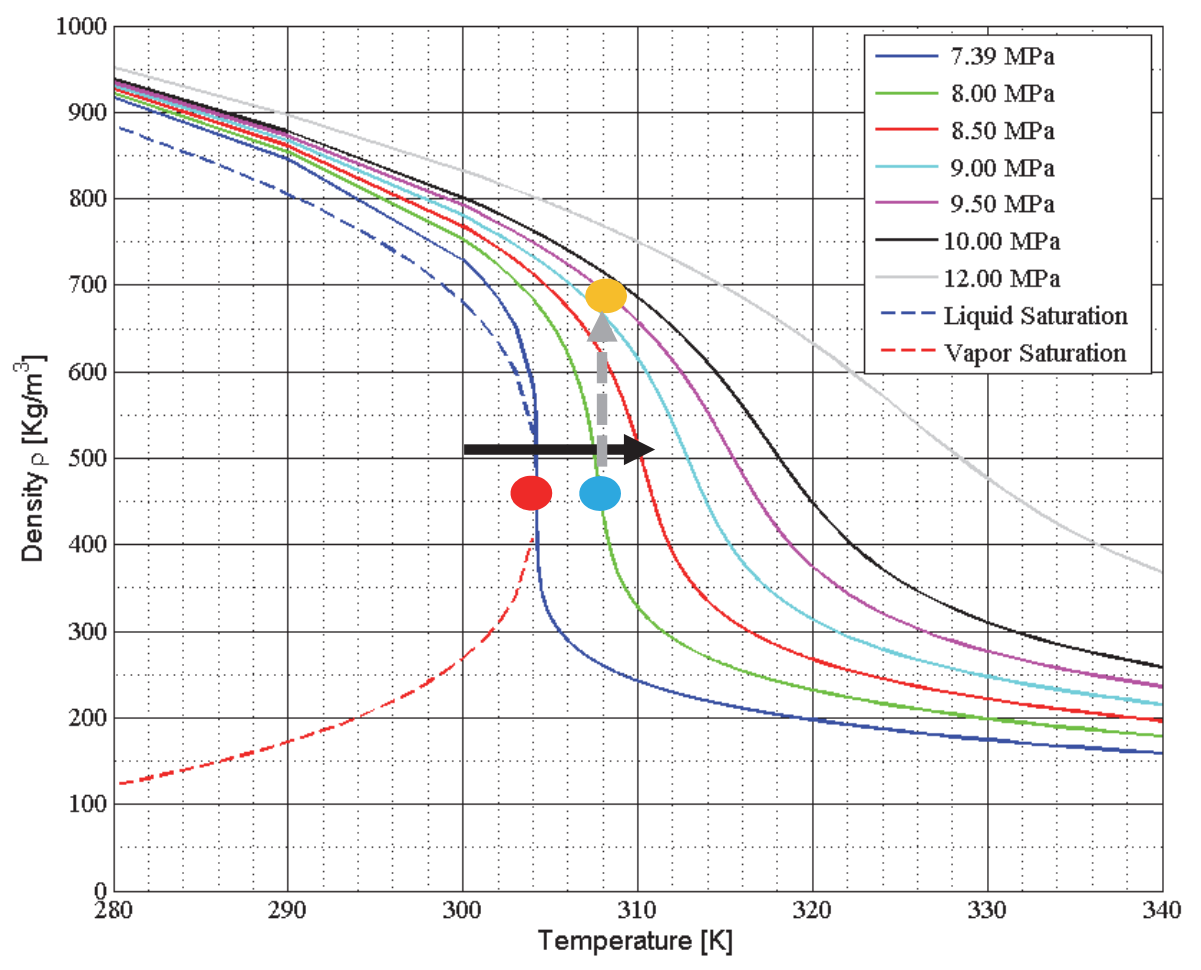

Figure 4-1: Density of S- $\mathrm{CO}_{2}$ for a temperature range of $280 \mathrm{k}$ to $340 \mathrm{~K}$. 
For the following discussion, values are chosen arbitrarily. Taking the critical point (red dot), from Figure $4-1$ it is possible to read a density of $\sim 460 \mathrm{~kg} / \mathrm{m}^{3}$. If one increases the temperature to $308 \mathrm{~K}$ and increase the pressure to $8 \mathrm{MPa}$ (blue dot), one can see that the density remains nearly constant at $\sim 460 \mathrm{~kg} / \mathrm{m}^{3}$. It is very important to understand that this example only relates to the inlet conditions of the machine. The flow inside the compressor will experience density changes as it is a compressible fluid.

This trend in density allows designers to change the inlet conditions to maintain a similar performance as the one achieved near the critical point. It can be highly valuable to the designer in cases when the cycle/component need to operate at off-design (waste heat recovery units may experience large heat source variations as they are dependent on the main cycle and its operation). Assuming the inventory control system and the system itself can handle significant pressure variations, then the plant operators can shift the inlet pressure to better accommodate the inlet temperatures and achieve a similar density to that of the design point. This can be beneficial to the system as it can maintain a constant power output for a fixed rotational speed. It is important to mention that inlet temperatures are highly dependent on the available cooling and ambient temperatures. Therefore, in hot, dry regions, hotter inlet temperatures may be unavoidable. In these regions, if an inlet pressure near the critical point was be selected then the density would be lower due to the higher temperatures, as in Figure 4-1.

In some cases, depending on the selected inlet temperature, a higher density can be obtained by increasing the pressure alone (as shown by the dotted grey arrow and the blue and orange dots), therefore, requiring less energy to achieve the same compression requirement for the same pressure rise. For example, given the blue dot $(308 \mathrm{~K}$ and 8 
$\mathrm{MPa}$ ), if temperature is held constant and pressure can be increased to $9 \mathrm{MPa}$ (Orange dot), there it is an observed density increased from $\sim 460 \mathrm{~kg} / \mathrm{m}^{3}$ to about $\sim 630 \mathrm{~kg} / \mathrm{m}^{3}$. The expected preliminary performance map of this operating point is presented in chapter 7 .

The inlet conditions are important to the compressor performance not only at the design point, but also at off-design. As will be discussed in Chapter 7, due to the nature of $\mathrm{S}-\mathrm{CO}_{2}$, several compressor performance maps are required to completely express the compressor's operating region at the design point and at off-design. Understanding the effects of the inlet temperature and pressure can allow the plant operator to maximize the plant performance and determine the optimal operating condition while at off design. Similarly, the thermodynamic analysis provided insights regarding the potential offdesign points. Therefore, this information needs to be considered by the designer while sizing and designing the compressor. A well-designed compressor would allow a wide operating range and satisfy the specified Design Point $\# 1$ and the required off-design points.

\subsubsection{Two-phase Region and Condensation}

The main reason why inlet conditions are commonly shifted away from the critical point is due the possibility of condensation. Condensation can occur at the leading edge of the inducer. As the flow enters the eye and flows over the leading edge it accelerates. The acceleration is commonly observed on the suction side of the vane. The acceleration in that region can cause the static conditions to fall into the two-phase region which can lead to condensation. 
The effects of two-phase flow and condensation were studied by MIT Gas Turbine Laboratory (Baltadjiev, 2012). As expected, they concluded that in regions where centrifugal compressor experience flow acceleration, the flow can fall into the two-phase region, and lead to condensation. However, they did not find evidence of the two-phase flow affecting the performance of the machine.

Condensation is unlikely to occur because of the difference of two different time scales. The first, is the time required for stable droplets to form and is defined by the rate of nucleation (Baltadjiev, 2012). The second time-scale is the residence time of the flow. It was found that the time required for droplets to form is much larger than the residence time of the fluid in the low-pressure region (Baltadjiev, 2012). Similarly, nucleation in high-speed flows generally happens at subcooled conditions. These subcooled conditions are needed to overcome the energy barrier before spontaneous condensation can lead the fluid back to nearly equilibrium conditions (Baltadjiev, 2012). However, this phenomenon needs further analysis and modelling near the critical point.

Sandia Laboratories were one of the first to stablish an operational pilot $\mathrm{S}-\mathrm{CO}_{2}$ loop and perform extensive tests. In their 2010 report (Wright et. al, 2010), they mentioned that the compressor was tested in the two-phase region. They noticed fluctuations in density and mass flow, but no effects were measured regarding power. This suggests that two-phase flow may not extensively affect the compressor. In another brief note, they concluded that the results of the compressor experiments were in good agreement with their predictions (Wright et. al, 2009).

A parameter that indicates the possibility of condensation occurring was published by the University of Seville, Spain. It is referred to as the Acceleration Margin 
to Condensation (AMC) (Monge et. al, 2014). It is defined as the throat Mach number at which static properties of the fluid lie on the saturation line (Brun et. al, 2017). This is a parameter that may be used by the designer to predict the likelihood of condensation taking place. However, as concluded by Pham et. al, (2016) no quantitative suggestion can generally be given as its relevance would be influence by the geometry and other factors, such as residence time (Baltadjiev, 2012).

\subsection{Initial Sizing}

As mentioned in Chapter 2, the author of this thesis started the study of the centrifugal compressor with a predefined 1D geometry and a fixed rotational speed. The initial design of the impeller is described in Kibsey, 2016. The author's responsibility was to generate a $3 \mathrm{D}$ geometry model, complete the preliminary $3 \mathrm{D}$ aerodynamic study and change the geometry as needed until a satisfactory result was achieved.

The values used in the generation of the first 3D model are summarized in Table 4-2. Figure 4-2illustrates the basic geometry of the centrifugal compressor.

Table 4-2: Initial sizing dimensions. These parameters were used to generate the $1^{\text {st }} 3 \mathrm{D}$ geometry. As the $3 \mathrm{D}$ analysis was completed the geometry was modified several times.

\begin{tabular}{|c|c|c|c|}
\hline \multicolumn{2}{|c|}{ Impeller } & \multicolumn{2}{|c|}{ Vaneless Diffuser } \\
\hline Parameter & Value & Parameter & Value \\
\hline Inlet Hub Radius & $0.25 \mathrm{~mm}$ & Diffuser Length & $97.8 \mathrm{~mm}$ \\
\hline Impeller Tip Radius & $0.542 \mathrm{~mm}$ & $\mathbf{r}_{\text {outlet }} / \mathbf{r}_{\text {inlet }}$ & 2.05 \\
\hline Outlet Radius & $93 \mathrm{~mm}$ & Inlet Width & $10.9 \mathrm{~mm}$ \\
\hline Axial Length & $68 \mathrm{~mm}$ & Outlet Width & $13.1 \mathrm{~mm}$ \\
\hline Outlet width & $10.9 \mathrm{~mm}$ & & \\
\hline Tip clearance & $0.5 \mathrm{~mm}$ & & \\
\hline Backswept Angle & $30^{\circ}$ & & \\
\hline Vane Thickness & Varies* & & \\
\hline
\end{tabular}




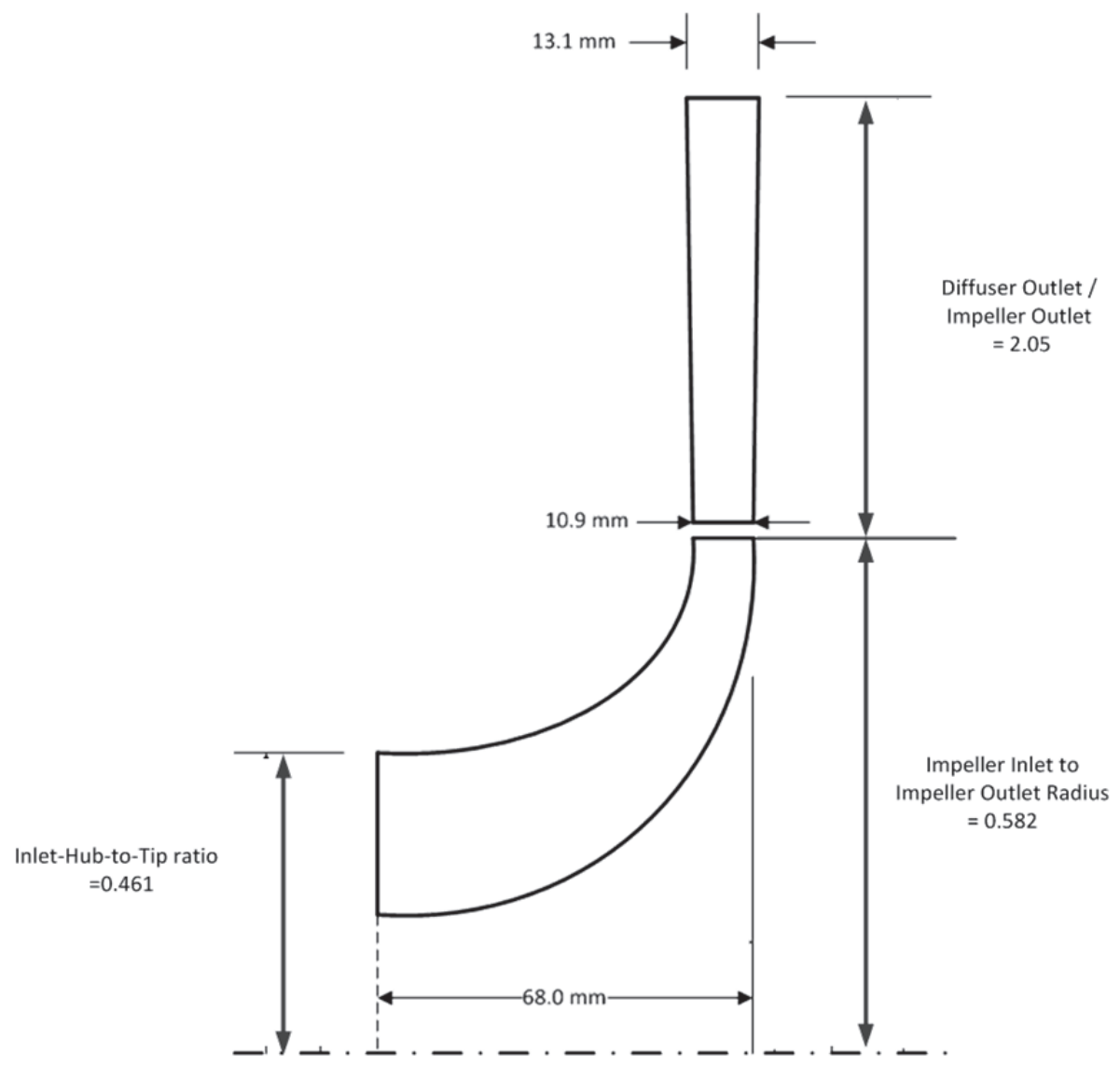

Figure 4-2: Initial dimensions of the centrifugal compressor. Note: curvature of the impeller and diffuser are not to scale.

\subsection{Scaling Methodology and Design Point \#2}

Chapter 3 explained the design process used during the preliminary design of the centrifugal compressor. It was mentioned that dimensional analysis was applied to overcome the convergence challenges in the CFD computation and achieve the 3D aerodynamic analysis. The original plan was to complete the 3D aerodynamic analysis using the conditions of Design Point \#1. However, ANSYS CFX V17.2 experienced convergence issues. Other authors, like Baltadjiev (2012) and Pham et. Al (2016) have also expressed difficulties achieving convergence near the critical region. The 
convergence difficulties are primarily related to the rapidly varying properties and the quality of the RGP file near the critical point. Due to the time constraints of the project, non-dimensional parameters were used to overcome the computational modelling problems. The non-dimensional parameters were employed in the Scaling Methodology. This methodology allows the calculation of the values required to fully define Design Point \#2.

The objective of the Scaling Methodology is to obtain a partially dynamically similar design point for a geometrically similar machine. The geometry is fixed between Design Point \#1 and Design Point \#2, and is therefore geometrically similar. The partially dynamically similar point is referred to as Design Point \#2 and it lies in a region where S$\mathrm{CO}_{2}$ behaves as an ideal gas. Modelling the CFD in this region prevents the computational problems from occurring.

Chapter 3 mentioned that the main difference between Design Point \#1 and Design Point \#2 is the fluid region. Design Point \#1 is in the supercritical regime and behaves as a real fluid, where the compressibility factor $(Z)$ is $\sim 0.24$. On the other hand, Design Point \#2 is still in the supercritical regime but behaves as an ideal gas, where the compressibility factor $(\mathrm{Z})$ is $\sim 0.98$. The temperature and pressure values were determined by using Equation 2.15 and Equation 2.16. The Reduced pressure and Reduced temperature were selected from Figure $2-7$ to achieve a compressibility factor of $Z \approx 1$. This provided a temperature of $600 \mathrm{~K}$ and pressure of $10 \mathrm{MPa}$ for Design Point \#2. The sole purpose of setting the values of Design Point \#2 in the ideal regime was to facilitate the CFD simulation. 
To fully define Design Point \#2, values of mass flow $(\dot{m})$ and rotational speed $(\mathrm{N})$ are needed for the partially dynamically similar design point. The similarity ratios Flow Coefficient $\left(\Pi_{m}\right)$, and Speed Coefficient $\left(\Pi_{N}\right)$ were calculated for Design Point \#1. Then, Equation 2.24 and Equation 2.25 were rearranged to obtain the mass flow and RPM needed at Design Point \#2. Table 4-3 summarize the values for Design Point \#2, while Figure 4-3 illustrates the steps of the Scaling Methodology.

Table 4-3: Summary of Design Point \#2.

\begin{tabular}{|l|l|c|}
\multicolumn{3}{c|}{ Design Point \#2 } \\
\hline \multicolumn{1}{|c|}{ Parameter } & \multicolumn{1}{c|}{ Units } & Value \\
\hline $\mathbf{P}_{\text {0,In }}$ & {$[\mathrm{MPa}]$} & 10.00 \\
\hline $\mathrm{T}_{\mathrm{O}, \text { In }}$ & {$[\mathrm{K}]$} & 600.00 \\
\hline Mass Flow & {$[\mathrm{kg} / \mathrm{s}]$} & 42.903 \\
\hline Rotational Speed & {$[\mathrm{Rev} / \mathrm{min}]$} & 35,100 \\
\hline
\end{tabular}




\section{Scaling Procedure}

Choose Reference point

( $P_{o, \text { Ref }}, T_{o, \text { Ref }}, R P M_{\text {Ref, }}$ Mass Flow Ref $)$

Calculate for reference point

- Speed Coefficient $\Pi_{\mathrm{N}}=\frac{N_{\text {ref }}}{\sqrt{T_{\text {o. Ref }}}} \frac{D}{\sqrt{Z_{\text {ref }} \cdot R_{\mathrm{CO}_{2}}{ }^{*} n_{s_{\text {ref }}}}}$

- Flow Coefficient $\Pi_{\mathrm{m}}=\frac{\dot{m}_{\text {Ref }} \sqrt{T_{\text {o.Ref }}}}{P_{o . R e f}} \sqrt{\frac{2_{r e f} \cdot R_{C O}}{n_{s_{r e f}}}} \frac{1}{D^{2}}$

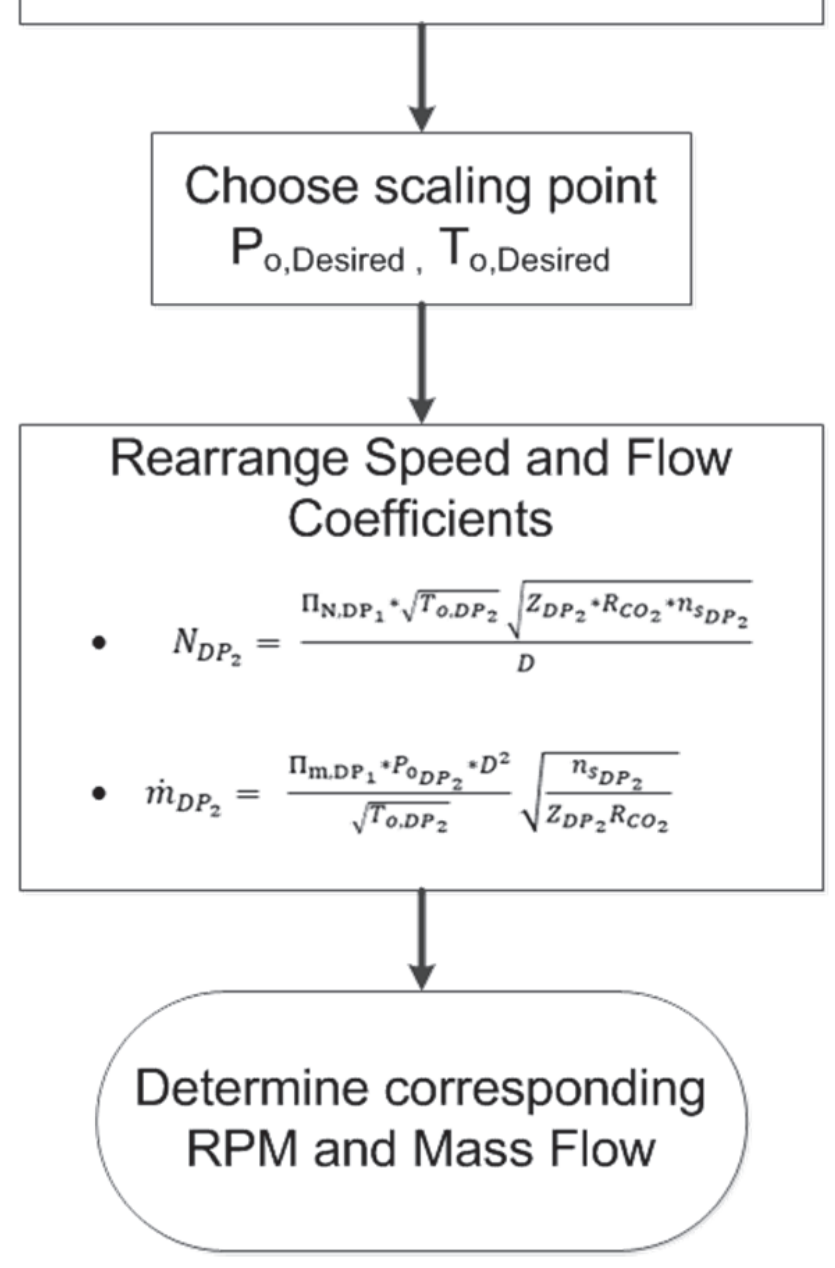

Figure 4-3: Steps required to implement the Scaling Methodology. 
It is important to understand that Design Point \#2 was the operating point used as boundary conditions to perform the $3 \mathrm{D}$ aerodynamic analysis. The results of the aerodynamic analysis then were used to guide any geometry changes. Because Design Point \#2 corresponds to ideal gas behaviour, there is no need to worry about the degree to which the correlations presented in Chapter 2 are valid within the real gas region. Similarly, the CFD analysis encountered less difficulties achieving convergence because the new operating point is partially dynamically similar and it is a geometrically similar. Through similarity, it was expected that the flow would behave in a physically similar manner near the critical point, however as previously discussed the rapid changes in this region may introduce some changes.

It was mentioned in Chapter 2 that the independent non-dimensional coefficient of Reynolds Number was not matched. This parameter is mainly dependent on the rotational speed $(\mathrm{N})$ and diameter $(\mathrm{D})$ of the machine. The rotational speed is fixed through the Speed Coefficient, while the diameter of the machine was a fixed geometry parameter. Therefore, it was not possible to match Reynolds Number. However, as mentioned in Chapter 2, it is well known that the Reynolds Number mainly influences the efficiency of the component as mentioned in Chapter 2, hence the implementation of the correction equation (Equation 2.26). The use of this correction equation is explained in Chapter 7. Table 4-4 summarize the Reynolds Number (Re defined by Equation 2.25) between Design Point \#1 and Design Point \#2. 
Table 4-4: Summary of Reynolds Number between Design Point \#1 and Design Point \#2.

\begin{tabular}{|l|cc|c|} 
& Design Point \#1 & Design Point \#2 & $\mathbf{R e}_{\mathrm{DP} \# 1} / \mathbf{R e}_{\mathrm{DP} \# \mathbf{2}}$ \\
\hline $\mathbf{P}_{0,1}$ & $8.4 \mathrm{MPa}$ & $10 \mathrm{MPa}$ & - \\
\hline $\mathbf{T}_{0,1}$ & 304.15 & $600 \mathrm{k}$ & - \\
\hline $\mathbf{Z}_{0,1}$ & 0.24 & 0.98 & - \\
\hline $\mathbf{R e}_{1}$ & $9.787 \times 10^{9}$ & $3.773 \times 10^{9}$ & 2.59 \\
\hline
\end{tabular}

\subsection{Chapter Summary}

- This chapter presented the operating conditions of Design Point \#1.

- It described the behaviour of density as a function of pressure and temperature, and the impact it can have in the different operating points of the machine.

- The possibility of condensation, and the conditions required for the occurrence of condensation were described.

- The operating conditions of Design Point \#2 were defined and the Scaling Methodology was introduced. 


\section{Chapter 5}

\section{Computational Fluid Dynamics for a Supercritical Carbon Dioxide Centrifugal Compressor}

\subsection{Introduction}

ANSYS Workbench V17.2 was the main tool used for the design of the centrifugal compressor. Five different components from this suite were used to complete the investigation. Section 5.2 describes Turbo Setup ${ }^{\circledR}$. Section 5.3 describes BladeGen ${ }^{\circledR}$, the main CAD tool. Section 5.4 describes TurboGrid ${ }^{\circledR}$, the tool used to generate the mesh. Section 5.5 describes $C F X ®$, which is the main computational fluid dynamic component. Section 5.6 describes CFD Post ${ }^{\circledR}$, the post processing tool. Each section will highlight the benefit of using each independent component in the design process of a centrifugal compressor.

\subsection{Turbo Setup}

Turbo Setup is a tool used to facilitate the creation of the workflows needed for analysing centrifugal compressors. This component holds basic information about the design of the centrifugal compressor, as shown in Table 5-1. Turbo Setup quickly generates the workflow components (Mesh, CFD and Post Processing) and provides basic set up conditions corresponding to a centrifugal machine in each of the components. A typical workflow for a centrifugal compressor is illustrated in Figure 5-1.

One of the main benefits of using this tool is the facility to generate compressor maps. After specifying the design point the user can specify up to 15 different speed lines 
and ANSYS will automatically model each of the specified points along the speed lines. It is important to understand that this tool automatically employs CFX and is not compatible with another CFD packages within ANSYS Workbench, such as FLUENT (ANSYS- TurboSystem User Guide, 2016). Table 5-1 summarize the information specified in Turbo Setup.

Table 5-1: Summary of the information required by Turbo Setup.

\begin{tabular}{|l|l}
\hline Units & SI \\
\hline Target Mesh Node Count & 300,000 \\
\hline Component Configuration & Main Blade only \\
\hline Impeller Tip Configuration & Unshrouded Impeller \\
\hline Total Inlet Pressure & $10,000,000 \mathrm{~Pa}$ \\
\hline Total Inlet Temperature & $600 \mathrm{~K}$ \\
\hline Meanline Angle & $0^{\circ}$ (Default - no swirl) \\
\hline Radial Distribution & Constant Angle \\
\hline Gas Property Model & Real Gas \\
\hline Material Option & Select material \\
\hline Material Name & Carbon Dioxide
\end{tabular}

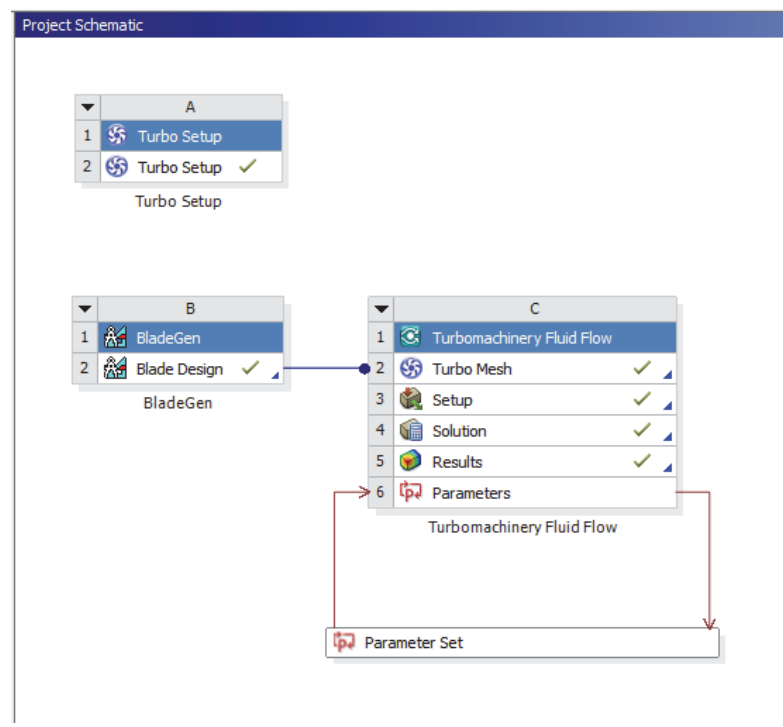

Figure 5-1: Typical workflow of a centrifugal diffuser using Turbo Setup in ANSYS Workbench V17.2. 


\subsection{D Geometry Generation}

\subsubsection{BladeGen}

The 3D geometry of the impeller and diffuser was created using ANSYS BladeGen ${ }^{\circledR}$. This is a tool that allows the designer to create and modify radial or axial turbomachinery.

Recall from Sections 4.3 that the 1D analysis provided the initial size of the machine. The physical sizing of the 3D geometry is based on Design Point \#1. BladeGen requires very few geometry values to generate the first geometry. Table 5-2 summarize the parameters specified to generate the first geometry.

Table 5-2: Summary of the initial geometry sizing parameters, the values presented below were used as inputs in BladeGen to generate the $1^{\text {st }}$ geometry.

\begin{tabular}{|c|c|c|}
\hline Geometry & Impeller & Diffuser \\
\hline Parameter & Value & Value \\
\hline Inlet hub radius $\left(r_{h, i n}\right)$ & $25 \mathrm{~mm}$ & $93 \mathrm{~mm}$ \\
\hline Inlet Shroud Radius $\left(r_{\mathrm{s}, \mathrm{in}}\right)$ & $54.2 \mathrm{~mm}$ & $93 \mathrm{~mm}$ \\
\hline Inlet hub z-axis location $\left(Z_{h, \text { in }}\right)$ & $-68 \mathrm{~mm}$ & $0 \mathrm{~mm}$ \\
\hline Inlet shroud $\mathrm{z}$-axis location $\left(\mathrm{Z}_{\mathrm{s}, \mathrm{in}}\right)$ & $-68 \mathrm{~mm}$ & $-10.1 \mathrm{~mm}$ \\
\hline Outlet hub radius $\left(r_{h, o u t}\right)$ & $93 \mathrm{~mm}$ & $190.8 \mathrm{~mm}$ \\
\hline Inlet Shroud Radius ( $\left.r_{\mathrm{s}, \text { out }}\right)$ & $93 \mathrm{~mm}$ & $190.8 \mathrm{~mm}$ \\
\hline Inlet hub z-axis location $\left(\mathrm{Z}_{\mathrm{h}, \mathrm{out}}\right)$ & $0 \mathrm{~mm}$ & $0 \mathrm{~mm}$ \\
\hline Inlet shroud $\mathrm{z}$-axis location $\left(\mathrm{Z}_{\mathrm{s}, \text { out }}\right)$ & $-10.1 \mathrm{~mm}$ & $-10.1 \mathrm{~mm}$ \\
\hline Wrap angle & $45^{\circ}$ & \\
\hline Number of blades & 16 & \\
\hline Number of Splitters & 0 & \\
\hline Hub - Blade thickness (LE-TE) & $2.5-1.68 \mathrm{~mm}$ & \\
\hline Shroud - Blade thickness (LE-TE) & $1.5-1.75 \mathrm{~mm}$ & \\
\hline
\end{tabular}

Figure 5-2 illustrates the graphical-interface of BladeGen which is composed of 4 different windows. The 2D meridional geometry (top left), the blade contour (top right), the blade angle distribution (bottom left) and blade thickness (bottom right). 
By default, BladeGen generates 3 layers (hub, mean line and shroud). Each layer corresponds to a location along the span-wise of the blade; more layers can be added if desired. The designer can toggle between each layer and specify specific parameters that define the blade at that span-wise location.

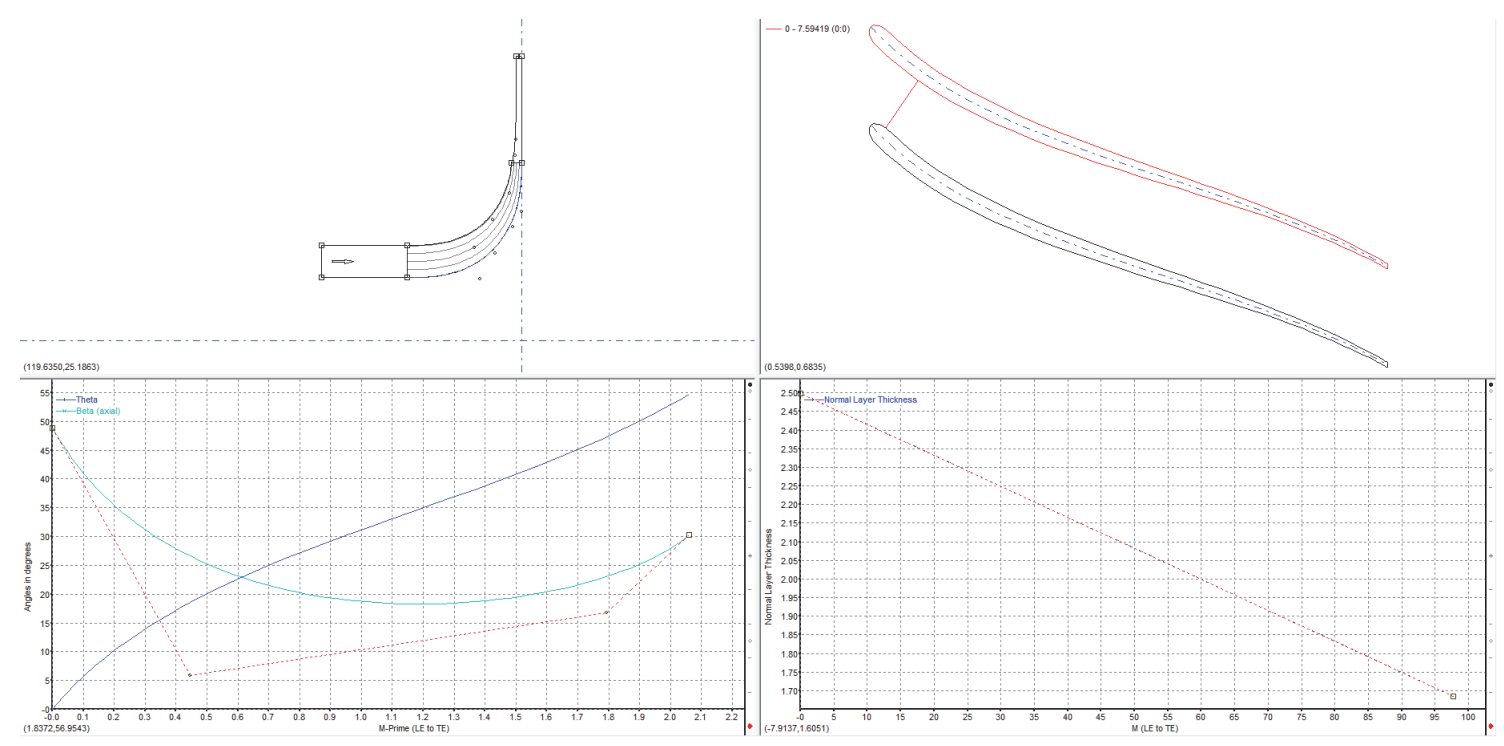

Figure 5-2: BladeGen graphical interface. (Top Left) illustrates the meridional view of the compressor and highlights the actual geometry of the inlet, impeller and diffuser. (Top Right) illustrates the actual geometry of the blades. (Bottom Left) provides the angles used to design the blades. (Bottom Right) allows the user to define the blade thickness.

The tip clearance is specified as a layer and the corresponding tip clearance is reduced from the blade height rather than the inlet shroud radius. The tip clearance specified in this design corresponds to $0.254 \mathrm{~mm}$. It is important to understand that the tip clearance can be defined in BladeGen (ANSYS - TurboGrid User Guide Blade Editor, 2016) as despite of some authors having stated that it is not possible. 
The 2D meridional geometry (Top Left) is where the user modifies the geometry. The geometry is mainly defined by specifying points relative to the radius (r) and z-axis (Z). However, more complex methods can be employed to create the desired curvature.

The angles of the blade (Bottom Left), in the context of this project, are defined by a "Beta Definition". This definition provides the flexibility of defining the LE and TE Beta angles. This method defines the Theta (Blue) by the Beta (Cyan) curve with the initial theta being fixed to $0^{\circ}$ at the LE (ANSYS - BladeGen Help Menu, 2016). Beta ( $\beta$ ) corresponds to the slope of the blade and Theta $(\theta)$ to the angular placement of the blade. The number of points defining the Beta curve is specified by the user and depends on the selected curve defining method. The different methods available to define the curve are (1) Cubic spline segment (2) Best fit polynomial segment (3) Piecewise linear segment and (4) Bezier segment. For the design purpose of this project the Bezier segment was employed.

The thickness profile can be defined using the same methods available for the blade curve definition previously presented. As shown in Table 5-2, the blade thickness was varied linearly between the LE and TE as well as the hub and shroud.

\subsection{Mesh Generation for Centrifugal Turbomachinery}

\subsubsection{TurboGrid}

ANSYS Workbench includes TurboGrid, a mesh-generation tool specifically designed for turbomachinery. This tool is automatically used when Turbo Setup is employed. TurboGrid is a meshing tool that generates hexahedral (H-Grid) meshes (ANSYS - Intro Turbo Grid, 2016), ANSYS - TurboGrid User Guide, 2016). Hexahedral 
meshes are commonly described as a topological cubes which have 8 vertices and 12 edges bounded by 6 quadrilateral faces. It is known that the accuracy of solutions using hexahedral meshes is the highest (Benmeddour, 2017).

TurboGrid has a large degree of autonomy, which leads to faster meshing time. When used in conjunction with Turbo Setup, the software automatically detects the type of turbomachinery being imported and generates the mesh in a time efficient manner.

TurboGrid high performance is mainly attributed to its topology. The grid is divided into different structured blocks that act as a framework for positioning the mesh. The blocks are positioned by default to achieve the desired grid conditions. Each topology block handles the details of the topology surrounding a blade (ANSYS - TurboGrid User Guide, 2016). Each block contains a specific number of mesh elements. The mesh elements vary in size across each of the topology blocks to achieve a smooth transition between blocks. The smooth transition is achieved by node biasing, which also helps reduce the number of mesh elements. The most common topology method employed in TurboGrid is ATM Optimized with an automatic method.

Turbo Setup automatically imports the target node mesh count previously specified, however, the user can override this value and specify a coarser or finer mesh value. The meshes used throughout this investigation consisted of $\sim 740,000$ nodes and $\sim 680,000$ elements. Chapter 6 will summarize the grid sensitivity analysis performed. However, it was concluded that a grid with a density of $\sim 740,000$ nodes and $\sim 680,000$ elements provided accurate enough results within an acceptable computation time. 
TurboGrid allows the user to specify parameters at different regions of the mesh such as the Inlet, Outlet, shroud Tip, Hub Tip and passage. Each tab provides the user with control of the grid conditions within each block.

$\mathrm{Y}+$ is another important parameter to consider when creating the mesh. The $\mathrm{Y}+$ value is a non-dimensional parameter defining the distance of the first node from the wall. However, it is important to understand that TurboGrid and CFX have their own built in definitions for $\mathrm{Y}+$. TurboGrid provides a definition specifically targeted to turbomachinery. The formula used in TurboGrid that relates the wall distance to $\mathrm{Y}+$ is summarized by Equation 5.1 (ANSYS - TurboGrid User Guide, 2016).

$$
\Delta y=L \Delta y^{+} \sqrt{80} R e_{x}^{\frac{1}{14}} \frac{1}{R e_{L}}
$$

Where $\mathrm{L}$ is the blade chord, $\Delta y^{+}$is the specified target value $y^{+}, R e_{x}$ is the Reynolds Number based on the distance along the chord (measured from the leading Edge), and $R e_{L}$ is the Reynolds Number based on the chord length (ANSYS - TurboGrid User Guide, 2016). It is important to understand that TurboGrid approximates $\mathrm{L}$ as the algebraic average of the chord length of each blade. The user must specify $R e_{L}$ and TurboGrid approximates $R e_{x}=R e_{L}$ (ANSYS - TurboGrid User Guide, 2016).

This investigation focused on only using the wall functions in CFX. As shown by Pham et. al (2017) and ANSYS - TurboGrid User Guide (2016) the wall functions provide accurate enough results and it is not necessary to apply the $\mathrm{Y}+$ distance. However, when surface roughness is introduced, it is recommended that $\mathrm{Y}+$ be investigated. The results of this investigation, had $\mathrm{Y}+$ values that varied along the span of the blade, hub and shroud. The $\mathrm{Y}+$ values varied between $\mathrm{Y}+=1$ and a maximum of $\mathrm{Y}+$ 
$\sim 120$ in areas of interest. Figure 5-3 to Figure 5-7 illustrate the mesh that was used in the aerodynamic analysis of the final geometry.

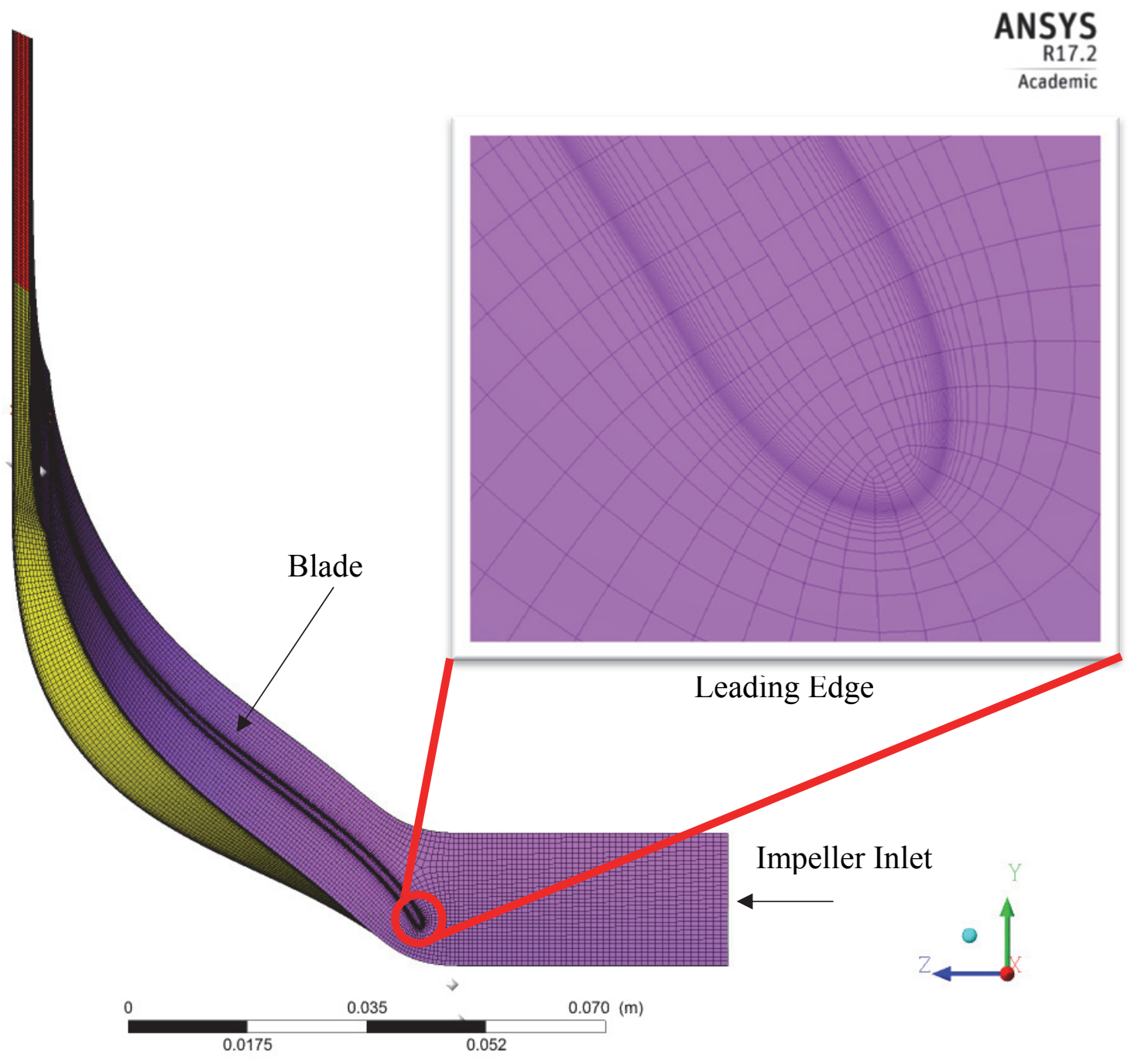

Figure 5-3: Mesh of the impeller and blade at the shroud. 


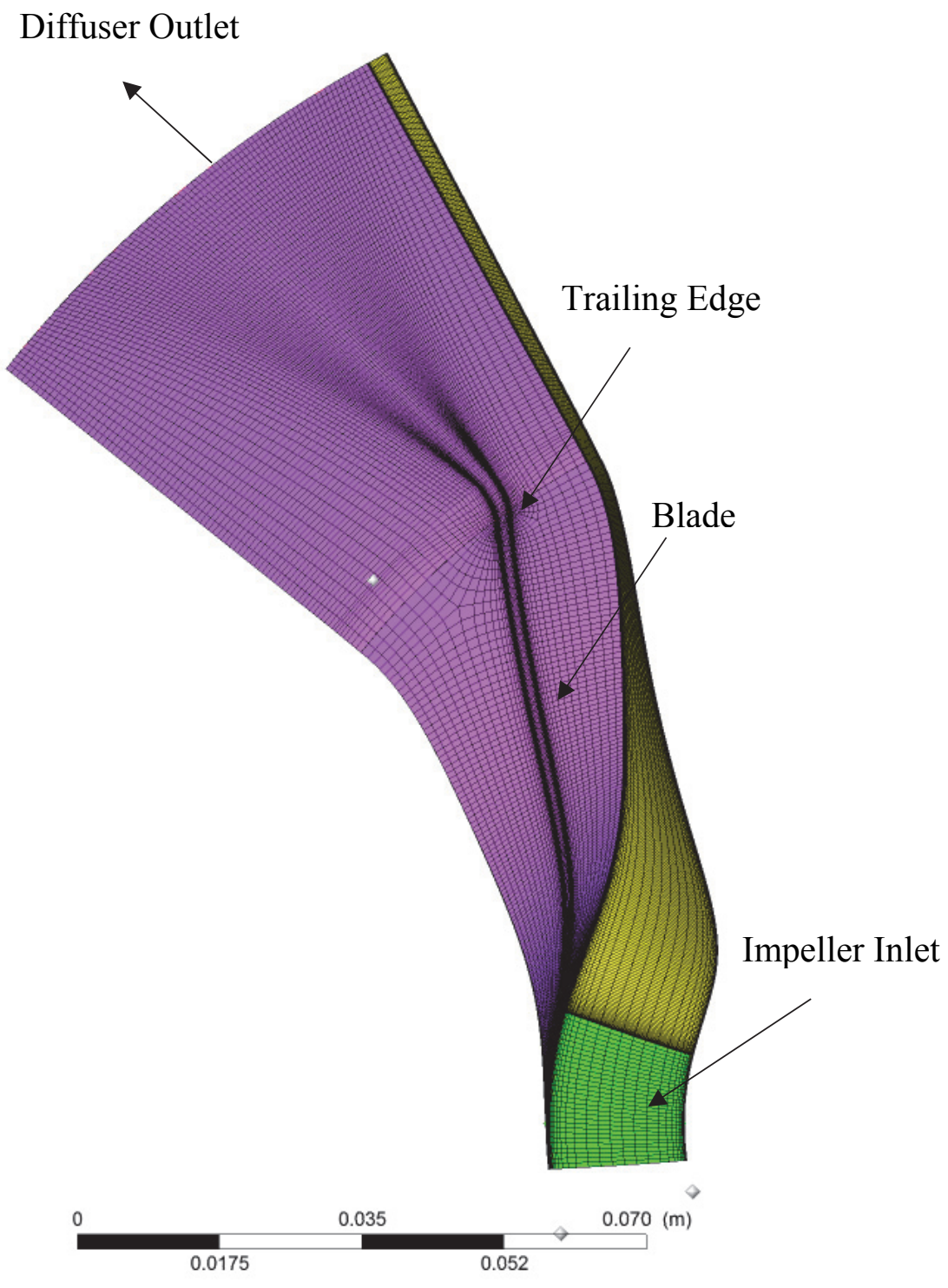

ANSYS

R17.2

Academic

\section{Trailing Edge}

Figure 5-4: Mesh of the impeller, blade and diffuser at the shroud. 


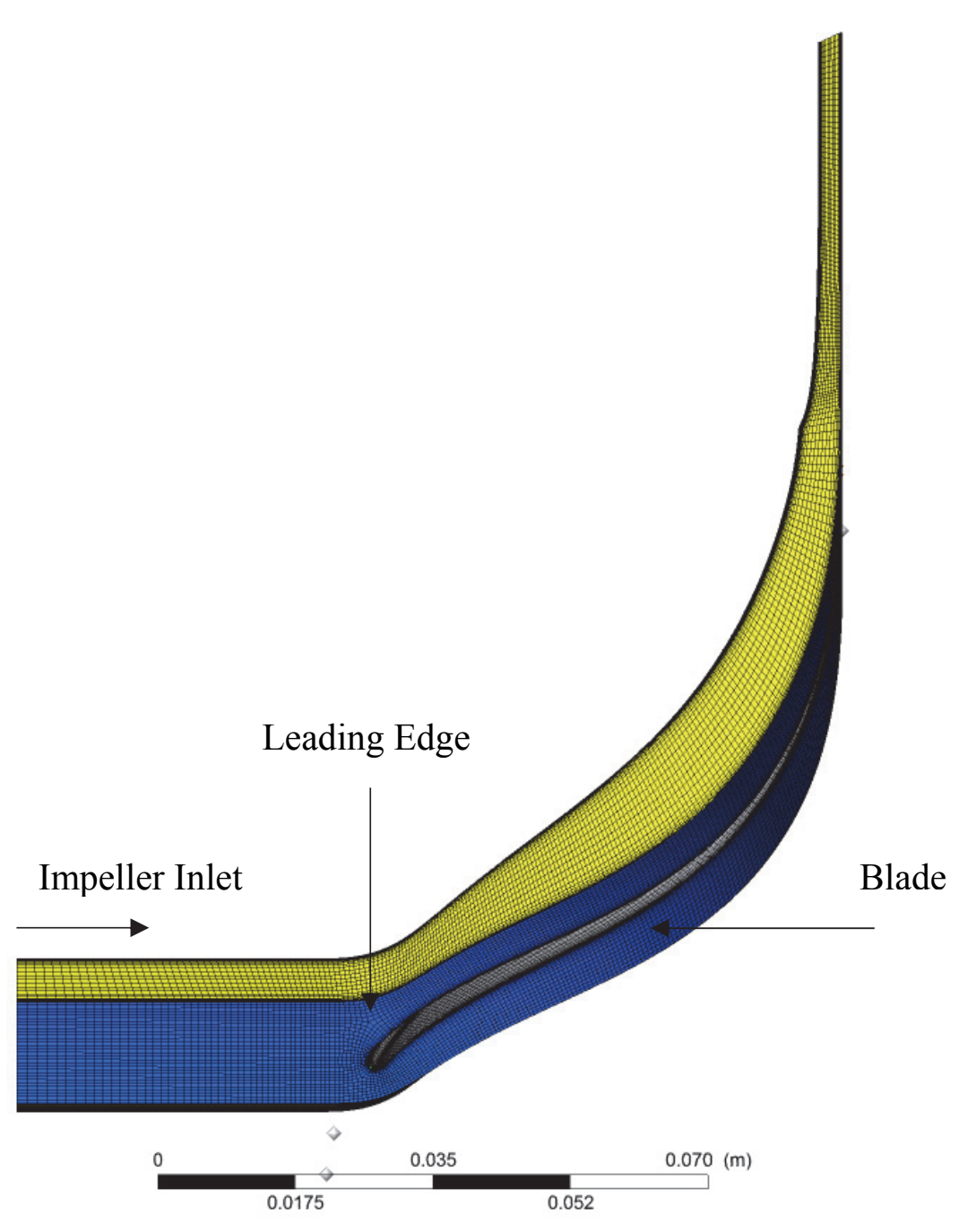

ANSYS

R17.2

Academic

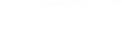

Figure 5-5: Mesh of the inlet and impeller at the hub. 


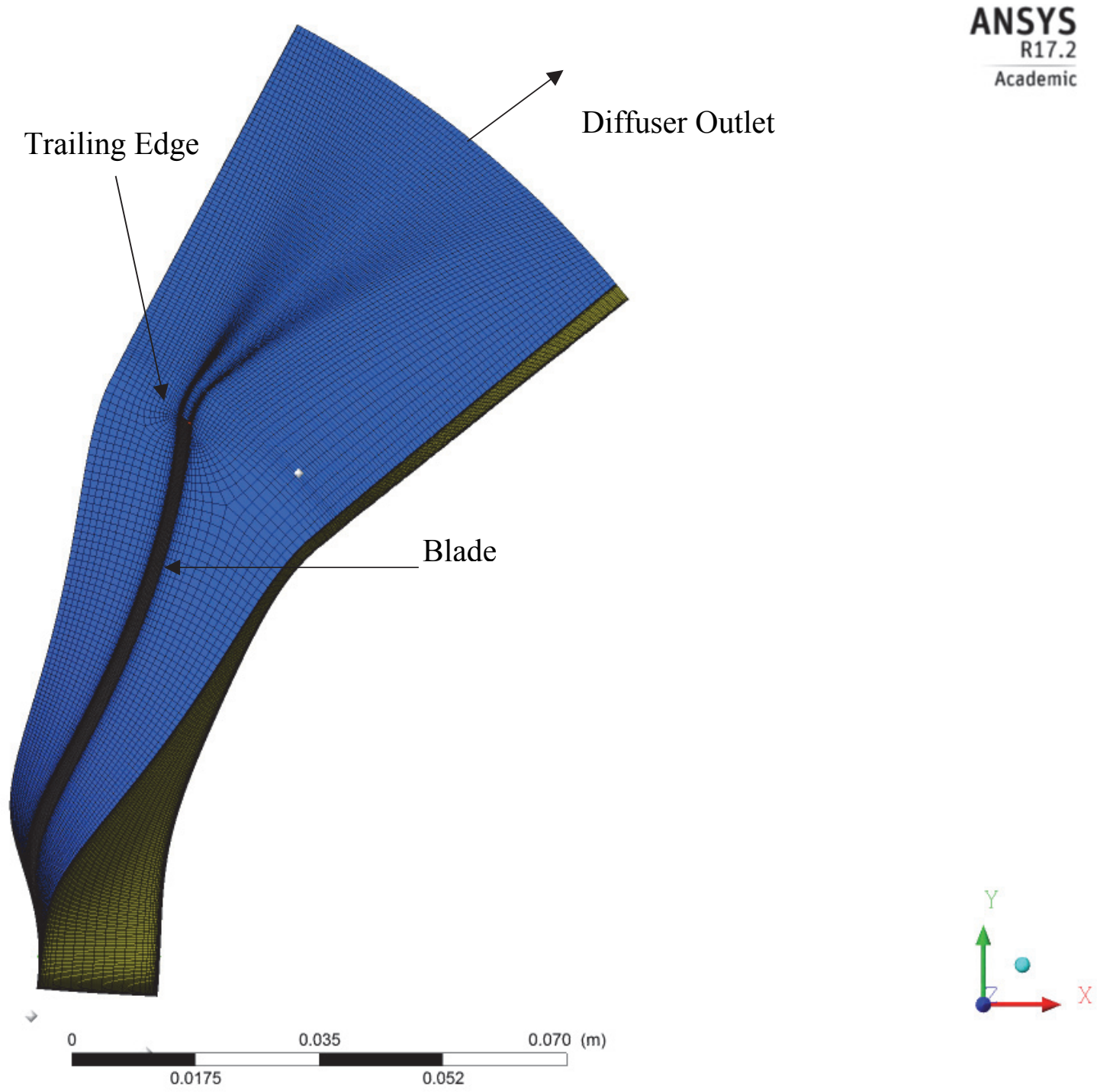

ANSYS

R17.2

Academic

0.0175

Figure 5-6: Mesh of the impeller, blade and diffuser at the hub. 


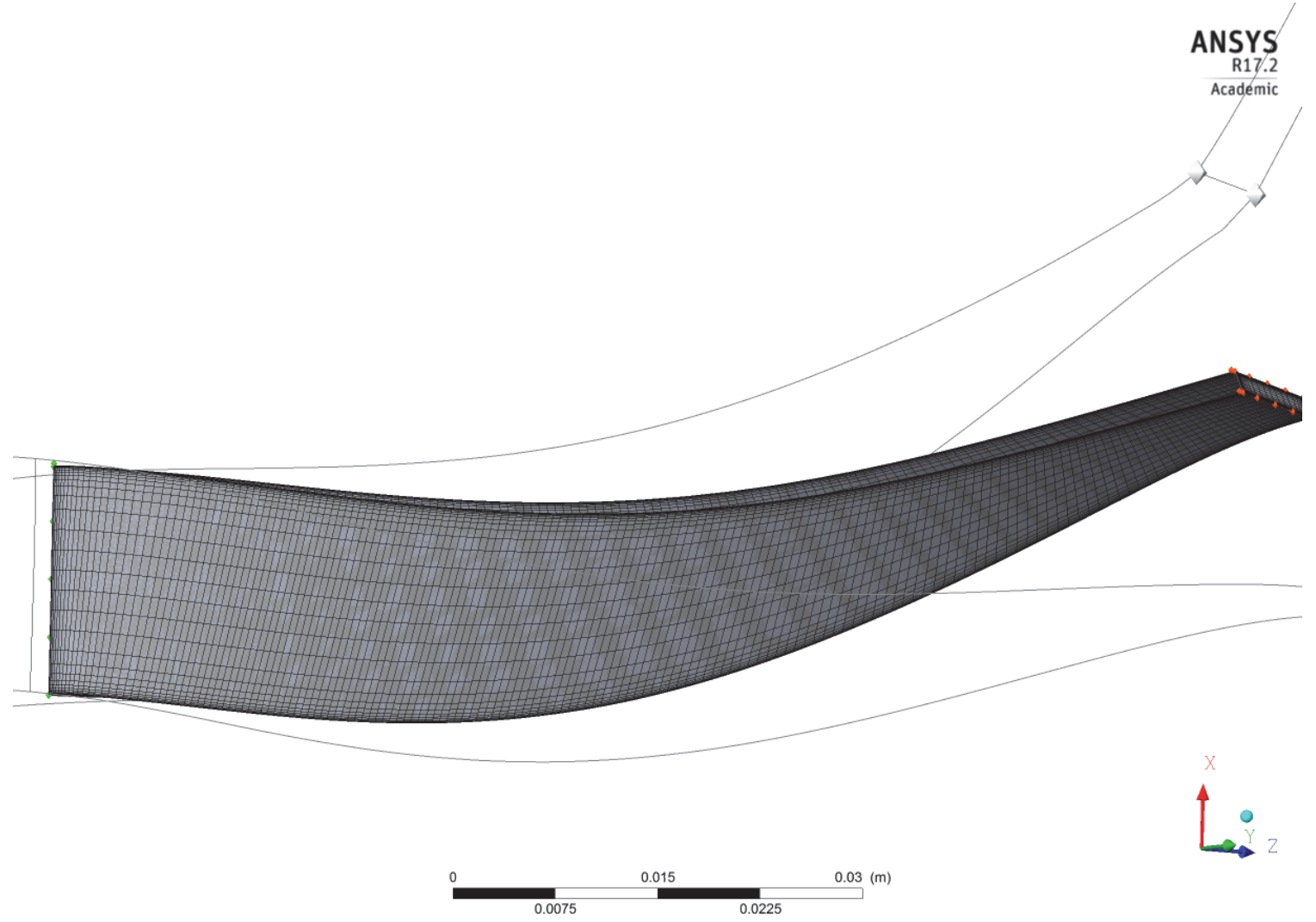

Figure 5-7: Mesh of the blade and impeller passage.

\subsection{D Aerodynamic Analysis}

\subsubsection{ANSYS CFX}

ANSYS CFX is a computational fluid dynamic software. CFX is known to be a preferred tool for modelling turbomachinery. CFX is a 3D code that uses the control volume finite element method. ANSYS CFX solves Reynolds Averaged Navier-Stokes (RANS) equations. In the case when a 2-equation turbulence model is used, then the two equations are solved along the RANS equations. For this investigation, the two-equation $k-\omega$ shear stresss transport (SST) turbulence model was employed. 


\subsubsection{Boundary Conditions}

The simulation is set up by implementing the Turbo mode. This mode is aimed at turbomachinery and it automatically creates the boundary conditions and interfaces of the component. The Turbo mode is made to complement TurboGrid. The user also has the flexibility to manually create the boundary conditions (BC) and interfaces of the system, however this takes more time. The location of the boundary conditions and interfaces is illustrated in Figure 5-8 and Figure 5-9.

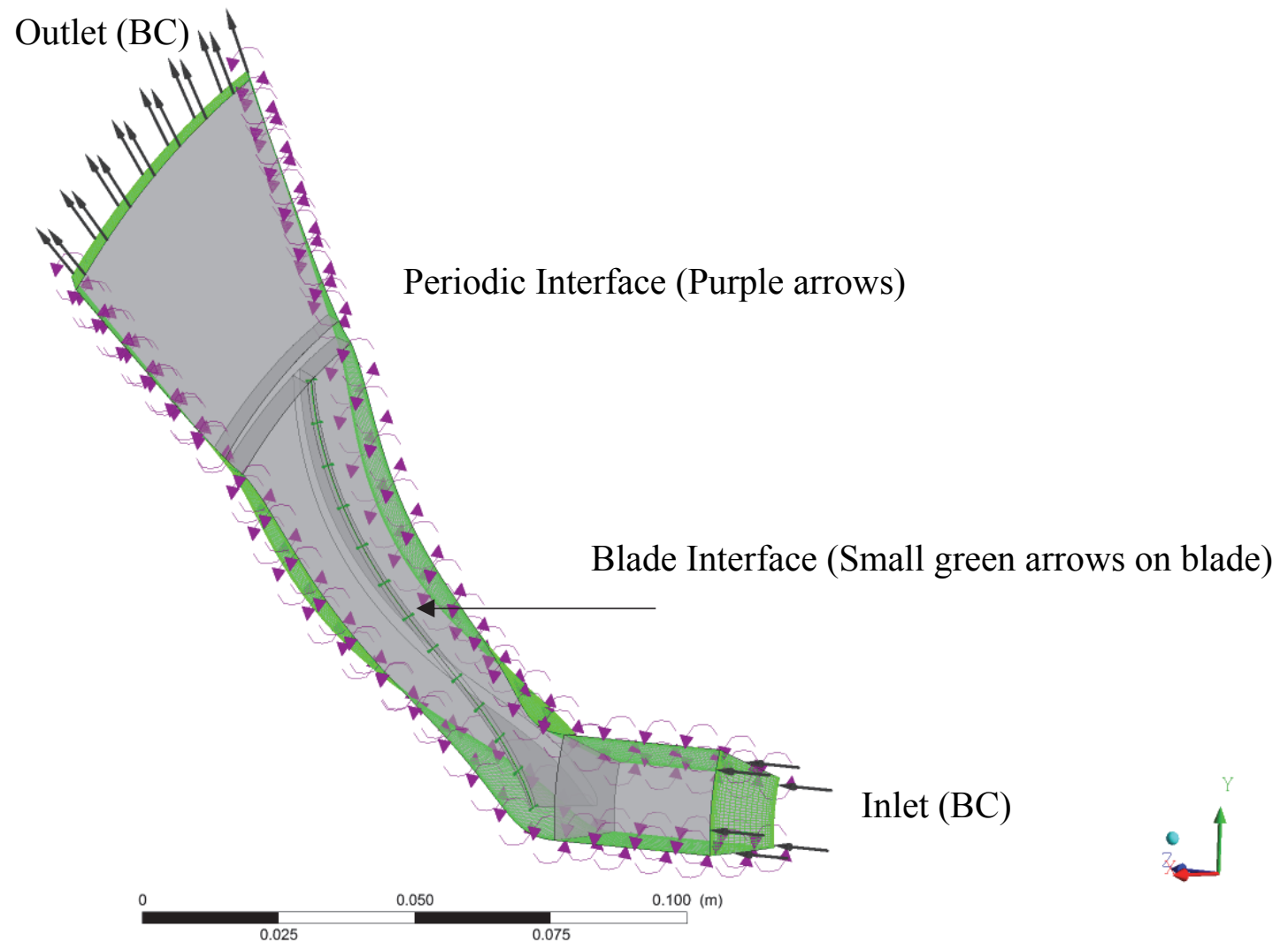

Figure 5-8: Location of the Inlet and outlet boundary condition as well as the location of the different interfaces. 


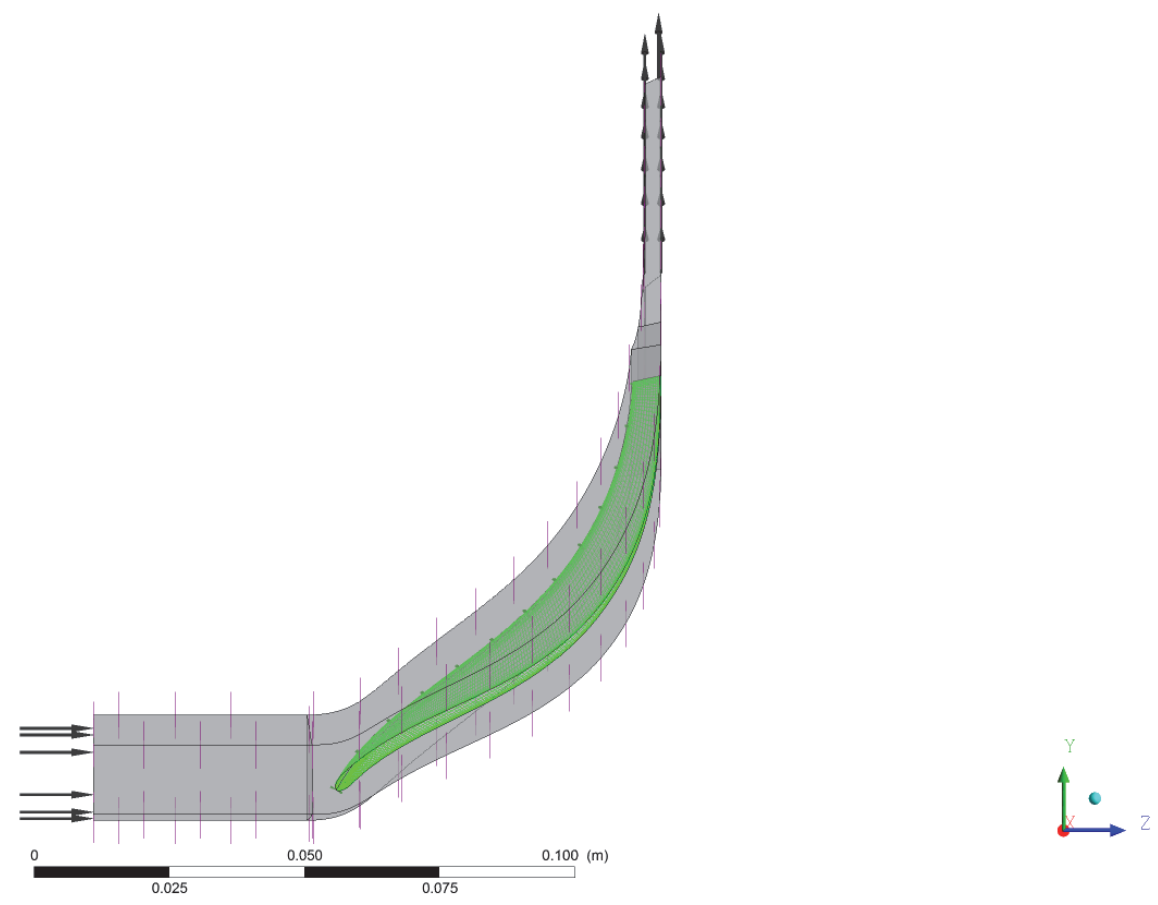

Figure 5-9: Location of the blade boundary.

The basic settings of the system were set to a reference pressure of $0 \mathrm{~Pa}$, and a rotational speed of 35,100 rpm. A total energy heat transfer model was selected as well as the inclusion of viscous work. As mentioned before, the Shear Stress Transport (SST) turbulence model was selected along with the high speed (compressible) wall heat transfer model. The inlet boundary conditions were defined in terms of the total inlet pressure and temperature. The inlet flow was defined to be fully axial (perpendicular to the inlet plane, no swirl). Furthermore, the inlet boundary condition was defined as stationary, and the turbulence intensity was left as the default of $5 \%$. The outlet boundary condition was defined in terms of the outlet mass flow with an active mass flow outlet constraint and the boundary is set to stationary. The entire investigation assumed a smooth wall for the wall roughness. The boundary conditions corresponding to the moving part of the impeller were set as rotating, while the parts such as diffuser, extended inlet, and shroud were specified as a counter rotating wall. Interfaces were set 
up within the tip clearance region. Symmetry was be employed to simplify the modelling of the component, providing the benefit of having to model just one passage. In a similar manner, periodicity allowed the modelling of turbomachinery to be simplified, and rotational periodic interfaces were set up on the outer sides of the passage.

It is common that the convergence is guided by the root mean square (RMS) with values of $1 \times 10^{-5}$ (Baltadjiev, 2014). This investigation used RMS values of $1 \times 10^{-6}$ for the Momentum, Mass and Energy residuals.

CFX has the option of defining an interrupt control, which is used to automatically stop a solver run. This tool is highly beneficial when determining the performance map of the machine. The interrupt control allows ANSYS to run through all the specified points. The specified points corresponded to the different operating conditions in terms of mass flow and rotational speed and they were specified through Turbo Setup.

The interrupt control is independent of the convergence criteria previously specified. The interrupt controls are specified using logical expressions. Pressure ratio and efficiency were the two parameters used to define the interrupt criteria. The standard deviation corresponding to each parameter was calculated. The data used to calculate the standard deviation corresponded to the number of iterations in the run. It was defined that the standard deviation for efficiency had to be less than 0.005 and the standard deviation for pressure ratio had to be less than 0.00025 . The values of standard deviation are specified within the Interrupt Control Function. Similarly, in the logical expression, one defines the number of Current Time Step, and Accumulated Time Step. For this investigation, the Current Time Step was specified as $>10$ and the Accumulated Time 
>30. When all four parameters were true (Standard deviation of PR and Efficiency, as well as the Current Time Step, and Accumulated Time Step) then the interrupt criterion is automatically activated and ANSYS CFX moves on to the next operating point. With these control conditions, it was found that operating points in a well-behaved area achieved convergence (proper residuals). The points near surge or choke region indicated incomplete Interrupt Controls and uncovered solutions; more details are presented in the next chapter.

\subsection{Post-processing}

\subsubsection{CFD-Post}

CFD-Post ${ }^{\circledR}$ is the common post-processor for all ANSYS fluid dynamic packages. This package is a graphical and quantitative post processing tool. Similar to the previous software packages employed in this investigation CFD-Post has a setting aimed at turbomachinery. Turbo Workspace is a setting used to improve and speed up the post processing for turbomachinery (ANSYS - CFD Post User Guide, 2016). Turbo Workspace has an option to generate reports that summarize the performance of the component. These reports are derived from built in templates and are aimed to provide a quick summary of several parameters. Each of the reports are aimed at a different type of turbomachinery such as pumps, axial machinery or radial machines with compressible flow. For this project, Centrifugal Compressor Blade Row Report Beta was used. Similarly, other parameters and areas were investigated as it will be shown in Chapter 6 . 


\section{Chapter 6}

\section{Analysis and Discussion of the 3D Aerodynamic Result}

\subsection{Introduction}

Chapter 6 presents the 3D aerodynamic results corresponding to Design Point \#2. Section 6.2 presents the results of an extended convergence criteria analysis which focus on identifying a trend between the convergence of the solution and the quality of the results. Section 6.3 presents a summary of the initial design geometry. Section 6.4 presents a summary of the grid sensitivity analysis. Section 6.5 presents the findings of the aerodynamic study as well as the results of the final geometry. Section 6.6 presents the compressor map corresponding to Design Point \#2. Chapter 7 will then present the complete compressor performance map for Design Point \#1.

\subsection{Convergence Criteria}

Chapter 5 introduced the convergence criteria that a solution needed to satisfy before it was completed. Recall that for this investigation, solutions needed to achieve an RMS value of $1 \times 10^{-5}$ or less, however, most commonly solutions achieved a value of $1 \times 10^{-6}$. A quantitative check was further performed to try to find a relation between the convergence of the solution (RMS) and the quality of the results. The analysis focused on measuring the percent error between the user specified mass flow rate (outlet boundary) and the mass flow rate obtained by the solution at the inlet boundary.

Recall that the analysis used Turbo Setup ${ }^{\circledR}$. This approach automatically analyzes all of the user specified points along each speed line. At times, the solution did not 
achieve the desired convergence of $1 \times 10^{-6}$, instead, it achieved a residual of $1 \times 10^{-4}$. When this happened, the solver reached the specified maximum number of iterations. It was also observed that these results showcased a divergent trend. Even when convergence was not achieved, Solver still output the results of that specific solution. If Turbo Setup is used to visualize the compressor map it automatically indicates which points did not reach the specified convergence. However, because the values were analyzed in Excel, this analysis was used to further filter the outputs from ANSYS CFX, and ensure only the converged values were used in the data post processing. Instead of performing the percent error analysis, another option is to manually check the Solver convergence or the CFX output file for each solution. However, when creating compressor maps this may involve several hundred operating points. Similarly, when designing a geometry, it may not necessarily be clear when the stall and choke regions are achieved by each speed line. As presented next, this analysis found a trend between the mass flow percent error and the quality of the solution, which provides further information about the limits of the speed line.

The analysis used the CFX output variable $m f r$. This output provides the mass flow at the inlet of the impeller (it can also be used by the user to find the mass flow at any other location). The percent error, defined in Equation 6.1, was calculated relative to the user specified mass flow at the outlet ( $\left.\dot{m}_{\text {reference }}\right)$ and the CFD output value of $m f r\left(\dot{m}_{m f r}\right)$. Recall that the mass flow at the outlet is used as the defining parameter for the outlet boundary condition, therefore, it was always satisfied, even when a poor convergence was achieved.

$$
\epsilon_{m}=\frac{\dot{m}_{\text {reference }}-\dot{m}_{m f r}}{\dot{m}_{\text {reference }}}
$$


The analysis found that when the percent error increased over $\epsilon_{m}>0.1 \%$ the solution tended to have less than ideal convergence, RMS $\geq 1 \times 10^{-4}$. When checking the CFX-Solver Run, it was found that the solutions experienced periodicity (fluctuations) or divergence. In either case, when $\epsilon_{m}>0.1 \%$ the solutions were discarded.

A trend was observed when $\epsilon_{m} \geq 0.1 \%$ the point being modelled tended to be approaching the end of the speed lines, either the "stall" or the "choke" region. This trend can be observed in Figure 6-1, which illustrates the constant speed line corresponding to the design point rotational speed.

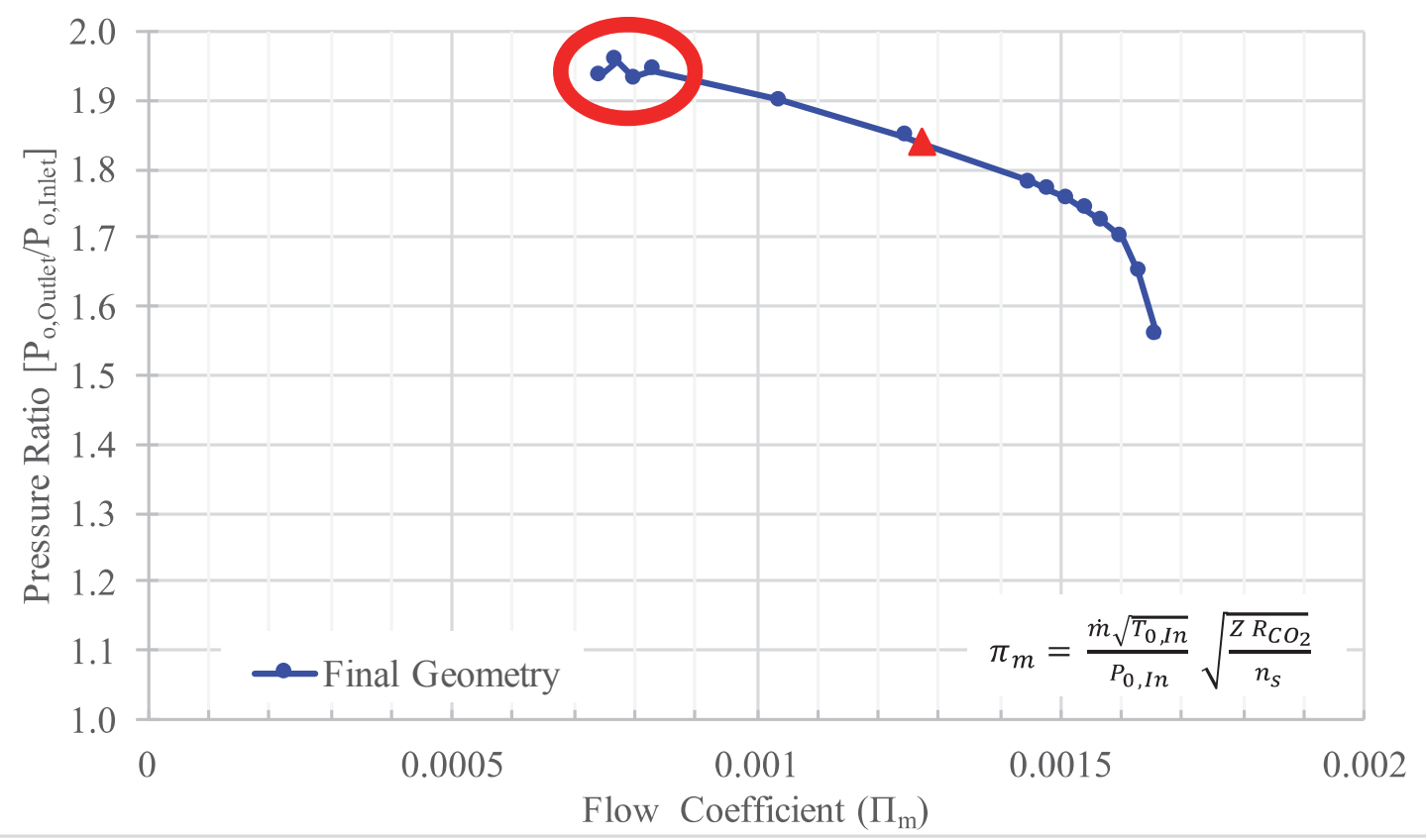

Figure 6-1: Constant speed line performance curve. The speed line corresponds to that of the design point rotational speed. Pressure ratio corresponds to total-to-total pressures. The Flow Coefficient $\left(\Pi_{e}\right)$ is defined by Equation 2.32 .

The $y$-axis corresponds to the total pressure ratio (PR) while the $\mathrm{x}$-axis corresponds to the Flow Coefficient $\left(\Pi_{\mathrm{e}}\right)$. The points near the "stall" region (red circle) 
illustrate a variation in pressure ratio. When the percent error was investigated, as shown in Figure 6-2, it was observed that the $\epsilon_{m}>0.1 \%$ for those same points. A similar trend was observed in the percent error in the "choke" region (orange circles), on the opposite side of the speed line. However, when one looks at the PR it is not possible to directly identify the variation. Therefore, this highlights the importance of further identifying the quality of the solution. Even though the solutions match the trend of the previous points, the solution did not satisfy the convergence criteria.

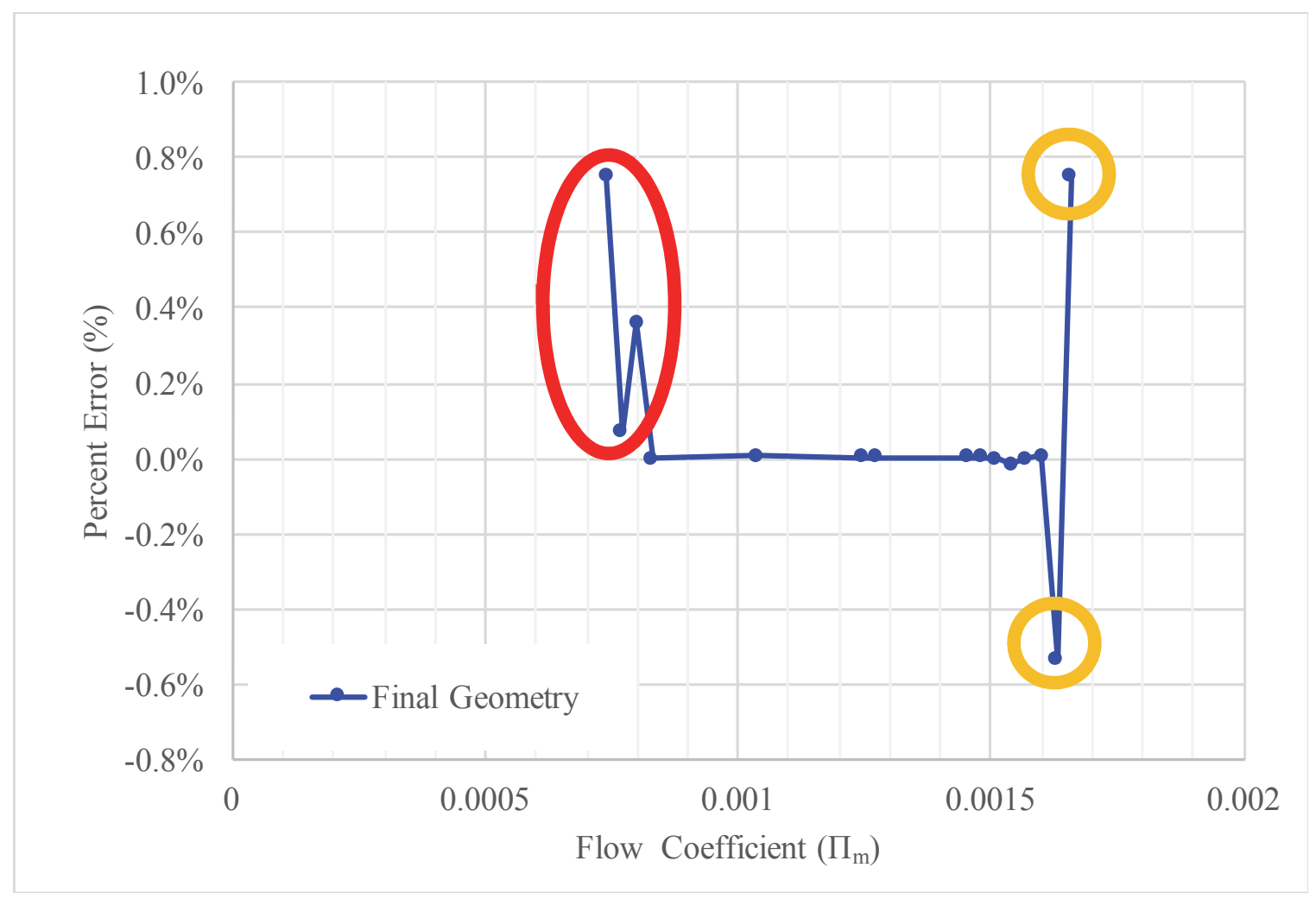

Figure 6-2: Illustrates the percent error $\left(\epsilon_{m}\right)$ of the mass flow. The illustrated percent errors correspond to the design points of the constant speed line illustrated in Figure 6-1. 


\subsection{Initial Design}

At the beginning of the design process the investigation encountered issues with convergence. Later, it was identified that the convergence challenges were attributed to the real gas properties (RGP) and the use of RGP files. The next step was to determine the design quality of the initial geometry (V2). Figure 6-3 illustrates the constant speed line at the design point rotational speed corresponding to the initial geometry (V2).

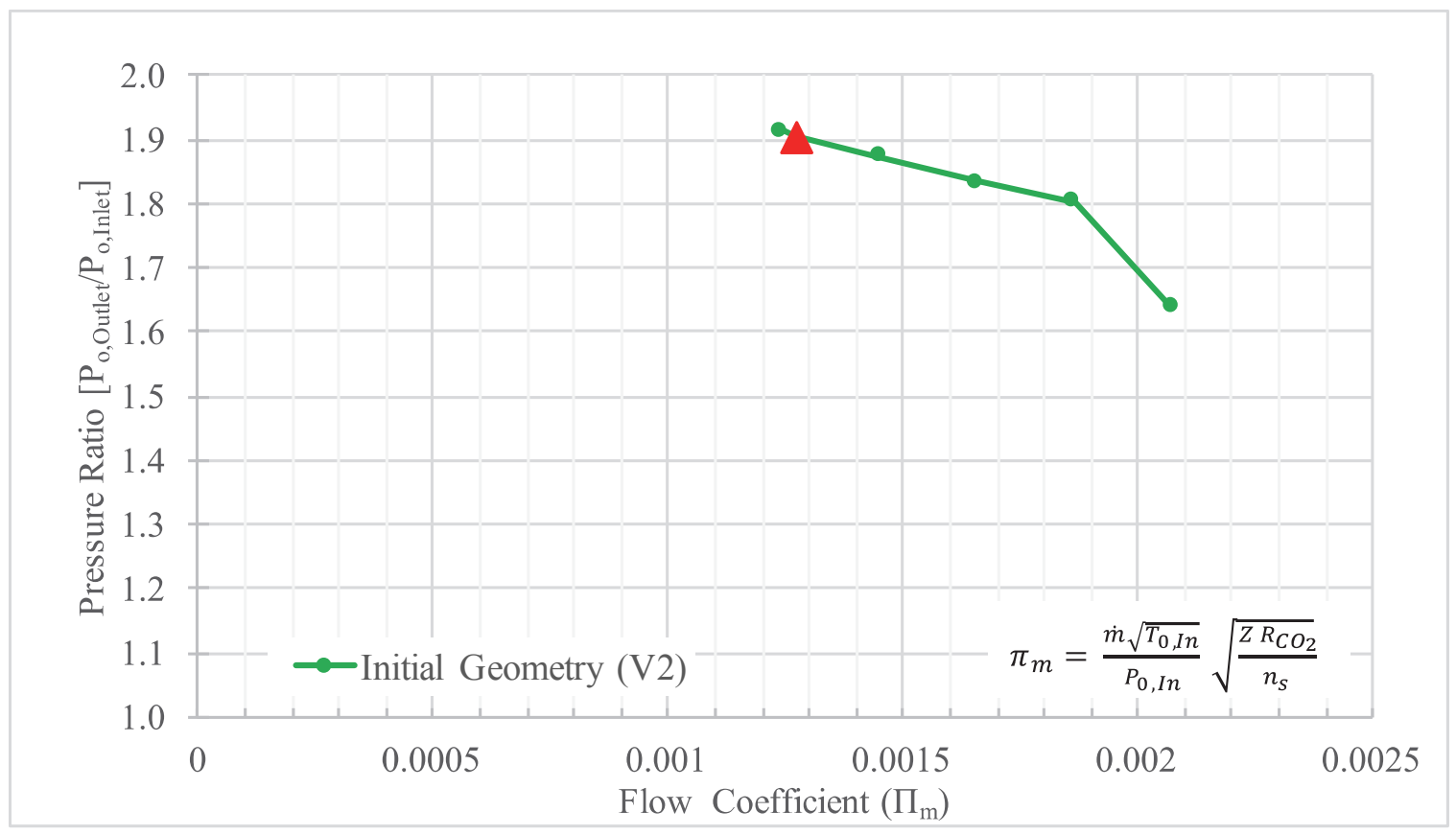

Figure 6-3: Constant speed line performance curve corresponding to the initial geometry (V2). The speed line corresponds to that of the design point. Pressure ratio corresponds to total-to-total pressures. The Flow Coefficient $\left(\Pi_{\mathrm{e}}\right)$ is defined by Equation 2.32.

It was found that the initial geometry (V2) placed the operating point (red triangle) very close to the stall region. As explained in Section 6.2, operating points near the stall and choke experienced poor convergence. Therefore, this set of information provided important conclusions regarding the Flow Coefficient $\left(\varphi_{e}\right)$ presented in Section 
2.9.2. Recall that the Eckardt data suggested that well-designed compressors approach surge at about $\varphi_{e} \approx 0.2$ to 0.25 . For the design point of the initial geometry (V2) it was found that the $\varphi_{e}=0.19$, suggesting that the design was at or close to stall.

Figure 6-4 illustrates the percent error for the same set of operating points. It is possible to observe that despite being close to the surge region the solution did not experience a large percent error $\left(\epsilon_{m}\right)$. Similarly, accepted values of RMS were obtained. However, converged solutions were not possible for lower values of Flow Coefficients. The next steps of the investigation focused on analyzing the grid independence of the results.

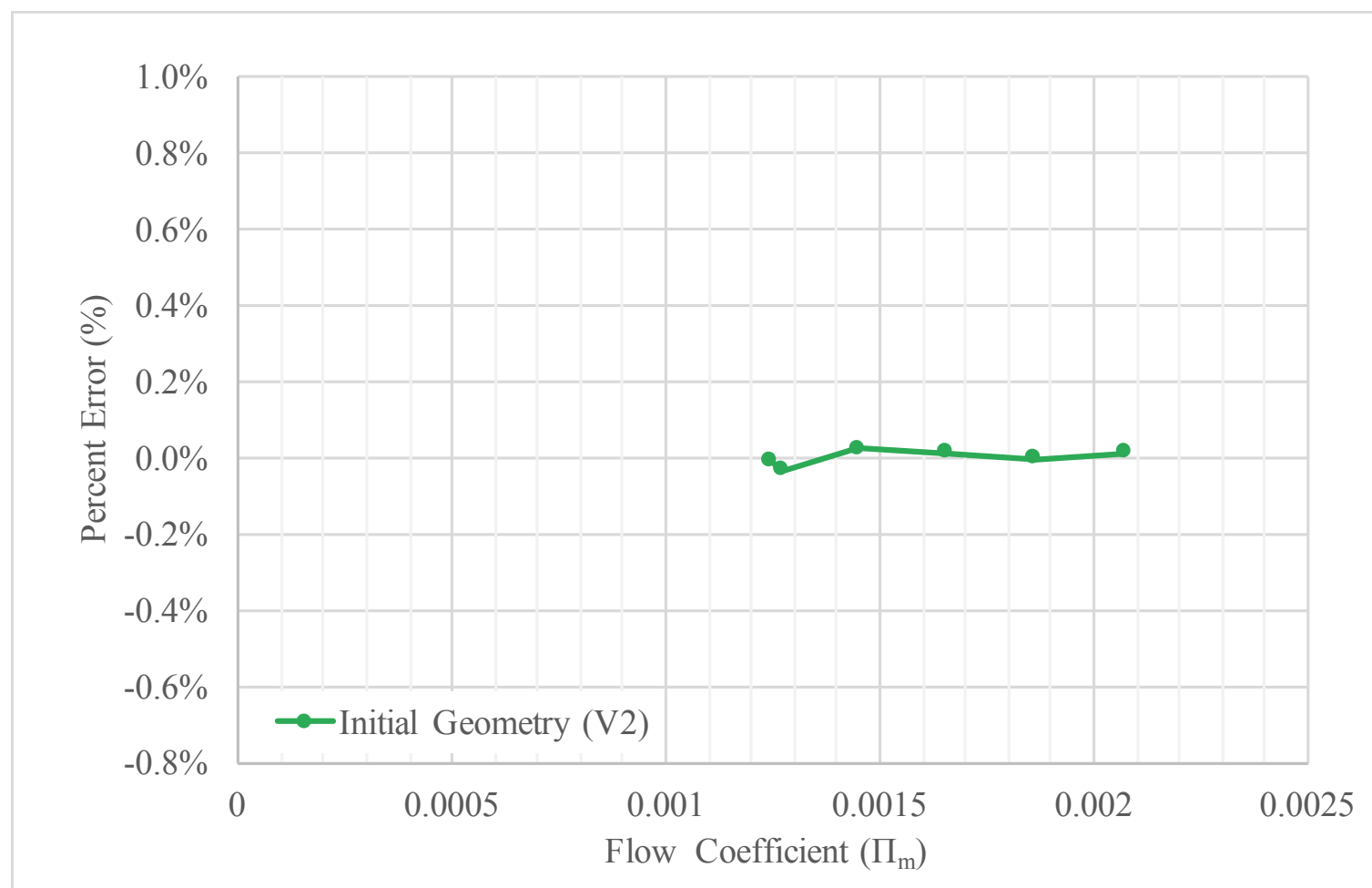

Figure 6-4: Illustrates the percent error $\left(\epsilon_{m}\right)$ of the mass flow. The illustrated percent errors correspond to the design points of the constant speed line illustrated in Figure 6-3. 


\subsection{Grid Sensitivity}

Grid sensitivity analyses are necessary to ensure the results are independent of the grid. Up to date, not many investigations in $\mathrm{S}-\mathrm{CO}_{2}$ have used TurboGrid $\AA$. One of the authors who has employed the same meshing tool (TurboGrid ${ }^{\circledR}$ ) is Monje (2014). Monje investigated several grids ranging from $\sim 26,000$ elements to $\sim 700,000$ elements. Their study concluded that a grid of about $\sim 130,000$ elements provided sufficient results.

The present investigation evaluated several grid densities ranging from $\sim 200,000$ nodes to 1.2 million nodes ( 190,000 elements to 1.1 million elements). It was found that a low-density mesh, despite reaching the RMS, did not experience smooth RMS curves compared to a higher density mesh. It was concluded that a grid with a density of $\sim 740,000$ nodes and $\sim 680,000$ elements provided accurate enough results within an acceptable computation time.

Figure 6-5 summarizes the mass flow percent error $\left(\epsilon_{m}\right)$. Despite the sharp changes in percent error between each grid density, the magnitude of the percent error is observed to be small. The mass flow percent error $\left(\epsilon_{m}\right)$ never exceeded an absolute value greater than $0.01 \%$.

Figure 6-6 illustrates two important performance parameters, pressure ratio and efficiency. These values were not only important for the design of the component itself but they were the main parameters used by the interrupt controls in CFX-Solver as explained in Section 5.1.1. For this reason, it was important to determine their grid independence. It is possible to see how both parameters are, for the most part, constant. At low grid densities between $\sim 200,000$ and $\sim 400,000$ nodes, a small difference can be observed. 


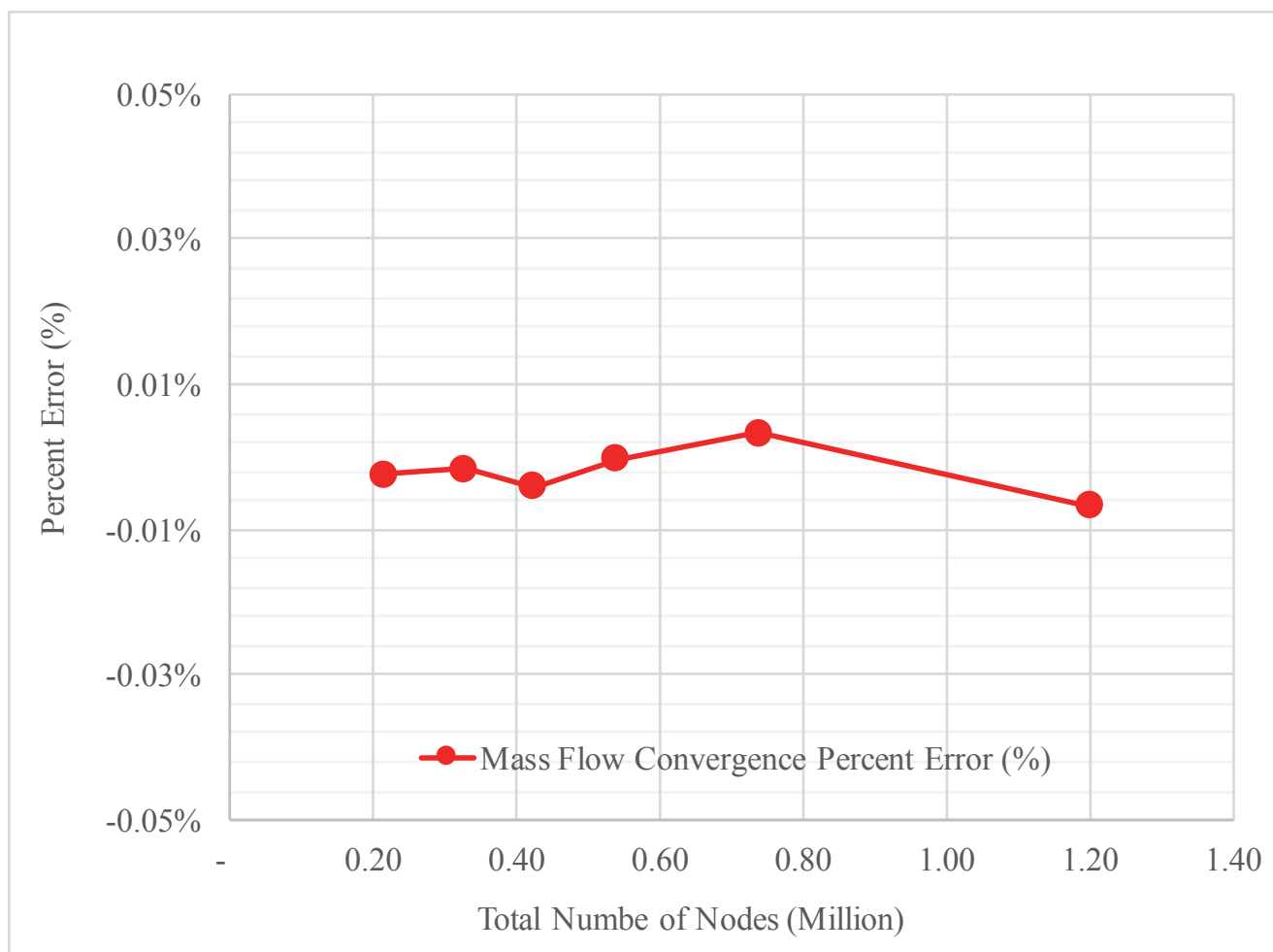

Figure 6-5: Summary of the grid sensitivity analysis. Figure illustrates the percent error corresponding to the mass flow between inlet and specified outlet.

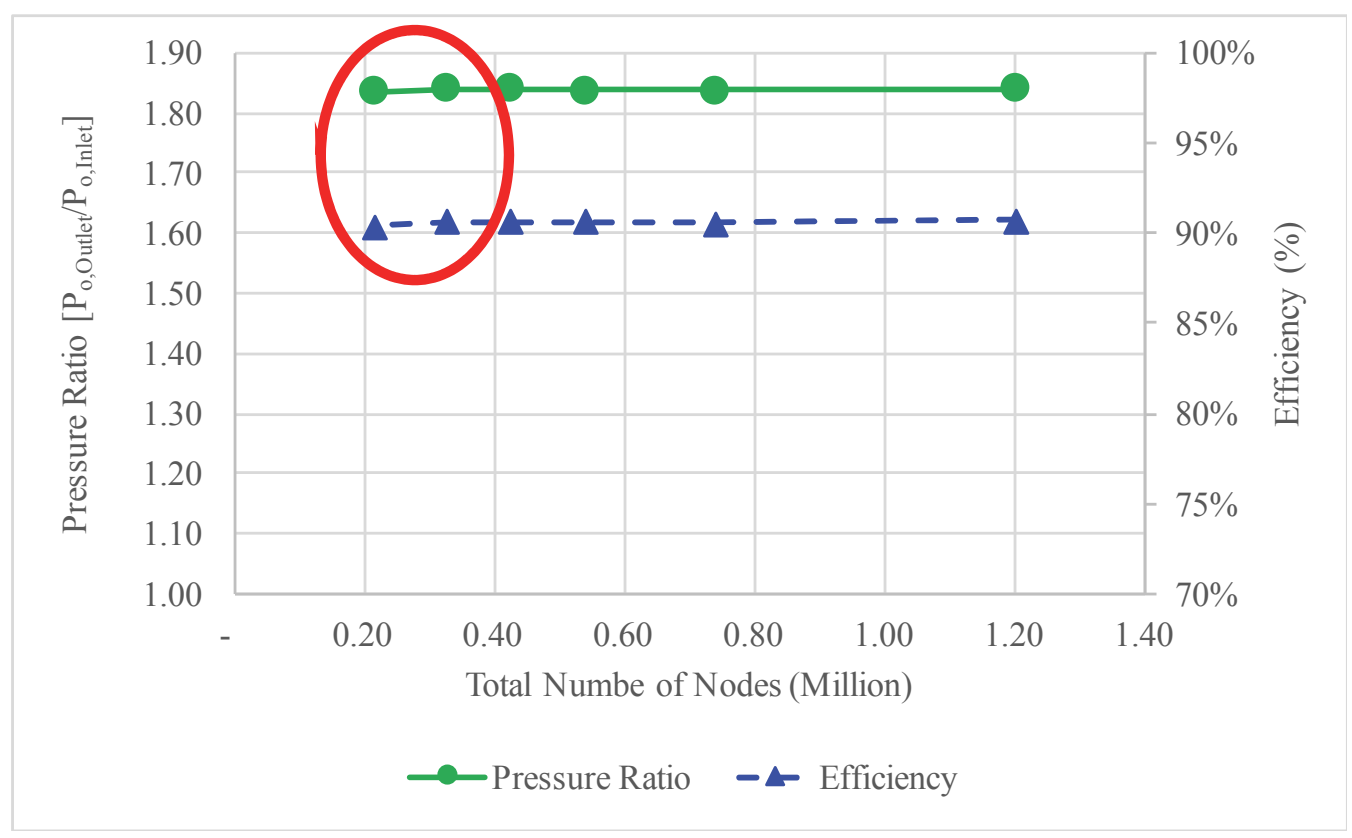

Figure 6-6: Summary of the grid independence for pressure ratio and efficiency. 


\subsection{Aerodynamic Results}

One of the design considerations for this project was to achieve a wide range of operation. This was one of the design goals established by the industrial partner. The main reason for the compressor requires a wide operating range is to help accommodate the possible off-design cases. Recall that the compressor is being designed for a waste heat recovery plant. Therefore, it is not known with certainty how long the heat source will operate at the design point. It was also necessary for the component to have a stable operation within that range. This meant minimizing the changes in efficiency and pressure ratio along the speed line. Figure 6-7 illustrates the design point constant speed curve and efficiency curve corresponding to the final design.

It is important to understand that the design process is iterative in nature. Several geometries were investigated before reaching the final design. The different geometries were evaluated by comparing the achieved aerodynamic values to those desired. Some of the aerodynamic parameters used to guide the design were the De Haller Number and Flow Coefficient, pressure ratio and efficiency. Similarly, some physical geometry values were also investigated including throat length, the impeller outlet width and diffuser outlet. The design aimed to obtain a design which balanced these design considerations. In the next sections, the evaluation of each consideration is described. 


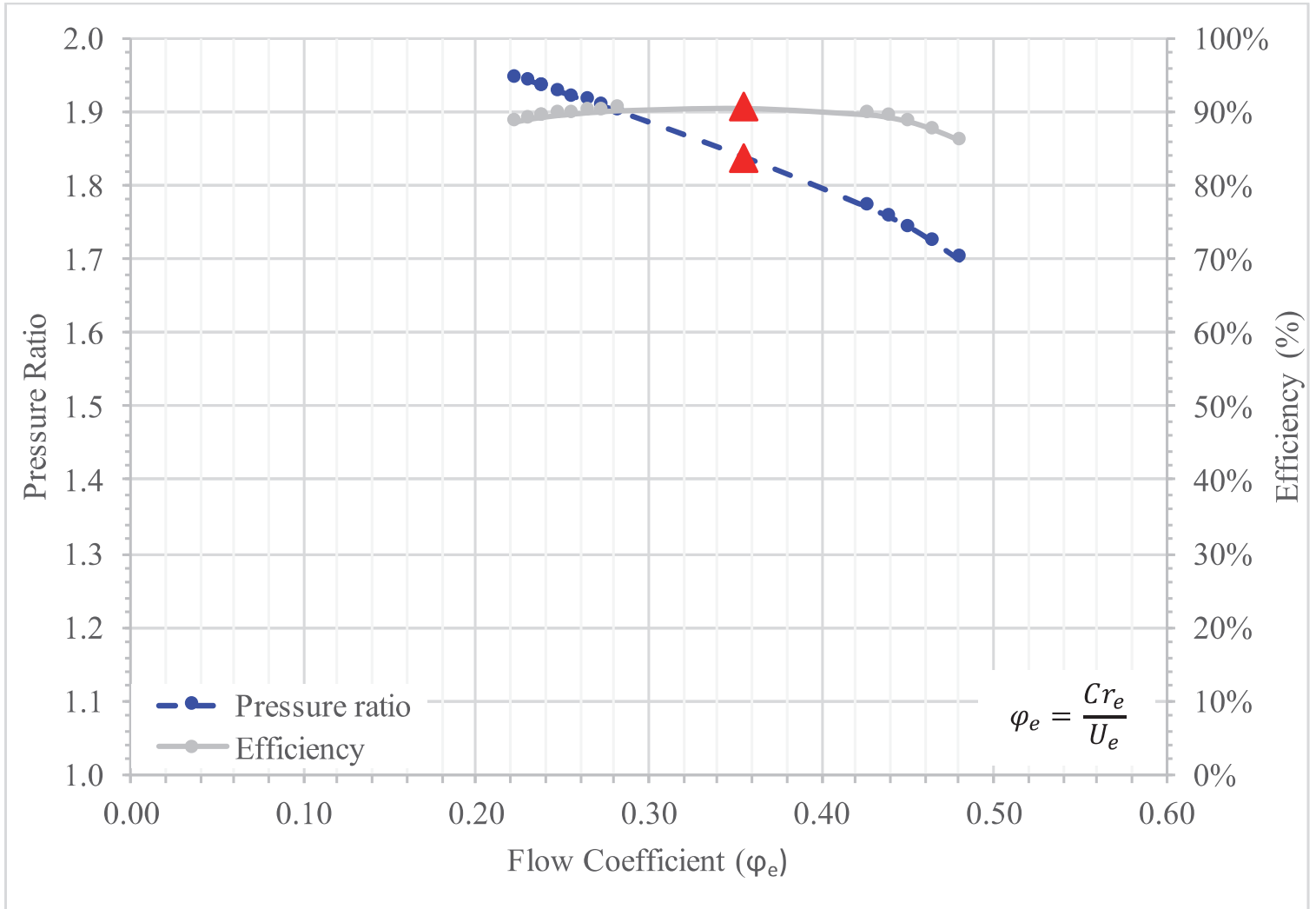

Figure 6-7: Constant speed curve and efficiency curve of the design point rotational speed corresponding to the final preliminary geometry.

There are two y-axes; the blue-dashed line corresponds to the pressure ratio (left y-axis) along the operating line and the grey line corresponds to the efficiency (right $y$ axis). The $\mathrm{x}$-axis corresponds to the Flow Coefficient $\left(\varphi_{\mathrm{e}}\right)$. One can observe that the design point (red triangle) has been shifted to a higher value of $\varphi_{\mathrm{e}}=0.35$, compared to the initial geometry (V2) which was $\varphi_{\mathrm{e}}=0.19$. Similarly, the new design point had a mass flow surge margin (equation 2.9 ) of $35 \%$ versus $2 \%$ for $\mathrm{V} 2$.

The $3 \mathrm{D}$ aerodynamic results presented in this report correspond to Design Point \#2, which is within the supercritical region but behaves as an ideal gas. For this reason, there are values such as the pressure ratio, efficiency or velocity components that do not necessarily correspond to the actual values of Design Point \#1. However, they do provide 
important information regarding the aerodynamic performance of the machine. As it was previously explained (Chapter 2 and 3), the two design points (\#1 and \#2) are partially dynamically similar and the two components would be geometrically similar. In a similar manner, through the application of dimensional analysis one can determine the values of pressure ratio and efficiency corresponding to Design Point \#1. The methodology to obtain such information is presented in Chapter 7. However, from Figure 6-7 one can observe several aspects about the design.

- The design point lies well within the center of the constant speed curve, with a mass flow surge margin of $35 \%$.

- The efficiency of the design point corresponds to the highest along the constant speed curve.

- Both the pressure ratio and the efficiency exhibit trends one would expect of a centrifugal machine.

- The pressure ratio varies by $13 \%$ between the maximum pressure ratio and lowest.

- The efficiency varies by $5 \%$ between maximum and minimum.

These trends are very important as, through similarity, one would expect the design point near the critical point to exhibit the same kind of performance.

CFD is useful tool to obtain an initial aerodynamic analysis and first approximation of the performance. However, there are certain losses that CFD cannot quantify. For example, CFD does not account for parasitic losses: disk friction, recirculation, leakage, etc. For this reason, the efficiency calculated by CFD tends to be higher than what is expected of the actual machine. Despite this overestimation, it is a good first estimate of the performance of the design. 


\subsubsection{De Haller Number}

One of the main parameters used to guide the design of the geometry was the De Haller number. Recall, that the De Haller Number represent the diffusion within the impeller. Section 2.9.1 summarized some of the values recommended in literature. For convenience, Figure 6-8 highlights (yellow region) the minimum recommended range of De Haller Number. Similarly, Figure 6-8 summarizes the different De Haller Numbers, achieved at the design point during the geometry design process. The design process focused on achieving a De Haller Number larger than 0.8. From the same figure, how the first geometries (2-4) were well below the recommended De Haller Numbers. The amount of diffusion within the impeller affects the overall efficiency of the system. As one can see from Figure 6-8 the first three geometries experience the lowest values of De Haller Number. They also experienced the lowest efficiencies, as seen in Figure 6-9. This figure illustrates the different efficiencies (at the design point) obtained with each of the geometries investigated during the design process. It is important to remember diffusion is not the only parameter affecting the efficiency. 


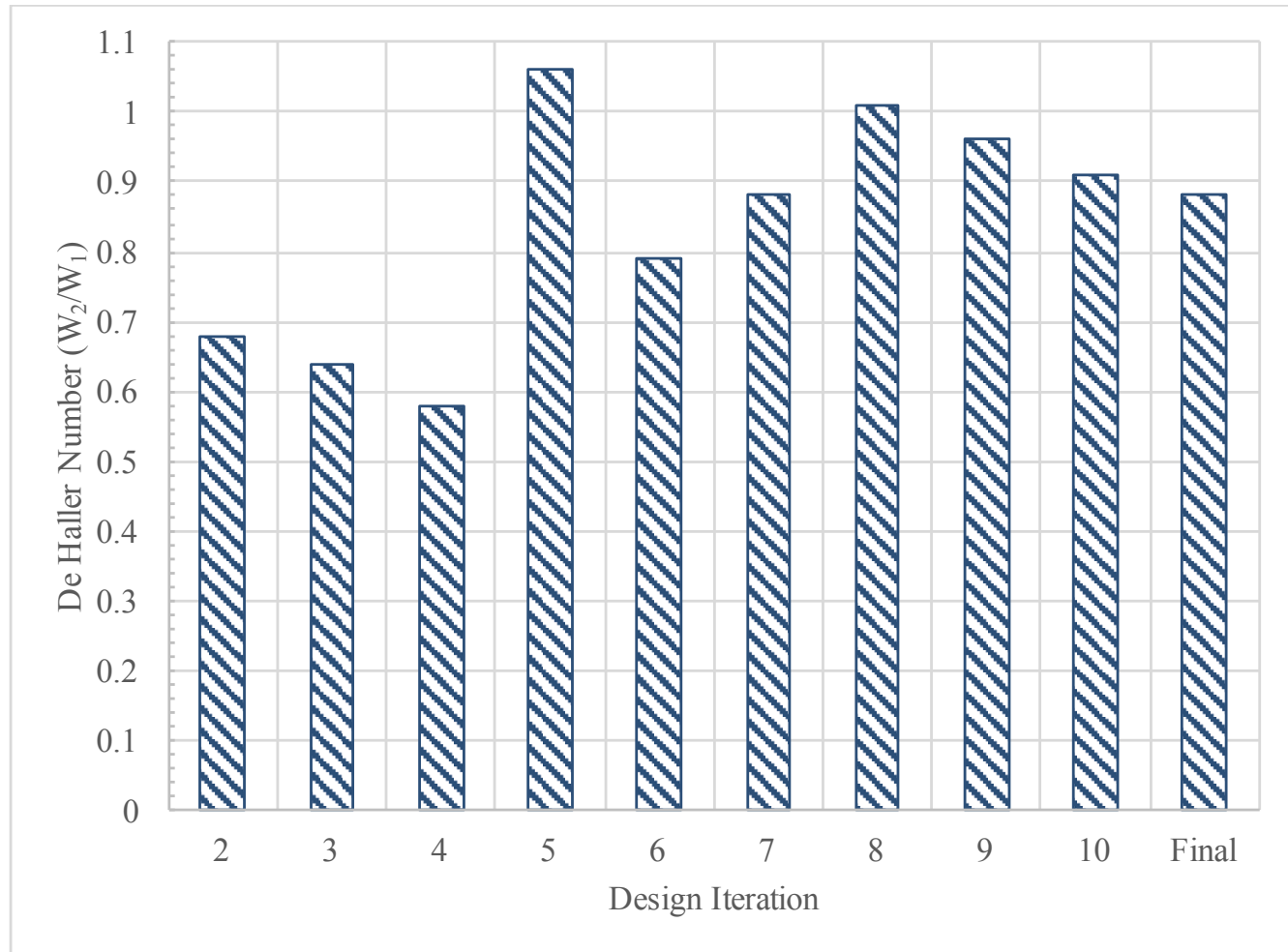

Figure 6-8: Summary of the De Haller Number for each of the different geometries investigated at the design point. The yellow region corresponds to the different min values of De Haller recommended by different authors.

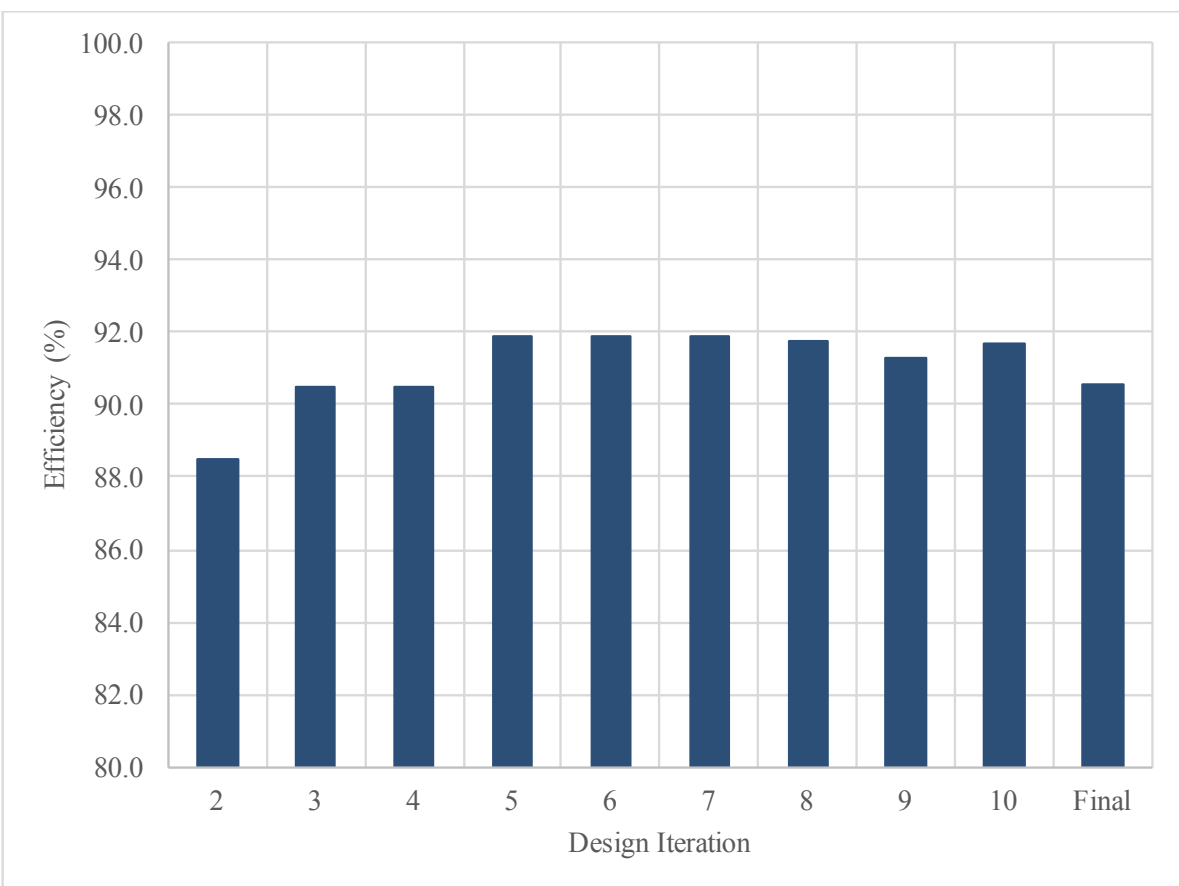

Figure 6-9: Geometry investigation - estimate of the component's efficiency at the design point. 
The De Haller Number influenced the geometry design at different regions of the impeller. The first region was the impeller outlet width (b). Figure 6-10 summarizes the changes in impeller outlet width (b). It is important to mention that the outlet width was also influenced by the flow behaviour in the diffuser, as will be shown in Section 6.6.4. Therefore, a design compromise had to be made between the effects of impeller width outlet (b) on the De Haller Number and the diffuser flow.

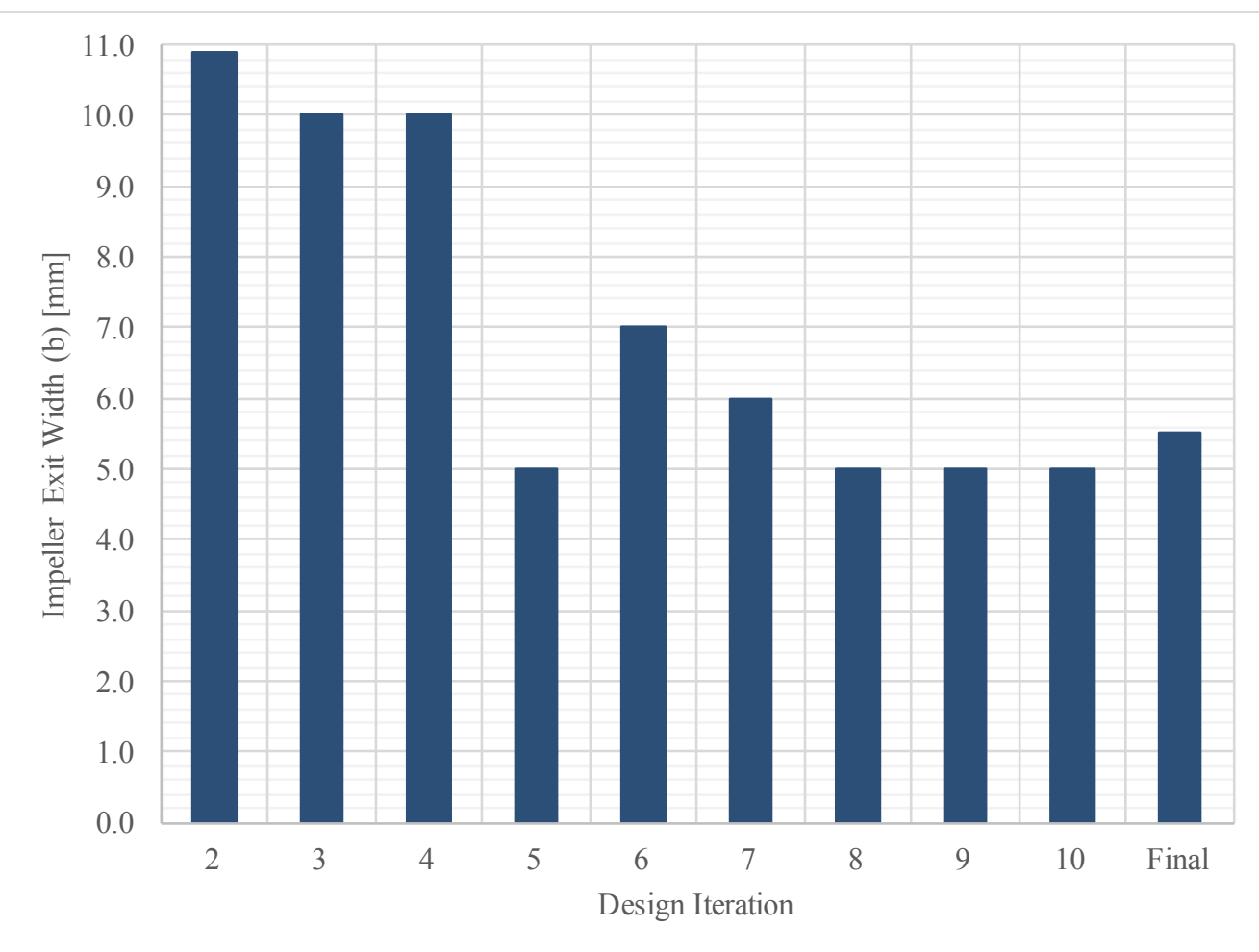

Figure 6-10: Impeller outlet width (b) corresponding to each of the investigated geometries.

The next regions, where the De Haller Number influenced the geometry were the impeller inlet hub radius and tip radius. As expected, by varying the impeller inlet and outlet areas, one influences the components of relative velocity. 
The De Haller Number is not only affected by the inlet and outlet areas. The flow conditions at the inlet are also affected by the geometry. Therefore, the shape of the inducer, as well as the throat area affected the values of De Haller. Figure 6-11 summarizes the blade-to-blade throat distance $(\mathrm{mm})$ at the hub and shroud. The final preliminary design achieveed the largest blade-to-blade throat distance (mm). This is important as the larger distances relate to larger throat areas. The throat area is important as it influences the flow velocity as well as the region at which the machine becomes choked. As it was discussed in Section 4.2.1 the throat is of great interest when operating near the critical point, as the flow velocity in this region can influence the two-phase flow. However, due to the simulation constraints this aspect of the design need further analysis.

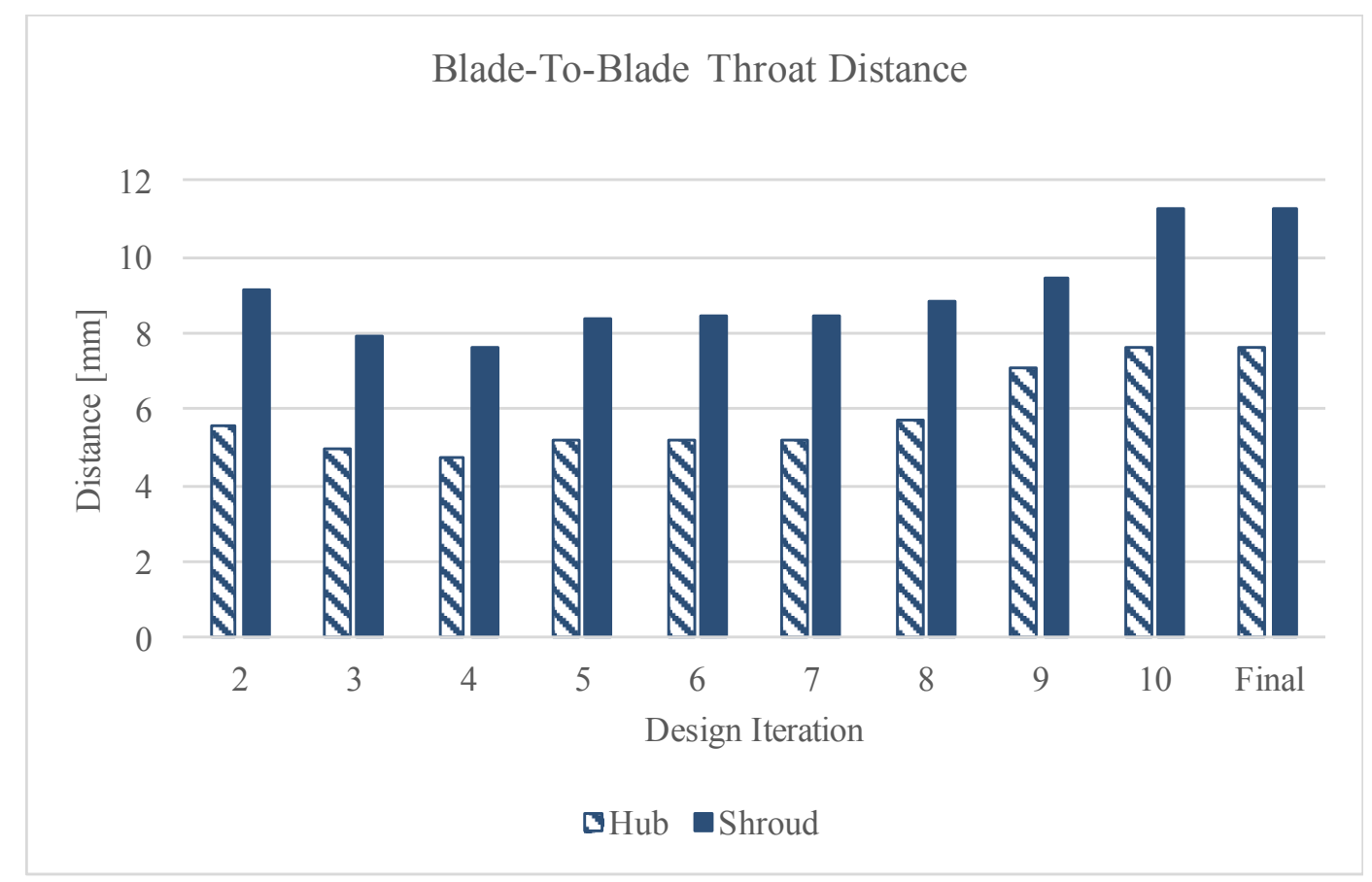

Figure 6-11: Blade-to-blade throat distance $(\mathrm{mm})$ corresponding to each of the investigated geometries. 
Figure 6-7 presented the operating range of the compressor at the design point rotational speed. The compressor experienced small changes in pressure ratio and efficiency, suggesting a wide stable operating region. When the De Haller Number was analyzed for each operating point along the constant speed curve, the De Haller Number remained within the literature recommended values, as shown in Figure 6-12. The yellow region once again summarizes the minimum values of De Haller Number recommended by several authors.

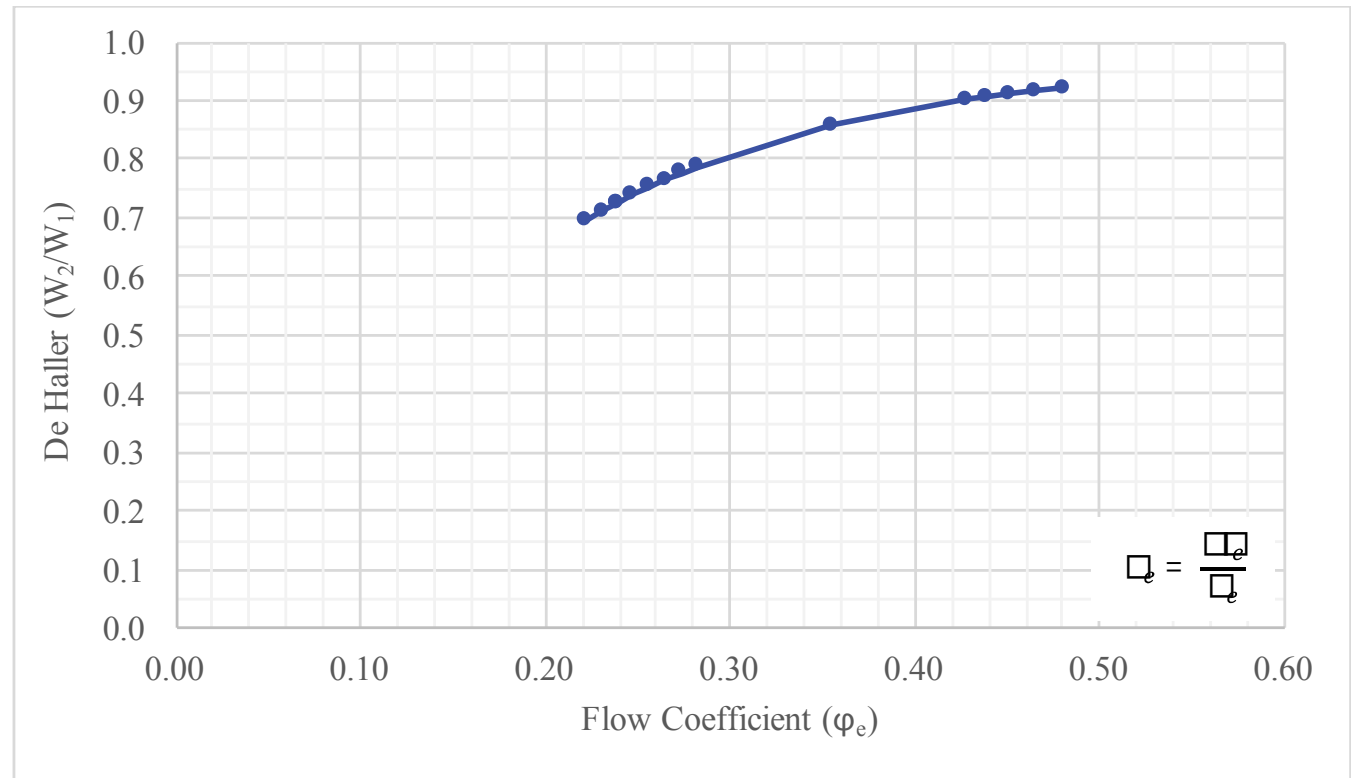

Figure 6-12: De Haller values corresponding to each operating point along the design point constant speed curve.

\subsubsection{Flow Coefficient $\left(\varphi_{e}\right)$}

Another important parameter used to guide the design process was the Flow Coefficient $\left(\varphi_{\mathrm{e}}\right)$. As explained in Section 2.6, Eckardt data suggestes that well-designed compressors approach the stall region at about $\varphi_{e} \approx 0.2$ to 0.25 and choke region at 
about $\varphi_{e}=0.55$ to 0.6 . The CFD results show that the current impeller design experiences a similar trend as shown in Figure 6-13. One can observe that the speed line ranges from $\varphi_{e} \approx 0.2$ to 0.54 . In a similar manner, the remaining speed lines making up the compressor performance map followed the same trend for Flow Coefficient $\varphi_{e}$.

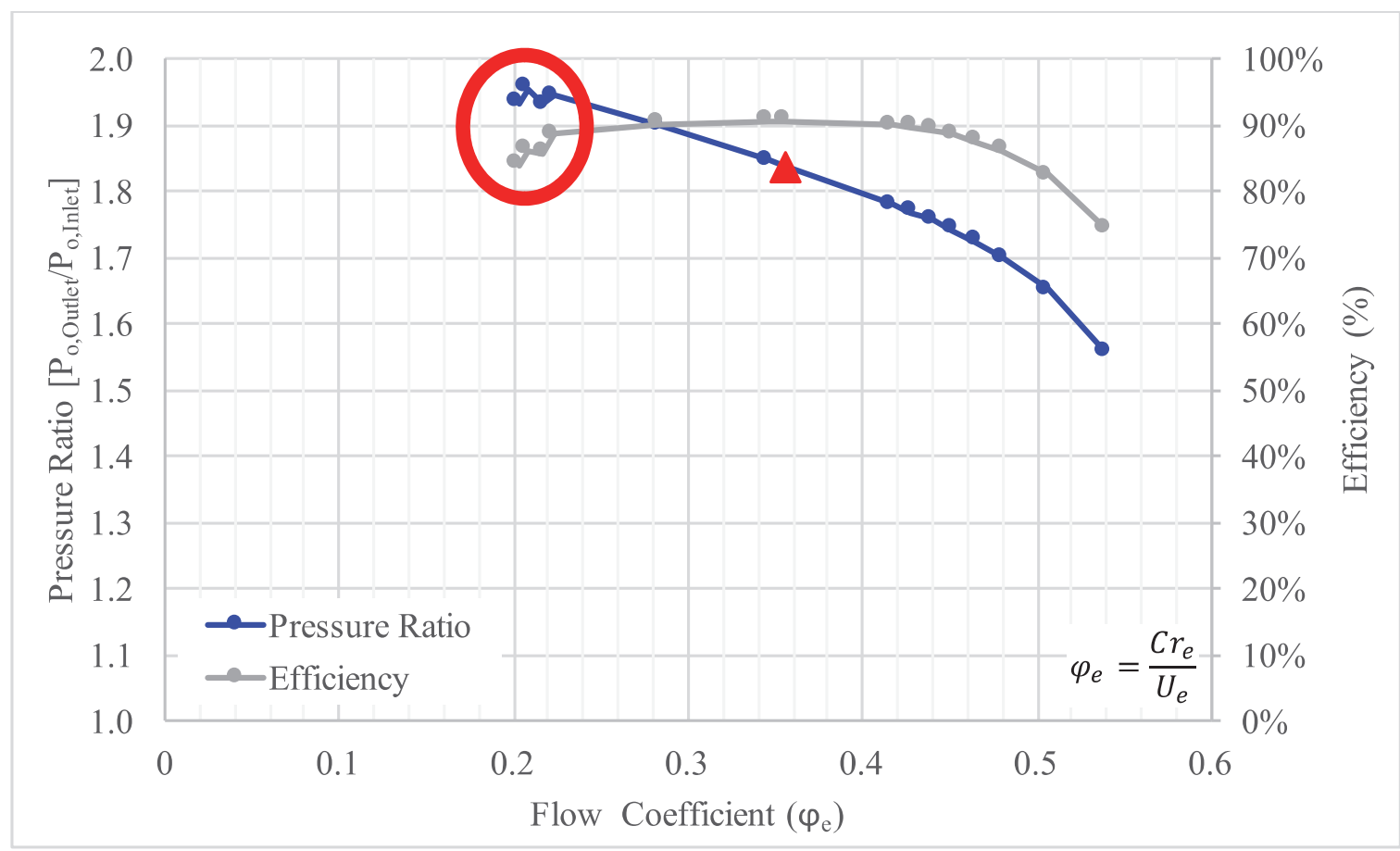

Figure 6-13: Constant speed curve and efficiency curve of the design point rotational speed corresponding to the final preliminary geometry. The red circle highlights the poor convergence near the stall region.

Section 6.2 presented the mass flow percent error and how this was used to further evaluate the CFD output data. It was found that the operating points near the surge and choke region would exhibit poor convergence as well as $\epsilon_{m}>0.1 \%$. In a similar manner, for the same values with poor convergence, the value of Flow Coefficient $\left(\varphi_{\mathrm{e}}\right)$ were found to be within the limiting values suggested by the Eckardt data. 
Figure 6-13 demonstrates that the points showing some discrepancies are located at values of $\varphi_{e} \sim 0.2$ (red circle). Similarly, one can observe a sharper decline in efficiency for $\varphi_{e} \geq 0.5$.

The behavior of the flow can also be seen through the 3D graphical representation. The following set of figures illustrate the component of relative velocity at three different span-wise location: $10 \%, 50 \%$ and $90 \%$ on the hub, mean and shroud respectively. Each span-wise location figure is presented for three different operating points; Design Point (Figure 6-14 to Figure 6-16), Stall Region (Figure 6-17 to Figure 6-19), and Choke Region (Figure 6-20 to Figure 6-22).

The main take-away from these figures is that the flow shows the behavior one would expect as predicted by the Flow Coefficient $\left(\varphi_{e}\right)$. Near the "surge" region there is a high degree of flow separation indicating the possibility of stall. Near the choke region, it was found that the compressor does not physically choke, but there is a large presence of vorticity and flow separation at the shroud. At the design point, one can observe a well-behaved velocity flow field. It is important to note that all nine figures have the same scale. This was chosen to illustrate the different velocity at each of the three operating points. At a first look, it may appear as if the flow in the design point experiences a degree of flow separation and therefore stall, however, this is not the case. 


\section{Design Point Region}

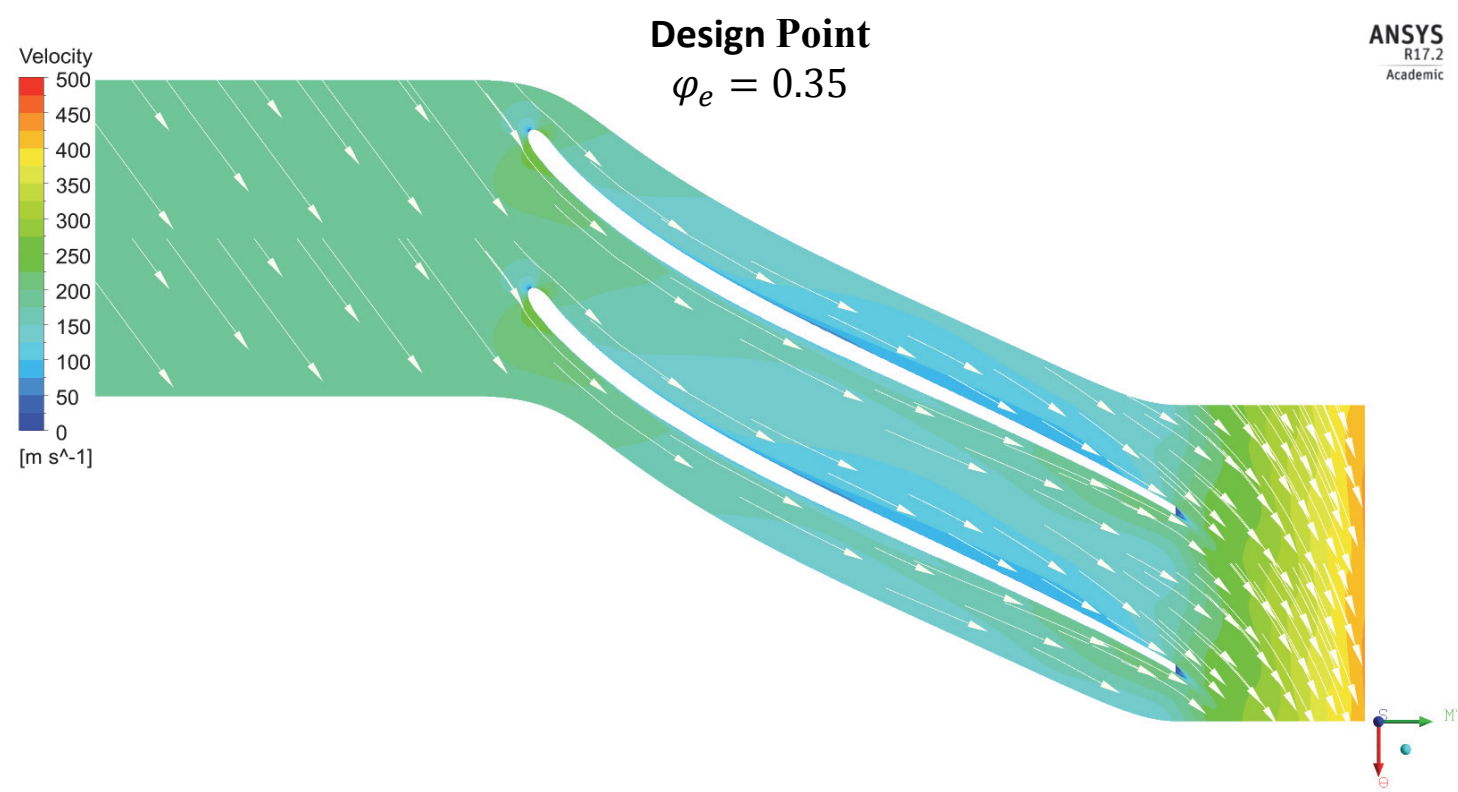

Figure 6-14: Design Point Region- Contour of relative velocity $[\mathrm{m} / \mathrm{s}]$ at $10 \%$ span. The mass flow at this operating point is $42.903 \mathrm{~kg} / \mathrm{s}\left(\varphi_{e}=0.35\right)$.

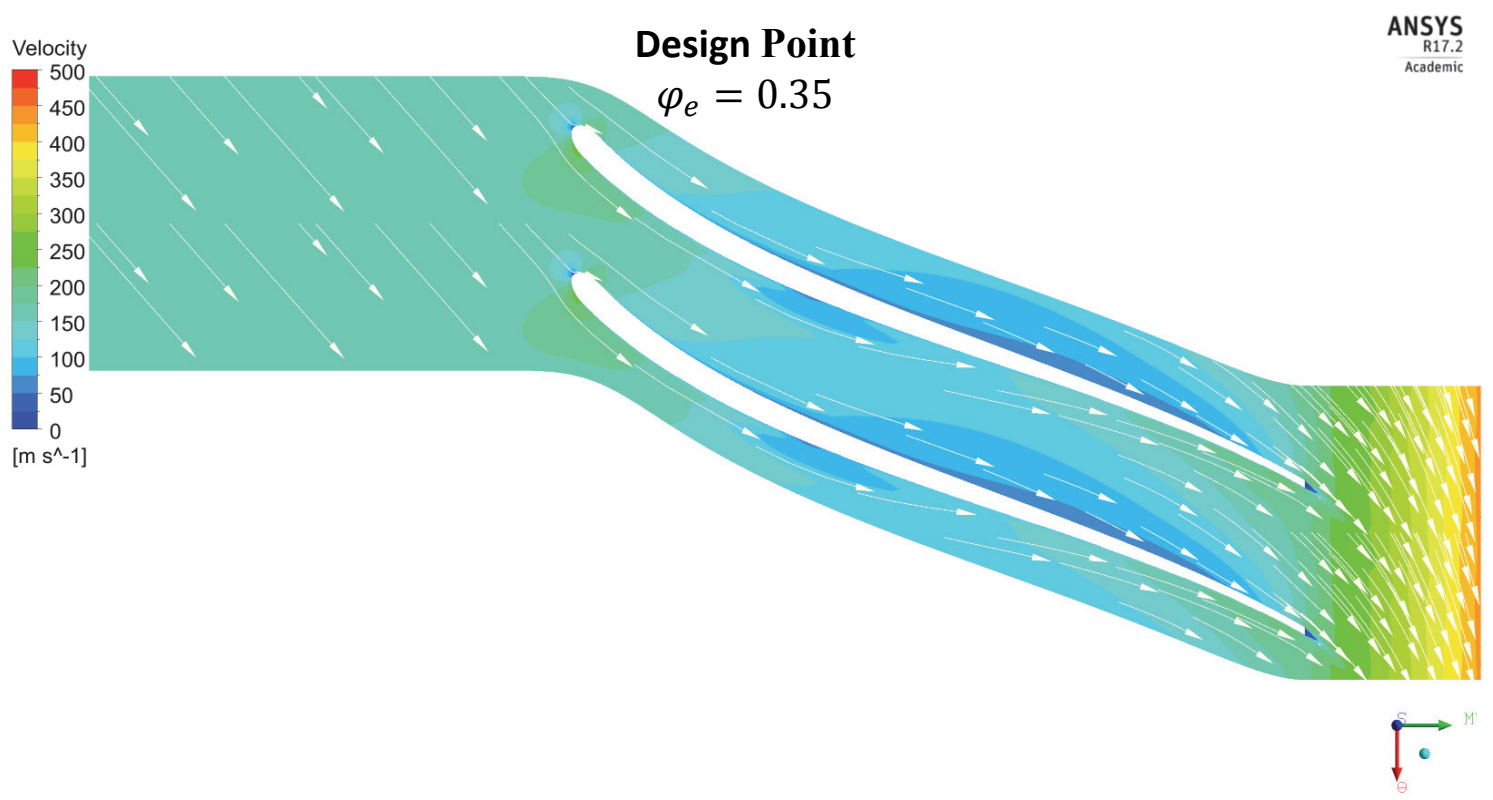

Figure 6-15: Design Point Region - Contour of relative velocity $[\mathrm{m} / \mathrm{s}]$ at $50 \%$ span. The mass flow at this operating point is $42.903 \mathrm{~kg} / \mathrm{s}\left(\varphi_{e}=0.35\right)$. 


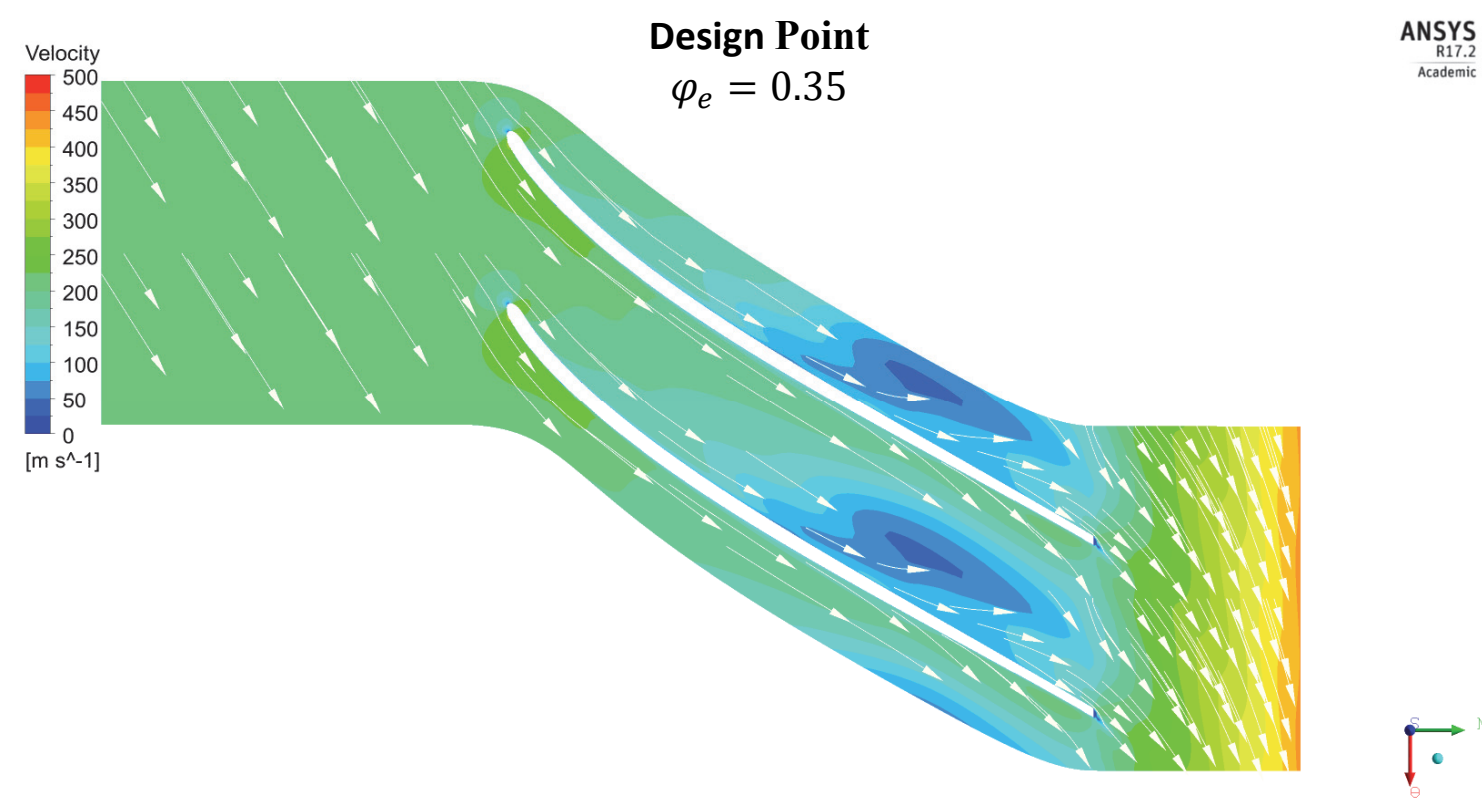

Figure 6-16: Design Point Region - Contour of relative velocity $[\mathrm{m} / \mathrm{s}]$ at $90 \%$ span. The mass flow at this operating point is $42.903 \mathrm{~kg} / \mathrm{s}\left(\varphi_{e}=0.35\right)$.

\section{Stall Region}

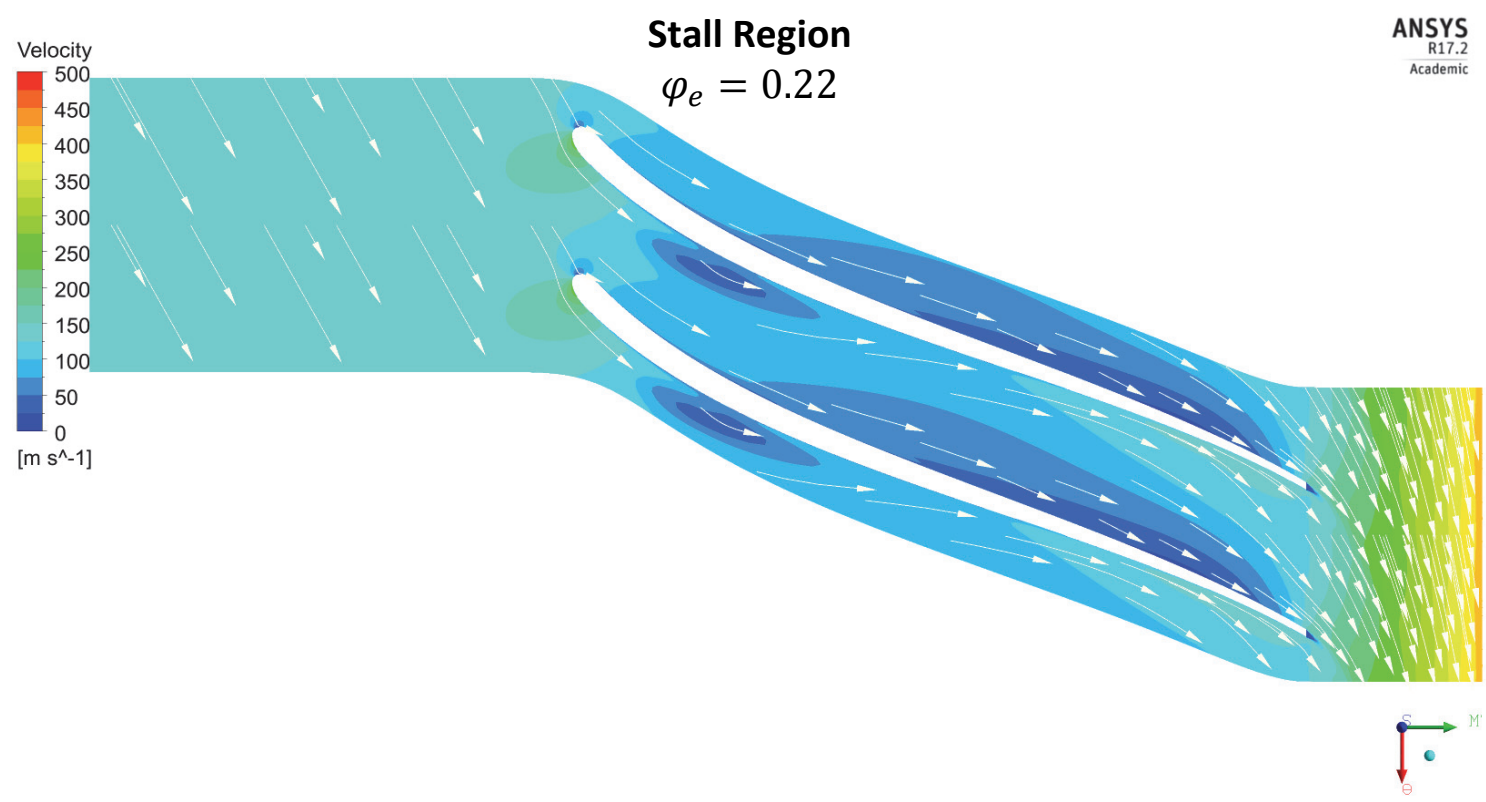

Figure 6-17: Stall Region - Contour of relative velocity $[\mathrm{m} / \mathrm{s}]$ at $10 \%$ span. The mass flow at this operating point is $28 \mathrm{~kg} / \mathrm{s}\left(\varphi_{e}=0.22\right.$. $)$. 


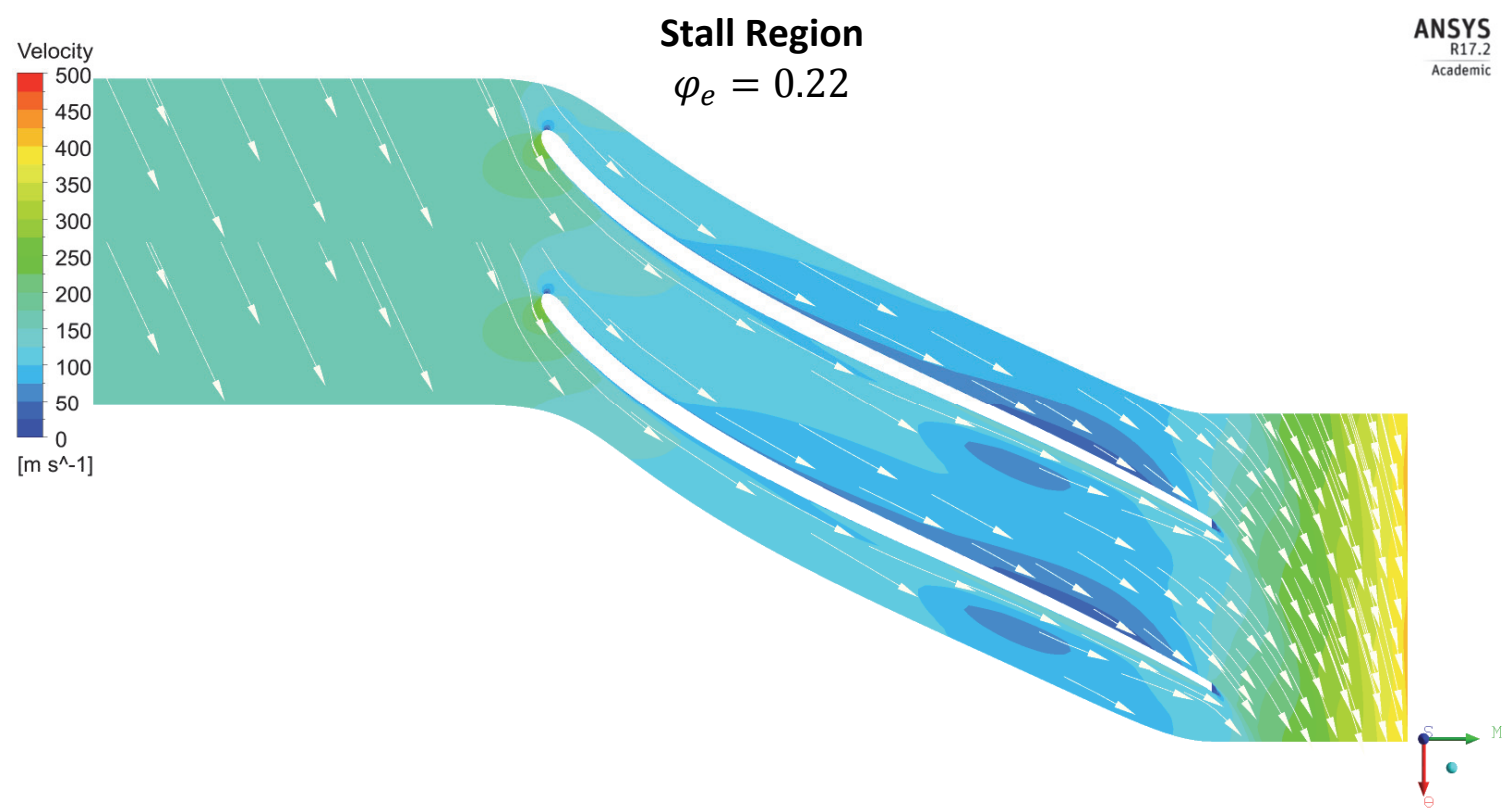

Figure 6-18: Stall Region - Contour of relative velocity $[\mathrm{m} / \mathrm{s}]$ at $50 \%$ span. The mass flow at this operating point is $28 \mathrm{~kg} / \mathrm{s}\left(\varphi_{e}=0.22\right)$.

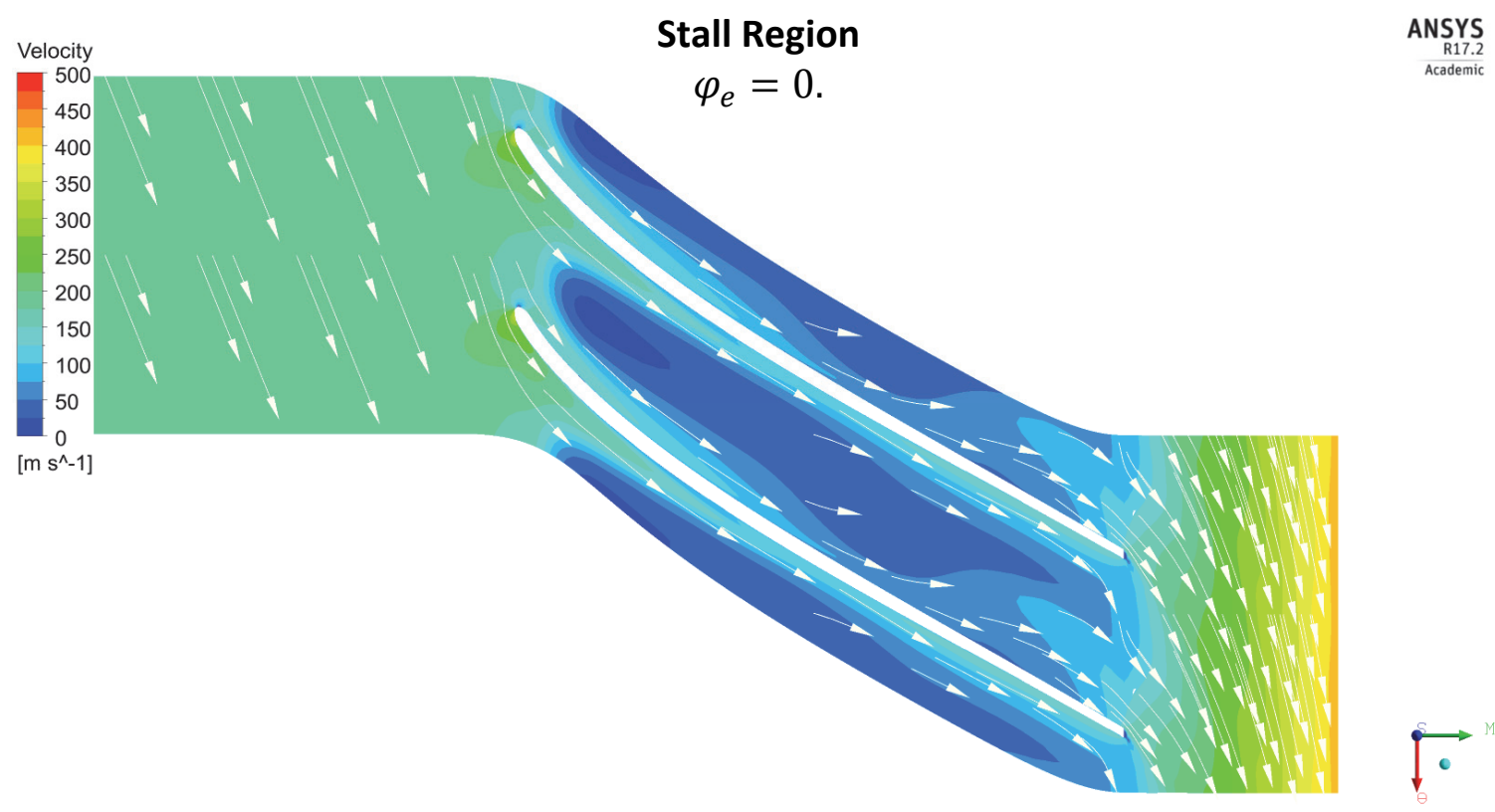

Figure 6-19: Stall Region - Contour of relative velocity [m/s] at 90\% span. The mass flow at this operating point is $28 \mathrm{~kg} / \mathrm{s}\left(\varphi_{e}=0.22\right)$. 


\section{Choke Region}

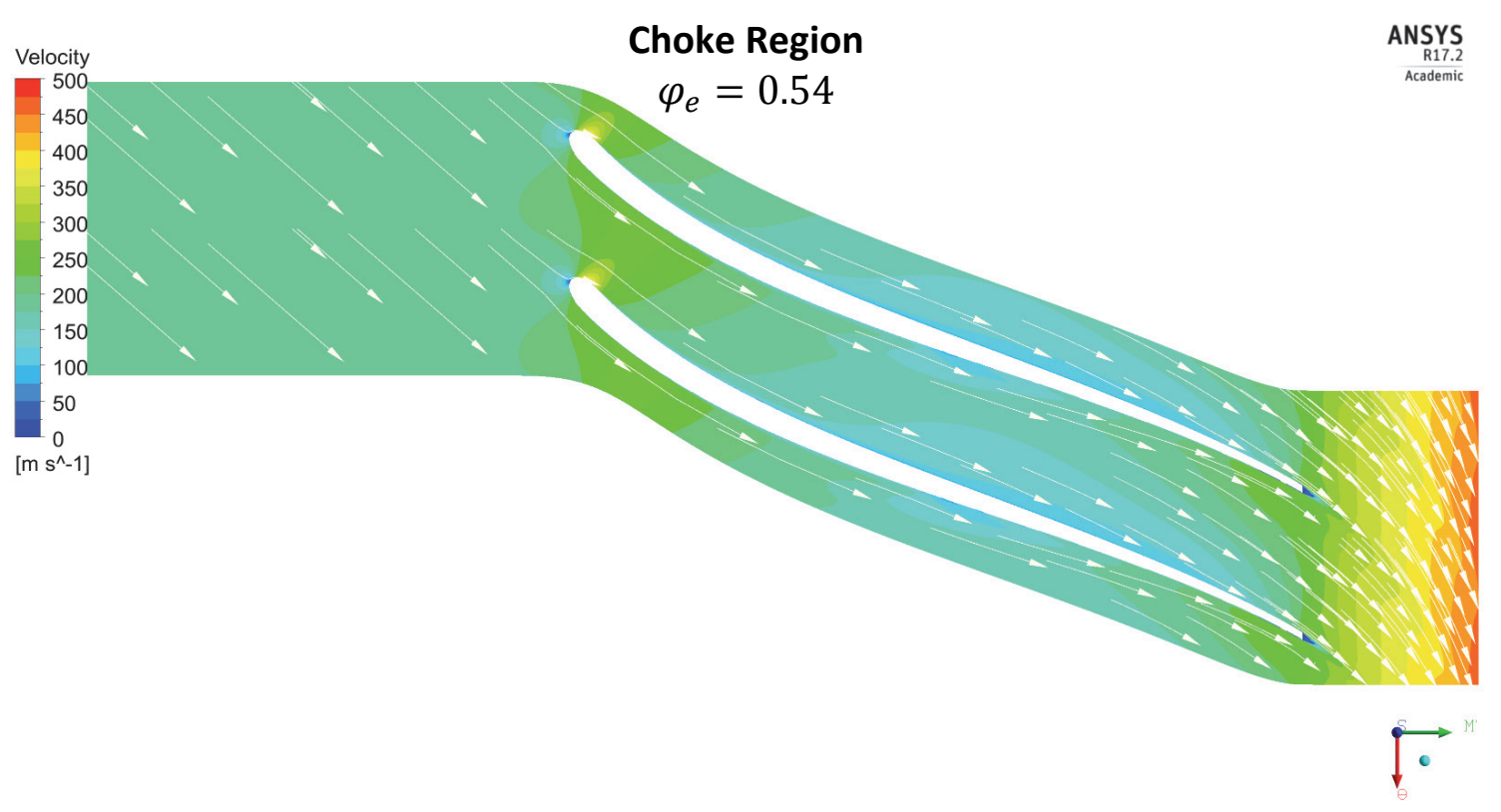

Figure 6-20: Choke Region - Contour of relative velocity $[\mathrm{m} / \mathrm{s}]$ at $10 \%$ span. The mass flow at this operating point is $54 \mathrm{~kg} / \mathrm{s}\left(\varphi_{e}=0.54\right)$.

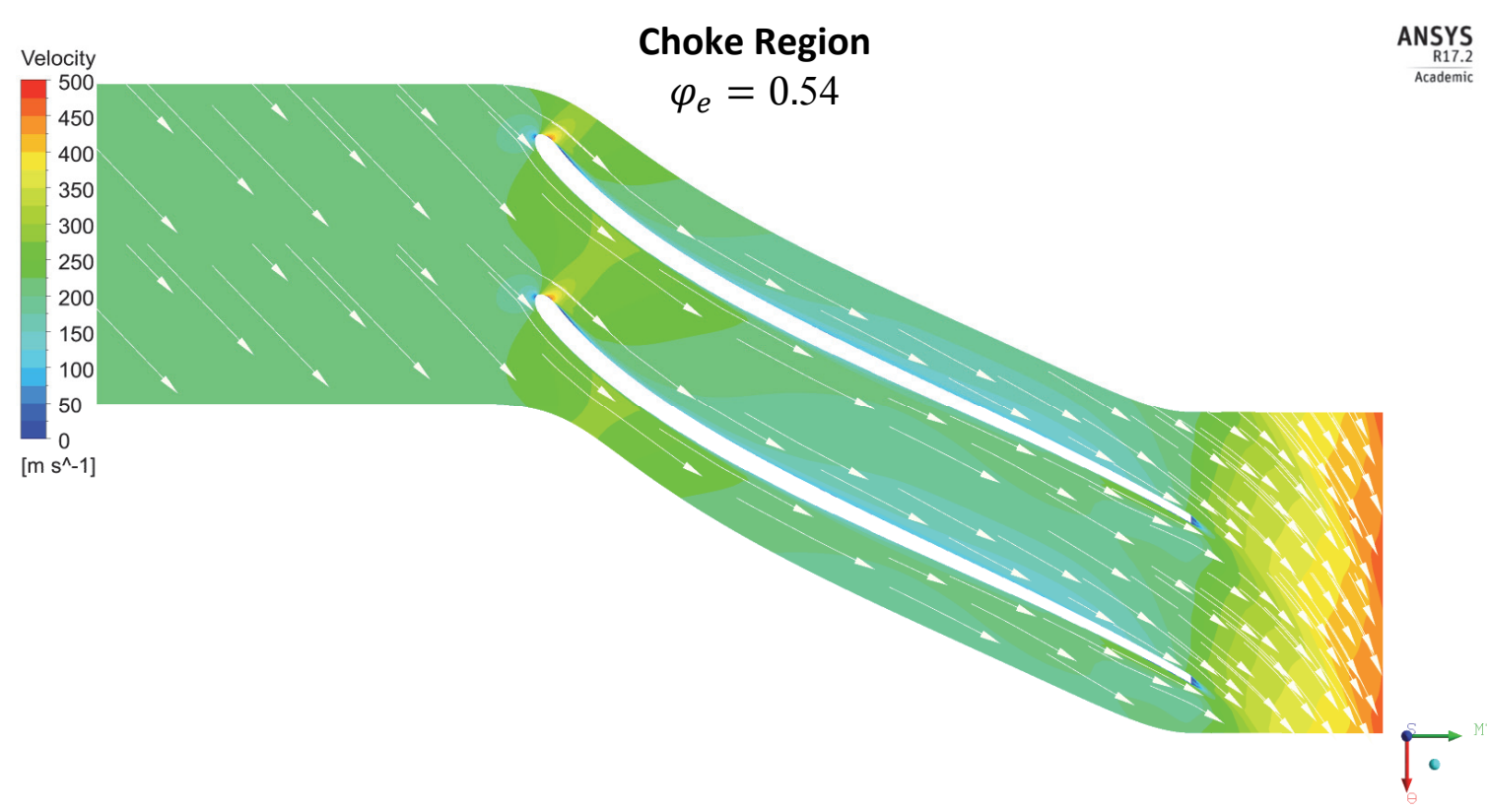

Figure 6-21: Choke Region - Contour of relative velocity [m/s] at 50\% span. The mass flow at this operating point is $54 \mathrm{~kg} / \mathrm{s}\left(\varphi_{e}=0.54\right)$. 


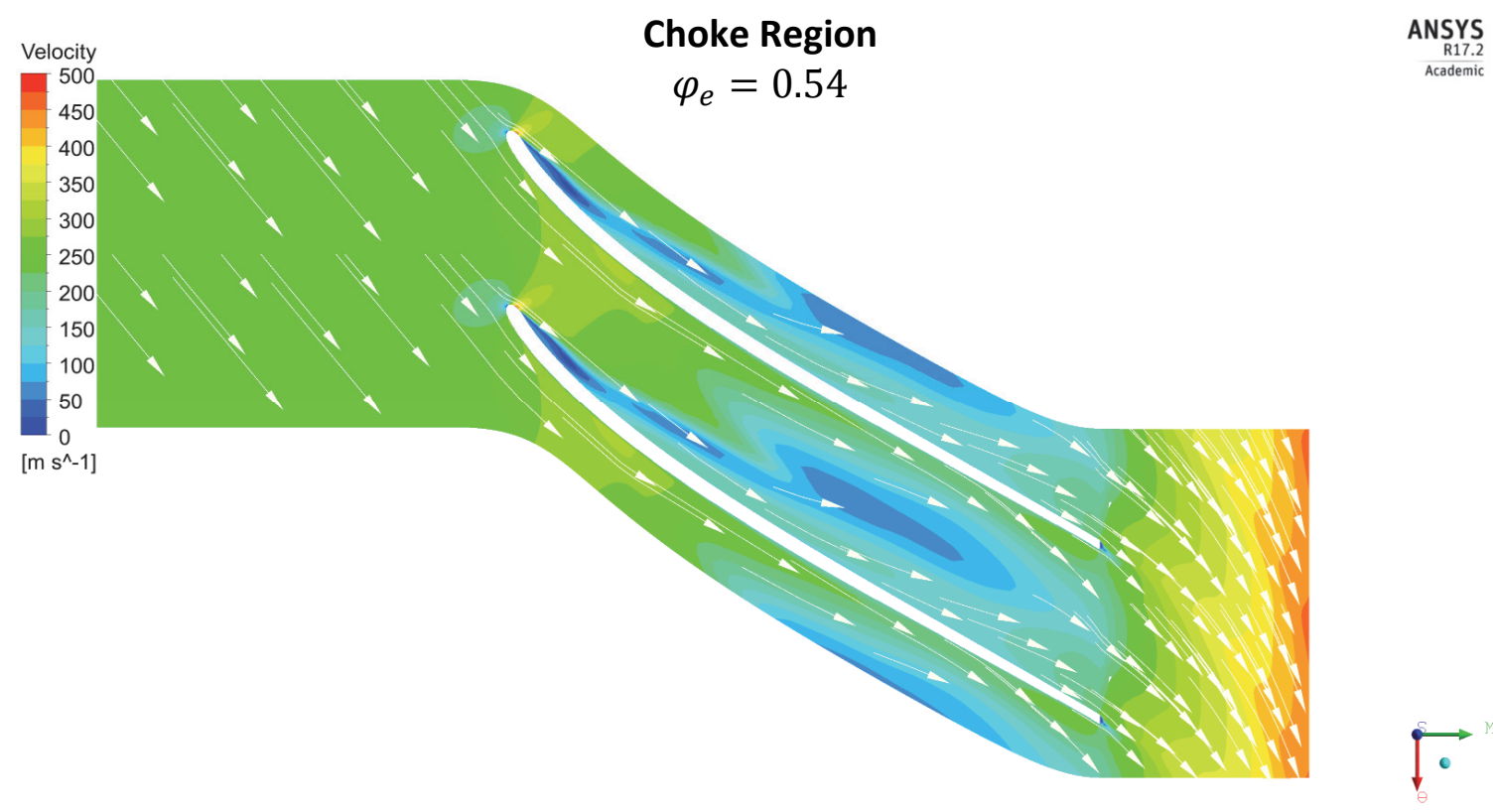

Figure 6-22: Choke Region - Contour of relative velocity $[\mathrm{m} / \mathrm{s}]$ at $90 \%$ span. The mass flow at this operating point is $54 \mathrm{~kg} / \mathrm{s}\left(\varphi_{e}=0.54\right)$.

\subsubsection{Diffuser Design}

The diffuser is the static component that is attached to the outlet of the impeller. The diffuser is responsible for reducing the kinetic energy of the flow and raising the static pressure of the fluid. Therefore, it is necessary that the diffuser minimizes the losses, otherwise this will be reflected in the efficiency of the system and most importantly in the outlet pressure. Figure 6-23 plots the recovery pressure (Cp) and the loss coefficient (K). Figure 6-24 plots the Impeller and Impeller + Diffuser Pressure Ratio. Both figures summarize the values obtained at the design point corresponding to each of the geometries investigated during the design process. 


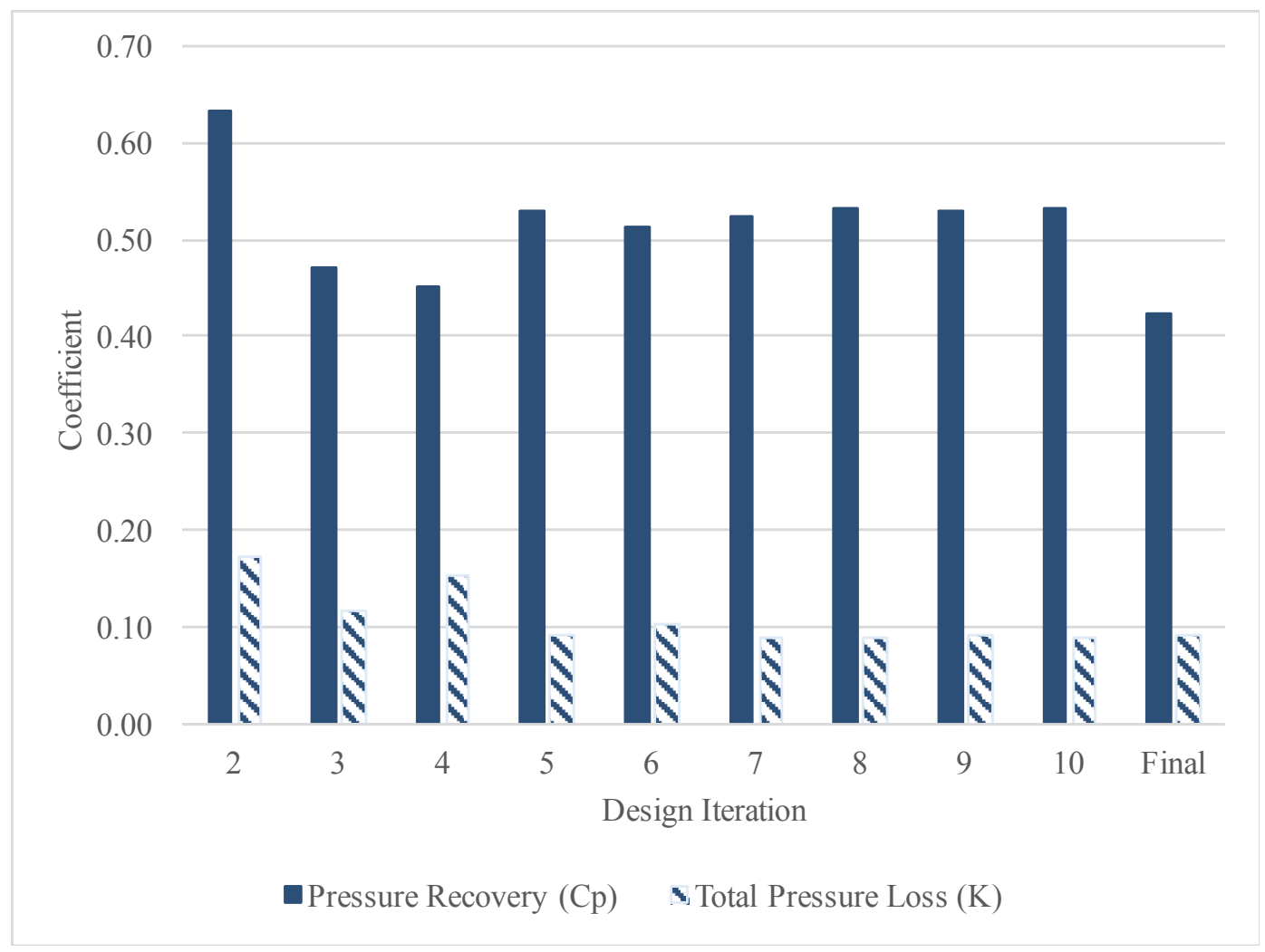

Figure 6-23: Summary of the recovery pressure $(\mathrm{Cp})$ and the loss coefficient $(\mathrm{K})$ corresponding to the design point at each of the investigated geometries.

It is possible to see from Figure 6-23 that the final preliminary geometry achieved a pressure recovery of $\sim 42 \%$. According to Japikse and Baines (1997) typical industrial diffusers have a recovery pressure of about $30 \%$ to $40 \%$. Therefore, the preliminary design satisfies this industrial trend.

The loss coefficient of the final geometry is found to be about $\sim 9 \%$. From Aungier (2002), pinch diffusers commonly experience loss coefficients ranging from $\sim 10 \%$ to $\sim 35 \%$. Therefore, the preliminary design satisfies common industrial loss coefficients. 


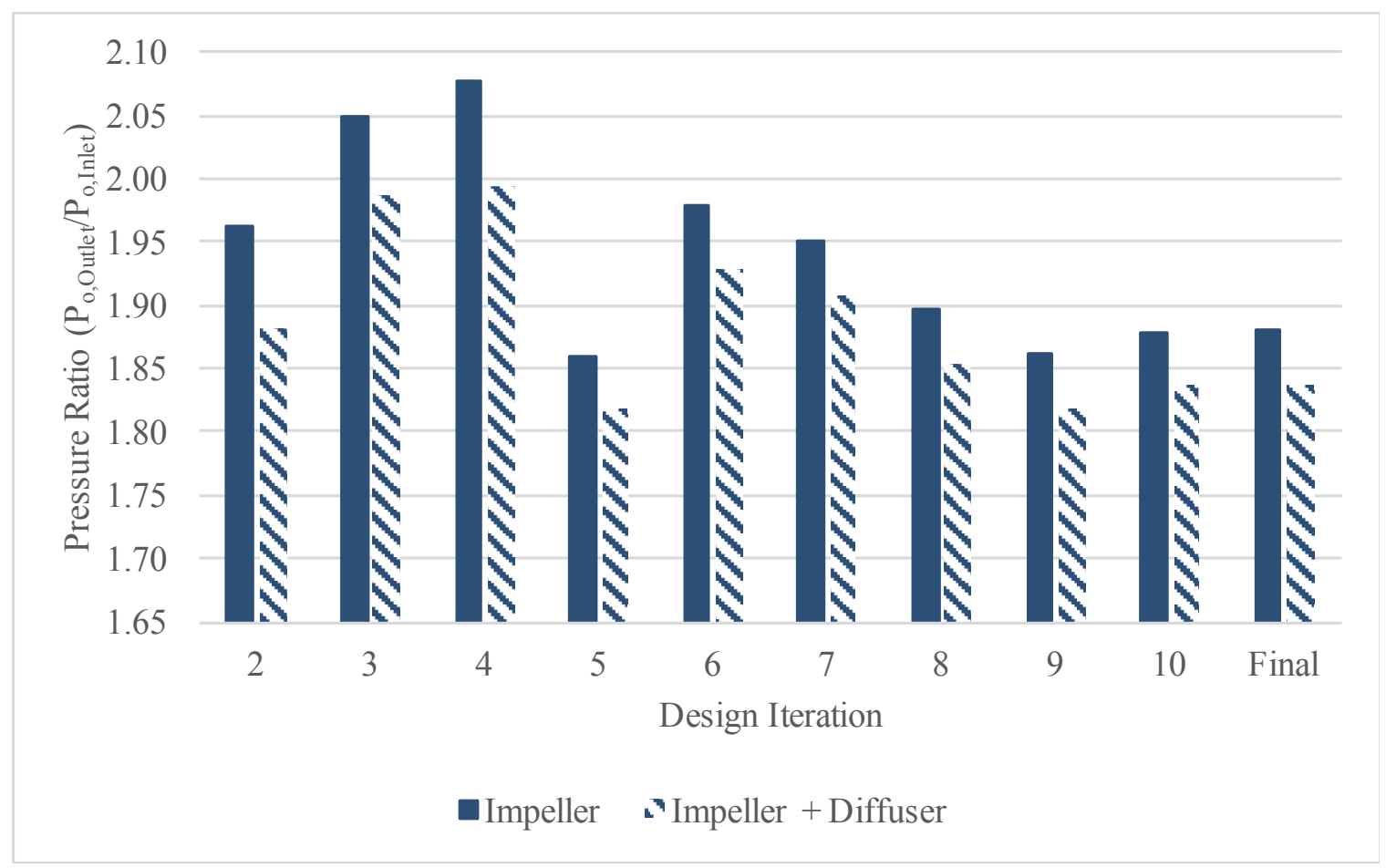

Figure 6-24: Summary of the impeller pressure ratio and the impeller + diffuser pressure ratio corresponding to the design point at each of the investigated geometries.

Figure 6-24 illustrates the variation of pressure ratio. Despite the decrease in pressure ratio between the initial and final geometries, it was found that the final geometry still achieved the desired pressure rise as will be demonstrated in Chapter 7.

It was mentioned in Section 6.6.1 that the impeller outlet width (b) highly influenced the geometry design. As previously mentioned, this affects the De Haller Number. However, it also affects the flow inside the diffuser. Figure 6-23 demonstrates that the initial geometry (V2) had a high-pressure recovery of about $\sim 60 \%$. However, based on the CFD flow results shown in Figure 6-25, large flow separation is observed. taking place inside the diffuser. It is possible to see that the flow separation in the diffuser is a result of the wake coming out of the impeller. The large vorticity leaving the impeller along with the large diffusion taking place inside the diffuser were causing the flow to 
separate. The flow separation inside the diffuser was the guiding factor towards reducing the inlet width of the diffuser and eventually having a "pinched" diffuser.
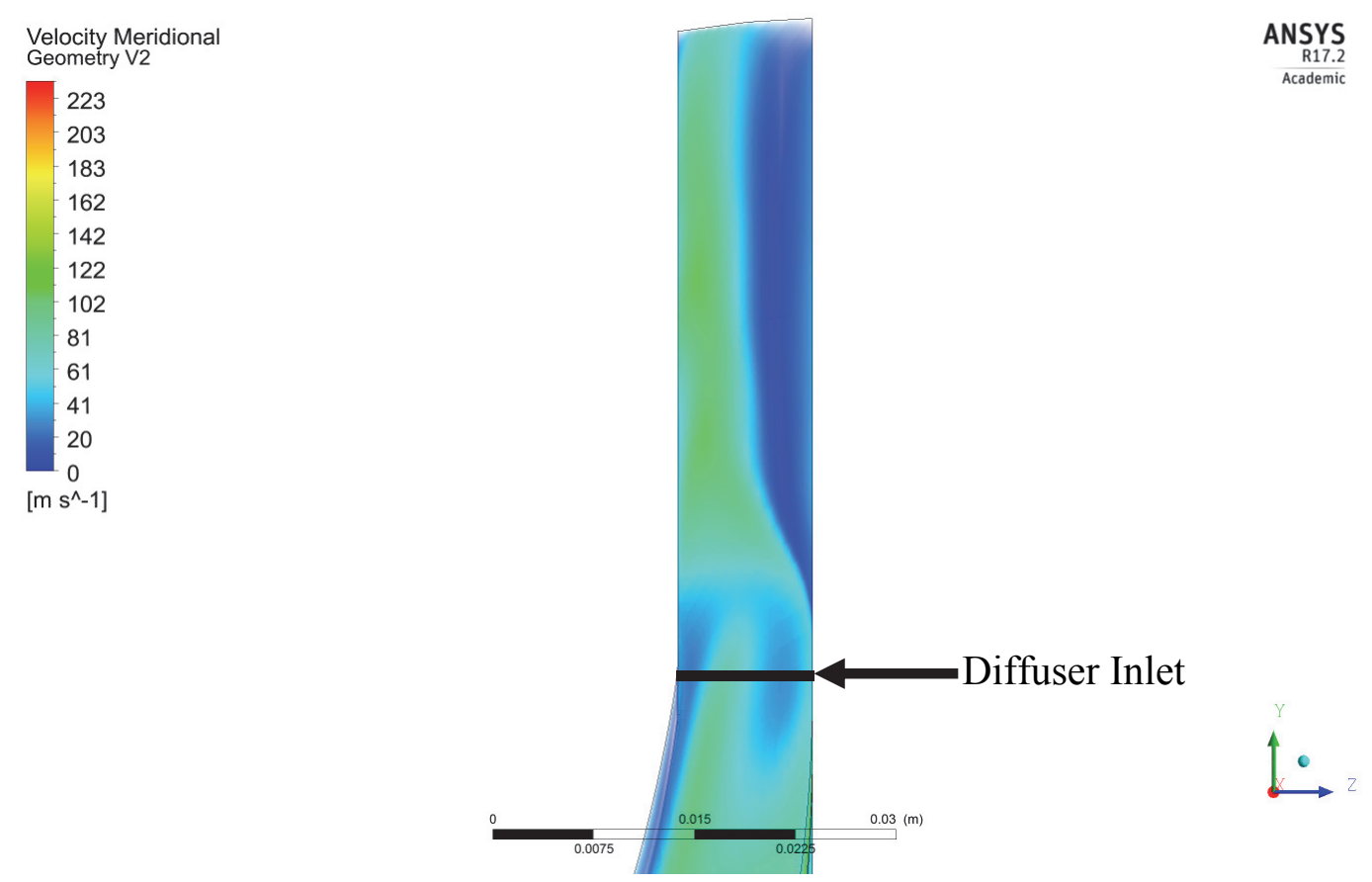

Figure 6-25: Meridional velocity [m/s] inside the diffuser corresponding to the initial geometry (V2).

Figure 6-26 summarize the pressure recovery and loss coefficient of the final geometry. The figure plots the coefficients corresponding to each operating point of the design point constant speed curve. As the mass flow decreased the pressure recovery increased to about $\sim 49 \%$. However, as mass flow increased the pressure recovery decreased to $\sim 25 \%$. The loss coefficient remained relatively stable along the constant speed curve. Figure 6-27 illustrates the diffuser's meridional velocity of the final geometry. 


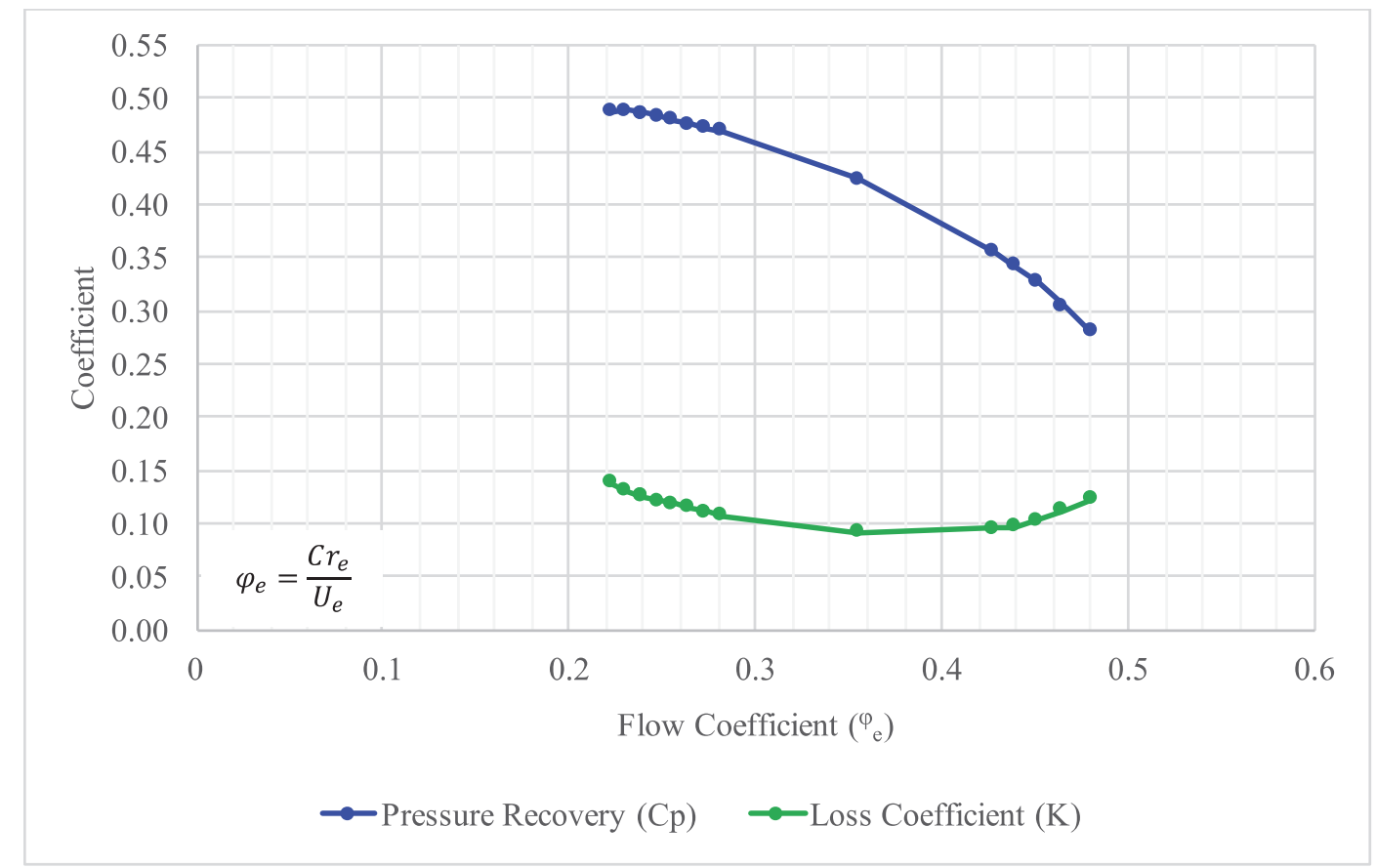

Figure 6-26: Summary of the pressure recovery $(\mathrm{Cp})$ and loss coefficient $(\mathrm{K})$ of the final geometry. Values correspond to the design point constant speed curve.
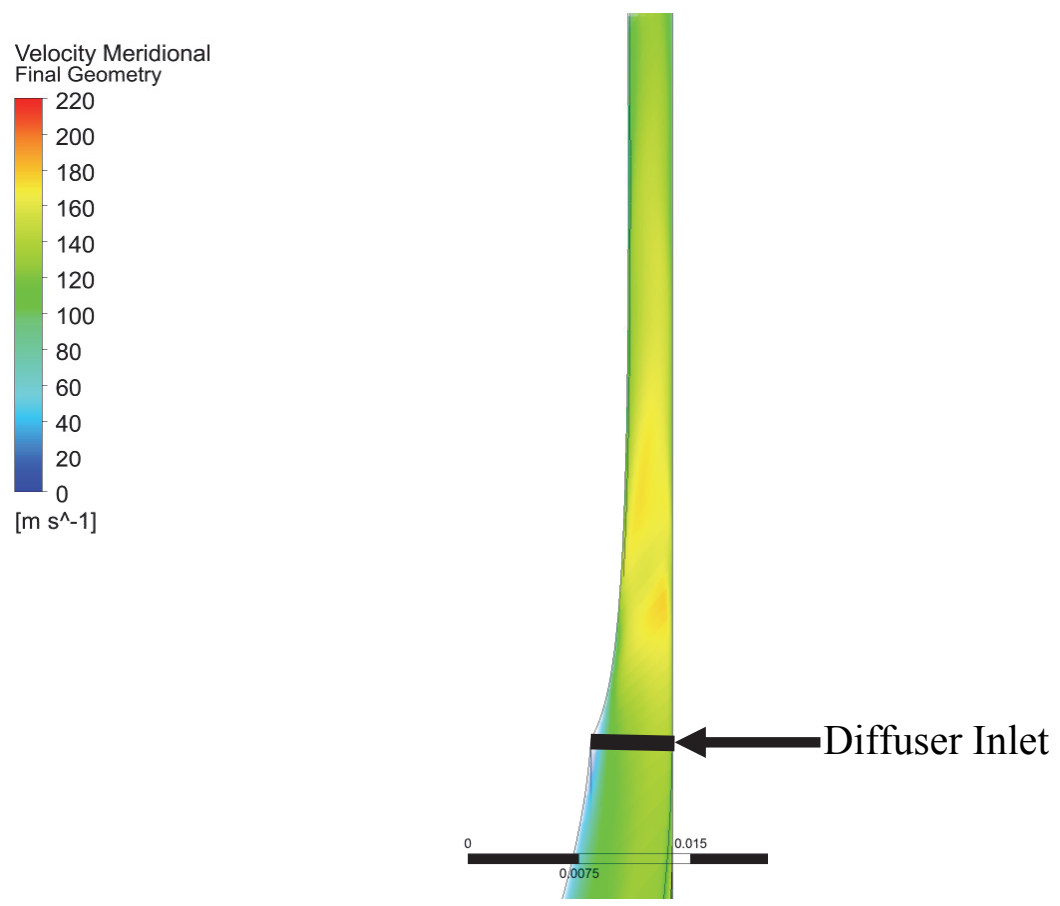

Figure 6-27: Meridional velocity $[\mathrm{m} / \mathrm{s}]$ inside the diffuser corresponding to the final geometry. 


\subsection{Compressor Performance Map: Design Point \#2}

So far, the information presented focused on the design point constant speed line.

The design point constant speed curve is highly important to waste heat recovery systems. Current design criteria indicate that the waste heat recovery system of interest will be attached to a generator which is expected to be connected to the grid. Therefore, the Brayton cycle will operate at a constant speed curve even while off-design. Nonetheless, compressor maps describing the entire operating region of the compressor are required for the design of the control systems. Commonly, characteristic maps are a key tool used during the design of thermodynamic power cycles and the dynamic modelling of such.

The next few figures summarize the compressor performance map corresponding to Design Point \#2. Figure 6-28 illustrate the compressor characteristic map. The map is expressed in the popular turbomachinery form of Pressure Ratio vs Flow Coefficient $\left(\Pi_{\mathrm{m}}\right)$. More information regarding the characteristic maps is presented in Chapter 7. Figure 6-29 illustrates the Efficiency curves versus Flow Coefficient $\left(\Pi_{\mathrm{m}}\right)$. 


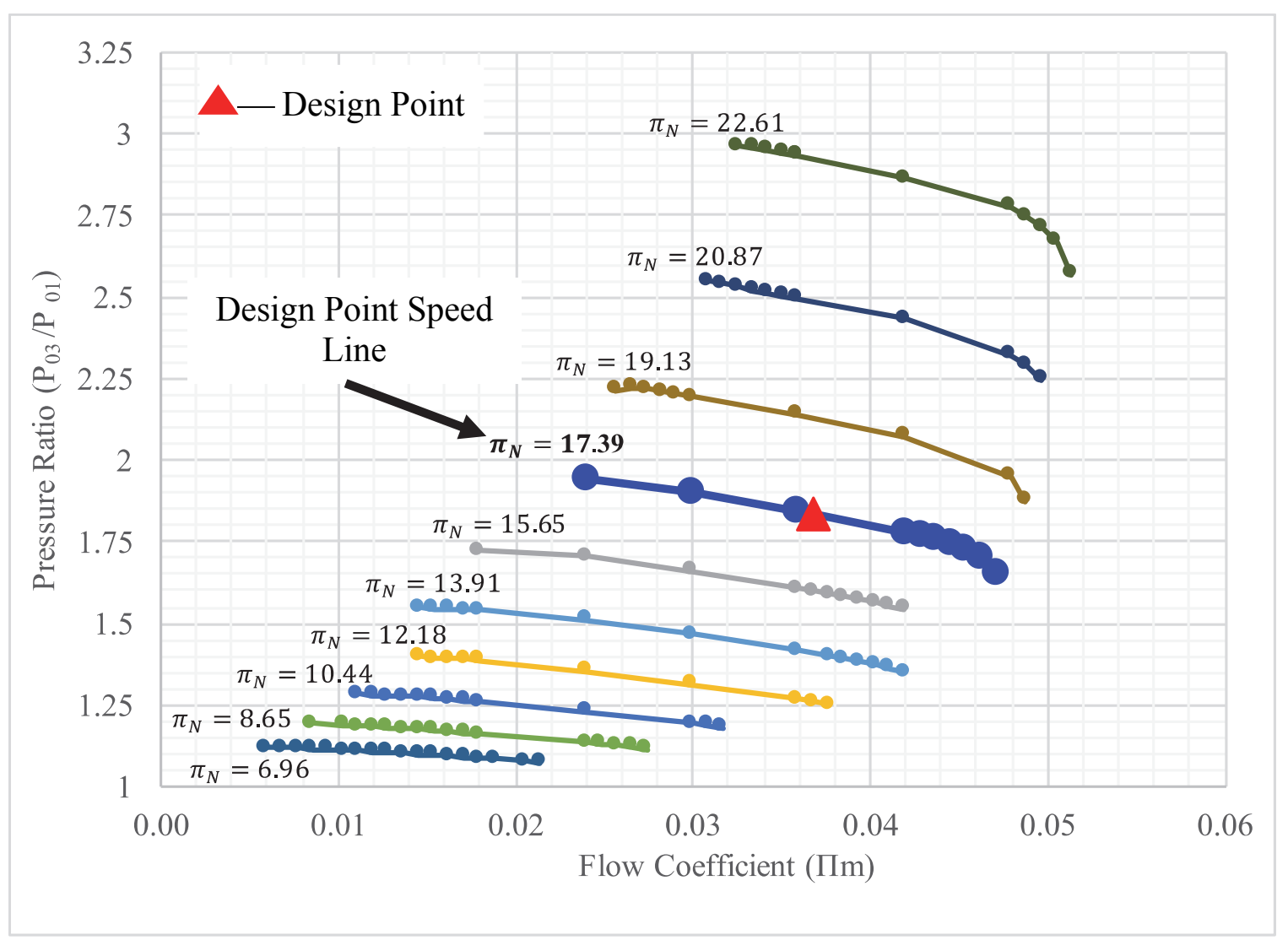

Figure 6-28: Compressor performance map corresponding to Design Point \#2. 


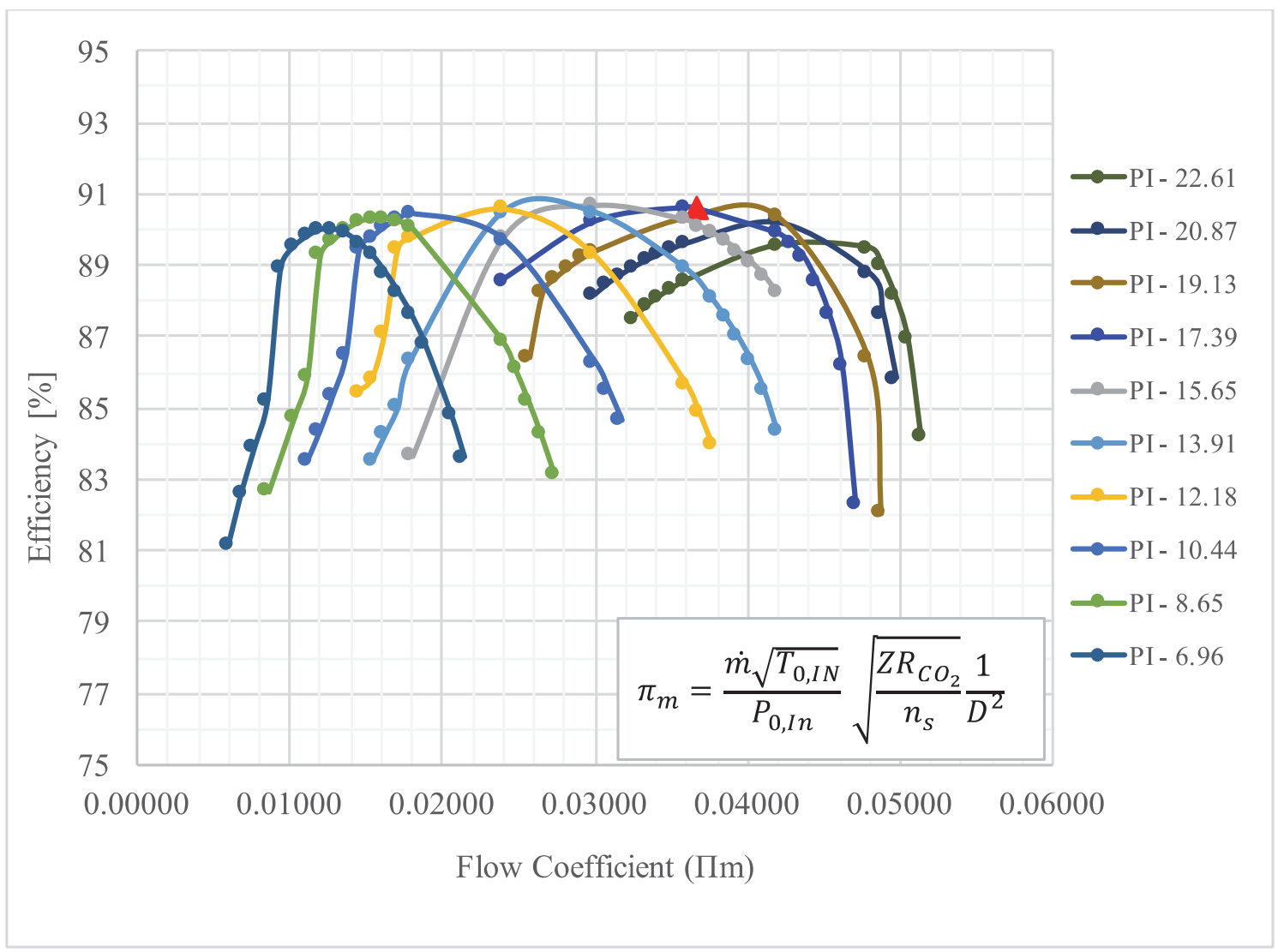

Figure 6-29: Efficiency performance map corresponding to Design Point \#2.

\subsection{Final Geometry}

The results of the $3 \mathrm{D}$ aerodynamic analysis have been presented throughout Chapter 6. Figure 6-30 illustrates the final 3D geometry of the impeller while Figure 6-31 illustrates the final 3D geometry of the "pinch" diffuser. The diffuser was modified to be a pinch diffuser due to evidence of flow separation. From Figure 6-30 (isometric view) one can see that the impeller has an extended inlet. This region was introduced to reduce the perturbations originating from the rotating effects at the inlet of the impeller. Figure 6-32 illustrates the dimensions of the final geometry. 

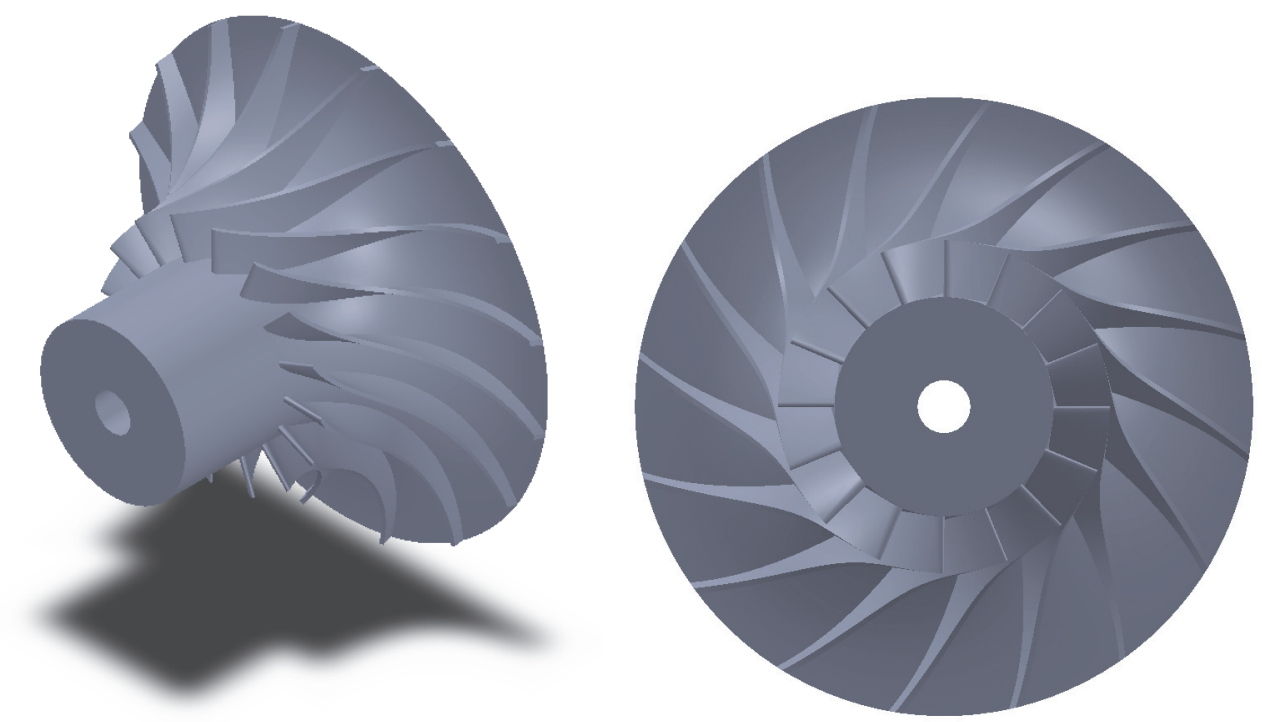

Figure 6-30: 3D geometry of the final preliminary impeller. (Left) Isometric view of the impeller. (Right) front view of impeller.
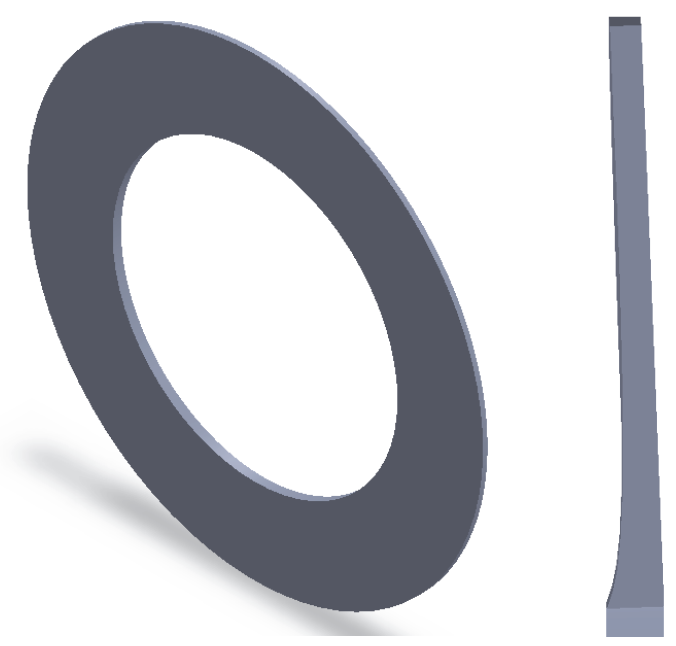

Figure 6-31: 3D geometry of the diffuser. (Left) Isometric view of the diffuser. (Right) Cross-sectional view of the impeller. 


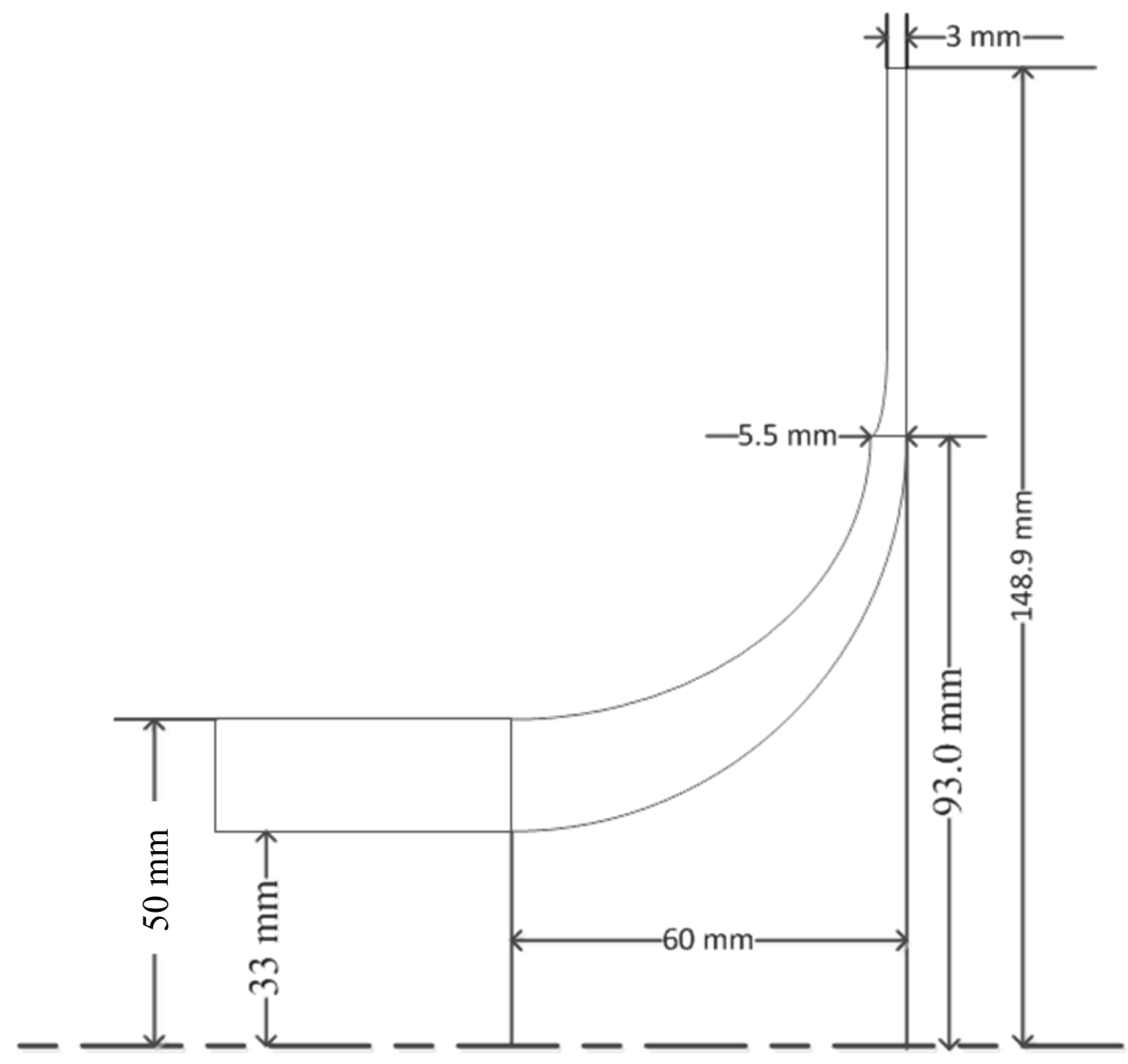

Figure 6-32: Final geometry sizing of the Impeller and diffuser. Note: curvature of impeller and diffuser not to scale.

\subsection{Chapter Summary}

Chapter 6 presented the 3D aerodynamic results corresponding to Design Point \#2. Due to similarity, it is expected that the flow will behave dynamically similarly at Design Point \#1.

- Section 6.2 presented the identified trend between mass flow percent error $\left(\epsilon_{m}\right)$, and the quality of the convergence. When $\epsilon_{m}>0.1 \%$ the convergence and output quality did not satisfy the convergence criteria. 
- Section 6.3 presented a summary of the initial design geometry (V2). It was found that this geometry placed the operating point very close to the "surge" region $\varphi_{\mathrm{e}}=$ 0.19 .

- Section 6.4 presented a summary of the grid sensitivity analysis. The investigation found that the outputs were grid independent. The investigation used a grid of about 740,000 nodes.

- Section 6.5 presented the findings of the aerodynamic study of the final geometry.

○ The final geometry was design to achieve, at the design point, a Flow Coefficient of $\varphi_{\mathrm{e}}=0.35$, a De Haller Number of 0.89 and a Work Coefficient of $\psi=0.69$. The design point had a mass flow surge margin of approximately $\sim 35 \%$. Similarly, the design point achieved a pressure ratio of 1.84 with a CFD efficiency of $\sim 90.5 \%$.

- The diffuser was designed to achieve, at the design point, a pressure recovery of approximately $\sim 42 \%$ at the design point. It was found that the corresponding loss coefficient was about $9 \%$.

- The design point constant speed curve was found to have a percent change between the maximum and minimum pressure ratio of approximately $\sim 13 \%$, similarly the efficiency was found to have a percent change of about $\sim 5 \%$.

- Section 6-6 presented the final geometry characteristic map and efficiency map.

- Section 6-7 illustrated the dimensions of the final geometry and final 3D geometry. 


\section{Chapter 7}

\section{Compressor Performance Maps and Projection Methodology}

\subsection{Introduction}

Chapter 7 focuses on presenting the compressor characteristic maps of Design Point \#1 (Actual Design Point). Section 7.2 presents some of the non-dimensional dependent parameters for real compressible flow. Section 7.2.1 presents the Enthalpy Rise Coefficient and its importance towards expressing compressor maps. Section 7.3 presents the Generalized Map corresponding to the final preliminary geometry. Section 7.4 explains the Projection Methodology. Section 7.5 presents the compressor performance map for Design Point \#1.

\subsection{Dimensional Analysis: Dependent Parameters for Compressible non-ideal Flows}

Chapter 2 described Dimensional Analysis and the importance of nondimensional parameters to turbomachinery. Furthermore, it introduced the effects of real gas on the properties of $\mathrm{CO}_{2}$. It was shown that the compressibility factor $(\mathrm{Z})$ and isentropic exponent $\left(\mathrm{n}_{\mathrm{s}}\right)$ are highly influential parameters for real gas flows. Work by Baltadjiev (2012) provided further details into the effects of the isentropic exponent. Work by Pham et. al (2016) provided further details into the Enthalpy Rise Coefficient. Chapter 2 also introduced the set of independent variables needed to define a real compressible flow as shown by Sjolander (2017). 
In a similar manner to the independent parameter, by using the isentropic exponent $\left(\mathrm{n}_{\mathrm{s}}\right)$ and compressibility factor $(\mathrm{Z})$ one can then express the dependent parameters in a form relevant to the real flow. The incorporation of the two parameters ( $\mathrm{Z}$ and $n_{s}$ ) is commonly achieved through the definition of the speed of sound (Equation 2.22). By this logic, the dependent parameters can be expressed as shown in Table 7-1

Table 7-1: Non-dimensional dependent parameters Enthalpy Rise, Pressure Rise, Power coefficients and expressed for ideal gas behavior and real gas behavior.

\begin{tabular}{|c|c|c|c|}
\hline & Ideal Gas & Real Flow & \\
\hline $\begin{array}{c}\text { Enthalpy Rise } \\
\text { Coefficient }\end{array}$ & $\Pi_{h}=\frac{\Delta h_{0}}{T_{0, I n} R_{C O_{2}} \gamma}$ & $\Pi_{h}=\frac{\Delta h_{0}}{T_{0, I n} R_{C O_{2}} Z n_{s}}$ & Equation 7.1 \\
\hline $\begin{array}{c}\text { Pressure Rise } \\
\text { Coefficient }\end{array}$ & $\Pi_{\mathrm{p}}=\frac{\Delta P_{o}}{\rho_{01} T_{0, I n} R_{C O_{2} \gamma} \gamma}$ & $\Pi_{\mathrm{p}}=\frac{\Delta P_{o}}{\rho_{01} Z R T_{o 1} n_{s}}$ & Equation 7.2 \\
\hline Power Coefficient & $\Pi_{\mathrm{w}}=\frac{\dot{W}}{\rho_{01} T_{0, I n} R_{C O_{2}} \gamma D^{2}}$ & $\Pi_{\mathrm{w}}=\frac{\dot{W}}{\rho_{01} Z R T_{o 1} n_{s} D^{2}}$ & Equation 7.3 \\
\hline
\end{tabular}

Therefore, the parameters summarized in Table 7-1 are all functions of the independent parameters presented in Chapter 2.

The Enthalpy Rise Coefficient $\left(\Pi_{\mathrm{h}}\right)$ has been identified as a key parameter to express the compressor characteristic map.

\subsubsection{Enthalpy Rise Coefficient}

Some work by Pham (2016) has shown evidence that that the Enthalpy Rise Coefficient $\left(\Pi_{h}\right)$ remains relatively constant for dynamically similar operating points. This means that for dynamically similar operating points (Fixed value of $\Pi_{\mathrm{m}}$ and $\Pi_{\mathrm{N}}$ and Re) the Enthalpy Rise Coefficient will remain mostly constant. 
The Enthalpy Rise Coefficient then becomes of great value to turbomachinery designers when it comes to expressing the characteristic map. A constant value of Enthalpy Rise suggests the need to create only one characteristic map. In this thesis, this unique map is referred to as the Generalized Compressor Performance Map $\left(\Pi_{\mathrm{h}} \mathrm{vs} . \Pi_{\mathrm{m}}\right)$, and the Projection Methodology is used to predict the preliminary performance map of Design Point \#1 from the Generalized Compressor Map.

During this investigation, and the design process of the compressor, the Enthalpy Rise Coefficient for different operating conditions of $\mathrm{P}_{\mathrm{o}, \mathrm{In}}, \& \mathrm{~T}_{\mathrm{o}, \mathrm{In}}$ was analyzed. The variation in the inlet operating condition of the compressor provided a range of different values of compressibility factor $(Z)$ and isentropic exponent $\left(n_{s}\right)$. Furthermore, the analysis investigated two other fluids; standard dry air and $\mathrm{CO}_{2}$. The compressor was initially modelled using air to ensure that there were no underlying issues with the simulation prior to modelling with a more complex fluid. These simulations also provided information regarding the troubleshooting and the convergence difficulties. When the Enthalpy Rise Coefficient was investigated, it was found that its value remained constant between all the partially dynamically operating points. Despite modelling with different fluids ( with different values of Gamma $(\gamma)$ ), $\mathrm{Z}$ and $\mathrm{n}_{\mathrm{s}}$ (ideal versus real)) and despite different Reynolds Number, the Enthalpy Rise Coefficient remained relatively constant.

Figure 7-1 illustrates the Enthalpy Rise Coefficient $\left(\Pi_{\mathrm{h}}\right)$ versus Flow Coefficient $\left(\Pi_{\mathrm{m}}\right)$. This figure helps validate the extent to which the Enthalpy Rise Coefficient remains constant. The current investigation provided confidence for expressing the characteristic map in terms of the Enthalpy Rise Coefficient. The validity currently 
extends down to a compressibility factor of $Z=0.65$. Notice that Figure 7-1 does not aim to be considered a complete characteristic map, instead it aims at highlighting the trend in Enthalpy Rise Coefficient $\left(\Pi_{\mathrm{h}}\right)$ as compressibility factor decreases, and different fluids are used.

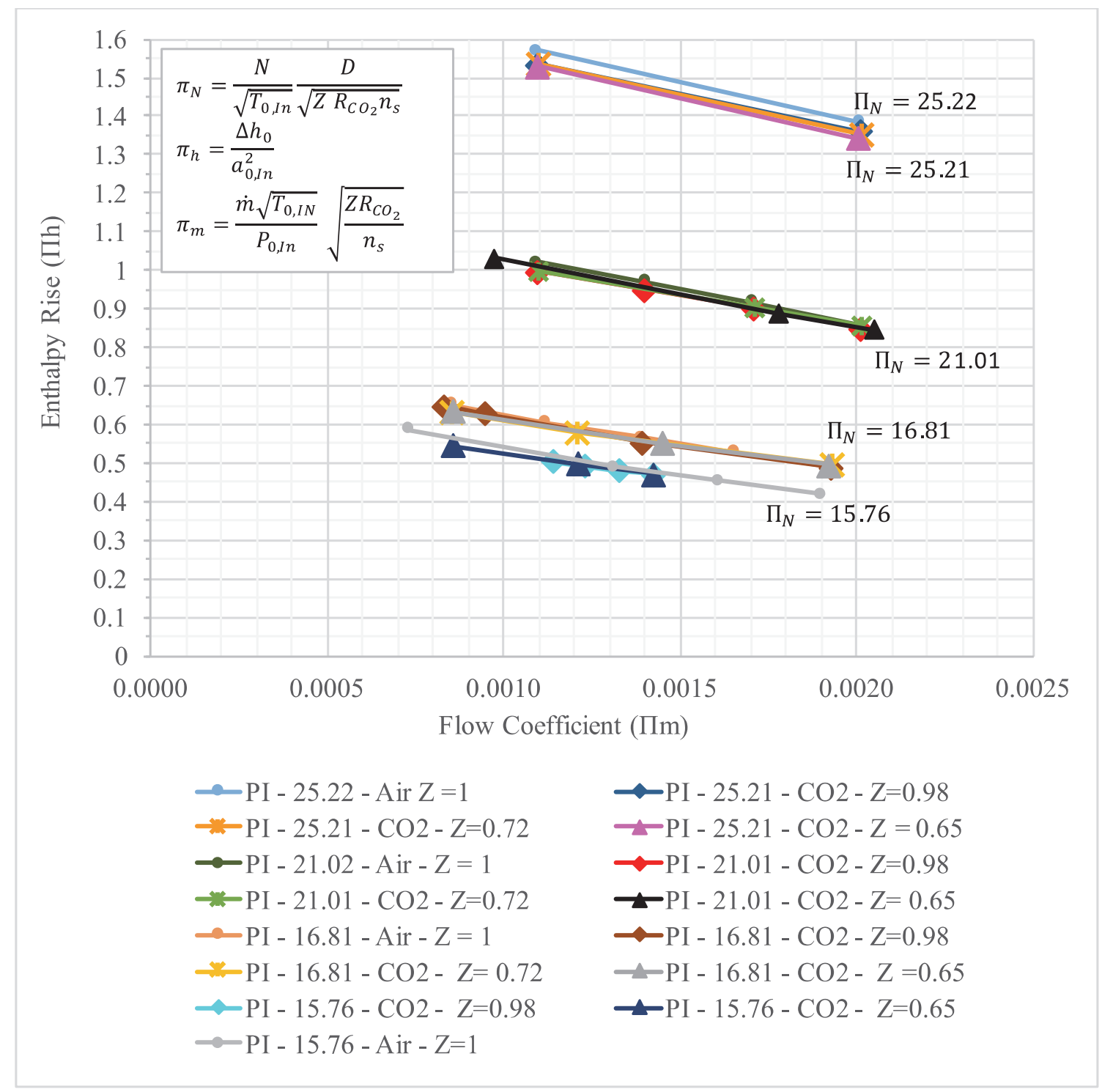

Figure 7-1: Results of the validation study of a constant Enthalpy Rise for different values of Compressibility factor $(Z)$ and different fluids. 
From this investigation and as summarized by Figure 7-1, it is possible to draw several preliminary conclusions regarding the trends in Enthalpy Rise Coefficient.

- The first is that the Enthalpy Rise Coefficient remained relatively constant for partially dynamically similar operating points.

- Secondly, the Enthalpy Rise Coefficient has been validated to remain constant for partially dynamically similar operating points and, most importantly, for different fluids. The figure illustrates values for standard ideal dry air $(Z=1)$, Ideal Supercritical $\mathrm{CO}_{2}(\mathrm{Z}=0.98)$, and $\mathrm{CO}_{2}$ down to a compressibility factor of $\mathrm{Z}=0.72$ and $\mathrm{Z}=0.65$.

- The third important conclusion is that the Enthalpy Rise Coefficient remained constant for different values of the isentropic Exponent $\left(\mathrm{n}_{\mathrm{s}}\right)$.

However, it is important to understand that this investigation only had partial dynamic similarity, and the data for the Enthalpy Rise Coefficient was only obtained for a compressibility factor down to a value of $Z=0.65$. As explained in previous chapters, CFD simulation could not be achieved near the critical point where the $Z$ value is $\sim 0.24$. This data suggest that the Enthalpy Rise Coefficient remains constant despite of the fluid. At the moment, it can be shown that the Enthalpy Rise Coefficient remains constant between air and $\mathrm{CO}_{2}$. This suggests, that a single performance map can express several operating conditions. However, as it will be shown in Section 7.5, further analysis is required to validate the trend in Enthalpy Rise Coefficient down to the critical point. The coefficient may be influenced by the sharp changes in $\mathrm{Z}$ and $\mathrm{n}_{\mathrm{s}}$ at the inlet conditions, therefore further investigation is required. Similarly, the effects of the changing values of $\mathrm{Z}$ and $\mathrm{n}_{\mathrm{s}}$ through the compressions process (inlet to outlet) require further understanding. 
Nonetheless, if, in future investigations, the Enthalpy Rise Coefficient is found to remain constant down to the critical point, then this would suggest that each compressor geometry could be expressed by only one Generalized Compressor Map. Similarly, this would suggest that performance maps corresponding to a given fluid and/or different inlet operating conditions could be derived from the Generalized Map.

\subsection{Generalized Compressor Performance Map}

Recall from Chapter 2, that compressor characteristic maps are commonly expressed in terms of Pressure Ratio versus Flow Coefficient $\left(\Pi_{m}\right)$. Therefore, real compressible flow requires the creation of multiple maps to properly account for the changes in the fluid conditions. However, as shown in Section 7.2.1 the Enthalpy Rise Coefficient is found to be constant for fixed values of $\Pi_{\mathrm{m}}$ and $\Pi_{\mathrm{N}}$, and different values of $\mathrm{Z}$, and $\mathrm{n}_{\mathrm{s}}$ up to a $\mathrm{Z}=0.65$. Consequently, it is possible to express the compressor performance in one single Generalized Characteristic Map, expressed in terms of $\Pi_{h}$

versus $\Pi_{\mathrm{m}}$. Figure 7-2 illustrates the Generalized Compressor Performance Map corresponding to the final geometry. Figure 7-3 illustrates the compressor efficiency map. It is important to remember that the Generalized Map was created from the 3D CFD results corresponding to Design Point \#2. 


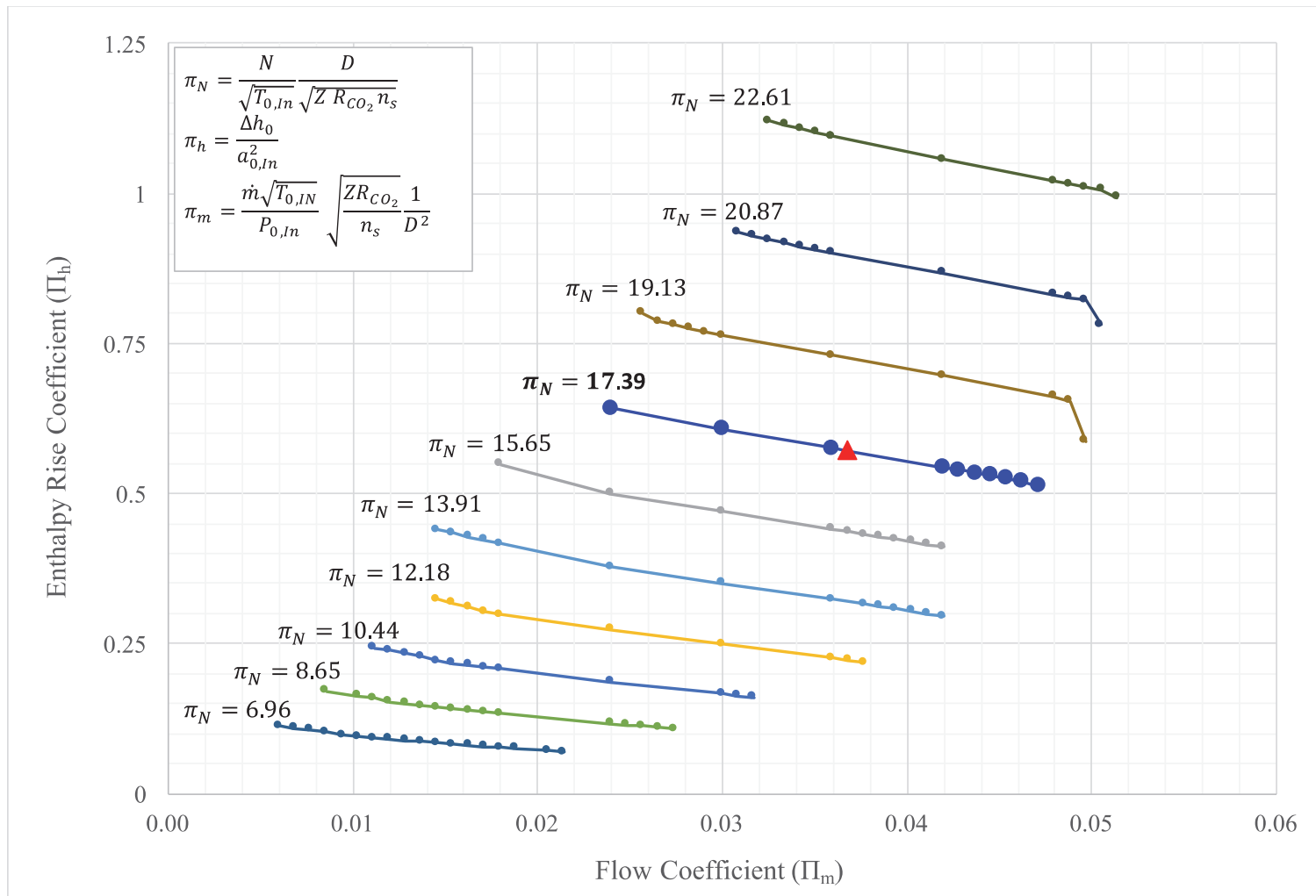

Figure 7-2: Generalized compressor performance map corresponding to the final preliminary geometry. 


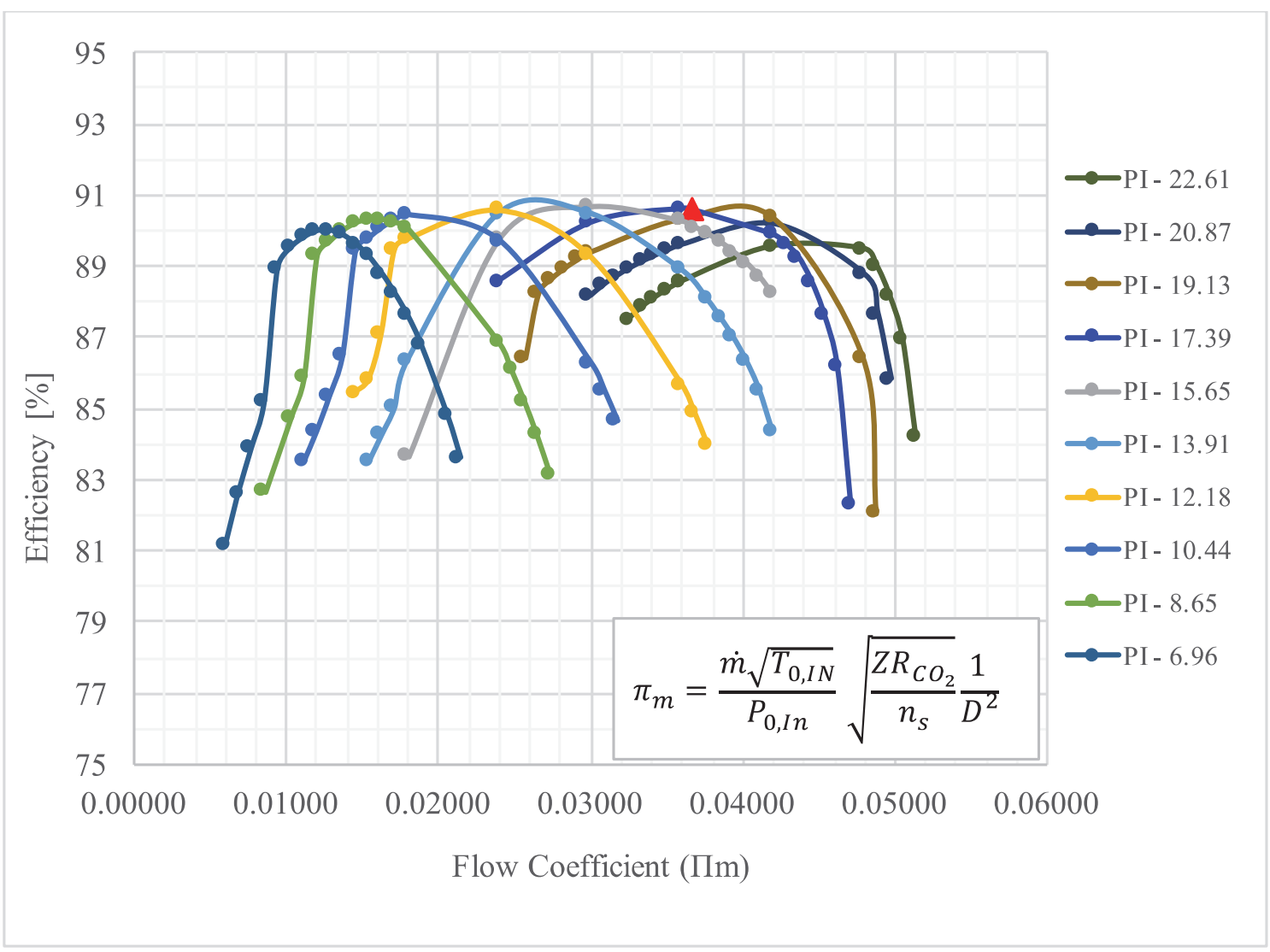

Figure 7-3: Efficiency performance map corresponding to Design Point \#2. It is important to understand that the efficiency corresponds to Design Point \#2. This value is the corrected to represent the estimated efficiency relative to the Reynolds Number of the dynamically similar operating point.

The Generalized Map is a powerful tool for turbomachinery designers in the S$\mathrm{CO}_{2}$ industry. As it was presented in Chapter 2, $\mathrm{S}-\mathrm{CO}_{2}$ experiences sharp changes in properties around the critical point. Therefore, it is understood that several compressor maps need to be created to fully describe the performance of the machine. This can become time consuming during the initial design stages. Hence, using the Generalized Map can be beneficial to the design process. The Generalized Map also provides the opportunity to scale and define characteristic maps between different fluids.

From the Generalized Map, the designer can derive any map for any operating condition in terms of pressure ratio (as long as the built-in assumptions are satisfied). 
Recall that $\Delta \mathrm{P}_{0}$ or $\mathrm{P}_{0, \text { In }} / \mathrm{P}_{0, \text { Out }}$ is a function of $\Delta \mathrm{h}_{0}$ and efficiency. The following Section presents the Projection Methodology. It highlights the steps needed to go from the Generalized Map to any specific operating condition performance map.

\subsection{Projection Methodology}

The Projection Methodology is a term used in this investigation to refer to the set of steps needed to create a compressor map in terms of pressure ratio (PR) and Flow Coefficient $\left(\Pi_{\mathrm{m}}\right)$. As mentioned in the previous section, the Generalized Map allows the designer to derive a compressor characteristic map specific to any combination of total inlet pressure $\left(\mathrm{P}_{0}\right)$ and total inlet temperature $\left(\mathrm{T}_{0}\right)$. Because $\Delta \mathrm{P}_{0}$ or $\mathrm{P}_{0, \mathrm{In}} / \mathrm{P}_{0 \text {, Out }}$ is a function of $\Delta \mathrm{h}_{0}$ and efficiency, then a dynamically similar operating point can be defined in terms of the pressure ratio. Next, a series of steps to define the dynamically similar operating point in terms of pressure ratio is presented.

\section{Projection Methodology}

\section{$\underline{\text { Step } 1}$}

Choose Projection Point

- $\quad$ New operating Condition, $\mathrm{P}_{\mathrm{o} \text {,projection, }} \mathrm{T}_{\mathrm{o} \text {,projection }}$

- Note that the operating condition $\left(\mathrm{P}_{0 \text {,projection, }} \mathrm{T}_{0 \text {,projection }}\right)$ correspond to the inlet total conditions of the compressor.

\section{$\underline{\text { Step } 2}$}

Using a EoS or REFPROP calculate the following inlet properties

$$
\begin{aligned}
& \mathrm{a}_{0, \text { projection inlet }}=\mathrm{f}\left(\mathrm{P}_{0, \text { projection }}, \mathrm{T}_{0, \text { projection }}\right) \\
& \rho_{\text {o,projection inlet }}=\mathrm{f}\left(\mathrm{P}_{0, \text { projection, }}, \mathrm{T}_{0, \text { projection }}\right) \\
& \mathrm{h}_{\text {oprojection inlet }}=\mathrm{f}\left(\mathrm{P}_{0, \text { projection }}, \mathrm{T}_{0, \text { projection }}\right) \\
& \mathrm{s}_{\text {,projection inlet }}=\mathrm{f}\left(\left(\mathrm{P}_{0, \text { projection }}, \mathrm{T}_{0, \text { projection }}\right)\right.
\end{aligned}
$$




$$
\mu_{\text {,projection inlet }}=\mathrm{f}\left(\left(\mathrm{P}_{0, \text { projection, }}, \mathrm{T}_{0, \text { projection }}\right)\right.
$$

\section{$\underline{\text { Step } 3}$}

From Generalized Map choose:

- $\quad$ Speed Coefficient $\left(\Pi_{N}\right)$

- Flow Coefficient $\left(\Pi_{\mathrm{m}}\right)$

- $\quad$ Enthalpy Rise Coefficient $\left(\left(\Pi_{\mathrm{h}}\right)\right.$

- $\quad$ CFD Efficiency $\left(\eta_{\text {Generalize Map }}\right)$

\section{$\underline{\text { Step } 4}$}

Calculate the rotational speed $\left(\mathrm{N}_{\text {projection }}\right)$ and mass flow $\left(\dot{m}_{\text {projection }}\right)$ corresponding to the new Projection point

Using Equation 2.23 and 2.24 solve for $N_{\text {Projected }}$ and $\dot{m}_{\text {Projected }}$

Recall that from Similarity

$$
\begin{aligned}
\Pi_{N_{\text {Projection }}} & =\Pi_{N_{\text {Generalized Map }}} \\
\Pi_{m_{\text {Projection }}} & =\Pi_{m_{\text {Generalized Map }}}
\end{aligned}
$$

\section{$\underline{\text { Step } 5}$}

Calculate the Reynolds Number for the new Projection Point

$$
R e_{\text {Projection }}=\frac{\rho_{\mathrm{oprojection} \mathrm{inlet}_{\text {projection }} * \mathrm{~N}_{\text {Projection }}^{2}}}{\mu_{\text {projection inlet }}}
$$

Calculate the Reynolds Number for the inlet conditions of the Generalize Map

$$
R e_{\text {Generalize Map }}=\frac{\rho_{\mathrm{o}_{\text {Generalize Map }}} * \mathrm{~N}_{\text {Generalize Map }} * D_{\text {Generalize Map }}^{2}}{\mu_{\text {Generalize Map }}}
$$

\section{$\underline{\text { Step } 6}$}

Apply Reynolds Number correction to CFD efficiency

$$
\eta_{\text {Correction }}=1-\left(1-\eta_{\text {Generalize Map }}\right) *\left(\frac{R e_{\text {Generalize Map }}}{R e_{\text {projection }}}\right)^{n}
$$

where:

$$
\begin{aligned}
& n=0.1 \text { for centrifugal compressor } \\
& n=0.2 \text { for axial compressors }
\end{aligned}
$$




\section{$\underline{\text { Step } 7}$}

Solve for the actual total enthalpy change $\left(\Delta \mathrm{h}_{0, \text { Projection }}\right)$ at new Projection Point

$$
\Delta \mathrm{h}_{0, \text { Projection }}=\left(\frac{\Delta \mathrm{h}_{0,}}{a_{0, I n}^{2}}\right)_{\text {Generalize Map }} * a_{0, \text { projection inlet }}^{2} \quad \text { Equation (7.5) }
$$

\section{$\underline{\text { Step } 8}$}

Determine the projected isentropic total enthalpy $\left(\Delta \mathrm{h}_{0 \text {, projected Outlet }}\right)$ at the outlet of the component.

$$
\mathrm{h}_{0, \text { Projection Outlet }}^{\prime}=h_{0, \text { projection Inlet }}+\Delta \mathrm{h}_{0, \text { Projection }} \quad \text { Equation (7.6) }
$$

\section{Step 9}

Use REFPROP to determine outlet total pressure $\left(\mathrm{P}_{0 \text {, projected Outlet }}\right)$ and corresponding pressure ratio

$\mathrm{P}_{0, \text { projected Outlet }}=\mathrm{f}\left(\mathrm{h}_{0, \text { projected Outlet, }}, \mathrm{S}_{\text {projection inlet })}\right.$

Pressure Ratio $=\mathrm{P}_{0 \text {, projected Outlet }} / \mathrm{P}_{0 \text {, projected Inlet }}$

\section{Step 10}

Repeat Steps 3 to 9 until all desired operating points in the Generalize Map have been converted to Mass Flow Coefficient $\left(\Pi_{\mathrm{m}}\right)$ versus Pressure Ratio for the new Projection Point.

Notice that Step 6 refers to a Reynolds Number correction on the efficiency. Recall from Chapter 2, that for two operating points to be fully dynamically and geometrically similar all the independent variables must match. As shown in Chapter 4, the current investigation only matched the Flow and Speed Coefficient between Design Point \#1 and Design Point \#2 making it partially dynamically similar. As explained earlier, the Reynolds Number was not matched due to the fixed geometry diameter. Nonetheless, it is known that the primary effects of Reynolds Number are reflected in the efficiency. Similarly, as shown in Sjolander (2017), the effects for Reynolds Number on efficiency can be corrected by applying Equation 2.26. It is important to understand that this correction will only affect the efficiency when there is a large difference in Reynolds Number between the two operating conditions.

The set of steps previously presented can then be repeated for each operating point on the Generalized map. This new set of values then provides enough information 
to represent the characteristic map in the traditional way. The following section illustrates the compressor map corresponding to Design Point \#1.

\subsection{Compressor Performance Map: Design Point \#1}

Recall that the geometry boundary conditions and 3D aerodynamic investigation used Design Point \#2 as the reference point. Operating conditions of Design Point \#2 were chosen to ensure ideal gas behavior and facilitate the CFD analysis. Similarly, partial dynamic similarity was maintained between the two operating points. The 3D aerodynamic results obtained through CFD allowed the creation of the Generalized Map. Following the Projection Methodology, it was possible to express the characteristic performance map corresponding to Design Point $\# 1$ as shown in Figure 7-4. In a similar

manner, by applying the Reynolds Number correction one can express the efficiency map corresponding to Design Point \#1 as shown in Figure 7-5. 


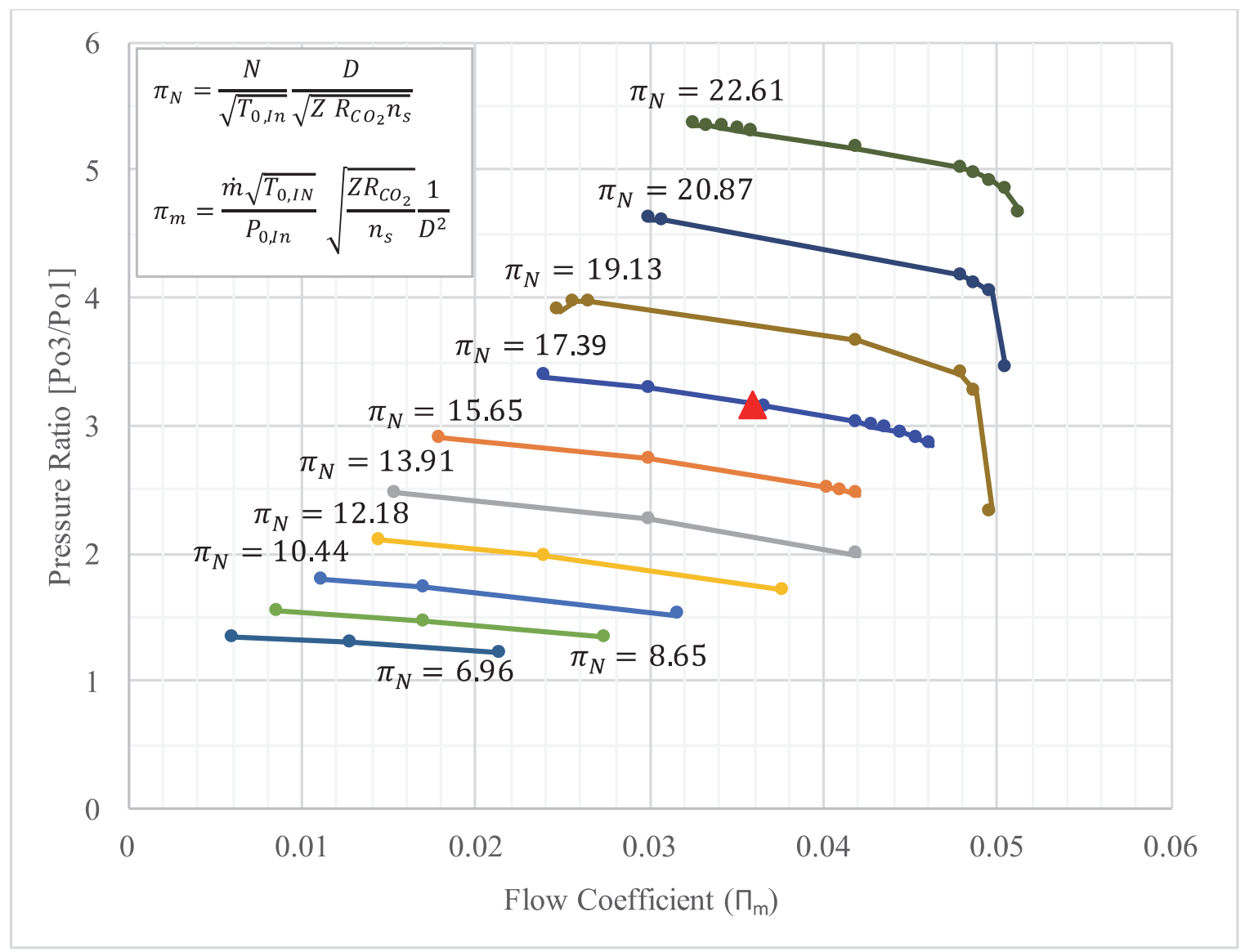

Figure 7-4: Compressor performance map corresponding to Design Point \#1. 


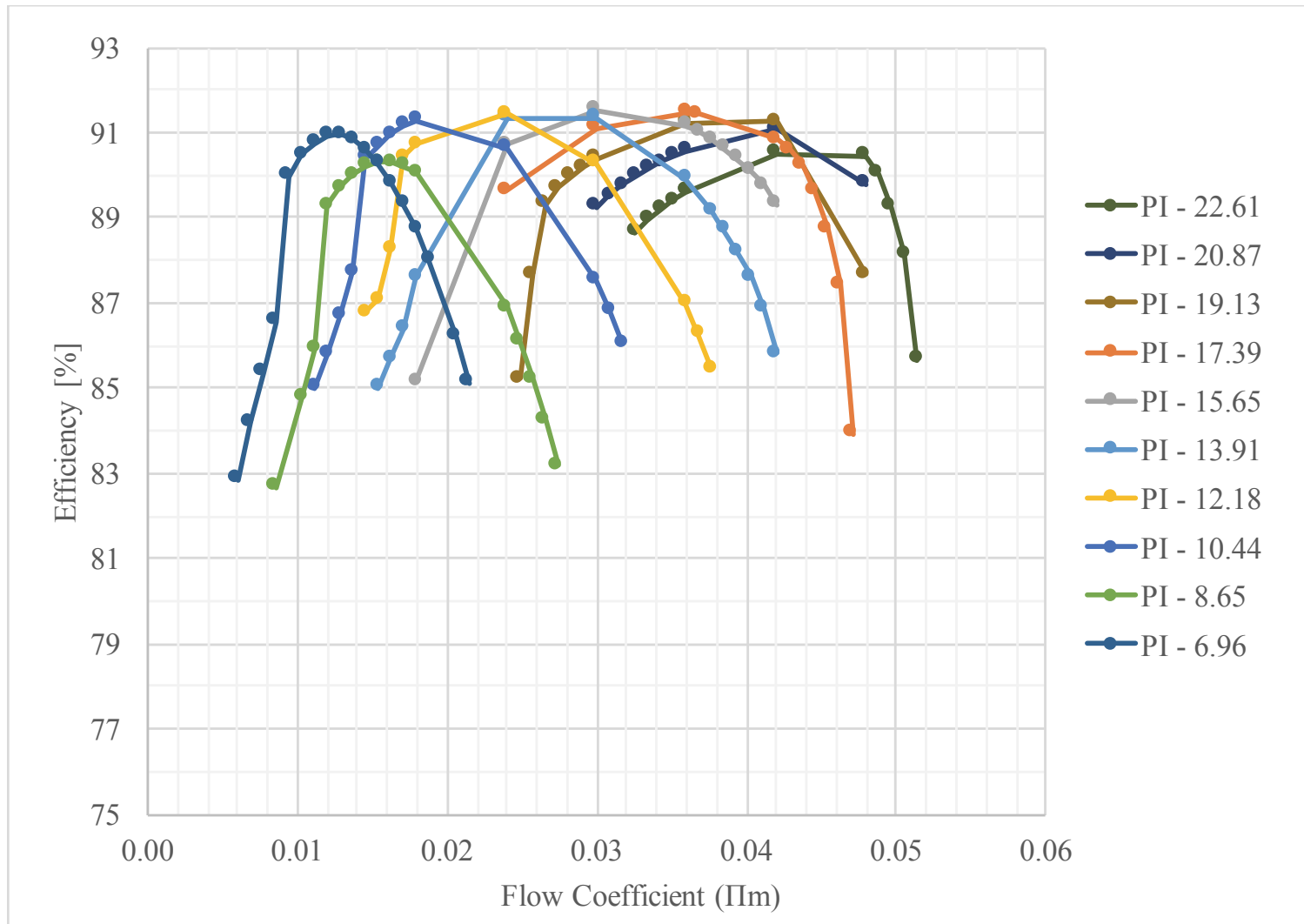

Figure 7-5: Compressor efficiency map corresponding to Design Point \#1.

Notice that the compressor map corresponding to Design Point \# 1 (Figure 7-4) is an approximation. Recall that the use of the isentropic exponent $\left(\mathrm{n}_{\mathrm{s}}\right)$ assumes a constant value of the isentropic exponent. Therefore, the use of the isentropic exponent in the nondimensional parameters is limited to the same assumption. This implies that as the isentropic exponent $\left(n_{s}\right)$ deviates from a constant value, an error could be introduced to the independent parameters that use $n_{s}$. At the moment, the degree of error related to using isentropic values that are not constant is not well understood, and further investigation is necessary.

Figure 7-6 illustrates the changes in the isentropic exponent versus temperature for constant isobars. For an isobar $(\mathrm{P}=7.39$ - blue line) near the critical pressure the 
isentropic exponent remained constant until the critical temperature $\left(T_{c}=304.13 \mathrm{~K}\right)$. It is possible to observe that for each isobar, the temperature at which the isentropic exponent started to exhibit change increased with pressure and temperature. As mentioned in Chapter 2, the changes in some properties of $\mathrm{S}-\mathrm{CO}_{2}$ follow the pseudocritical line, and therefore the pseudocritical region. Figure 7-6, (Black dashed line) illustrates the isentropic exponent values corresponding to the pseudocritical line. However, it is not possible to see a relation between the temperature at which the isentropic exponent starts to change and the pseudocritical temperature.

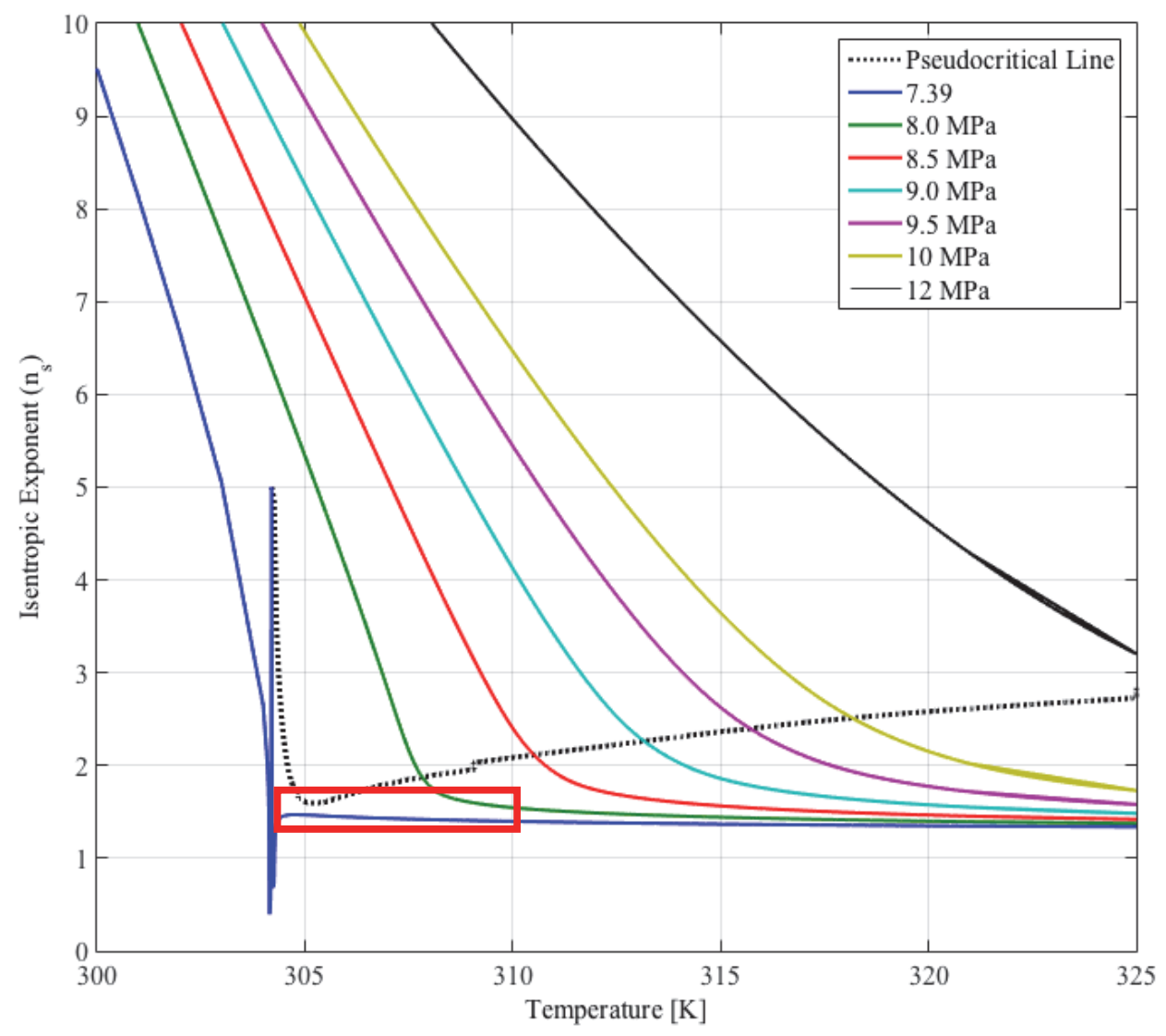

Figure 7-6: Value of isentropic exponent as a function of temperature for different isobars. The isentropic value corresponding to the pseudocritical line is illustrated by the black dash line. 
Figure 7-6 suggest that performance maps that are projected to pressures above the critical pressure and temperatures between 304.13 and $>325$ can potentially have a higher error due to the sharp changes in $n_{s}$ value. However, as previously mentioned, the moment the degree of error incurred by not using constant isentropic values is not well known and requires further investigation.

When projecting maps to a region near the critical point (as the region highlighted by the red rectangle), in the case of most compressors designed for $\mathrm{S}_{-} \mathrm{CO}_{2}$ Brayton cycles, there is a possibility that the maps will incorporate low discrepancies between projected map and actual CFD simulations. This hypothesis could remain true as the isentropic exponent shows small changes between ideal gas region and real gas region. However, during the compression process, the isentropic exponent experience large changes between the inlet and the outlet and further investigations are required to understand the effects of this change.

\subsection{Benefits of Generalize Map and Projection Methodology}

As mentioned in Section 7.2.1 expressing the compressor performance map in terms of the Enthalpy Rise Coefficient (as the Generalize Map) can be a powerful tool. From the Generalized Map, by applying the Projection Methodology one can derive preliminary performance map at different compressor inlet conditions. Table 7-2 summarizes three different compressor inlet operating conditions. These, conditions were used in the projection methodology to project three different performance maps (Figure 7-7 to Figure 7-9). 
Table 7-2: Summary of the three different Projection Points used to create Figure 7-7.

\begin{tabular}{ccccc}
$\begin{array}{c}\text { Projection } \\
\text { Temperature } \\
\left(\mathbf{T}_{01}\right)[\mathrm{K}]\end{array}$ & $\begin{array}{c}\text { Projection } \\
\text { Pressure }\left(\mathbf{P}_{01}\right) \\
{[\mathrm{MPa}]}\end{array}$ & $\begin{array}{c}\text { Density }\left(\rho_{0,1}\right) \\
{\left[\mathrm{kg} / \mathrm{m}^{3}\right]}\end{array}$ & $\begin{array}{c}\text { Isentropic } \\
\text { Exponent }\left(\mathrm{n}_{\mathbf{s}}\right)\end{array}$ & $\begin{array}{c}\text { Reynolds } \\
\text { Number }(\mathbf{R e})\end{array}$ \\
\hline & 7.6 & 288 & 1.46 & $8.106 \times 10^{9}$ \\
$\mathbf{3 0 8 . 1 5}$ & 8.40 & 595 & 3.57 & $9.787 \times 10^{9}$ \\
& 9.00 & 662 & 5.59 & $1.149 \times 10^{10}$ \\
\hline
\end{tabular}

The following set of figures illustrate what the performance map of the compressor could look like at each of the inlet conditions $\left(\mathrm{P}_{\mathrm{o}, 1}\right.$ and $\left.\mathrm{T}_{\mathrm{o}, 1}\right)$. At the moment, these are solely approximations of the compressor performance. As previously mentioned, further validation is needed regarding the Enthalpy Rise Coefficient. Similarly, more understanding is required regarding the effects of changing the values of isentropic Exponent and compressibility factor. Therefore, the validity of the Projection Methodology is subjected to the current validity of the Enthalpy Rise Coefficient down to a compressibility factor of $Z=0.65$.

Nonetheless, these maps suggest that the performance of the machine varied with inlet conditions as one would expected. These changes can be seen through the speed lines. The design point speed line, and corresponding design point achieved a different pressure ratio for each of the inlet conditions. The extent to which the Pressure Ratio is affected needs further investigation.

If the Enthalpy Rise Coefficient is validated down to the critical point, and it is found that through the projection methodology the pressure ratio is accurately predicted then this will fully support the validity of the Generalized Map. This would indicate wit certainty that the performance of a geometry could be expressed by a single Generalized Map, and the corresponding performance map (for different inlet conditions or different fluids) could be predicted from the Generalized Map. However, as mentioned before 
further understanding of the effects of changing $\mathrm{Z}$ and $\mathrm{n}_{\mathrm{s}}$ are required. Similarly, the Enthalpy Rise Coefficient would need to be validate down to the critical point. To further validate the Projection Methodology it would also be necessary to validate the Reynolds Number correction formula. As explained, the Reynolds Number mainly affects the efficiency of the machine, which is currently derived from CFD. Because the Re could change between the Generalize Map and the projection points, it is necessary to investigate if the correction equation accurately predicts the changes in efficiency due to Reynolds Number.

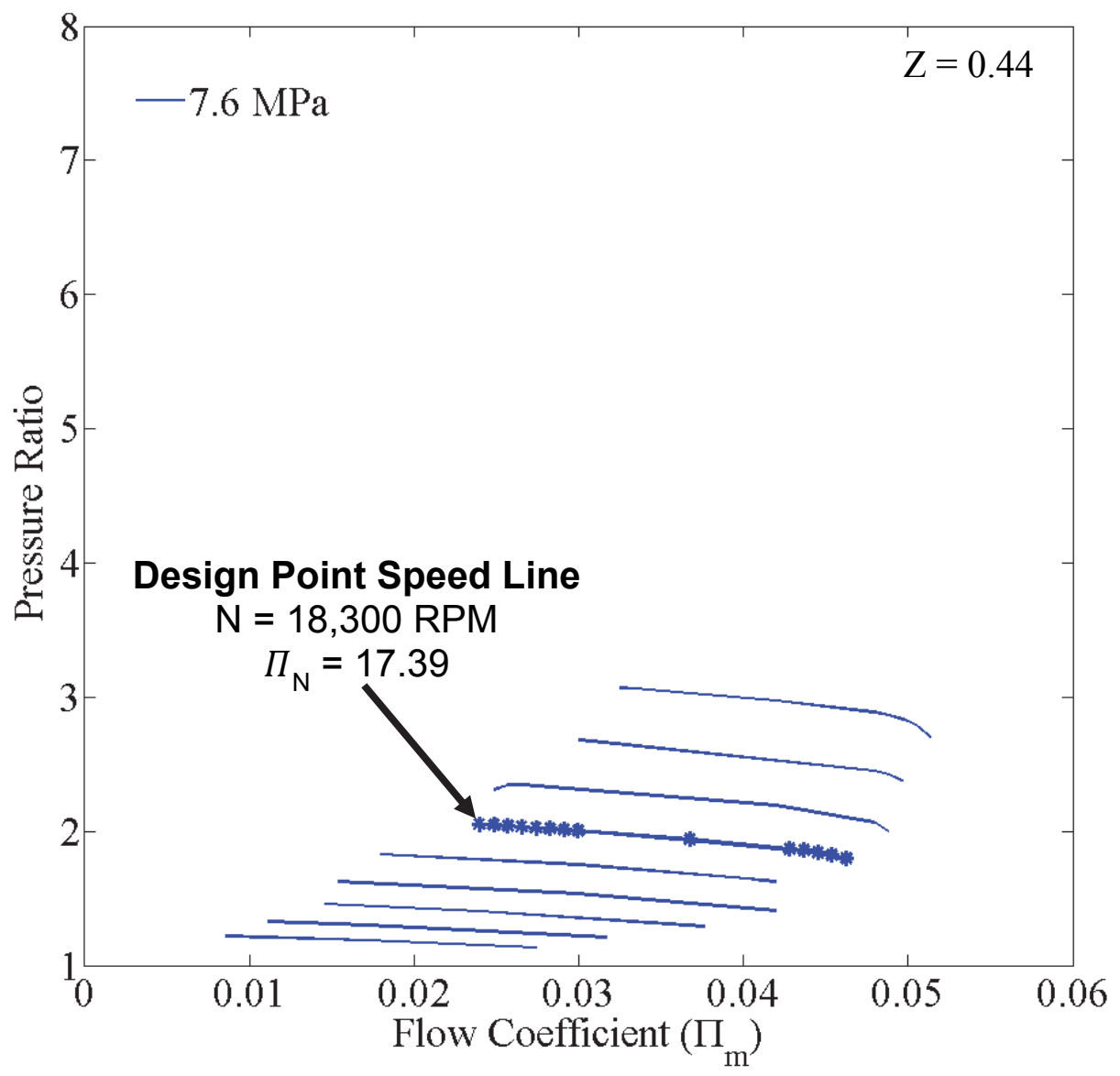

Figure 7-7: Preliminary Performance Map for compressor inlet conditions of $\mathrm{P}_{\mathrm{o}, 1}=7.6 \mathrm{MPa}$ and $\mathrm{T}_{\mathrm{o}, 1}=$ $304.15 \mathrm{~K}$. 


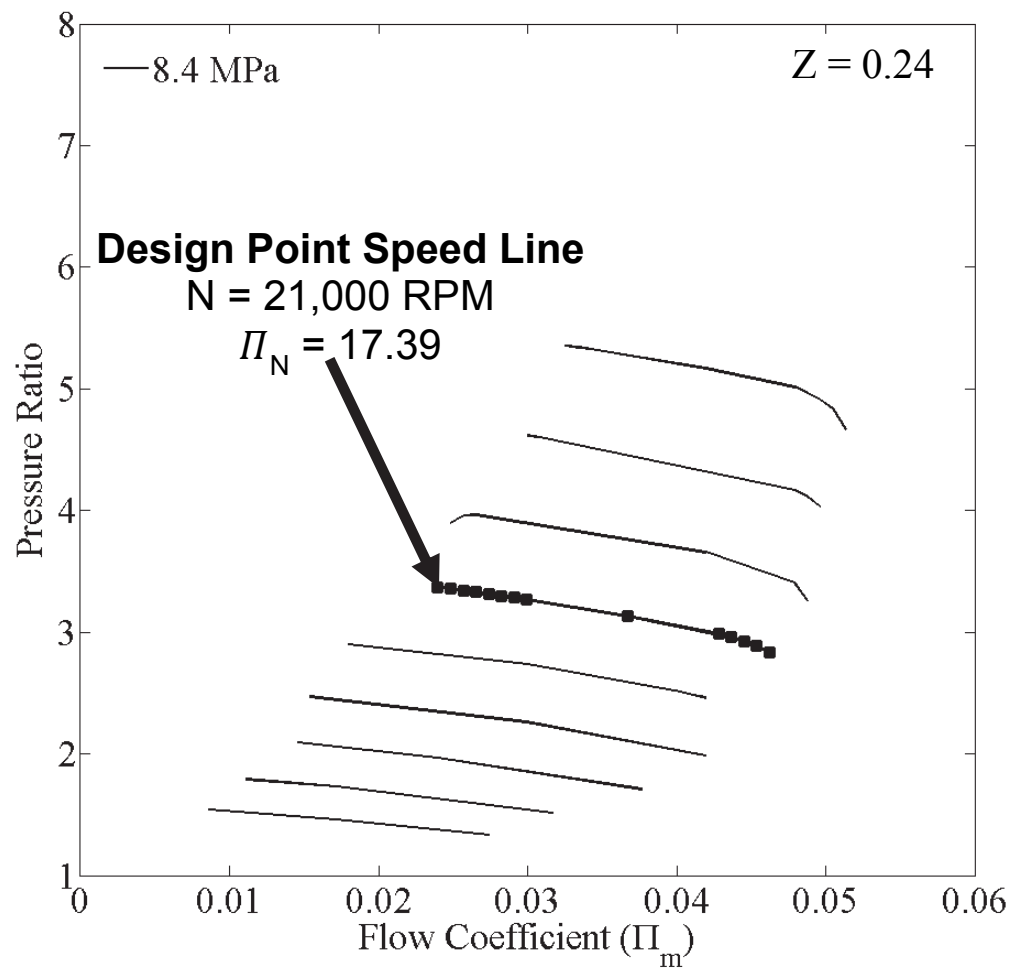

Figure 7-8: Preliminary Performance Map for compressor inlet conditions of Po, $1=8.4 \mathrm{MPa}$ and To, $1=$ $304.15 \mathrm{~K}$.

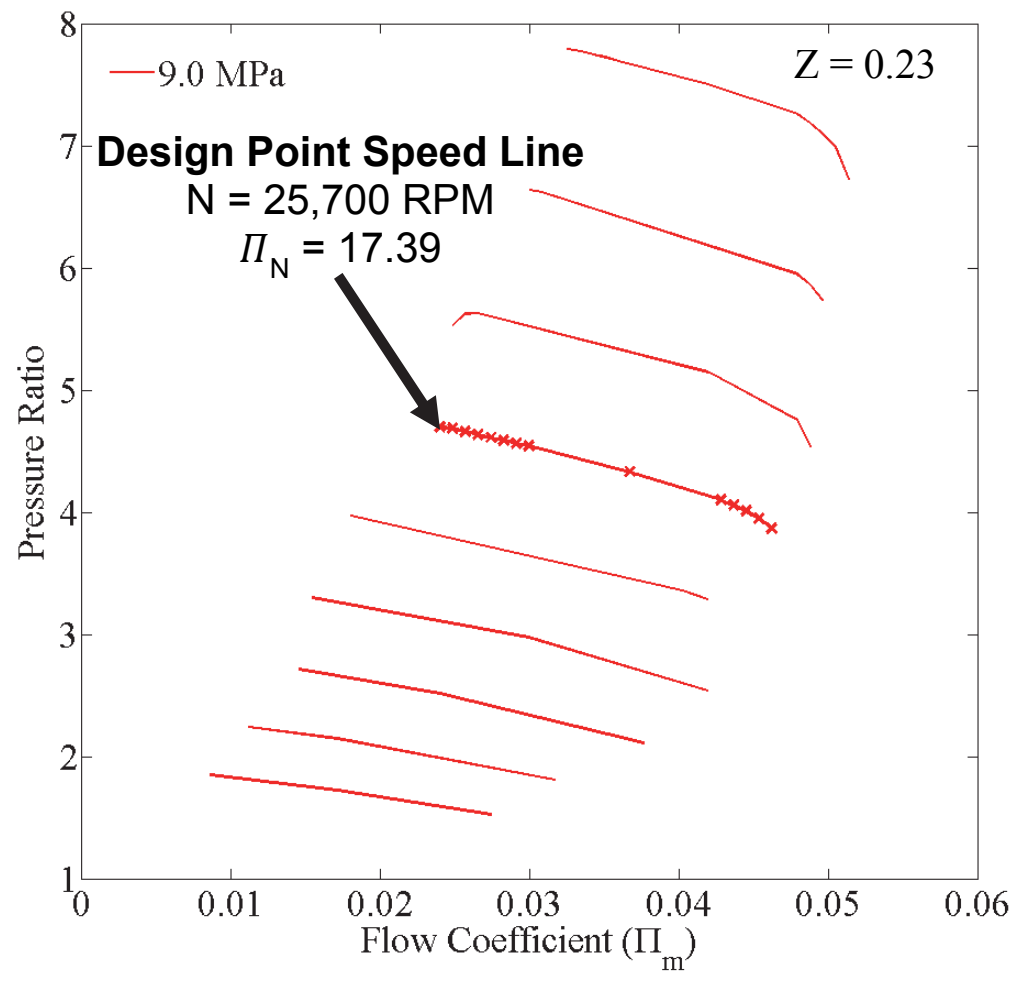

Figure 7-9: Preliminary Performance Map for compressor inlet conditions of Po, $1=9.0 \mathrm{MPa}$ and To, $1=$ $304.15 \mathrm{~K}$. 
The investigation also tried to identify a trend between the projected performance maps and the effects of the isentropic exponent. However, there is not enough information to directly relate the isentropic exponent to the changes in performance seen at each of the projected points.

The compressor map corresponding to a pressure near the critical point $\left(\mathrm{P}_{01}=7.6\right.$ $\mathrm{MPa}$ - blue line) experienced the least amount of pressure rise. The map corresponding to a pressure of $9 \mathrm{MPa}$ experienced the largest pressure rise. The changes in pressure rise could be associated to the fluid density. Recall Chapter 4 and the discussion regarding density. It was shown that for an isotherm (for example $\mathrm{T}=308.15 \mathrm{~K}$ ) as pressure increased so did the value of density. Larger values of density, less energy will be required to achieve a given pressure rise. Therefore, the changes in pressure ratio shown in Figure 7-7 to Figure 7-9 could be related to the different values of density as shown in Table 7-2. However, further modelling is required to fully validate these projections as previously mentioned.

\subsection{Chapter Summary}

- Section 7.2 presented the dependent non-dimensional parameters corresponding to real compressible flows.

- Section 7.2.1 presented the Enthalpy Rise Coefficient $\left(\Pi_{\mathrm{n}}\right)$ and how it remained constant for fixed values of Speed $\left(\Pi_{\mathrm{N}}\right)$ and Flow Coefficient $\left(\Pi_{\mathrm{m}}\right)$. 
- Section 7.3 presented the Generalized compressor performance map, which is expressed in terms of the Enthalpy Rise Coefficient $\left(\Pi_{h}\right)$ as a function of Speed $\left(\Pi_{N}\right)$ and Flow Coefficient $\left(\Pi_{\mathrm{m}}\right)$.

- Section 7.4 explained the Projection Methodology and the necessary steps to express any operating point in the Generalized Map in terms of pressure ratio.

- Section 7.5 Presented the compressor characteristic map (in terms of pressure ratio) and efficiency map corresponding to Design Point \#1. This section also highlighted the changes seen in the isentropic exponent $\left(\mathrm{n}_{\mathrm{s}}\right)$.

- Section 7.6 presented an example of the benefits of using the Generalized Map and Projection Methodology. However, as expressed in previous sections further investigation is required to fully validate the accuracy of the Projection Methodology near the critical point. 


\section{Chapter 8}

\section{Conclusion and Recommendations}

\subsection{Conclusions}

The investigation focused on developing the preliminary geometry of a centrifugal compressor for a $10 \mathrm{MW}_{\mathrm{e}}$ waste heat recovery power plant. The preliminary design of the geometry was achieved by applying common centrifugal compressor design guidelines. The design point of the compressor is located near the critical point of $\mathrm{CO}_{2}$. Near that region sharp changes in properties are observed. The non-ideal gas behavior of $\mathrm{CO}_{2}$, as well as the use of Real Gas Property files caused challenges in the convergence of the CFD. Due to the time constraints of the project and the need to determine the performance map and preliminary dimensions of the geometry non-dimensional parameters were used to model a partially dynamically similar operating point.

The non-dimensional parameters were used to determine a geometrically and partially dynamically similar operating point. This operating point remained within the supercritical regime of $\mathrm{CO}_{2}$, however it was chosen to be within the region where $\mathrm{CO}_{2}$ behaves as an ideal gas. Operating in the ideal gas region allowed for the proper convergence of the CFD. Through similarity one could then approximate the performance of the machine at the desired design point. 
The investigation used non-dimensional parameters which incorporate the compressibility factor $(\mathrm{Z})$ and isentropic exponent $\left(\mathrm{n}_{\mathrm{s}}\right)$. The use of these parameters further enhances the description of non-ideal gas flows as shown by Baltadijiev (2012). However, due to the nature of the isentropic exponent, further analysis is required to fully understand its significance and effects on the independent non-dimensional parameters.

The investigation used the dependent non-dimensional parameter Enthalpy Rise Coefficient $\left(\Pi_{h}\right)$, which has been previously presented by Pham et al, (2016). This investigation determined a region where this dependent parameter remains constant for a given value of Speed and Flow Coefficient. The investigation validated the Enthalpy Rise Coefficient down to a compressibility factor of $Z=0.65$. It also compared the values of Enthalpy Rise Coefficient between air and ideal $\mathrm{CO}_{2}$. It was found that its value for a given Speed and Flow Coefficient remained constant between the different fluids. Further investigation is required to fully validate the Enthalpy Rise Coefficient down to a low compressibility factor near the critical point.

Then Enthalpy Rise Coefficient can be highly beneficial to turbomachinery. It was found that this parameter can be used to expressed the compressor characteristic map as a single unique map, referred to as the Generalized Compressor Map. Through the application of scaling, and the relation of enthalpy rise $\left(\Delta \mathrm{h}_{0}\right)$ and efficiency $(\eta)$ to pressure rise $\left(\Delta \mathrm{P}_{0}\right)$, it is possible to derive the characteristic map corresponding to a specific operating condition $\left(\mathrm{P}_{0}, \mathrm{~T}_{0}\right)$. These set of maps are referred to as the projected characteristic compressor maps in this investigation. However, it is important to understand that the accuracy of the projected characteristic compressor maps near the 
critical point are currently limited to the validity of the Enthalpy Rise Coefficient as well as the $\mathrm{Z}$ and $\mathrm{n}_{\mathrm{s}}$ near the critical point.

In conclusion, the investigation provided the preliminary design of a centrifugal compressor. A powerful method of describing characteristic maps, under one unique map was presented further investigations are suggested to fully validate the Generalize Map. A Generalized Compressor Map was developed for the geometry of interest and tentative projected characteristic maps corresponding to the design point were derived.

\subsection{Recommendations}

The investigation focused only on the preliminary geometry design of the machine. It is recommended that stress analysis be performed and the geometry be updated accordingly. Depending on the findings of the stress analysis, the blade thickness of the vanes could potentially be reduced.

It is also recommended that a new $3 \mathrm{D}$ aerodynamic analysis be completed using surface roughness. The modelling of the actual material and surface roughness will help predict more accurate efficiencies.

Further investigation is necessary to understand the influence of the compressible real gas effects on the independent non-dimensional parameters. Similarly, this requires further understanding of the effects of the isentropic exponent. The use of this parameter is built upon the assumption of a constant value. However as it was explained, the isentropic exponent rapidly changes near the critical point. Better understanding the effects of the isentropic exponent $\left(n_{s}\right)$ and compressibility factor $(Z)$ on the independent non-dimensional parameter will provide higher certainty in the scaling of dynamically 
similar operating points. Better understanding will also provide higher certainty in the use of the non-dimensional parameters described in Sjolander (2017). Similarly, further information can increase certainty regarding the Projection Methodology for operating points near the critical point.

It is recommended that a validation of the Enthalpy Rise Coefficient be performed for compressibility factor $(\mathrm{Z})$ of 0.65 and below. An investigation along the mentioned compressibility factor will provide further information to validate the Enthalpy Rise Coefficient and in effect validate the accuracy of the Generalized compressor map near the critical region. Recent work by GE (Moraga 2017, and Saxena 2017) presented at Turbo Expo 2017 showcase an approach to model near the critical region. This approach could potentially be employed in CFX as an option to model near the critical region. 


\section{References}

ANSYS Inc., (2016). ANSYS TurboSystem User's Guide. Release 17.0. Canonsburg, PA, USA.

ANSYS Inc., (2016). ANSYS TurboGrid User's Guide. Release 17.0. Canonsburg, PA, USA.

ANSYS Inc., (2016). "ANSYS Workbench Help" TurboSystem - Chapter 9. BladeGen, Release 17.2. Canonsburg, PA, USA.

ANSYS Inc., (2016). ANSYS TurboGrid Introduction. Release 17.1. Canonsburg, PA, USA.

ANSYS Inc., (2016). ANSYS CFX-Pre User's Guide. Release 17.1. Canonsburg, PA, USA.

ANSYS Inc., (2016). ANSYS CFD-Post User's Guide. Release 17.2. Canonsburg, PA, USA.

Aungier R.H., (1993). Aerodynamic Design and Analysis of Vaneless Diffusers and Return Channels. Proceedings of the ASME Turbo Expo, Paper No. 93-GT-101, Cincinnati, USA.

Aungier R.H., (1995). Centrifugal Compressors Stage Preliminary Aerodynamic Design and Component Sizing. Proceedings of the ASME Turbo Expo, Paper No. 95-GT-78, Houston, USA.

Aungier R.H., (2000). Centrifugal Compressors: A Strategy for Aerodynamic Design and Analysis. ASME Press, New York, USA.

Balje. O.E, (1981), Turbomachines: A Guide to Design, Selection and Theory, Wiley and Sons, Inc.

Baltadjiev N.D., (2012). An Investigation of Real Gas Effects in Supercritical $\mathrm{CO}_{2}$ Centrifugal Compressors. Masters of Science, Massachusetts Institute of Technology. Boston, USA.

Baltadjiev N.D., Lettieri C, Spakovszky Z., (2014). An Investigation of Real Gas Effects in Supercritical $\mathrm{CO}_{2}$ Centrifugal Compressors. Proceedings of the ASME Turbo Expo, Paper No. GT2014-26180. Düsseldorf, Germany.

Bauer M.L., Vijaykumar R., Lausten M., and Stekli J.. (2016). Pathways to cost competitive concentrated solar power incorporating supercritical carbon dioxide power cycles. 
Benmeddour A., (2017). MECH 4304 Computational Fluid Dynamics. Carleton University, Department of Mechanical and Aerospace Engineering. Ottawa, ON, Canada.

Burk, R., (1991). Supercritical Fluid Extraction of Trace Organics from Solid Matrices. $\mathrm{Ph}, \mathrm{D}$. dissertation, Department of Chemistry, Carleton University, Ottawa, ON, Canada.

Brun K., Friedman P., Dennnis R., (2017). Fundamentals and Applications of Supercritical Carbon Dioxide $\left(\mathrm{sCO}_{2}\right)$ Based Power Cycles. Elsevier Ltd, $1^{\text {st }}$ ed, Woohead Publishing, UK.

Crespi F., Gavagnin G., Sanchez D., Martinez S.G., (2017). Supercritical Carbon Dioxide Cycles for Power Generation: A Review. Journal of Applied Energy. ElSevier. Vol. 195. June. Pg. 152-183.

Crespi F., Gavagnin G., Sanchez D., Martinez S.G., (2017). Analysis of the Thermodynamic Potential of Supercritical Carbon Dioxide Cycles: A Systematic Approach. Proceedings of the ASME Turbo Expo, Paper No. GT2017-64418. Charlotte NC, USA.

Dixon S.L., and Hall C.A., (2014). "Fluid Mechanics and Thermodynamics of Turbomachinery", $7^{\text {th }}$ ed. El Sevier, United States. ISBN: 978-0-12-415954-9

Dincer I., and Zamfirescu C., (2014), “Advanced Power Generation Systems”, $1^{\text {st }}$ ed. El Sevier, Web ISBN-13: 978-0-12-383861-2.

Dostal V., (2004). A Supercritical Carbon Dioxide Cycle for Next Generation Nuclear Reactors, Ph, D. dissertation, Advanced Nuclear Power Technology Program, MIT, MA, USA, 2004.

Drew R., (2012). Special Report - Supercritical CO2 The next big step?, The Global Journal of Energy Equipment - Turbomachinery International, Vol 53, Numb 5, Sep/Oct 2012, pp 22-28.

Eckardt D,. (1980), Flow Field Analysis or Radial and Backswept Centrifugal Compressor Impellers. Part I: Flow Measurements Using A Laser Velocimeter. Conference proceeding, New Orleans, USA.

Fuller R.F., Jason P., and Jeff N., (2012). Turbomachinery for Supercritical CO2 Power Cycles. Proceeding of the ASME Turbo Expo. Paper No. GT2012-68735. Copenhagen, Denmark.

Japikse D., and Baines N.C., (1997) Introduction to Turbomachinery. Oxford University Press. New York. 
Kibsey, M., (2016). Internal Design Report of a Centrifugal Compressor and Radial Turbine - Carleton University, Department of Mechanical and Aerospace Engineering. Ottawa, ON, Canada.

Market and Markets (2016), Waste Heat Recovery System Market by Application (Preheating and Steam \& Electricity Generation), End-Use Industry (Petroleum Refining, Metal Production, Cement, Chemical, Paper \& Pulp, and Textile) - Global Trends \& Forecasts to 2021. September 2016, Report Code: CH 2382. US.

Musgrove G., Wilkes J., (2016), "Tutorial Fundamentals of Supercritical CO2", Supercritical CO2 Power Cycle Symposium 2016, San Antonio Texas, March 29-31.

Monje B., Sanchez D., Savill M., Pilidis P., and Sanchez T., (2014). A Design Strategy for Supercritical CO2 Compressors. Proceedings of the ASME Turbo Expo, Paper No. GT2014-25151. Düsseldorf, Germany.

Monje B., (2014) Design of Supercritical Carbon Dioxide Centrifugal Compressors. PhD Thesis. Universidad de Sevilla, Spain

Moraga F., Hofer D., Saxena S., Mallina R., (2017), GE Global Research Center. "Numerical Approach for Real Gas Simulations - Part I: Tabular Fluid Properties for Real Gas Analysis". Proceedings of the ASME Turbo Expo, Paper No. GT2017-63149. Charlotte NC, USA

Moran M.J., Shapiro H.N., (2011). Fundamentals of Engineering Thermodynamics, $7^{\text {th }}$, United States of America, John Wiley \& Sons, Inc. ISBN- 13 978-0470-49590-2

Pham H.S. et al., (2016). An Approach for Establishing the Performance Maps of the ScCO2 Compressor: Development and Quantification by Means of CFD Simulation. International Journal of Heat and Fluid Flow.

REFPROP V9.1 (2015). NIST Standard Reference Database 23: Reference Fluid Thermodynamic and Transport Properties-REFPROP.

Rochau G.R., Drennen T., Pasch J., Fleming D., Carlson M., Dawson L., Kruizenga A., (2016). Commercialization of SCO2 Brayton Power Cycles, Supercritical CO2 Power Cycle Symposium 2016, San Antonio Texas, March 29-31.

SANDIA Report, (2013). Fleming D.D., Conboy T.M., Pasch J.J., Rochau G.A., Fuller R.L., Holschuh T.V., Wright S.A., Scaling Considerations for a Multi-Megawatt Class Supercritical CO2 Brayton Cycle and Commercialization. SAND2013-9106, Albuquerque, New Mexico, 2013

Saravanamuttoo, H.I.H., Rogers G.F.C., Cohen H., Straznicky P.V., (2009). Gas Turbine Theory, $6^{\text {th }}$ ed., Pearson Education Limited, UK. 
Saxena S., Mallina R., Moraga F., Hofer D., (2017), GE Global Research Center. "Numerical Approach for Real Gas Simulations - Part II: Flow Simulation for Supercritical CO2 Centrifugal Compressor". Proceedings of the ASME Turbo Expo, Paper No. GT2017-63149. Charlotte NC, USA

Sjolander S.A (2017) "MECH 4305 Fluid Machinery Supplementary Notes”, Chapter 2. Carleton University, Department of Mechanical and Aerospace Engineering. Ottawa, ON, Canada.

Sjolander S.A (2015) MECH 4305 Fluid Machinery Supplementary Notes (Fall 2015). Carleton University, Department of Mechanical and Aerospace Engineering. Ottawa, ON, Canada.

Span R. and Wagner, (1996). A New Equation of State for Carbon Dioxide Covering the Fluid Region from the Triple-Point Temperature to $1000 \mathrm{~K}$ at Pressures up to $800 \mathrm{MPa}$. Journal Phys. Chem. Ref Data. 25(6): 1509-1596.

Suppes G.J., and Storvick T.S., (2015). Sustainable Power Technologies and Infrastructure: Energy Sustainability and Prosperity in a Time of Climate Change, $1^{\text {st }}$ ed, Science and Technologies Books inc, 2015. Pp 414. E- Book ISBN: 9780128039281.

U.S Department of Energy, (2008). Industrial Technologies Program "Waste Heat Recovery: Technology and Opportunity in US Industry”. USA.

White Martin, (2015). The design and analysis of radial inflow turbines implemented within low temperature organic Rankine cycles. $\mathrm{Ph}, \mathrm{D}$. dissertation, School of Mathematics, Computer Science \& Engineering Department of Mechanical Engineering \& Aeronautics, University of London, London, 2015.

White Frank M., (2010). Fluid Mechanics. McGraw-Hill Education, $7^{\text {th }}$ ed. US. ISBN10: 0077422414

Wright Steven A., Vernon Milton E., Radel Ross F., Fuller Robert L., (2009). Supercritical $\mathrm{CO}_{2}$ Main Compressor Performance Measurements". Sandia National Laboratories. SAND2009-0980C. Albuquerque, New Mexico USA.

Wright S.A., Radel R.F., Vernon M.E., Rochau G.E., Pickard Paul S., (2010). Operation and Analysis of Supercritical $\mathrm{CO}_{2}$ Brayton Cycle. Sandia National Laboratories. Sandia Report SAND2010-0171. Albuquerque, New Mexico USA.

Wright S.A., Davidson C.S., Scammell W.O., (2016). Thermo-Economic Analysis of Four S-CO2 Waste Heat Recovery Power Systems. Supercritical CO2 Power Cycle Symposium 2016, San Antonio Texas, March 29-31. 


\section{Appendix A: List of Identified Waste Heat Sources}

Table 7-2 presents a list of some of the identified waste heat sources and indicates the operating temperatures. Notice that the temperature range of the heat sources lie within the temperature operating rage of $\mathrm{S}_{-} \mathrm{CO}_{2}$ Brayton cycles presented in 2.3.1 Expected Efficiency and Temperature Operating Range.

Table A-1: List of Recoverable Waste Heat Sources of High Temperature (Dincer and Zamfirescu, 2014).

\begin{tabular}{|l|c|}
\hline Thermal Source & Temperature $\left({ }^{\circ} \mathrm{C}\right)$ \\
\hline Steam power plants & $230-250$ \\
\hline Gas turbine power plants & $600-800$ \\
\hline Heating furnace exhaust & $175-230$ \\
\hline Automobile engine exhaust & $400-700$ \\
\hline Nitrogenous fertilizers plants & $195-230$ \\
\hline Pulp mills & $140-200$ \\
\hline Paper mills & $140-200$ \\
\hline Paperboard mills & $140-200$ \\
\hline Alkalines and chlorines production & $170-220$ \\
\hline Industrial inorganic chemical production & $120-200$ \\
\hline Industrial organic chemicals & $120-300$ \\
\hline Petroleum crudes and intermediate refining & $150-200$ \\
\hline Glass factories & $400-500$ \\
\hline Cement production & $200-300$ \\
\hline Blast furnaces & $500-700$ \\
\hline Iron foundries & $425-650$ \\
\hline Drying and baking ovens & $93-230$ \\
\hline Aluminum production & $650-760$ \\
\hline Copper production & $760-815$ \\
\hline
\end{tabular}




\section{Appendix B: Initial Sizing Using the Flow $(\phi)$ and Work $(\psi)$}

\section{Coefficient}

Aungier (2000) presented a set of charts which correlate the Work $\left(\psi_{1}\right)$ coefficient versus Flow $\left(\phi_{1}\right)$ coefficient. These charts can be used to guide the initial sizing of the compressor. The charts are divided by their configurations; shrouded or unshrouded impellers and vaneless or vanned diffusers. The charts were developed from empirical data and correlations corresponding to well-designed industrial centrifugal compressors. It is important to understand that the charts presented by Aungier are valid for compressors with a pressure ratio up to approximately 3.5 . These charts are meant to provide initial sizing of components that provide a stable operating range (Aungier, 2000).

The experimental results used by Aungier corresponded to a Flow $\left(\phi_{1}\right)$ coefficient of 0.009 to 0.125 , however, Aungier developed correlations to extend the Flow $\left(\phi_{1}\right)$ coefficient to 0.003 and up to 0.2 (Aungier, 2000). The charts use polytropic $\left(\eta_{p}\right)$ efficiencies to reduce the dependence on pressure ratio. Figure A-1 illustrates the chart corresponding to an unshrouded impeller.

Aungier defines the non-dimensional parameters of Flow $\left(\phi_{1}\right)$ and Work $\left(\psi_{1}\right)$ coefficient as shown in Equation A.1 and A.2

$$
\begin{aligned}
& \phi_{1}=\frac{\dot{m}}{\rho_{o_{1}} \pi r_{2}^{2} U_{2}} \\
& \psi_{1}=\frac{\Delta h_{o, r e f}}{U_{2}^{2}}
\end{aligned}
$$

Where:

$\dot{m}-$ Mass Flow, $\rho_{1}$-Impeller Inlet Density, $r$-Impeller Outlet Radius 
$U_{2}-$ Blade Speed and $\Delta h_{o, r e f}-$ Total Isentropic Enthalpy Change

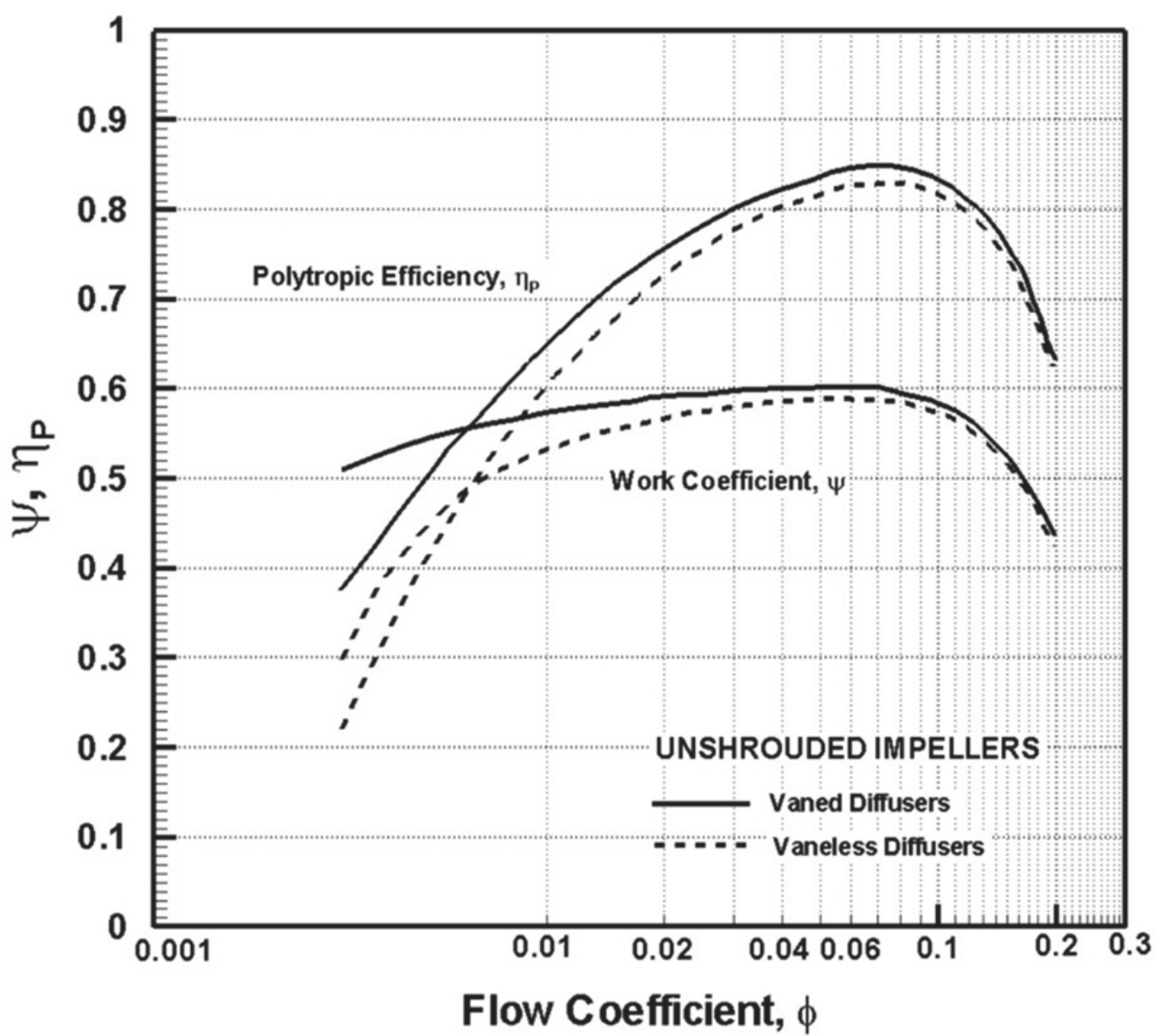

Figure B-1: Work Coefficient vs. Flow Coefficient covered impeller design targets (Sjolander, 2015).

Following Aungier's methodology, the designer selects a Flow Coefficient $\left(\phi_{1}\right)$ and reads the corresponding work coefficient $\left(\psi_{1}\right)$. Typically, the selected value of $\phi_{1}$ corresponds to the highest efficiency $\left(\eta_{p}\right)$, unless, the designer has the rotational speed (N) set as a constraint.

Equation A.2 can be rearranged to solve for the blade speed $\left(\mathrm{U}_{2}\right)$, and Equation A.1 can be rearranged to determine the tip radius $\left(\mathrm{r}_{2}\right)$. Then the rotational speed $(\mathrm{N})$ can be calculated as shown by Equation A.3.

$$
N=\frac{60 * U_{e}}{2 \pi r_{e}}
$$

